Ni-catalyzed cycloisomerization between 3-phenoxy acrylic acid derivatives and alkynes via intramolecular cleavage and formation of C-O bond to give 2,3disubstituted benzofurans

Shohei Ohno, ${ }^{1}$ Jiawei Qiu, ${ }^{1}$ Ray Miyazaki, ${ }^{2}$ Hiroshi Aoyama, ${ }^{1}$ Kenichi Murai, ${ }^{1}$ Jun-ya Hasegawa, ${ }^{2}$ and Mitsuhiro Arisawa*

${ }^{1}$ Graduate School of Pharmaceutical Sciences, Osaka University, Yamada-oka 1-6, Suita, Osaka, 565-0871 JAPAN

${ }^{2}$ Institute for Catalysis, Hokkaido University, Kita 21 Nishi 10, Kita-ku, Sapporo, Hokkaido, 001-0021 JAPN

*Corresponding Author. Phone: +81-6-6879-8226

E-mail: arisaw@phs.osaka-u.ac.jp

Contents

NMR spectra 
single _pulse

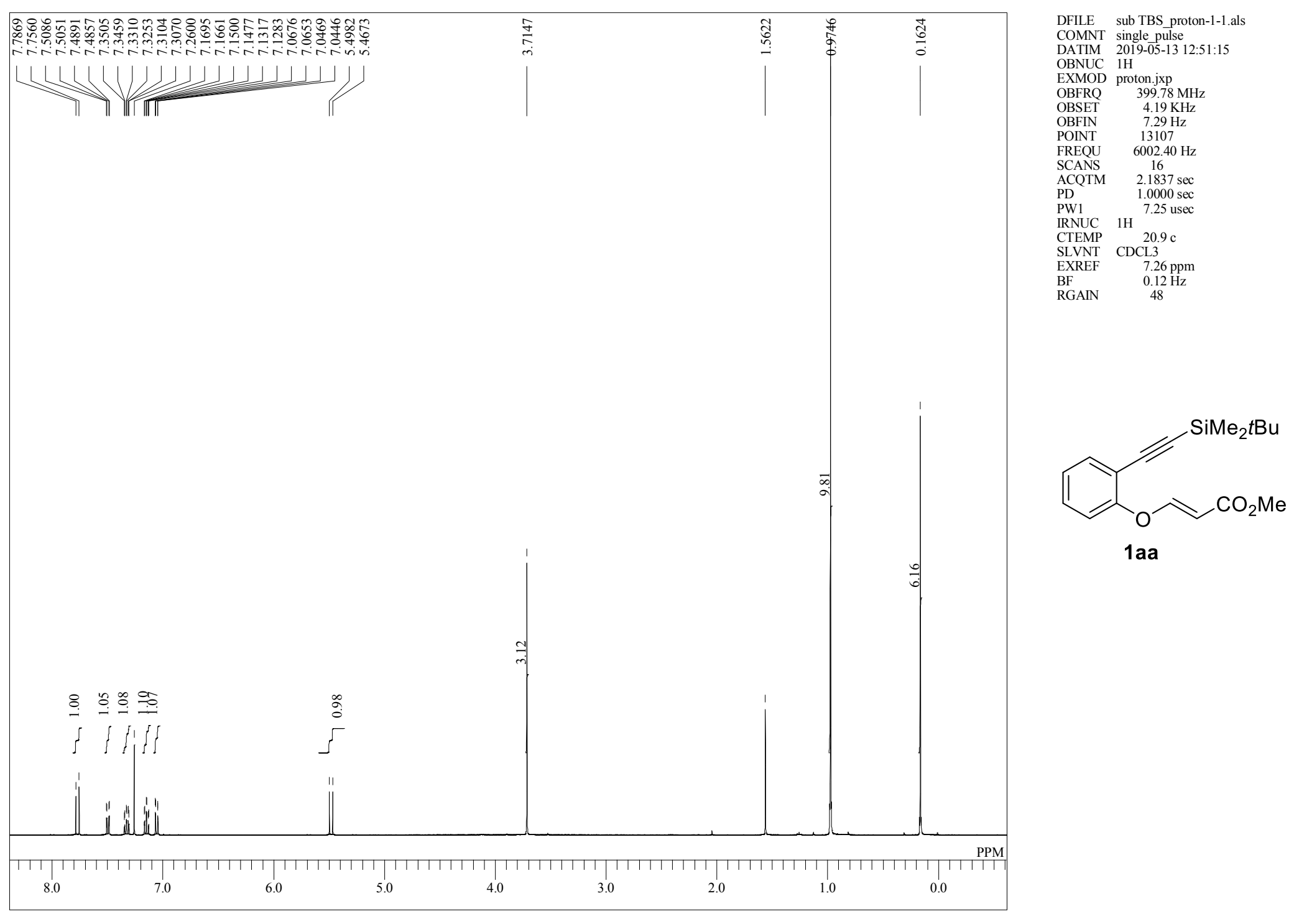

$\mathrm{S} 2-2$ 
single pulse decoupled gated NOE

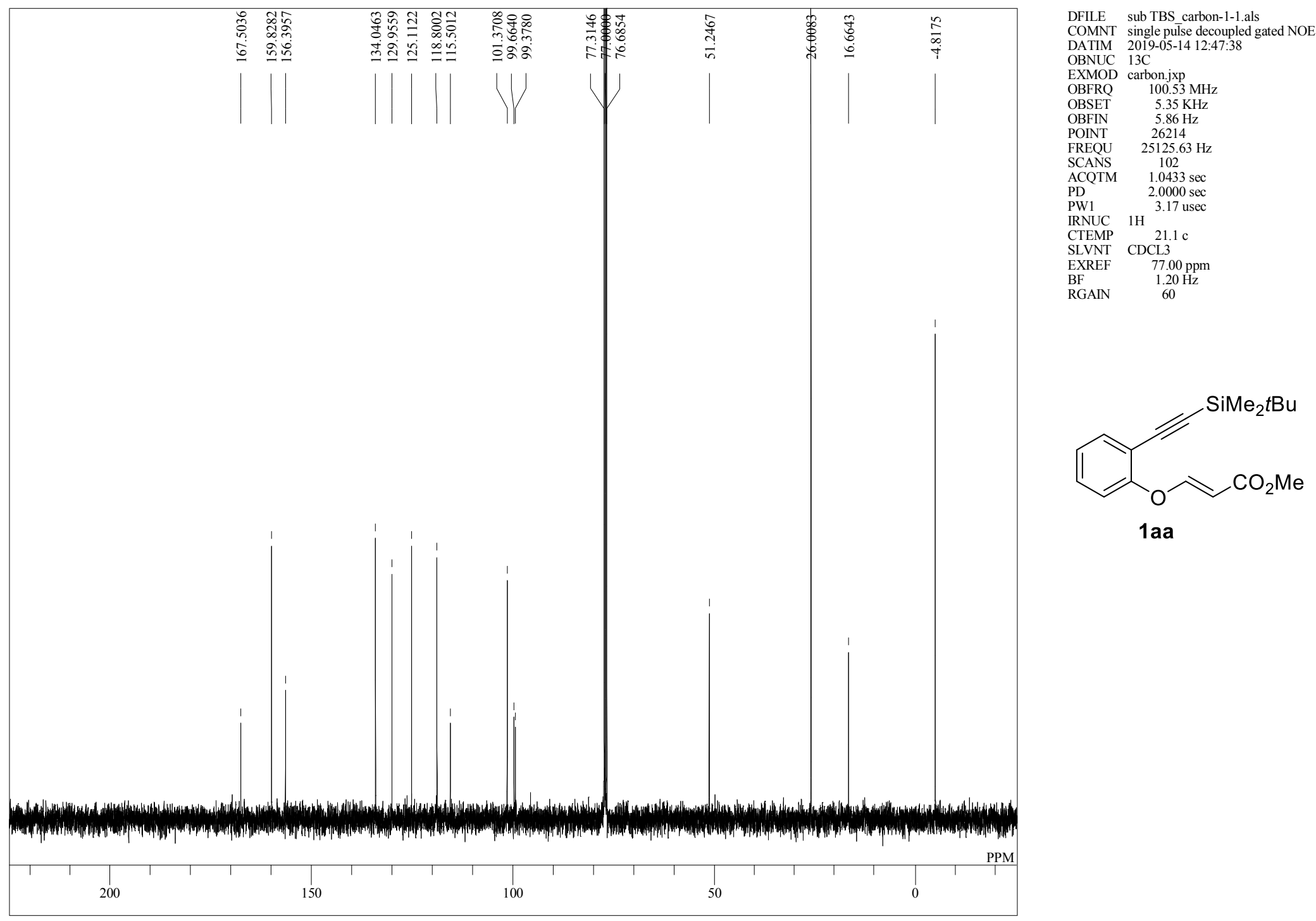

S2- 3 
single pulse

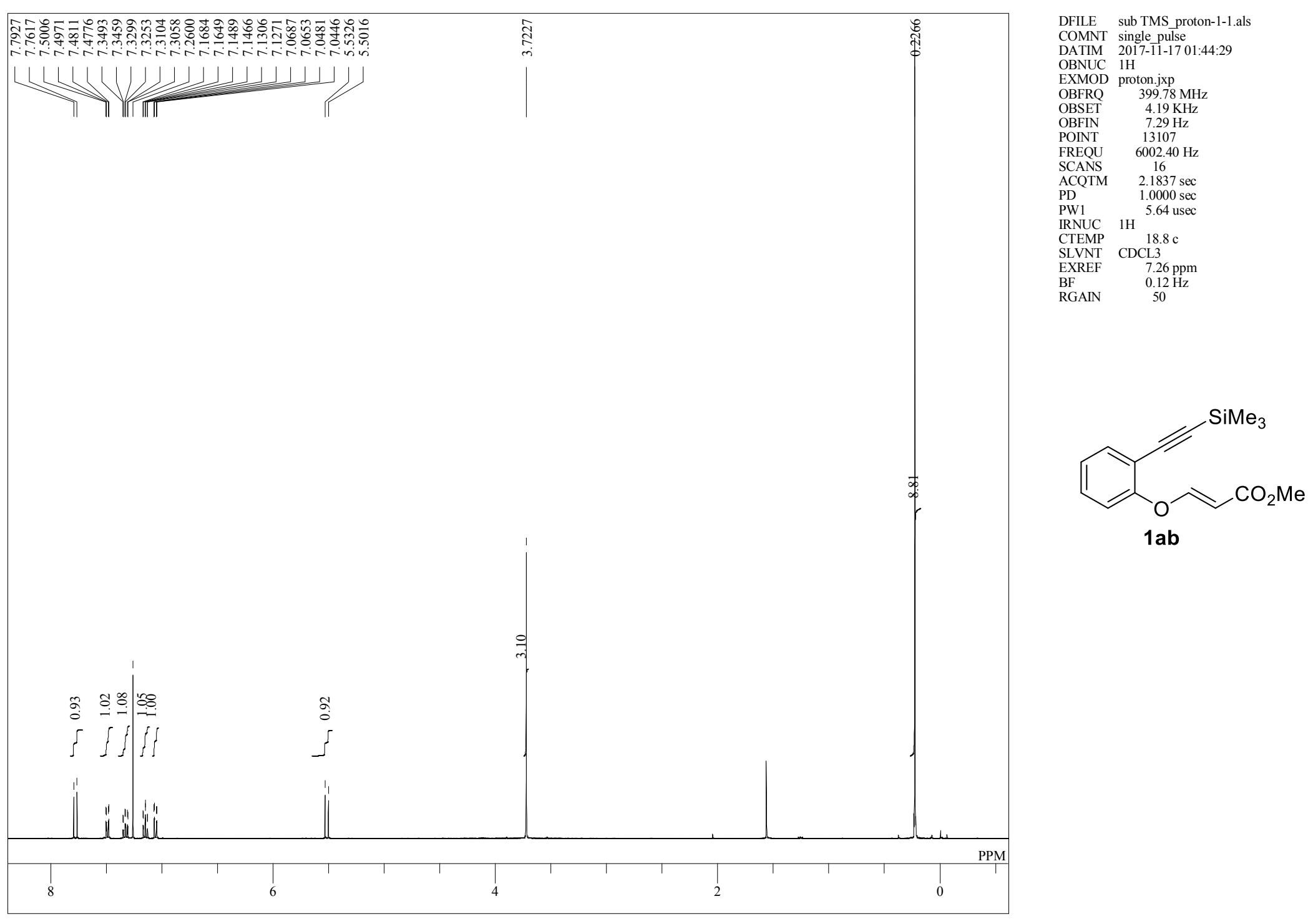

$\mathrm{S} 2-4$ 
single pulse decoupled gated NOE

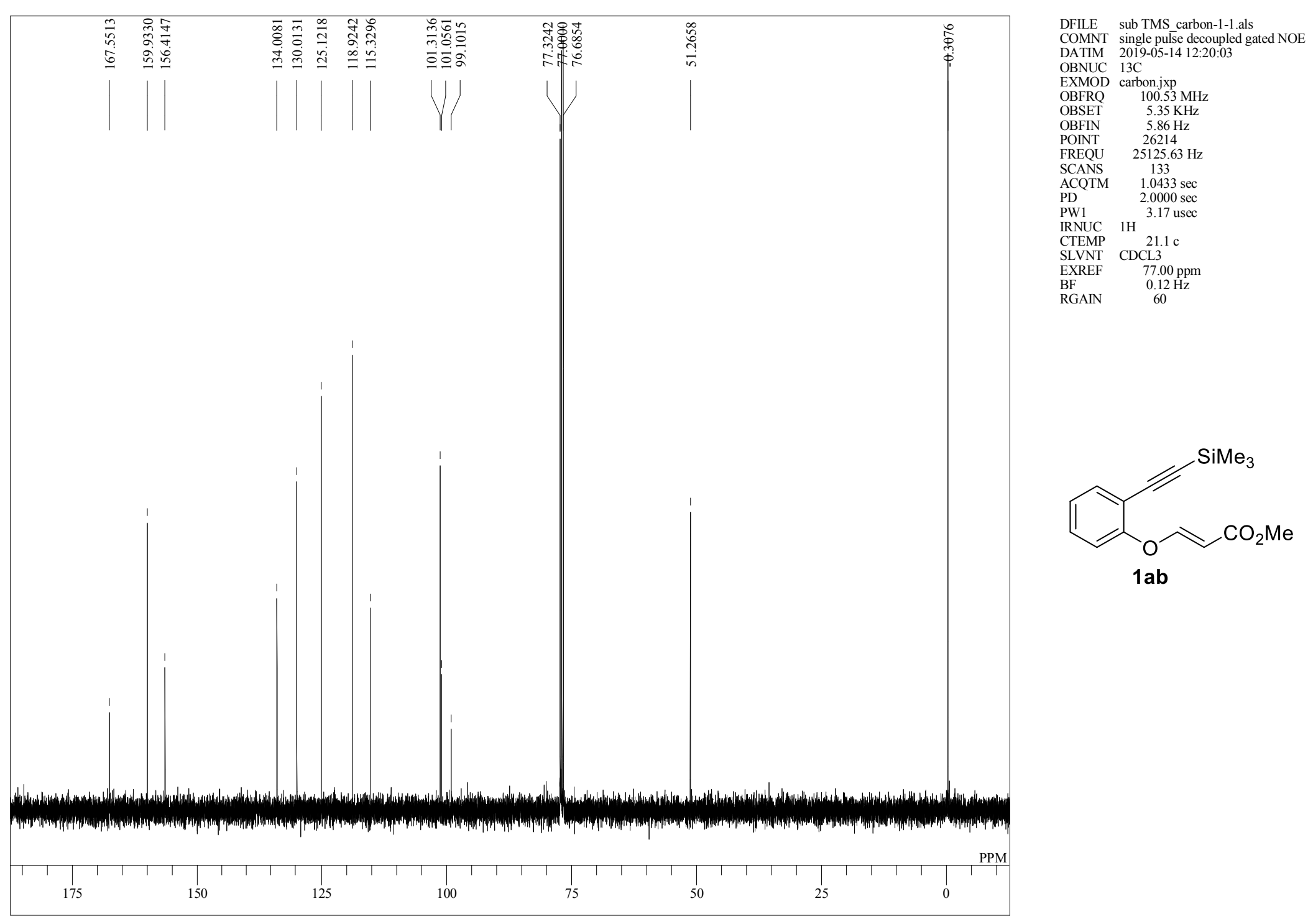

$\mathrm{S} 2-5$ 
single pulse

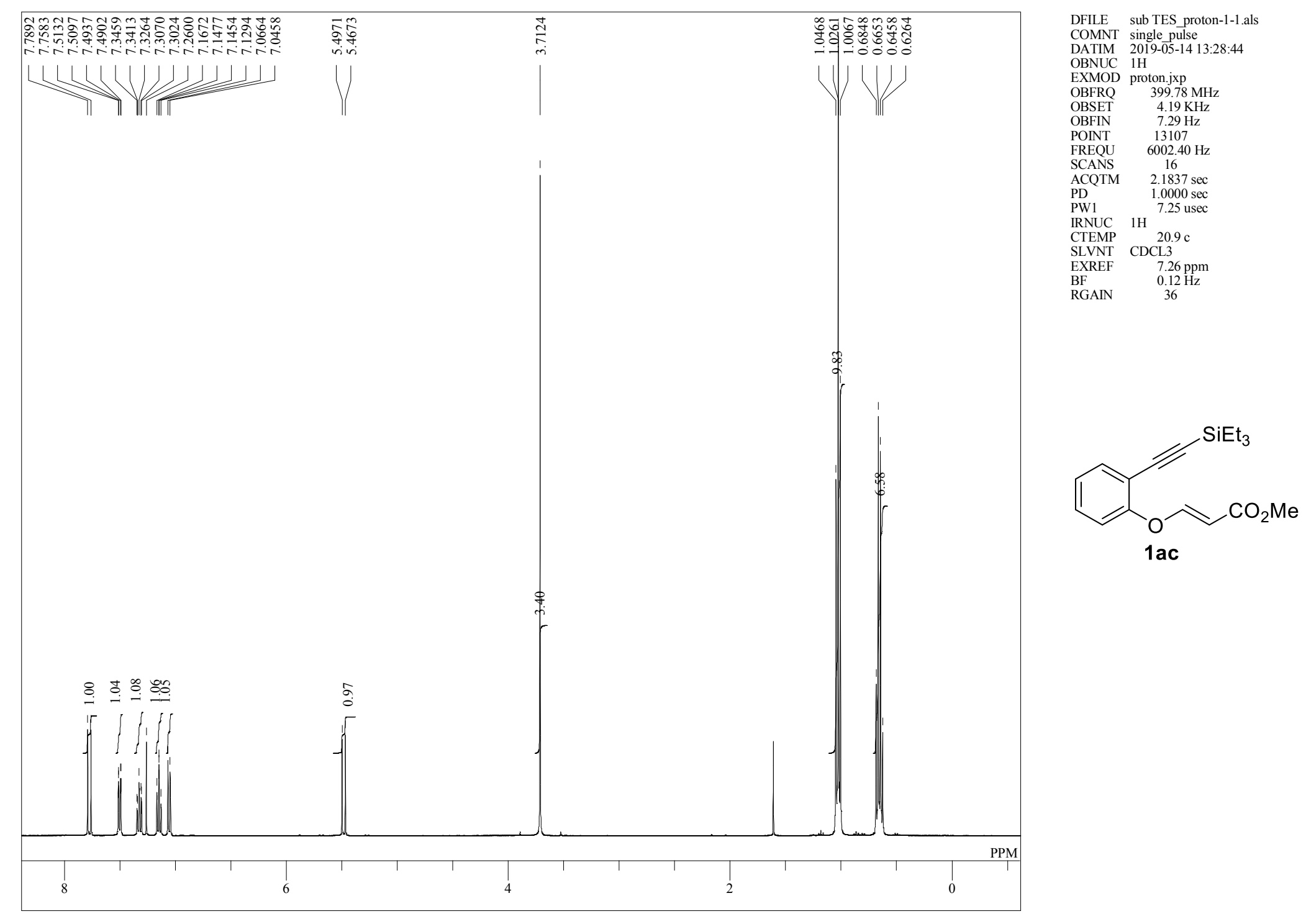

S2- 6 
single pulse decoupled gated NOE

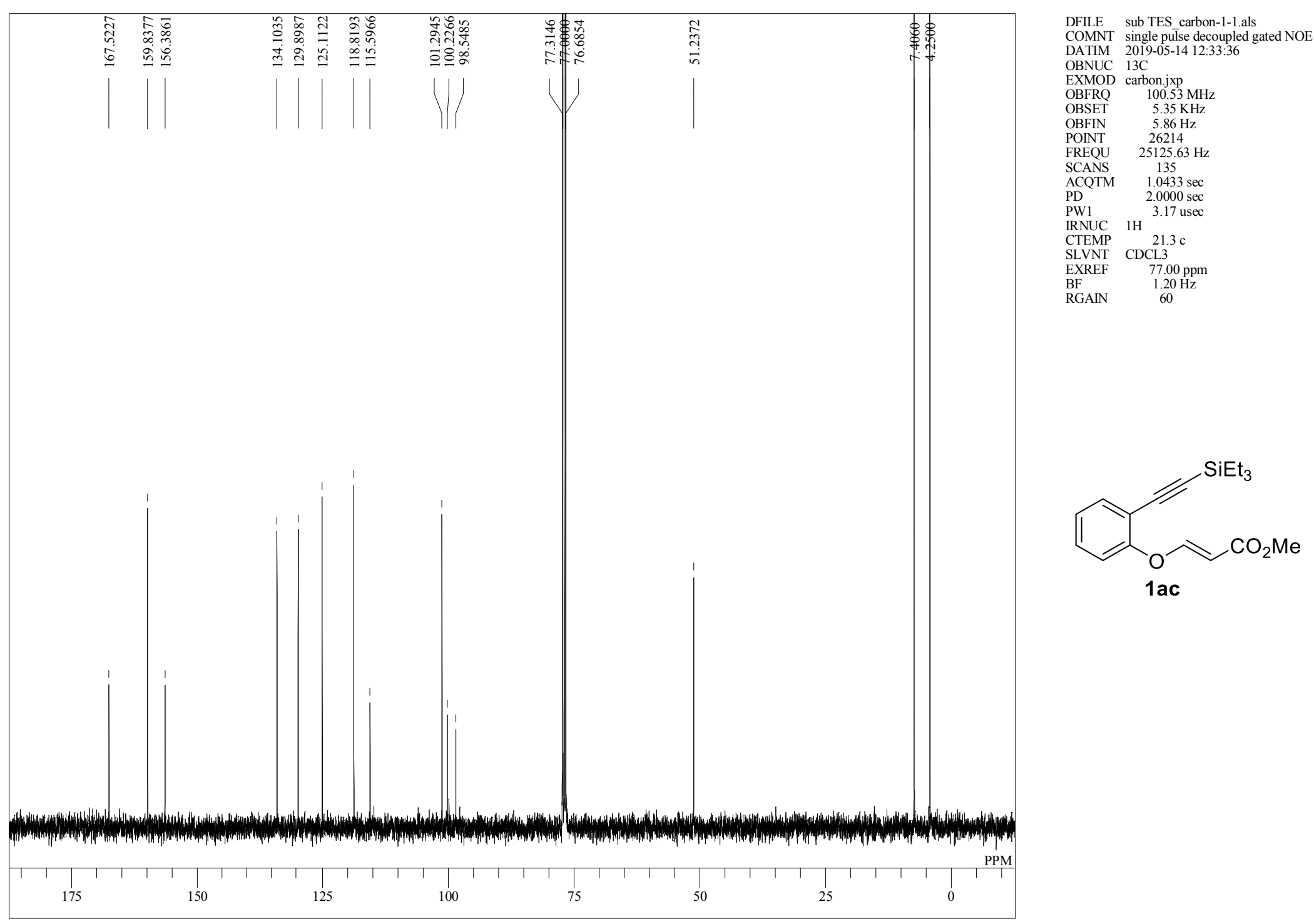

$\mathrm{S} 2-7$ 
single pulse

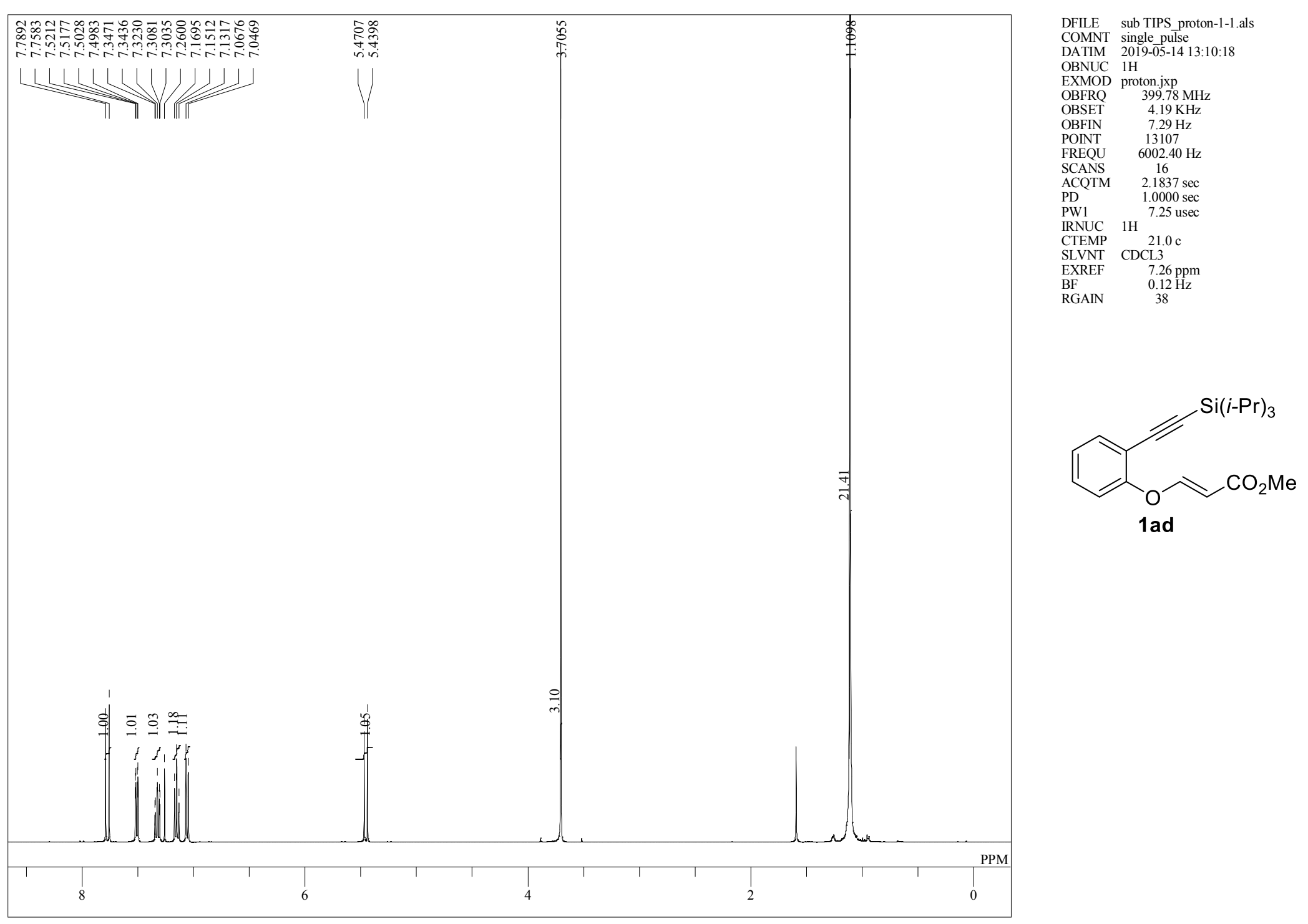

S2- 8 
single pulse decoupled gated NOE

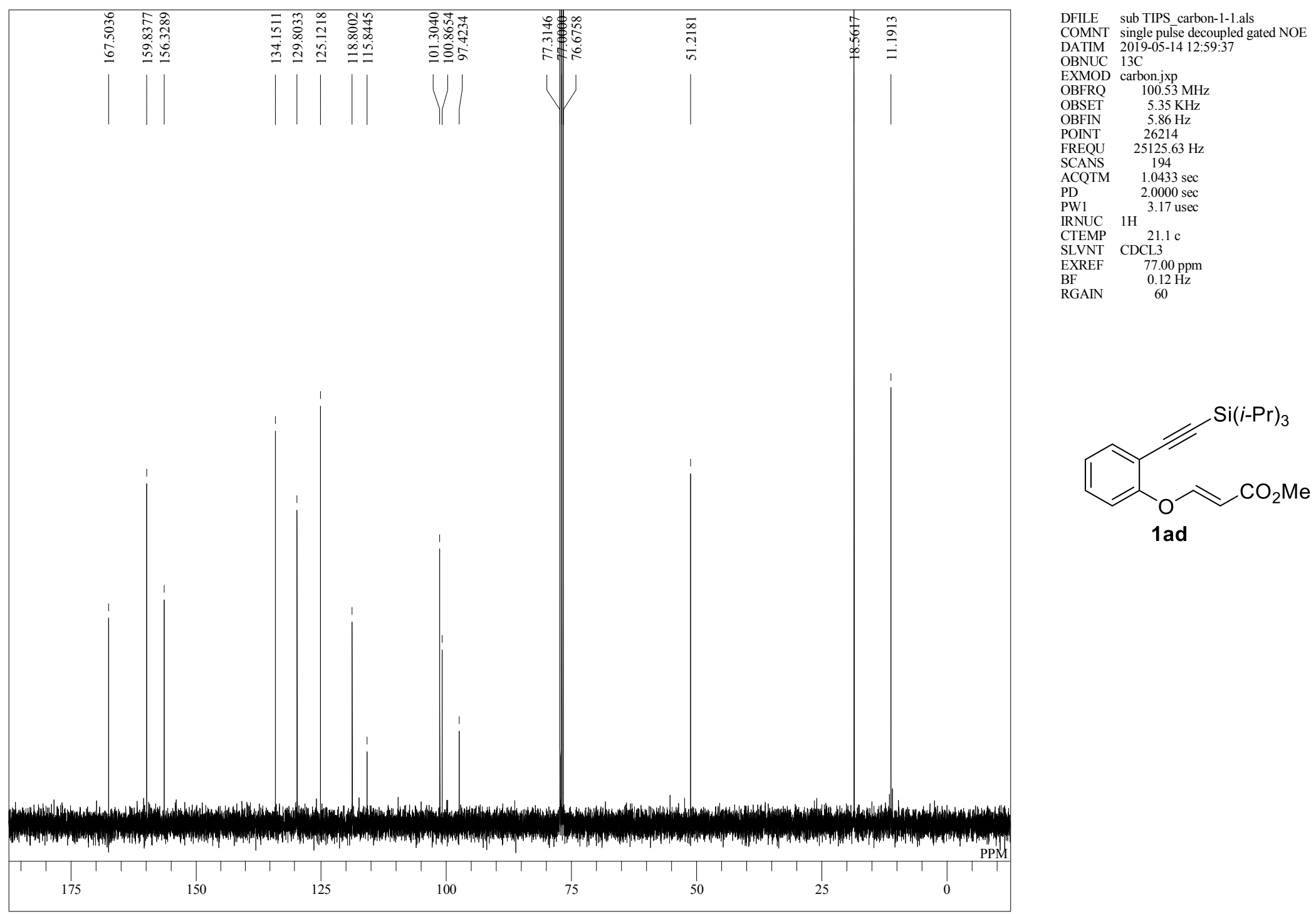

S2- 9 
single _pulse

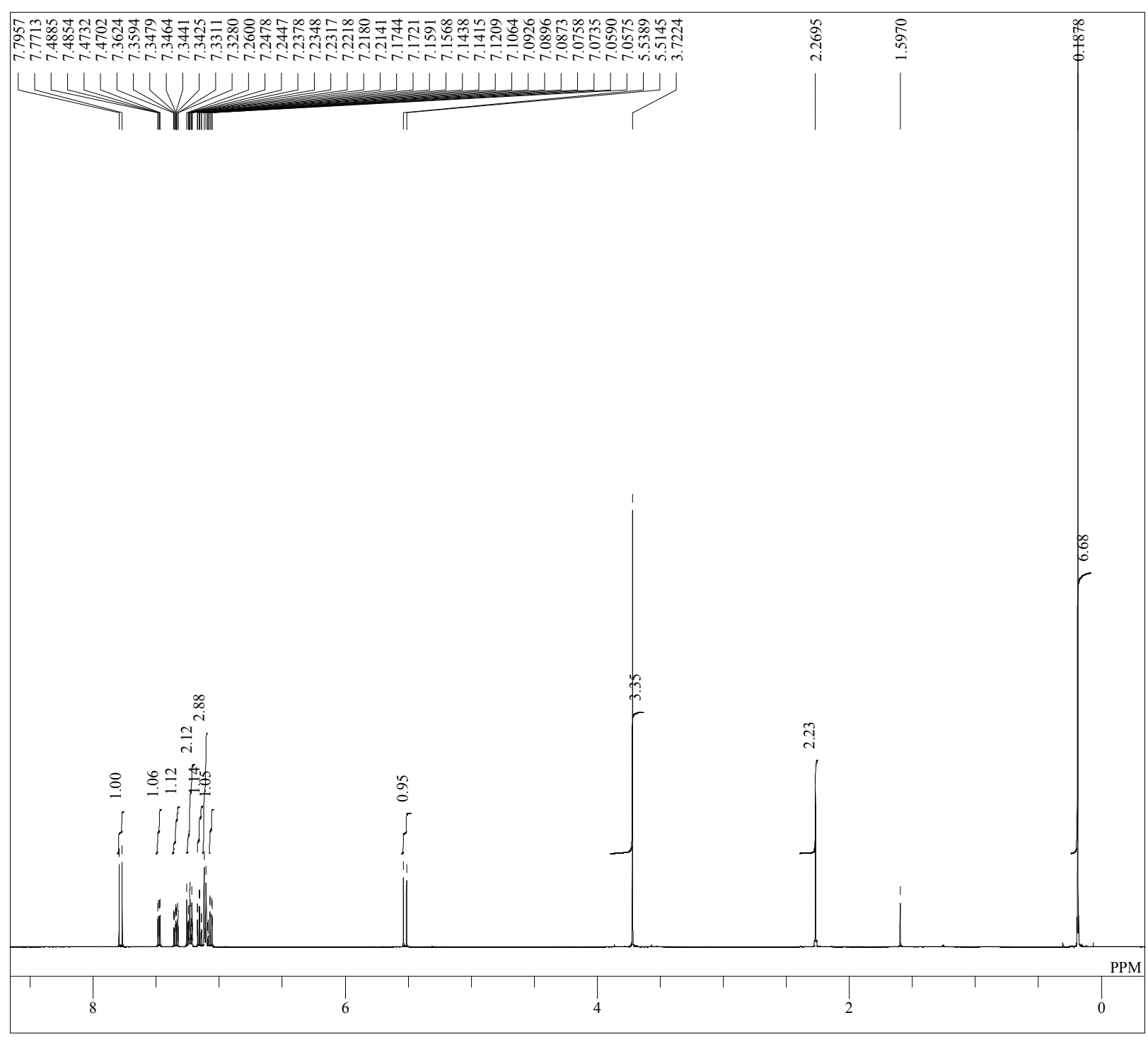

DFILE sub Sime2Bn_proton-1-1.als

$\begin{array}{ll}\text { COMNT } & \text { single-pulse } \\ \text { DATIM } & 2019-05-14 \\ \text { 14:54:43 }\end{array}$

OBNUC $1 \mathrm{H}$

$\begin{array}{ll}\text { EXMOD } & \text { proton.jxp } \\ \text { OBFRQ } & 500.16 \mathrm{MHz}\end{array}$

OBSET $\quad 2.41 \mathrm{KHz}$

POINT 26214

FREQU $\quad 10020.04 \mathrm{H} z$

$\begin{array}{ll}\text { SCANS } & 16 \\ \text { ACOTM } & 2.6162 \mathrm{sec}\end{array}$

$\begin{array}{ll}\mathrm{ACQTM} & 2.6162 \mathrm{sec} \\ \mathrm{PD} & 20000 \mathrm{sec}\end{array}$

PW1 6.50 usec

IRNUC $1 \mathrm{H}$

$\begin{array}{ll}\text { CTEMP } & 19.9 \mathrm{c} \\ \text { SLVNT } & \text { CDCL3 }\end{array}$

$\begin{array}{ll}\text { EXREF } & 7.26 \mathrm{ppm} \\ \mathrm{BF} & 0.12 \mathrm{~Hz}\end{array}$

RGAIN $\quad .12 \mathrm{~Hz}$

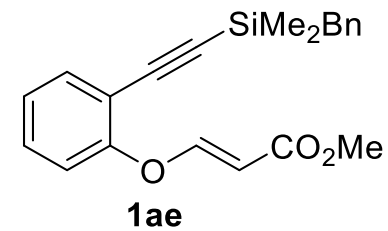

S2 - 10 
single pulse decoupled gated NOE

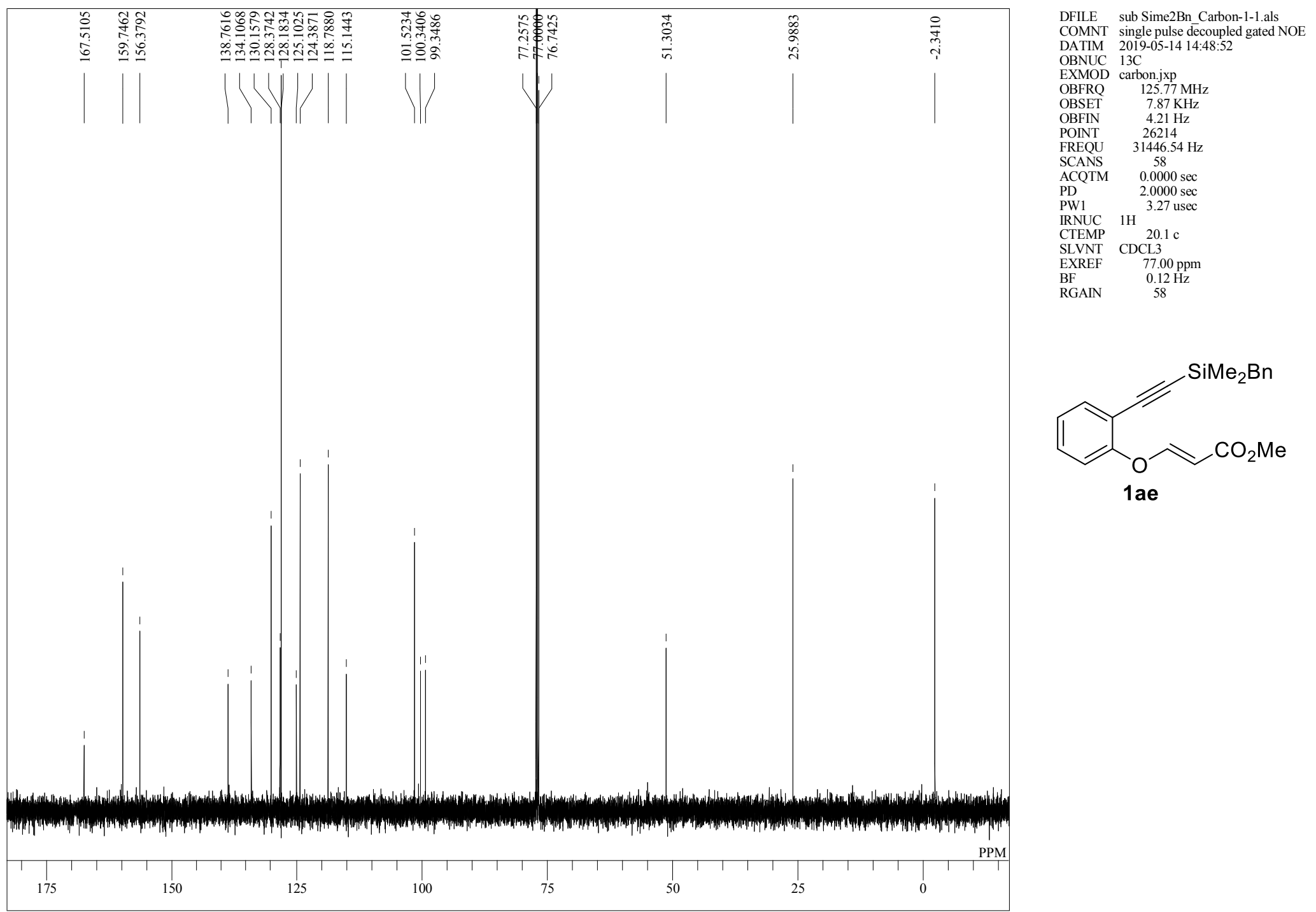


single pulse

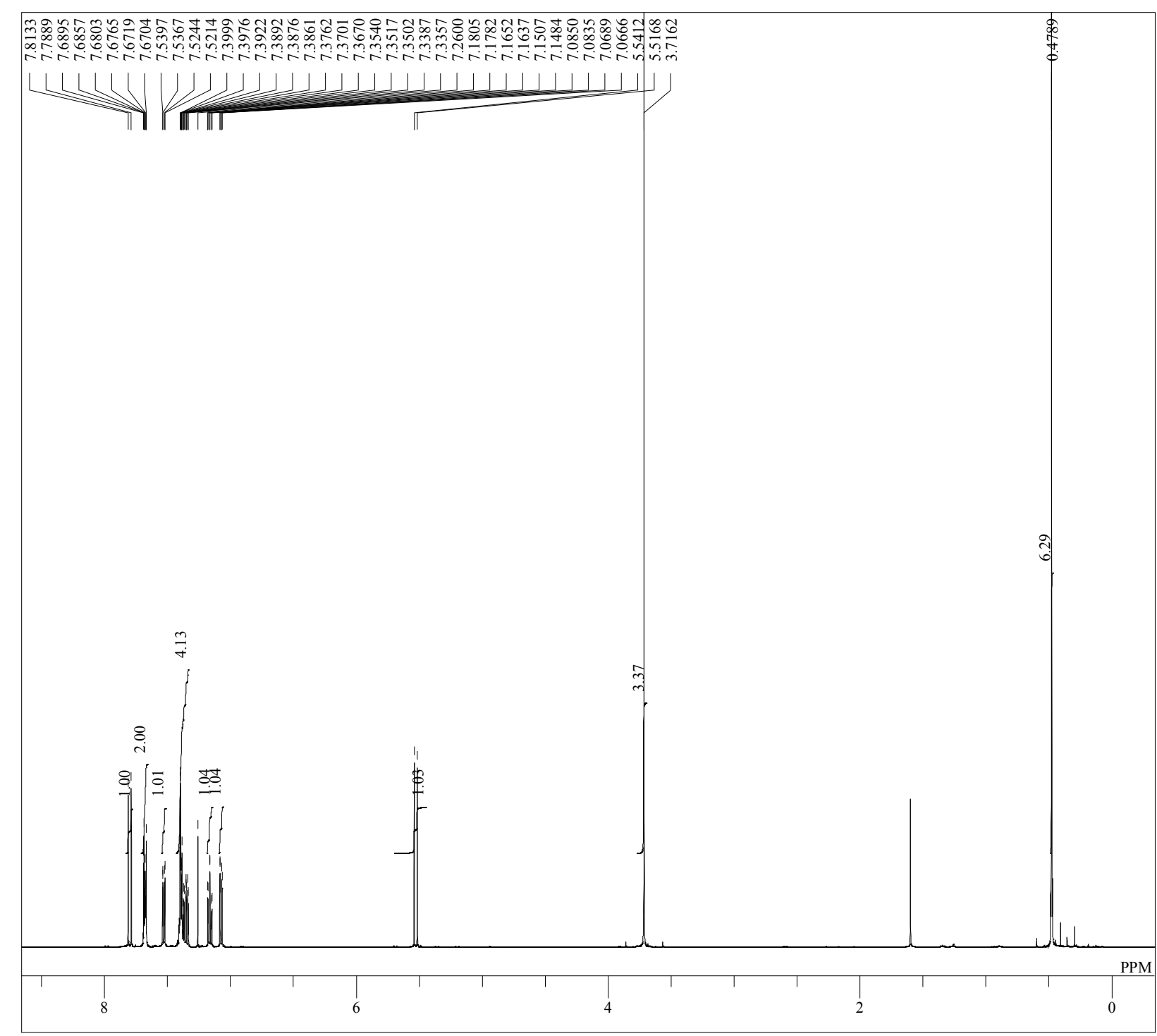

DFILE sub Sime2Ph proton-1-1.als

DATIM 2019-05-14 14:25:03

OBNUC $1 \mathrm{H}$

$\begin{array}{ll}\text { EXMOD } & \text { proton.jxp } \\ \text { OBFRQ } & 500.16 \mathrm{MHz}\end{array}$

OBSET $\quad 2.41 \mathrm{KHz}$

POINT 26214

FREQU $10020.04 \mathrm{~Hz}$

$\begin{array}{ll}16 \\ \text { ACATM } & 2.6162 \mathrm{sec}\end{array}$

$\begin{array}{ll}\mathrm{PD} & 2.6162 \mathrm{sec} \\ & 0.500 \mathrm{sec}\end{array}$

PW1 $\quad 6.50$ usec

IRNUC $1 \mathrm{H} \quad 19.9 \mathrm{c}$

$\begin{array}{ll}\text { CTEMP } & 19.9 \mathrm{c} \\ \text { SLVNT } & \text { CDCL } 3\end{array}$

$\begin{array}{ll}\text { EXREF } & 7.26 \mathrm{ppm} \\ \mathrm{BF} & 0.12 \mathrm{~Hz}\end{array}$

RGAIN 40

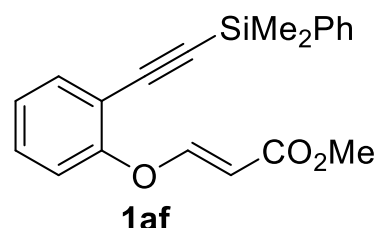

$\mathrm{S} 2-12$ 
single pulse decoupled gated NOE

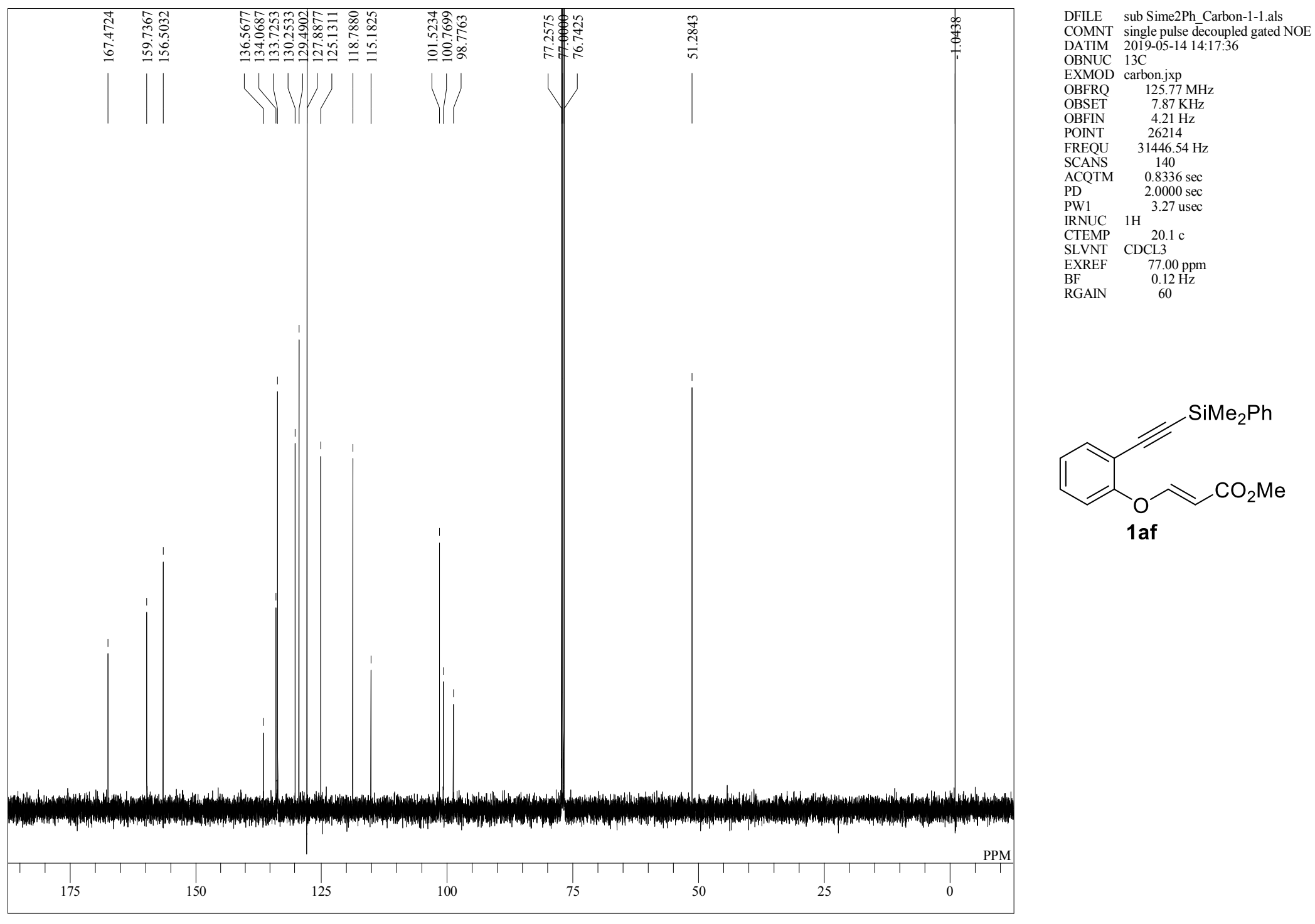


single pulse

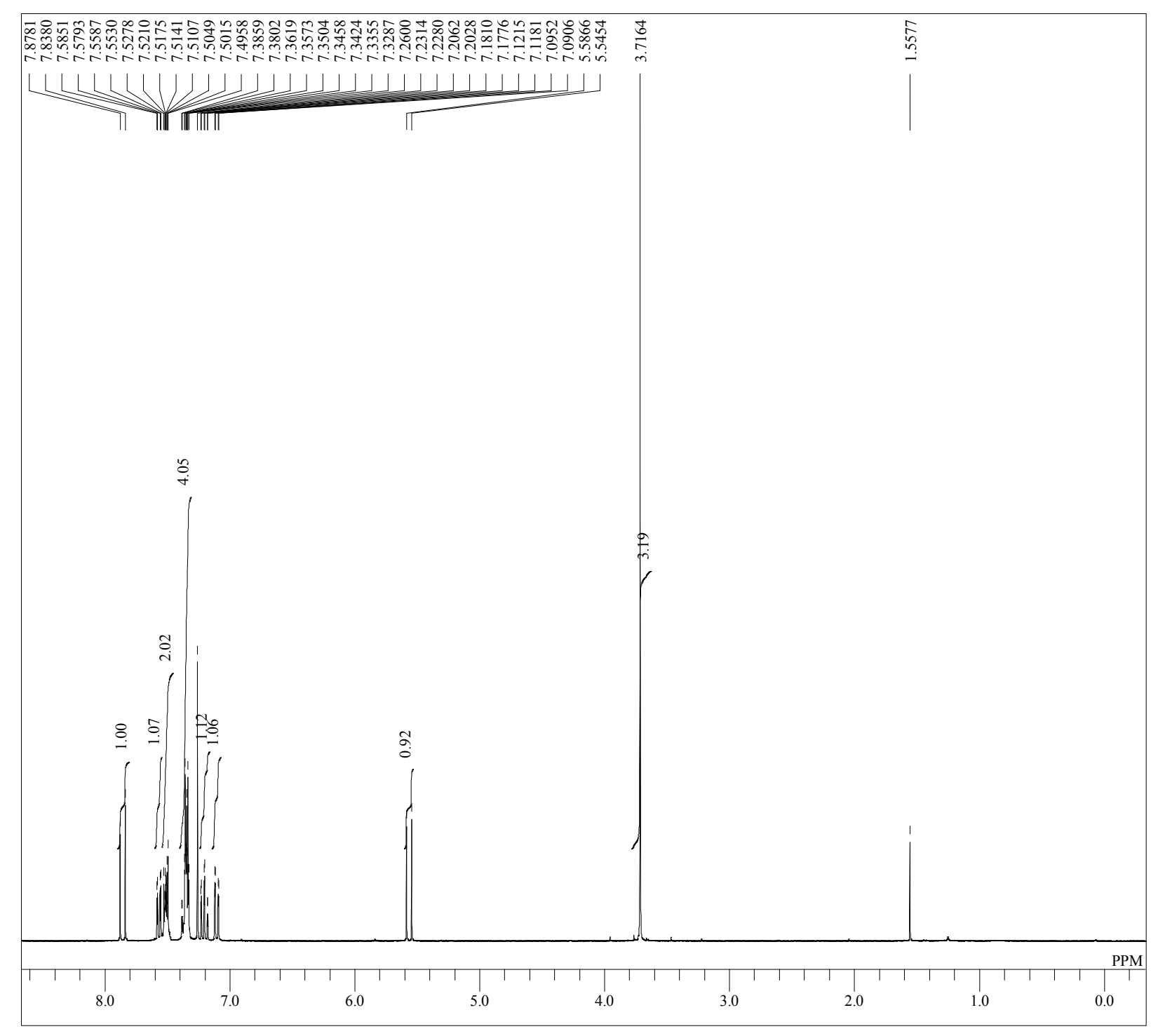

DFILE sub Ph proton-1-1.als

COMNT single pulse

OBNUC $1 \mathrm{H}$

EXMOD pro

$\begin{array}{ll}\text { OBFRQ } & 300.53 \mathrm{MHz} \\ \text { OBSET } & 1.15 \mathrm{KHz}\end{array}$

$\begin{array}{ll}\text { OBSET } & 1.15 \mathrm{KHZ} \\ \text { OBFIN } & 857 \mathrm{~Hz}\end{array}$

POINT $\quad 13107$

FREQU $\quad 4508.57 \mathrm{~Hz}$

SCANS $16 \quad 16$

$\begin{array}{ll}\text { ACQTM } & 2.9072 \mathrm{sec} \\ \mathrm{PD} & 1.0000 \mathrm{sec}\end{array}$

PW1 5.50 usec

IRNUC $1 \mathrm{H} 21.3 \mathrm{c}$

$\begin{array}{lr}\text { CTEMP } & 21.3 \\ \text { SLVNT } & \text { CDCL3 }\end{array}$

$\begin{array}{ll}\text { EXREF } & 7.26 \mathrm{ppm} \\ \mathrm{BF} & 0.12 \mathrm{~Hz}\end{array}$

RGAIN

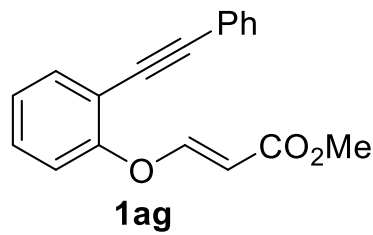

S2 - 14 
single pulse decoupled gated NOE

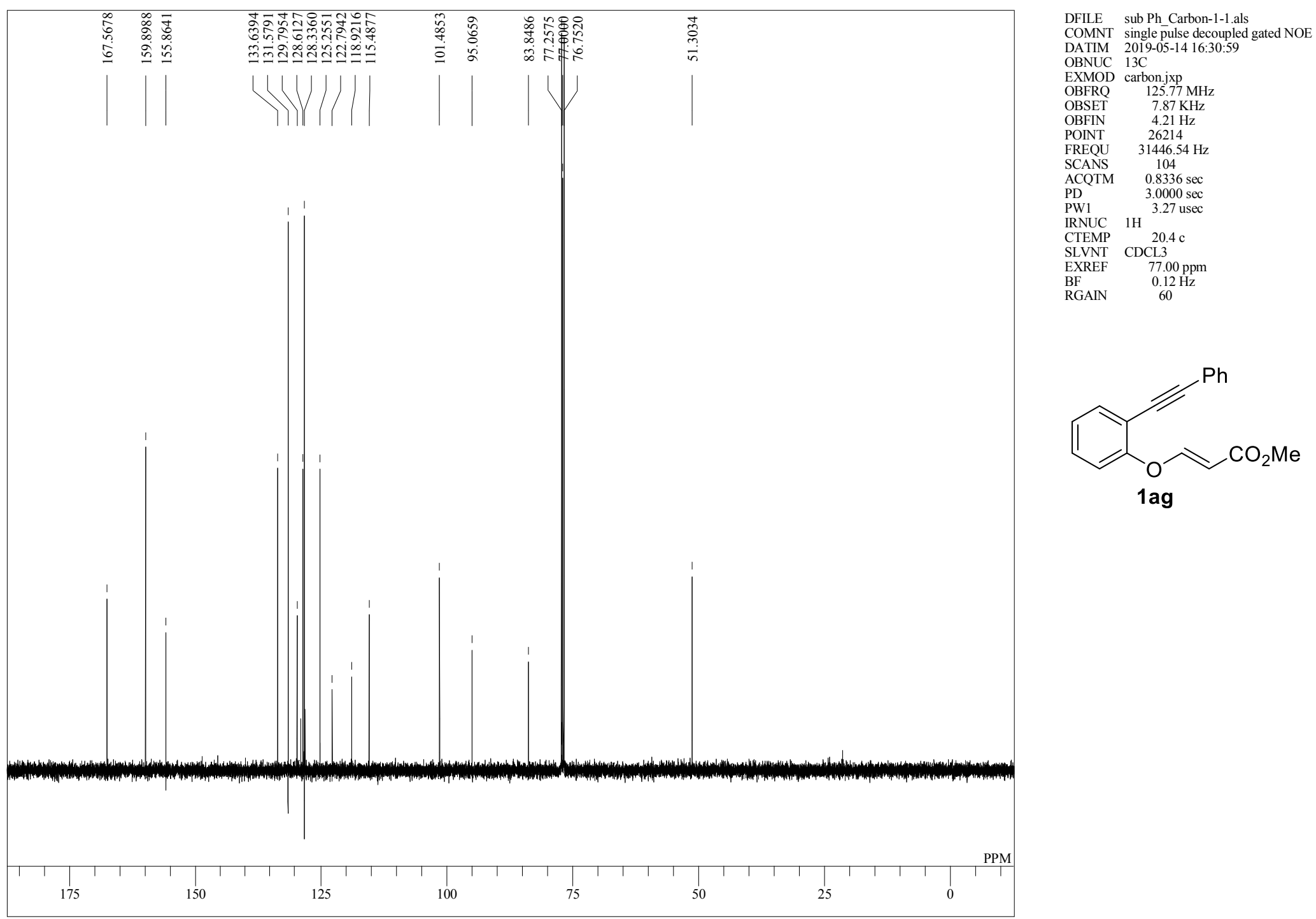


single _pulse

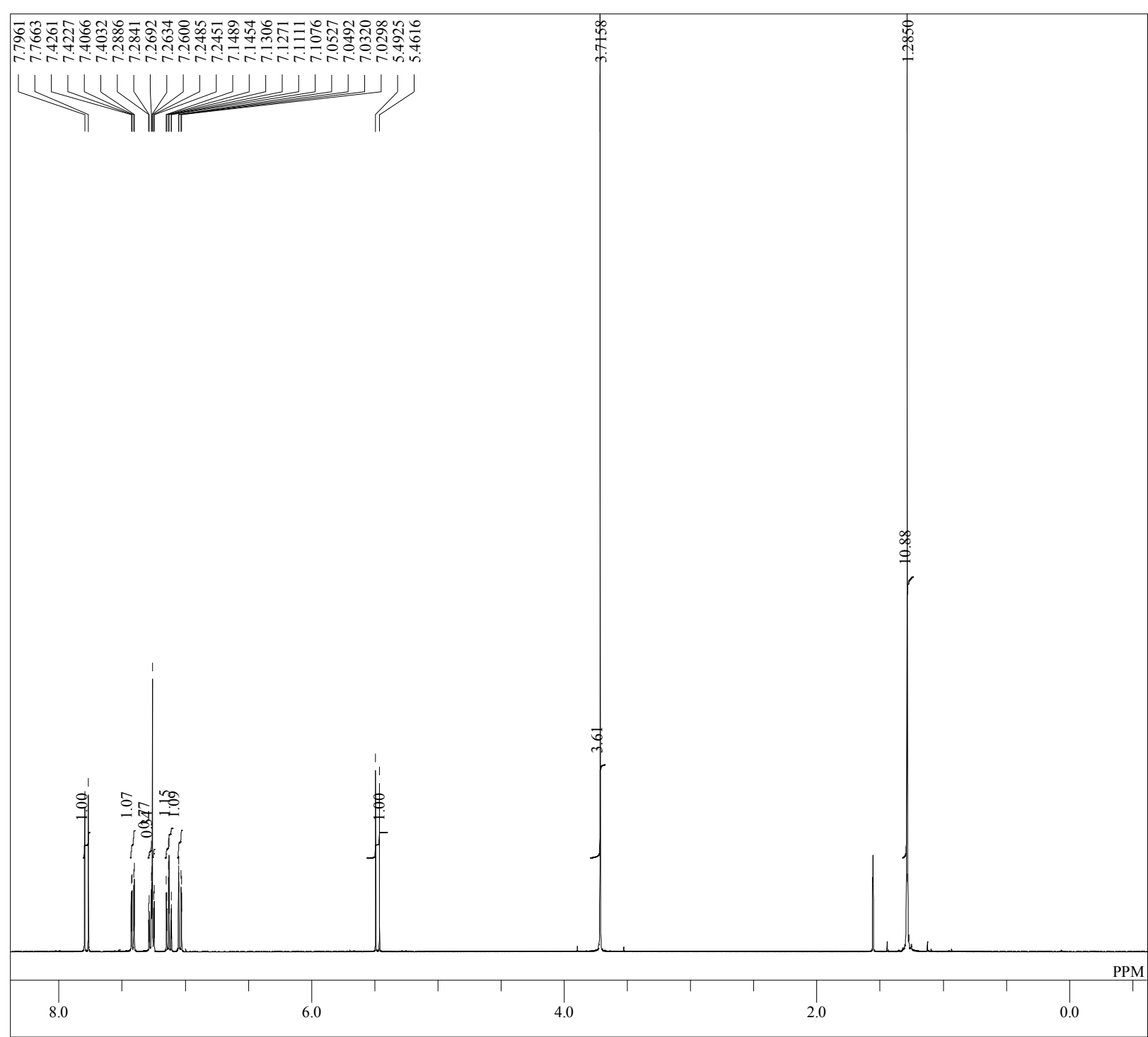

DFILE sub tBu_proton-1-1.als

OBNUC $1 \mathrm{H}$

EXMOD p

OBFRQ $\quad 399.78 \mathrm{MHz}$

$\begin{array}{ll}\text { OBSET } & 4.19 \mathrm{KHz} \\ \text { OBEIN } & 729 \mathrm{~Hz}\end{array}$

POINT 13107

FREQU $\quad 6002.40 \mathrm{~Hz}$

$\begin{array}{ll}\text { SCANS } & 16 \\ \text { ACQTM } & 2.1837 \mathrm{sec}\end{array}$

$\begin{array}{ll}\text { ACQTM } & 2.1837 \mathrm{sec} \\ \mathrm{PD} & 1.0000 \mathrm{sec}\end{array}$

$\begin{array}{ll}\text { PW1 } & 1.000 \mathrm{sec} \\ \text { PW1 } & 7.25 \mathrm{usec}\end{array}$

$\begin{array}{ll}\text { IRNUC } & 1 \mathrm{H} \\ \text { CTEMP } & \\ 19.6 \mathrm{c}\end{array}$

$\begin{array}{lr}\text { CTEMP } & 19.6 \mathrm{c} \\ \text { SLVNT } & \text { CDCL3 }\end{array}$

$\begin{array}{ll}\text { EXREF } & 7.26 \mathrm{ppm} \\ \mathrm{BF} & 0.12 \mathrm{~Hz}\end{array}$

$\begin{array}{ll}\text { BF } & 0.12 \mathrm{~Hz} \\ \text { RGAIN } & 50\end{array}$

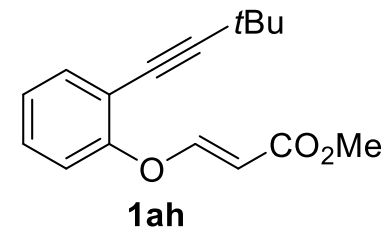

$\mathrm{S} 2-16$ 
single pulse decoupled gated NOE

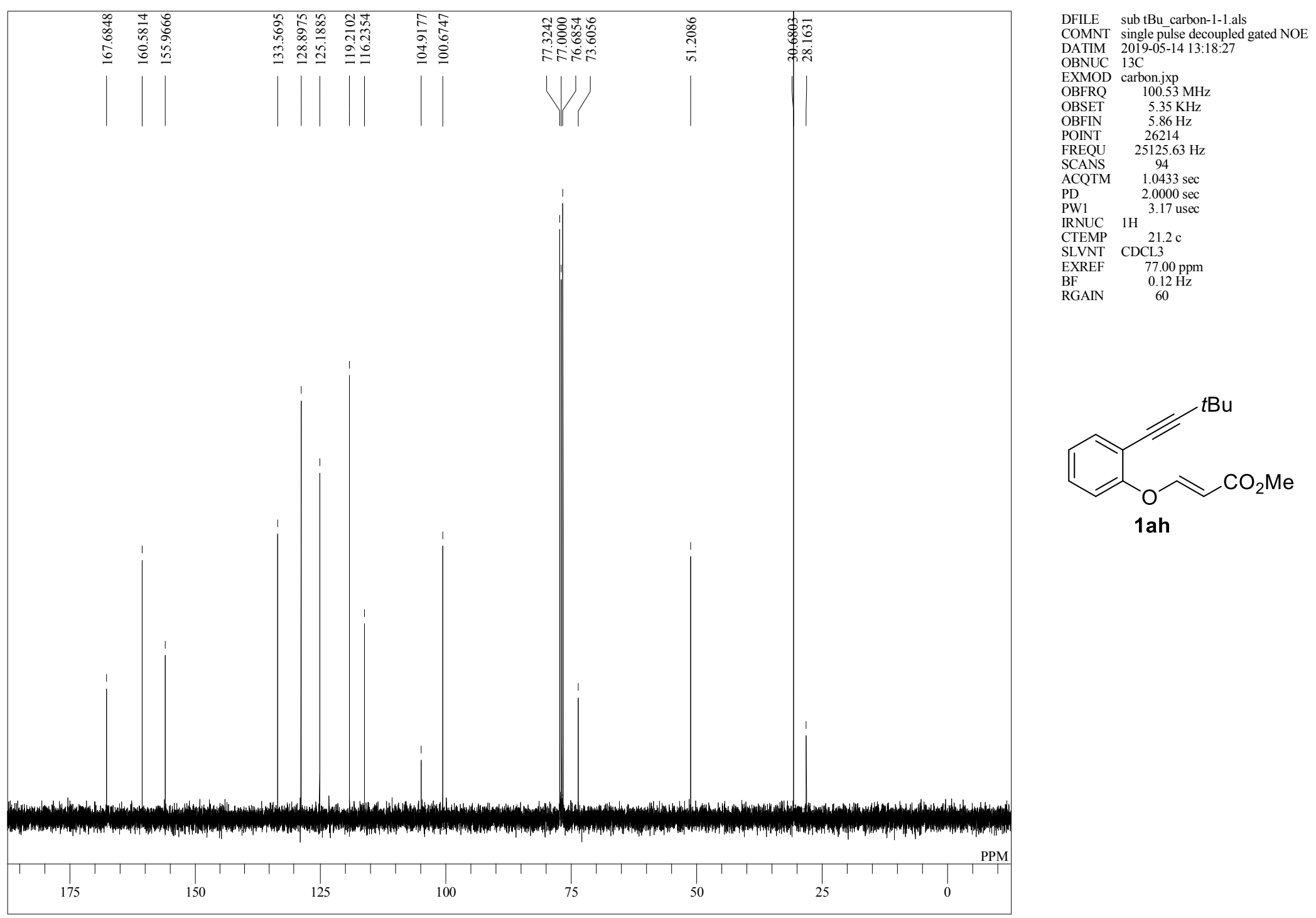

S2 - 17 
single _pulse

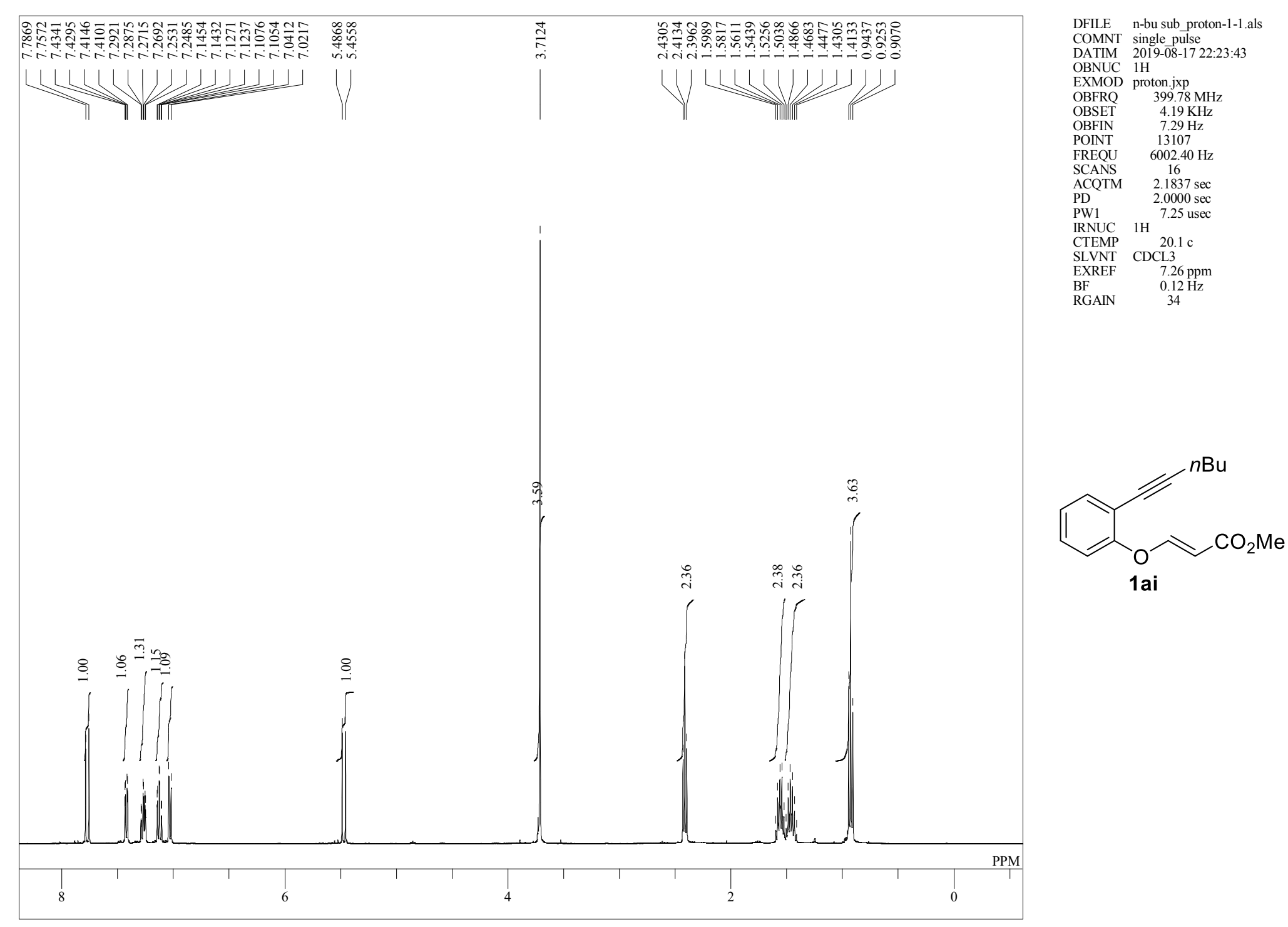


single pulse decoupled gated NOE

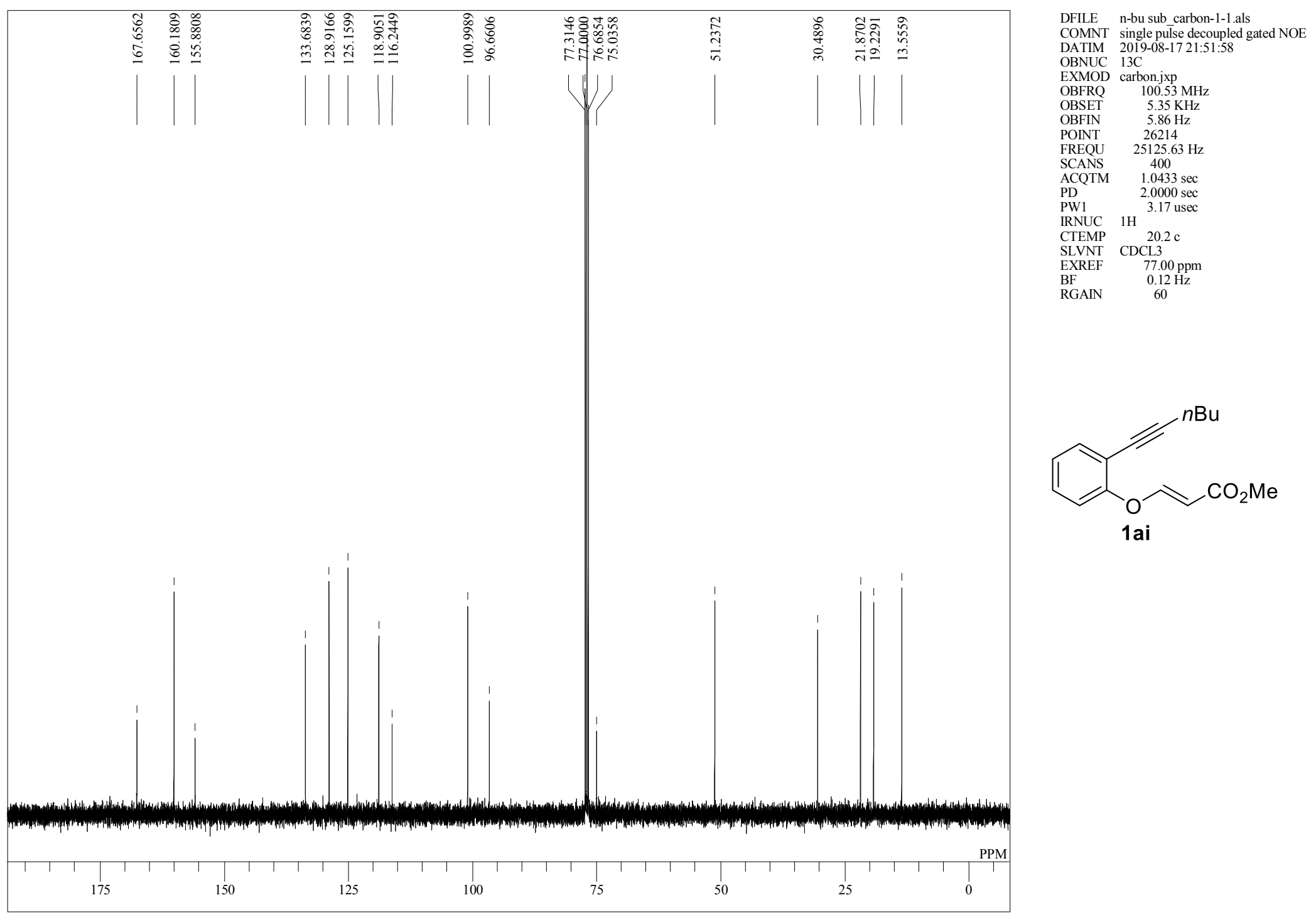

S2 - 19 
single pulse

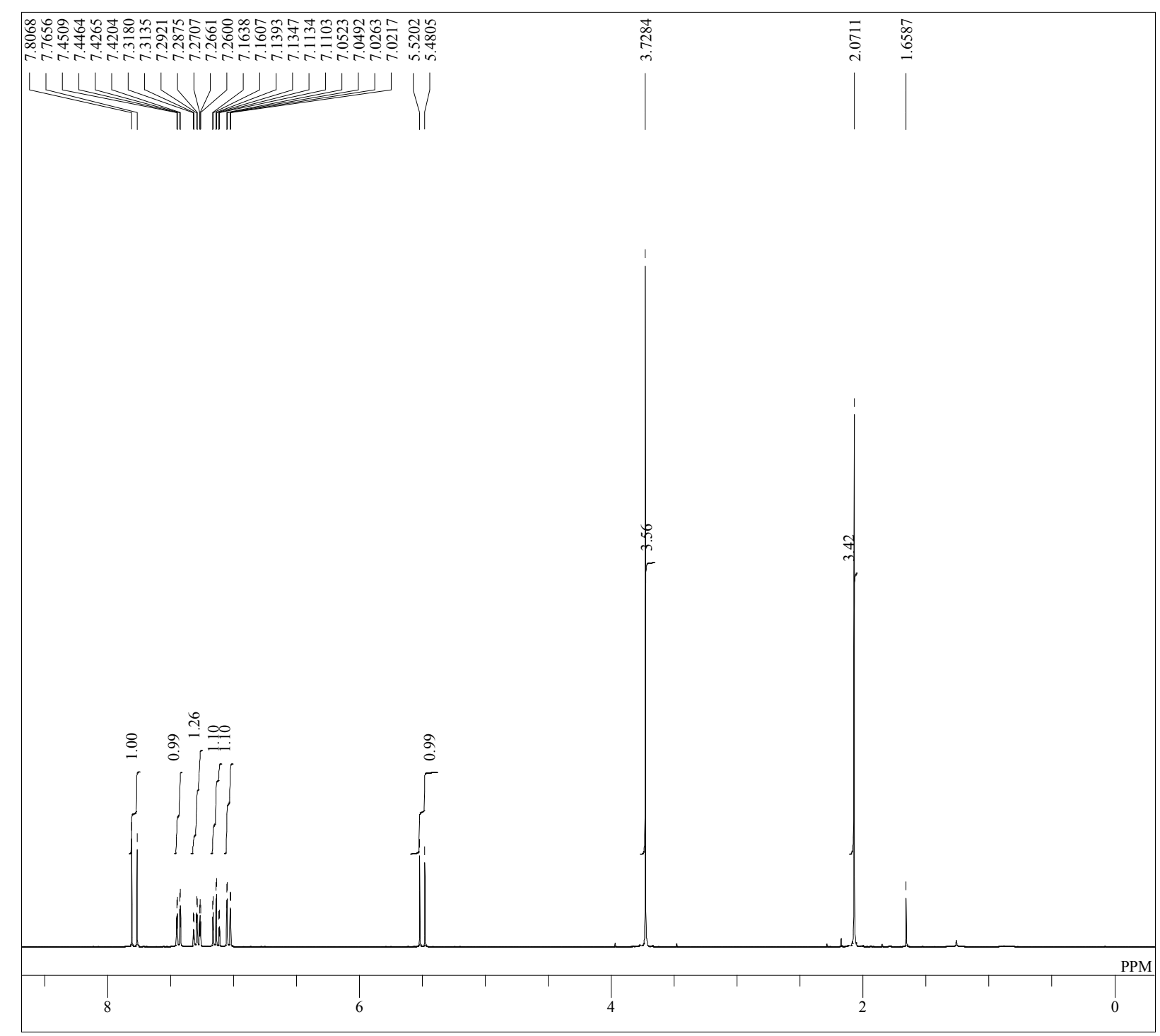

DFILE sub Me_proton-1-1.als

DATIM 2019-05-14 16:10:13

OBNUC $1 \mathrm{H}$

$\begin{array}{ll}\text { EXMOD } & \text { proton.jxp } \\ \text { OBFRQ } & 300.53 \mathrm{MH} z\end{array}$

OBSET $\quad 1.15 \mathrm{KHz}$

POINT 13107

FREQU $\quad 6016.85 \mathrm{~Hz}$

$\begin{array}{ll}\text { SCANS } & 16 \\ & \text { ACQTM }\end{array}$

$\mathrm{PD} \quad-1.1784 \mathrm{sec}$

PW1 $\quad 5.50 \mathrm{usec}$

IRNUC $1 \mathrm{H}$

$\begin{array}{lr}\text { CTEMP } & 20.1 \mathrm{c} \\ \text { SLVNT } & \text { CDCL3 }\end{array}$

$\begin{array}{ll}\text { EXREF } & 7.26 \mathrm{ppm} \\ \mathrm{BF} & 0.12 \mathrm{~Hz}\end{array}$

RGAIN

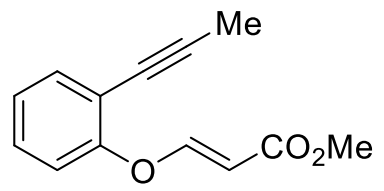

1aj 
single pulse decoupled gated NOE

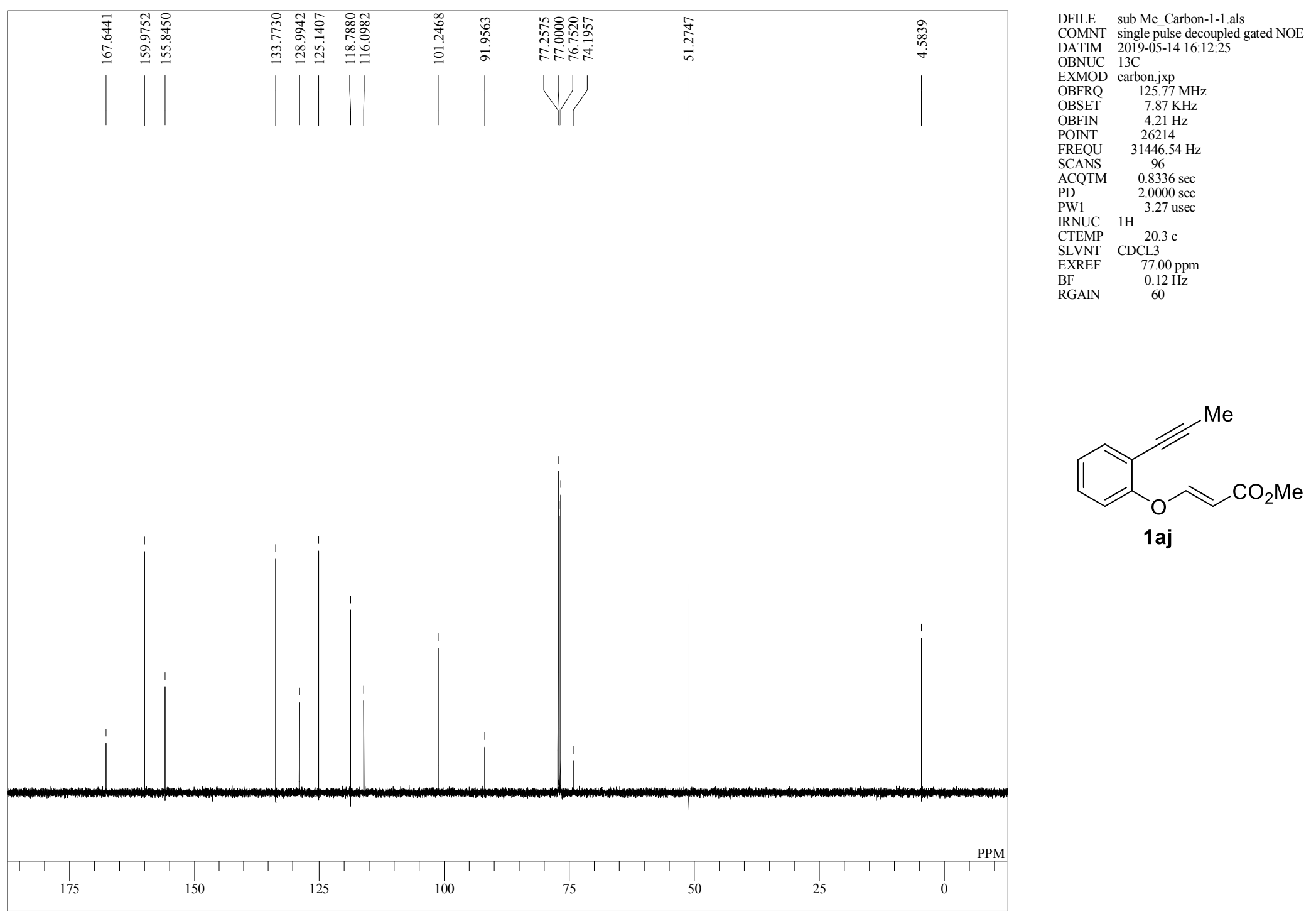


single pulse

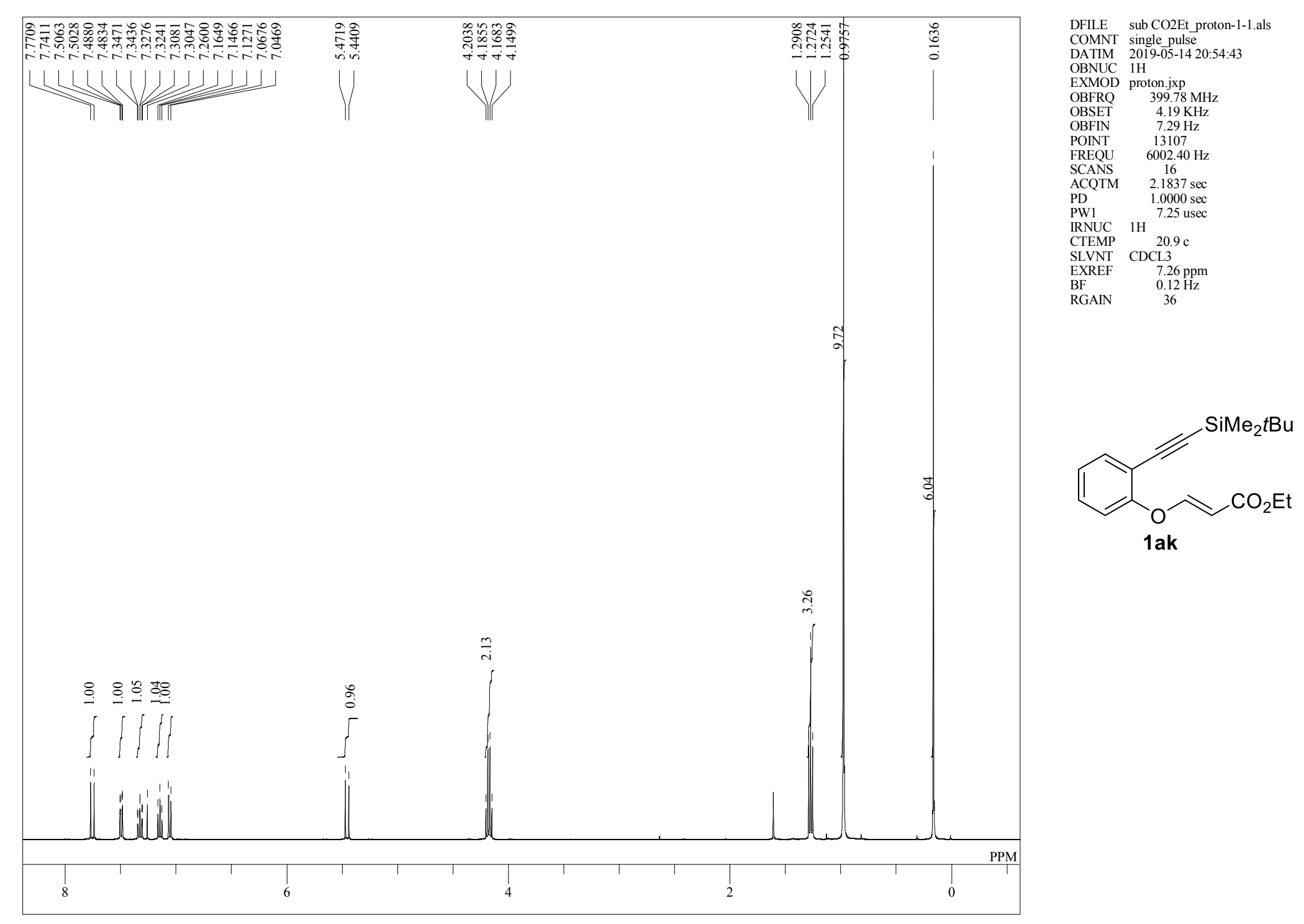


single pulse decoupled gated NOE

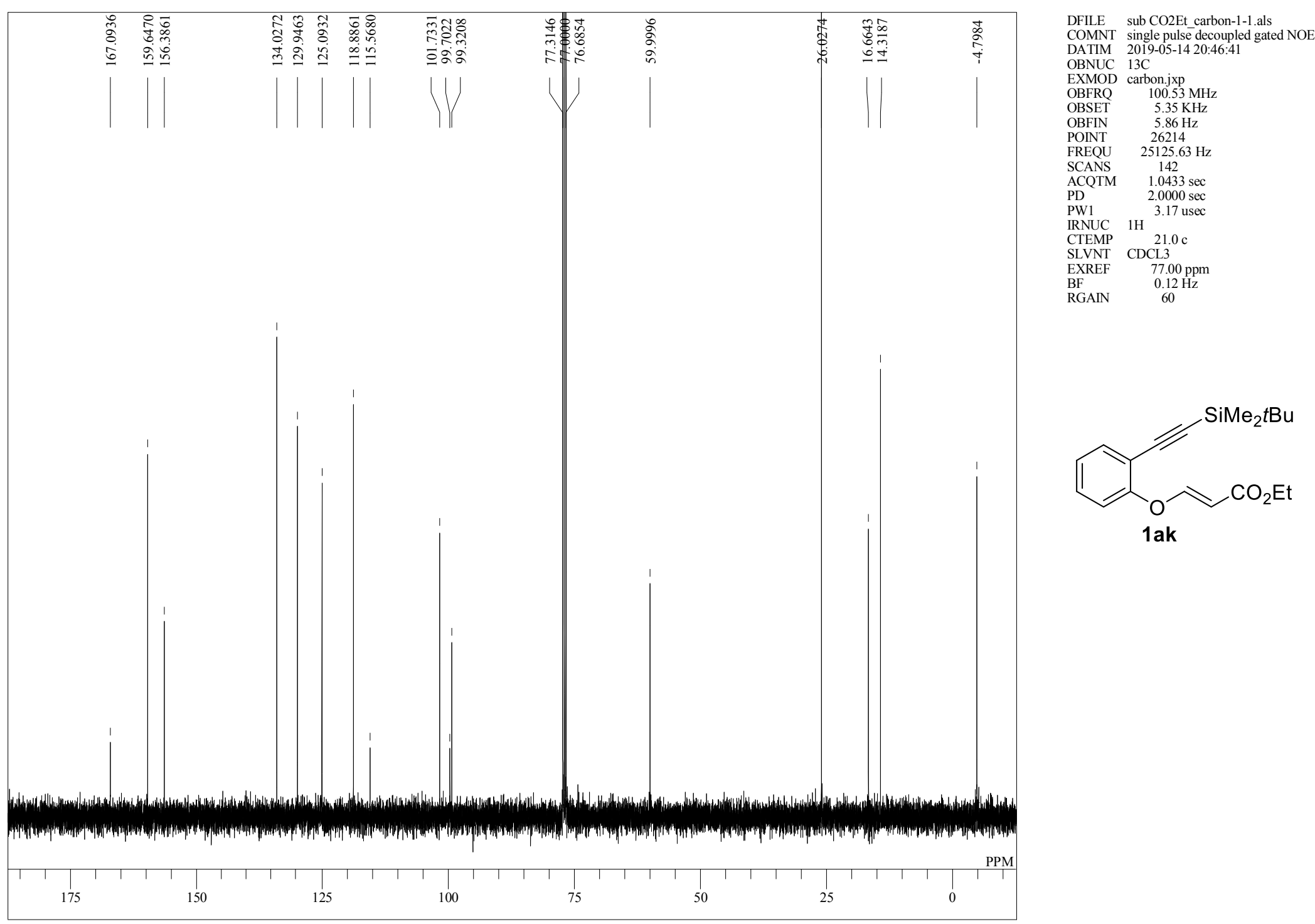


single pulse

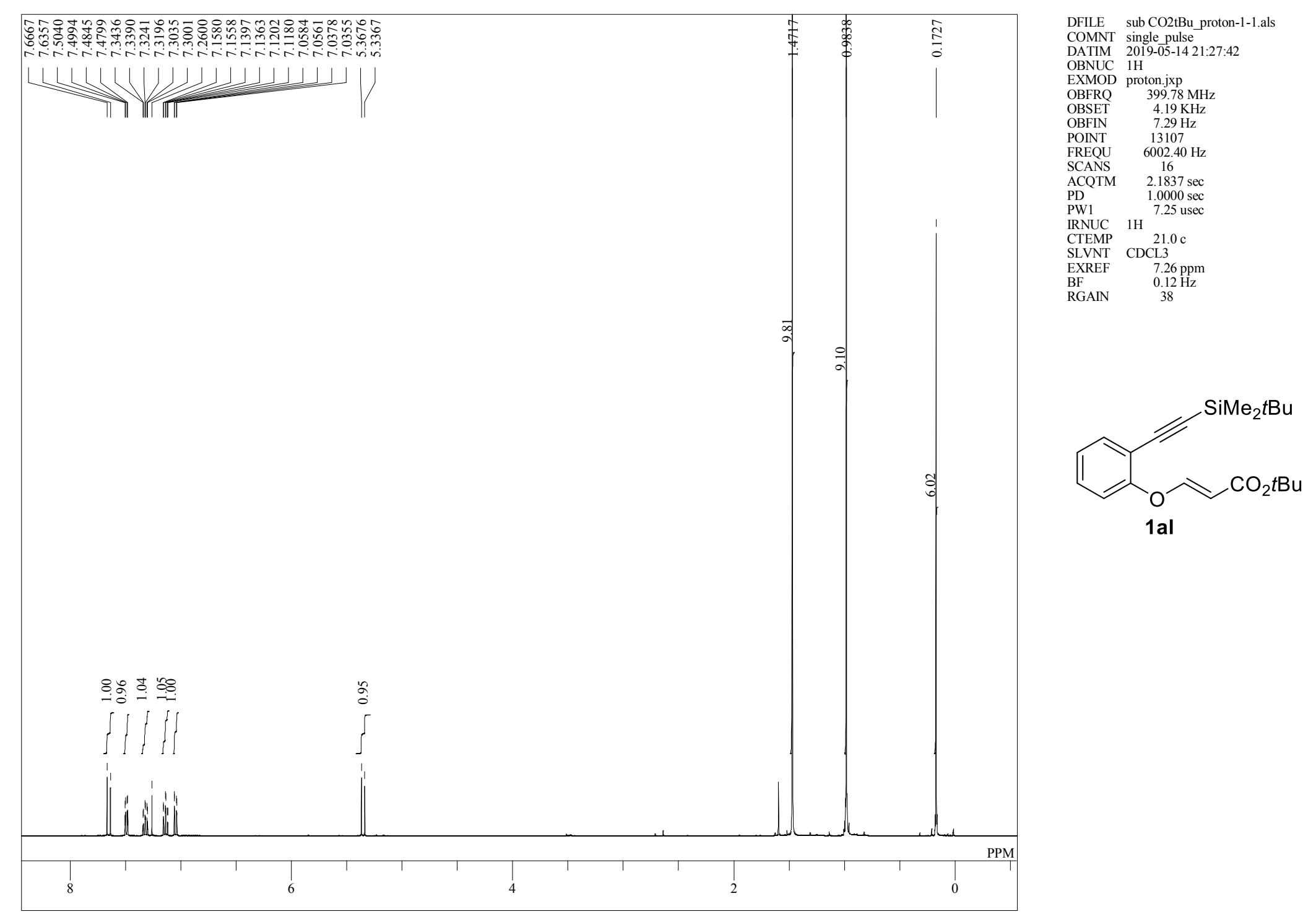


single pulse decoupled gated NOE

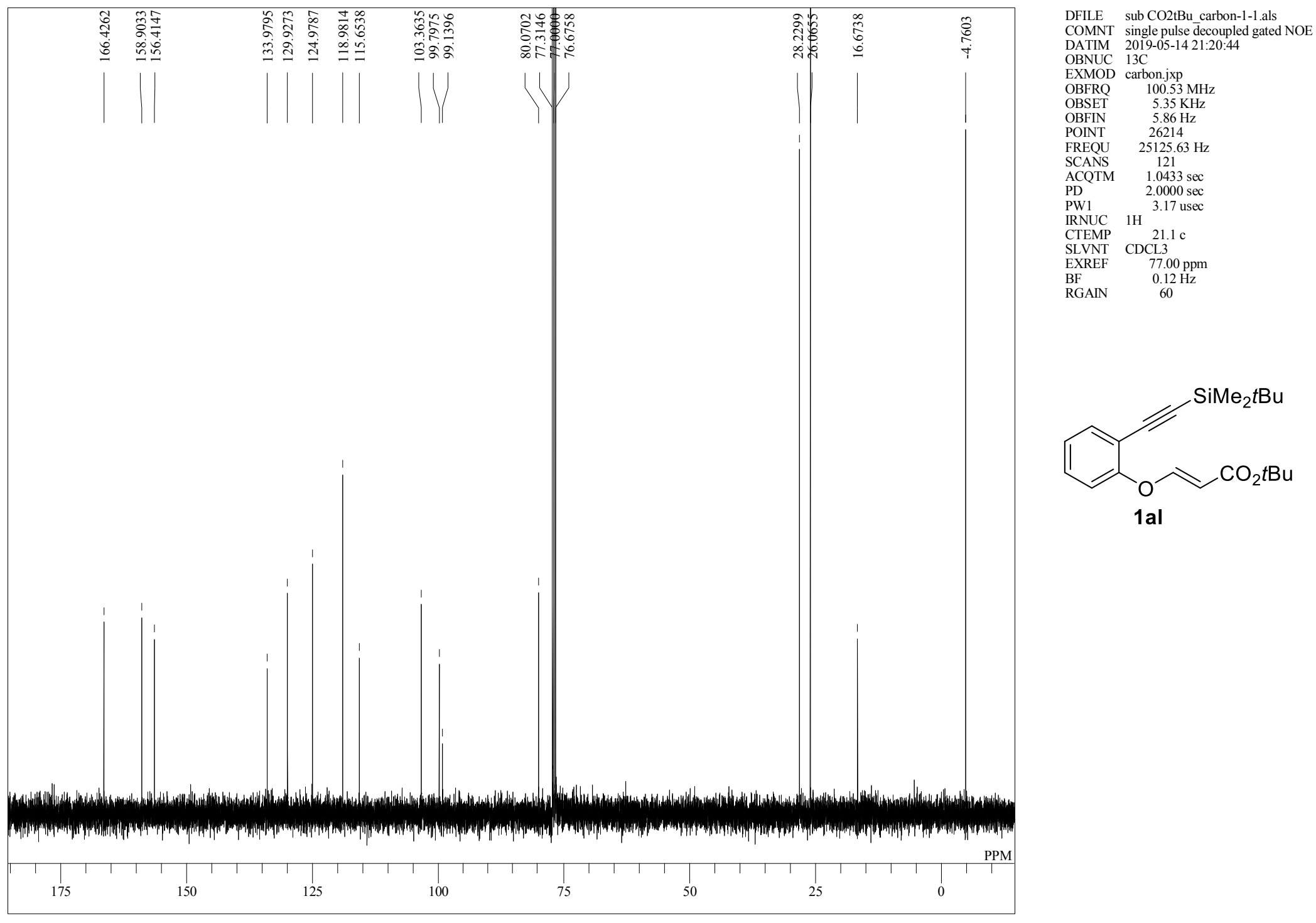


single pulse

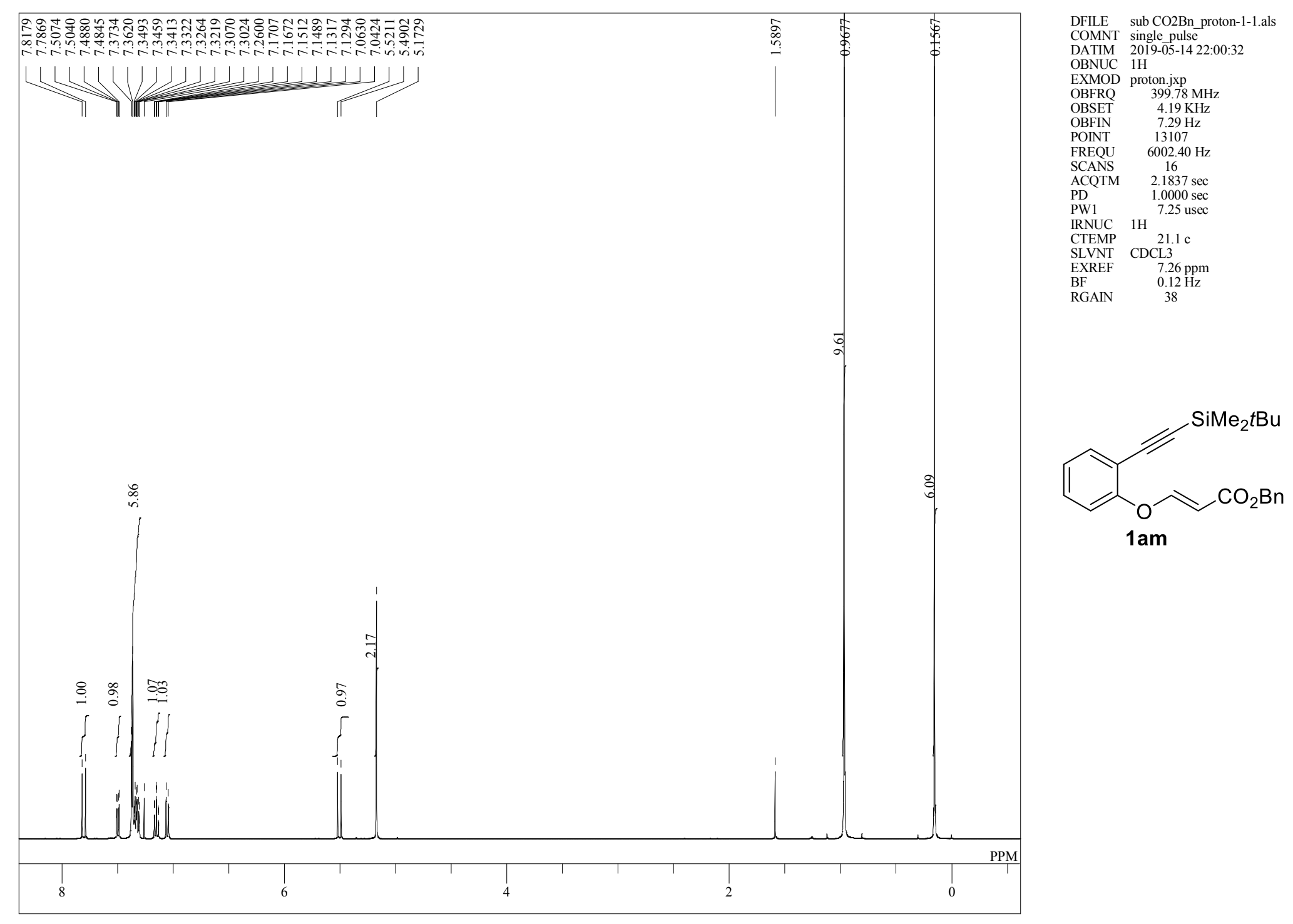


single pulse decoupled gated NOE

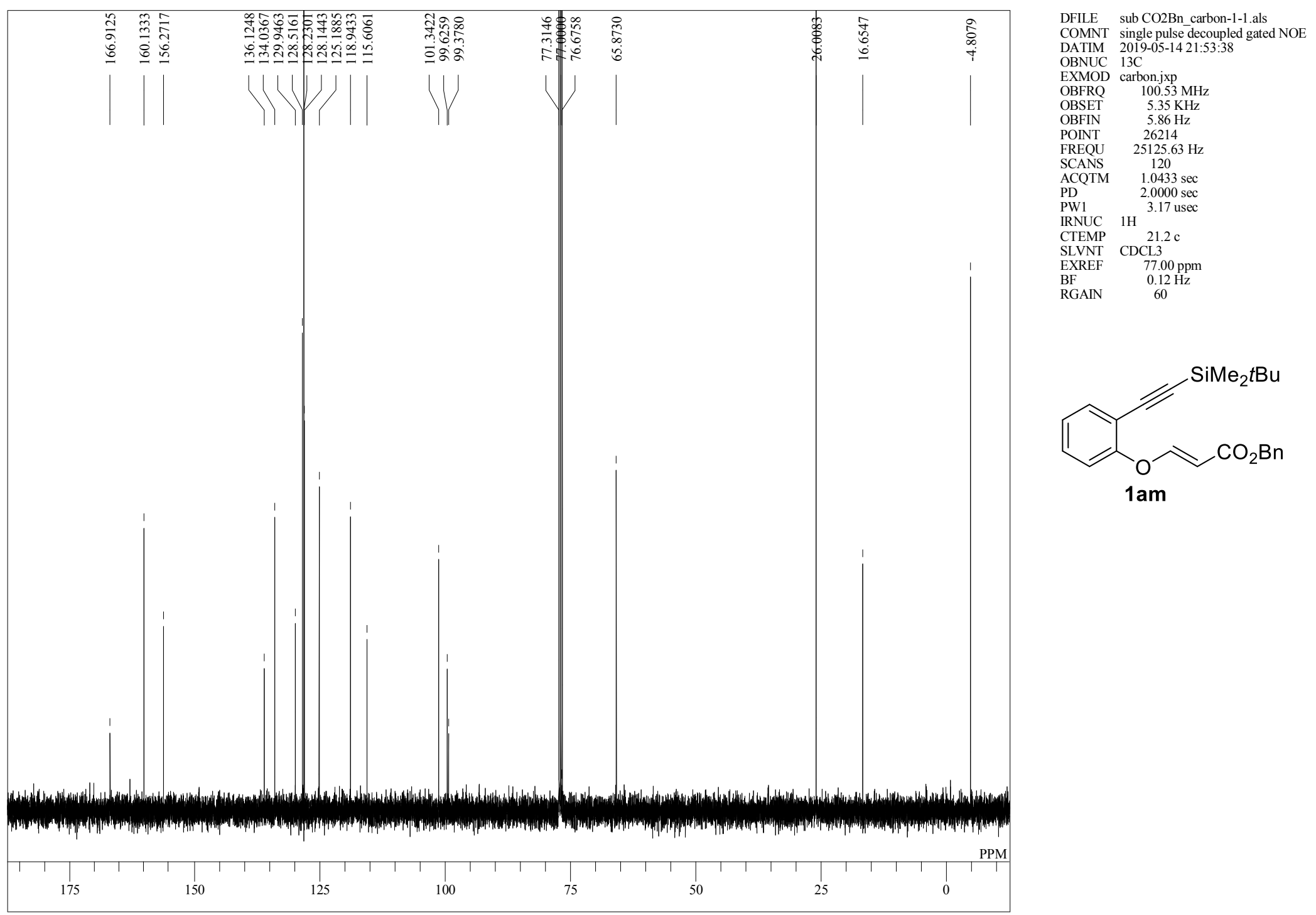


single pulse

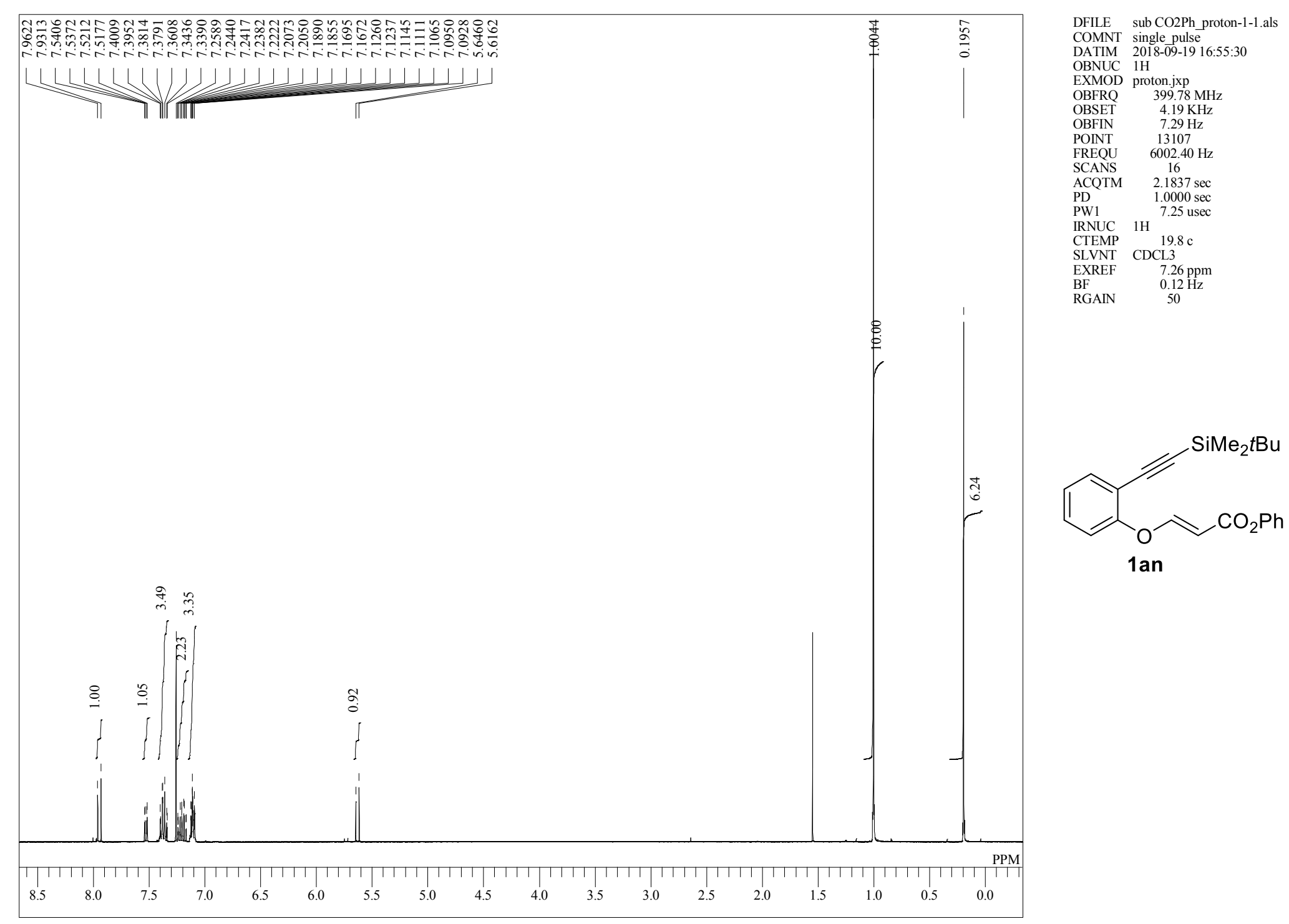


single pulse decoupled gated NOE

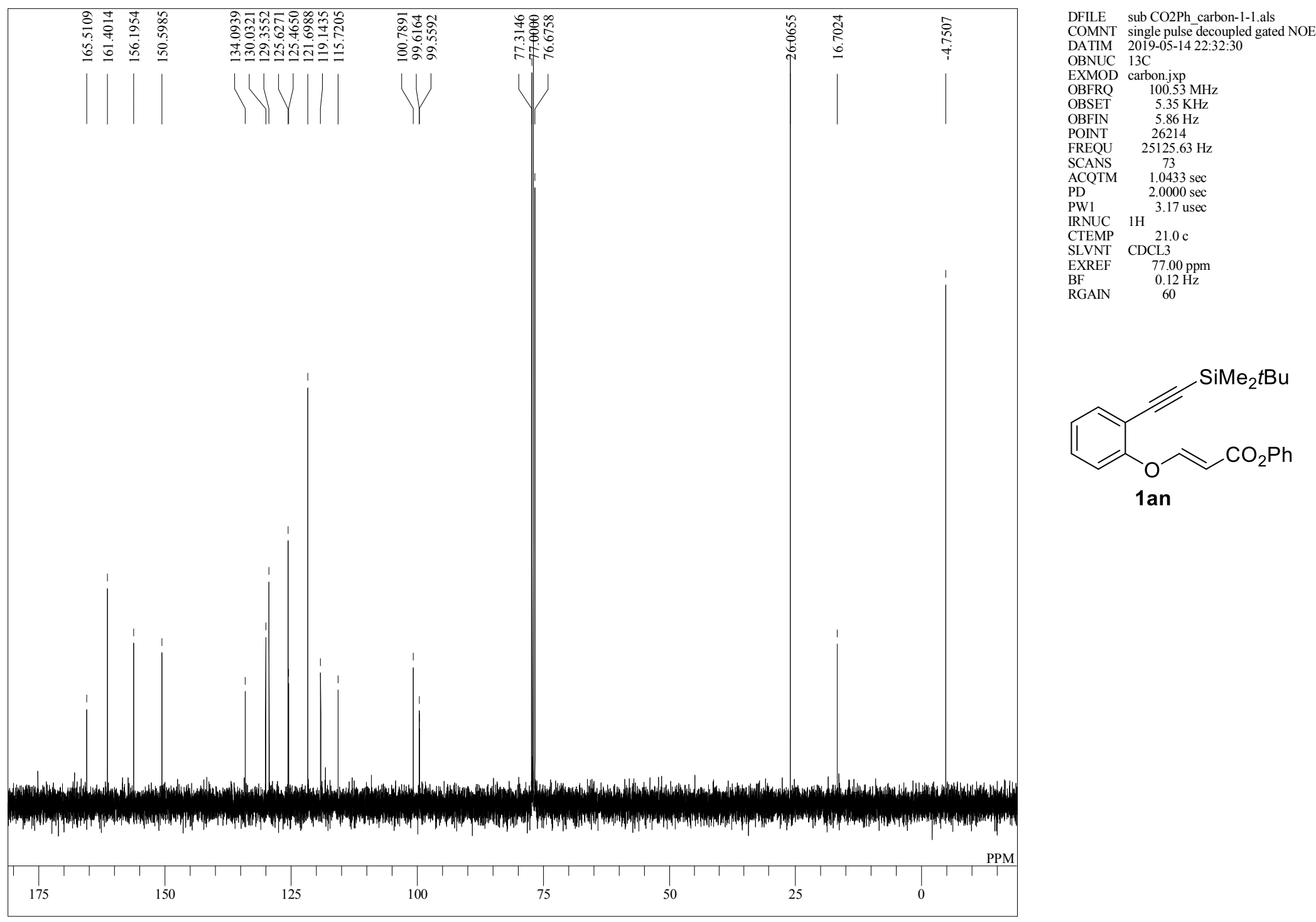

S2 - 29 
single pulse

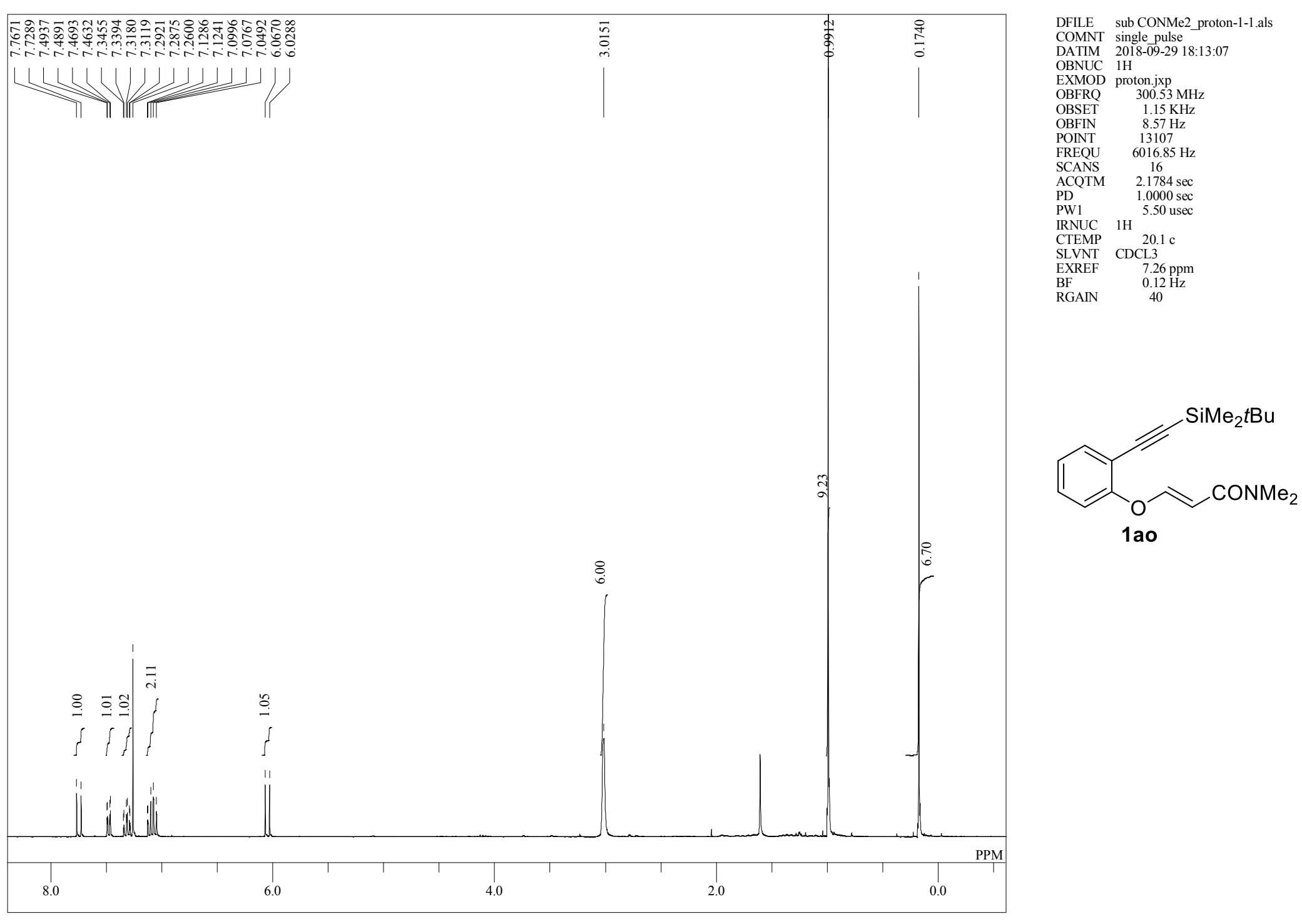

$\mathrm{S} 2-30$ 
single pulse decoupled gated NOE

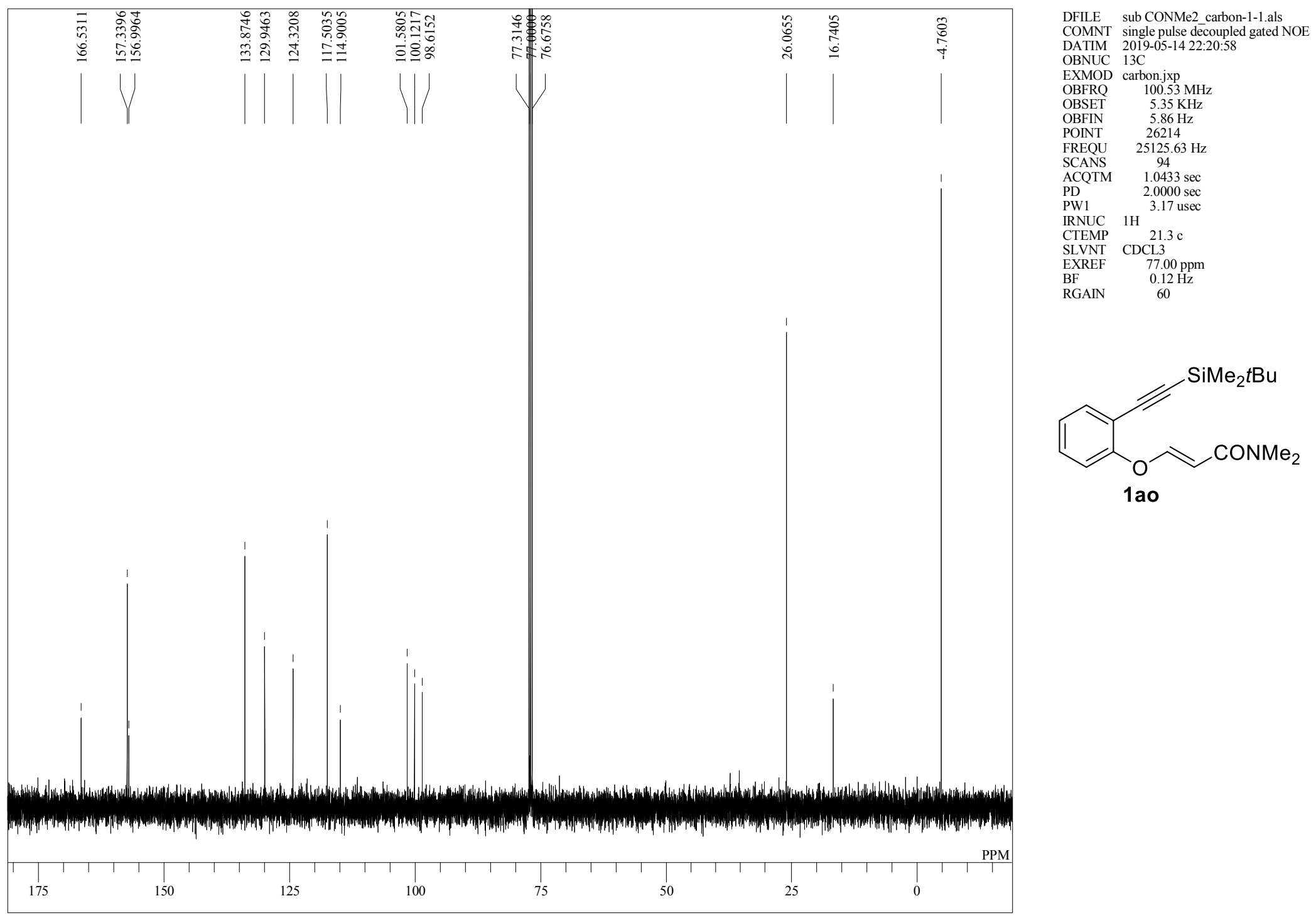


single pulse

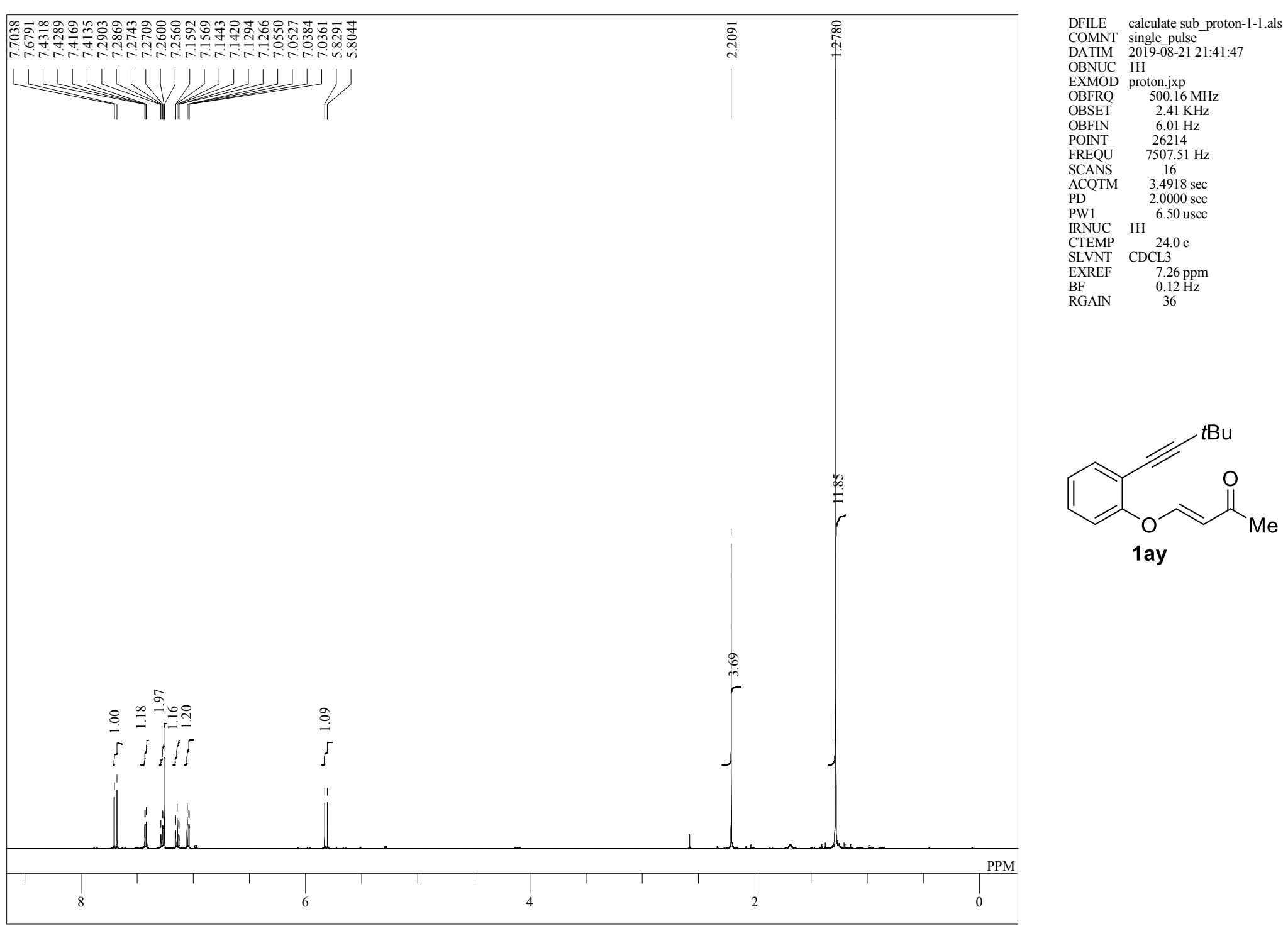

$\mathrm{S} 2-32$ 
single pulse decoupled gated NOE

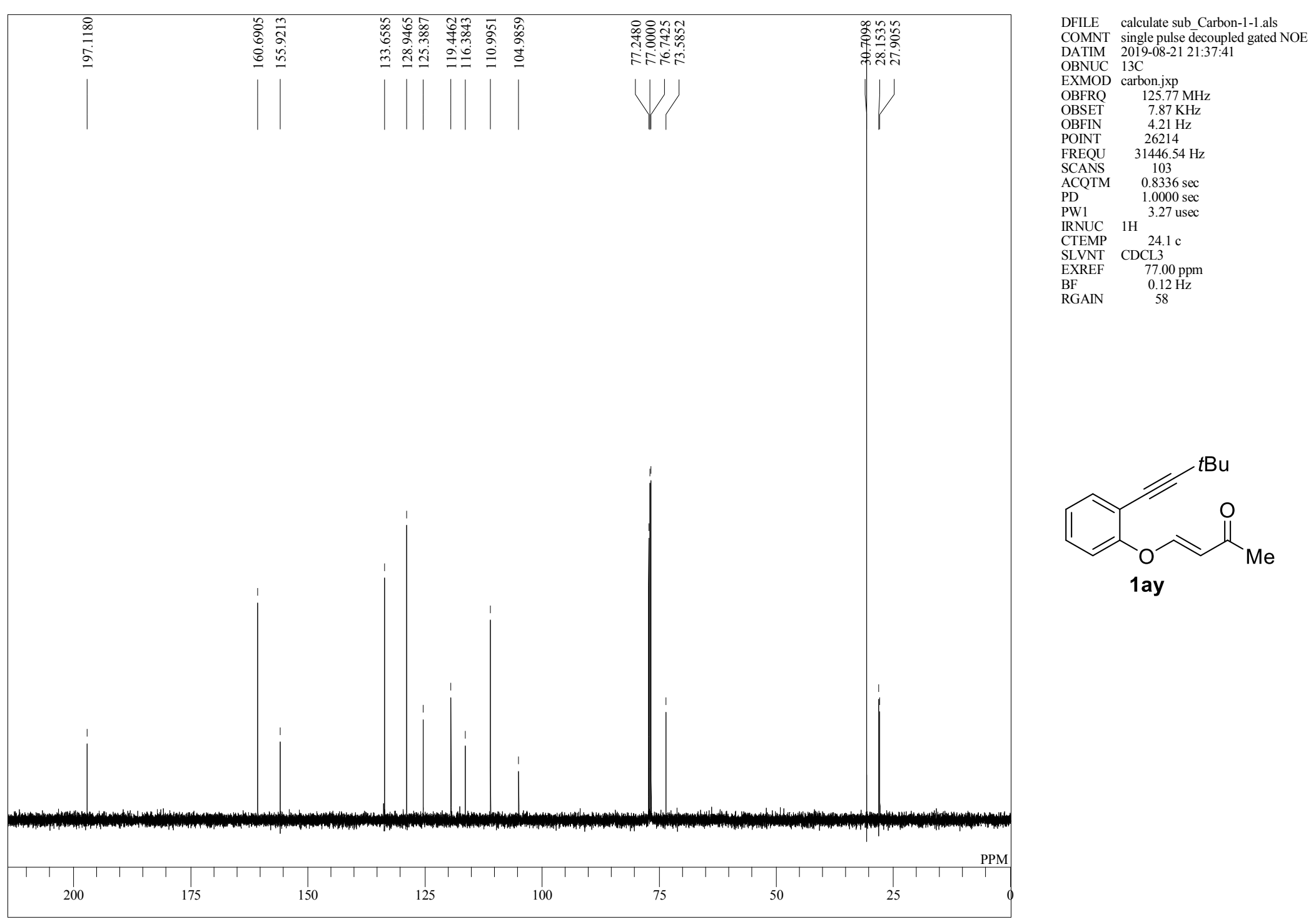


single pulse

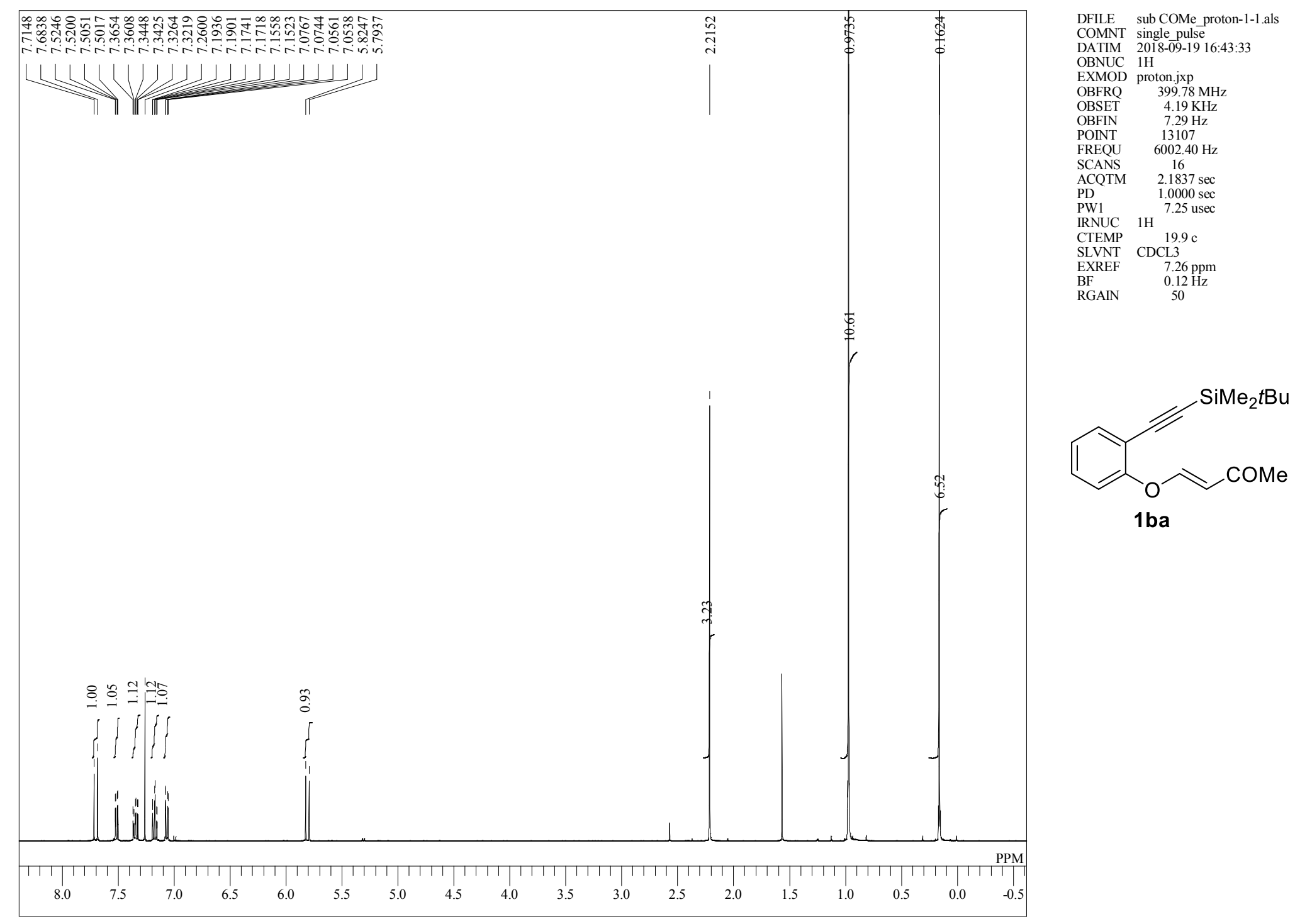


single pulse decoupled gated NOE

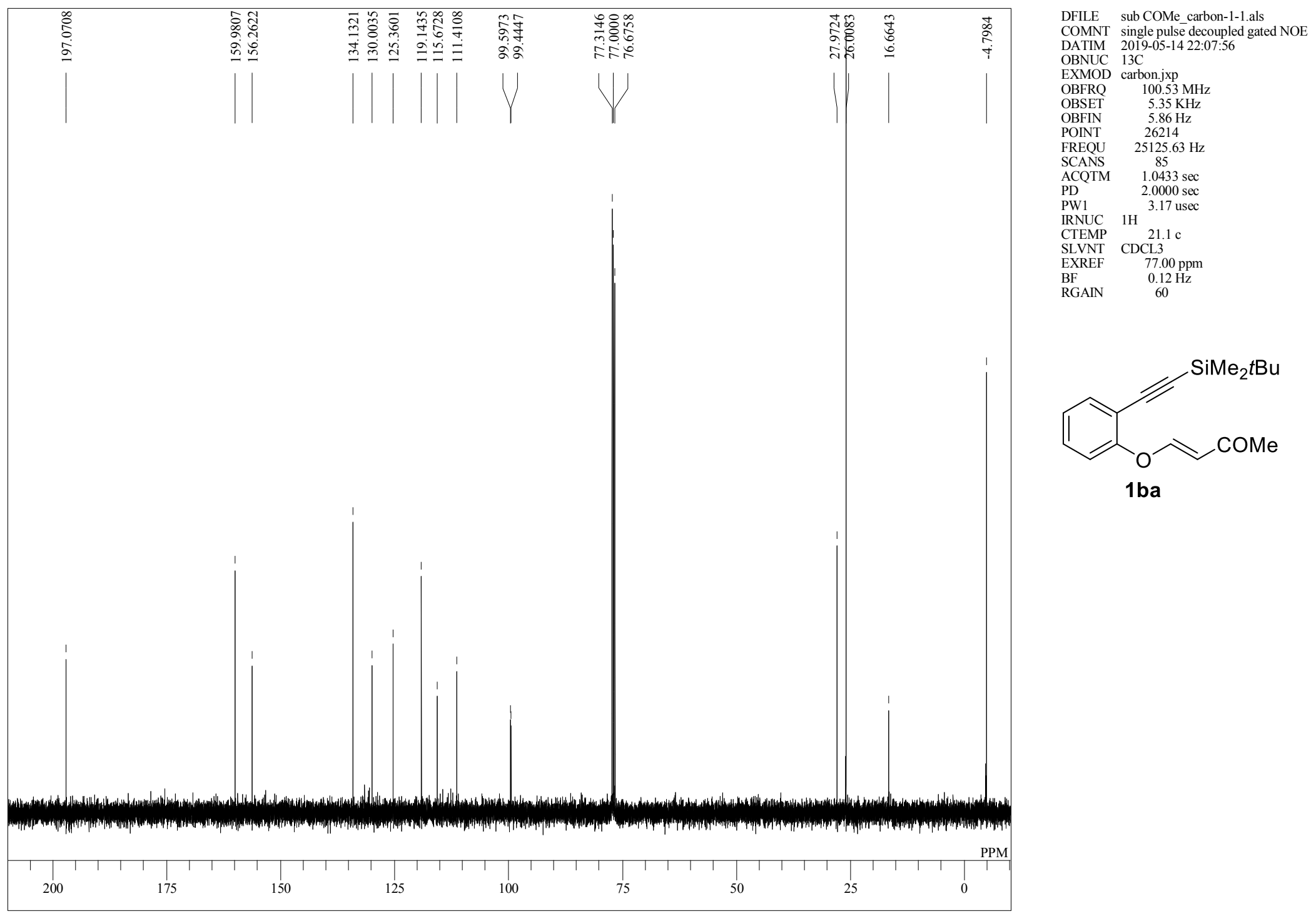




\section{single pulse}

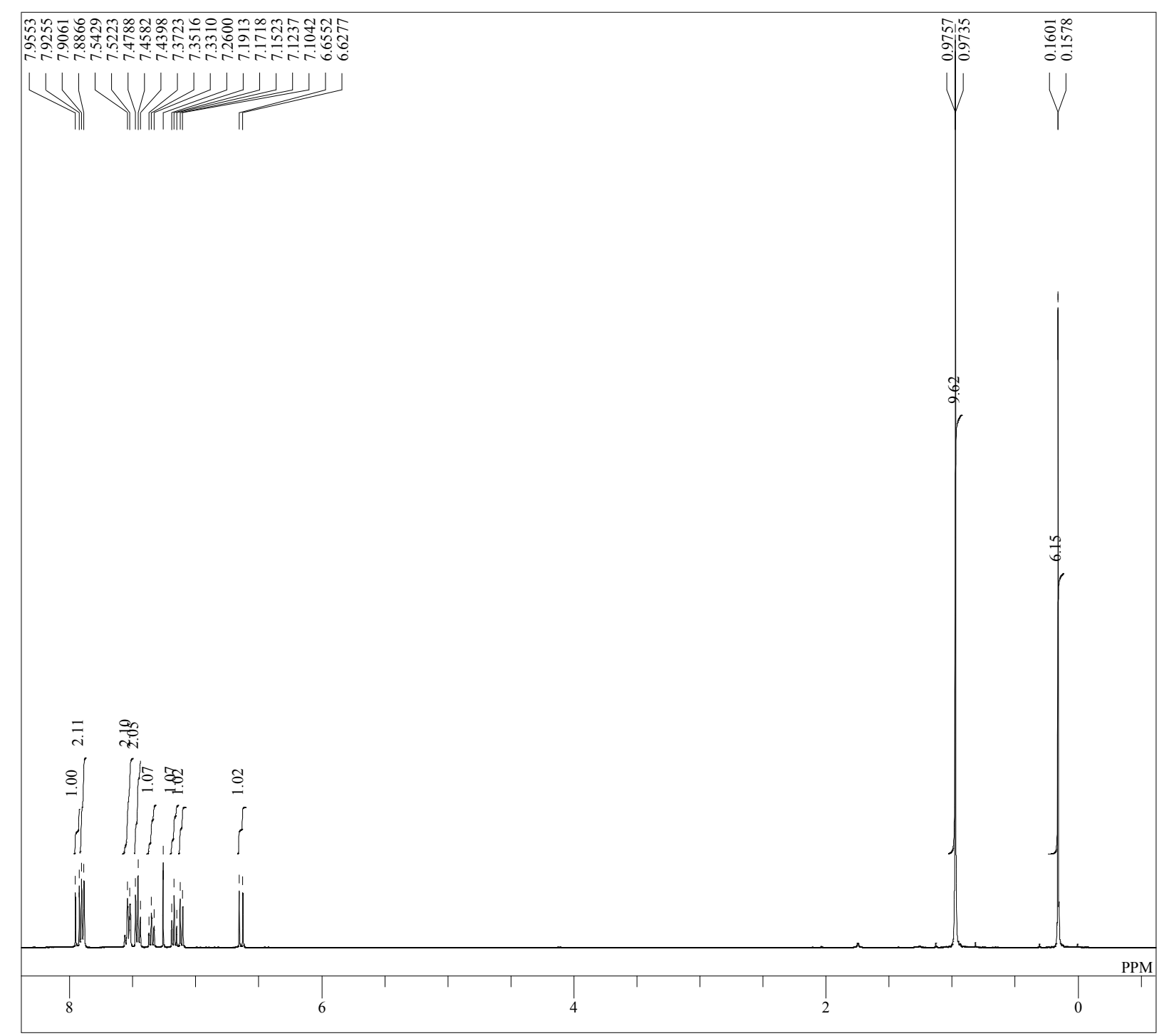

DFILE sub coph_proton-1-1.als

COMNT single pulse

OBNUC $1 \mathrm{H}$

EXMOD proton.jxp
OBFRO $399.78 \mathrm{MH} z$

OBSET $\quad 4.19 \mathrm{KHz}$

$\begin{array}{ll}\text { OBFIN } & 7.29 \mathrm{~Hz} \\ \text { POINT } & 13107\end{array}$

FREQU $\quad 6002.40 \mathrm{~Hz}$

SCANS 16

ACQTM $\quad 2.1837 \mathrm{sec}$

\begin{tabular}{ll} 
PW1 & $2.0000 \mathrm{sec}$ \\
\hline & $7.25 \mathrm{usec}$
\end{tabular}

IRNUC $1 \mathrm{H} .25$ usec

CTEMP $23.2 \mathrm{c}$

$\begin{array}{lc}\text { EXREF } & 7.26 \mathrm{ppm} \\ \text { BF } & 0.12 \mathrm{~Hz} \\ \text { RGAIN } & 28\end{array}$

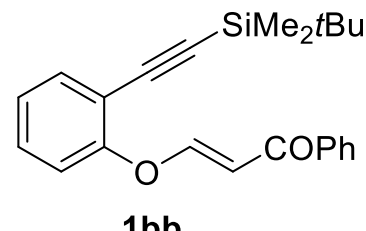

$1 \mathrm{bb}$ 
single pulse decoupled gated NOE

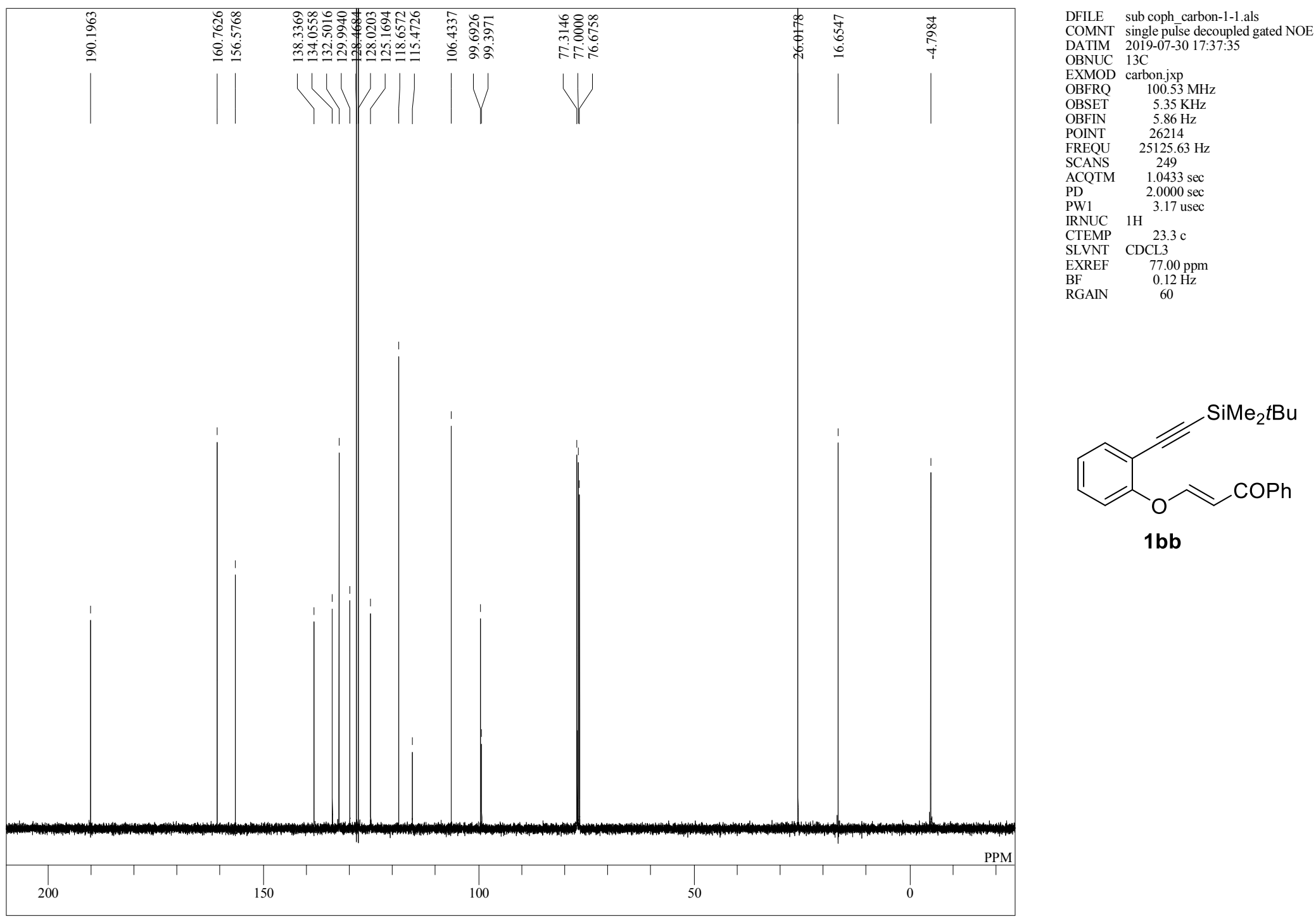


single pulse

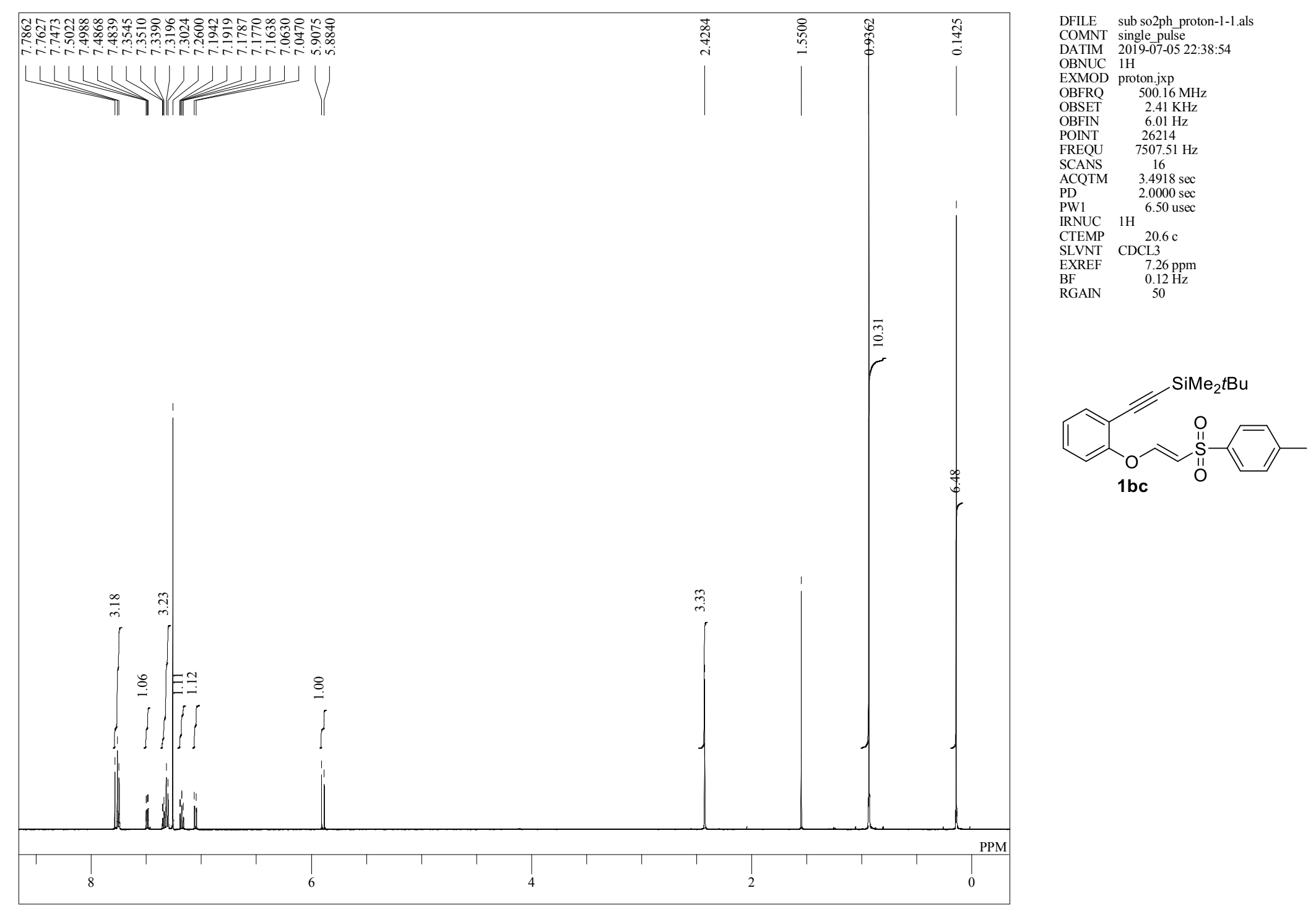


single pulse decoupled gated NOE

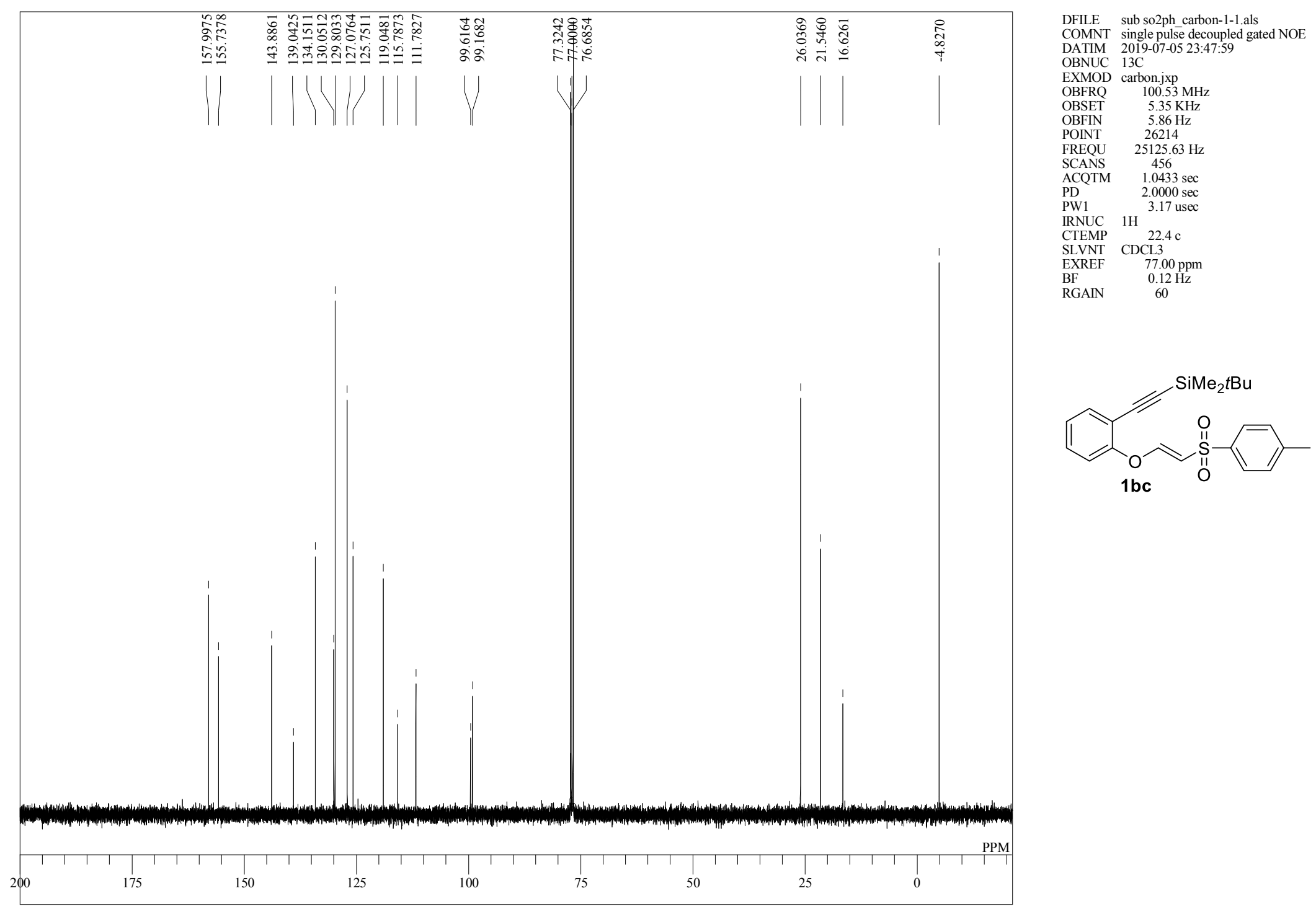

S2 - 39 
single pulse

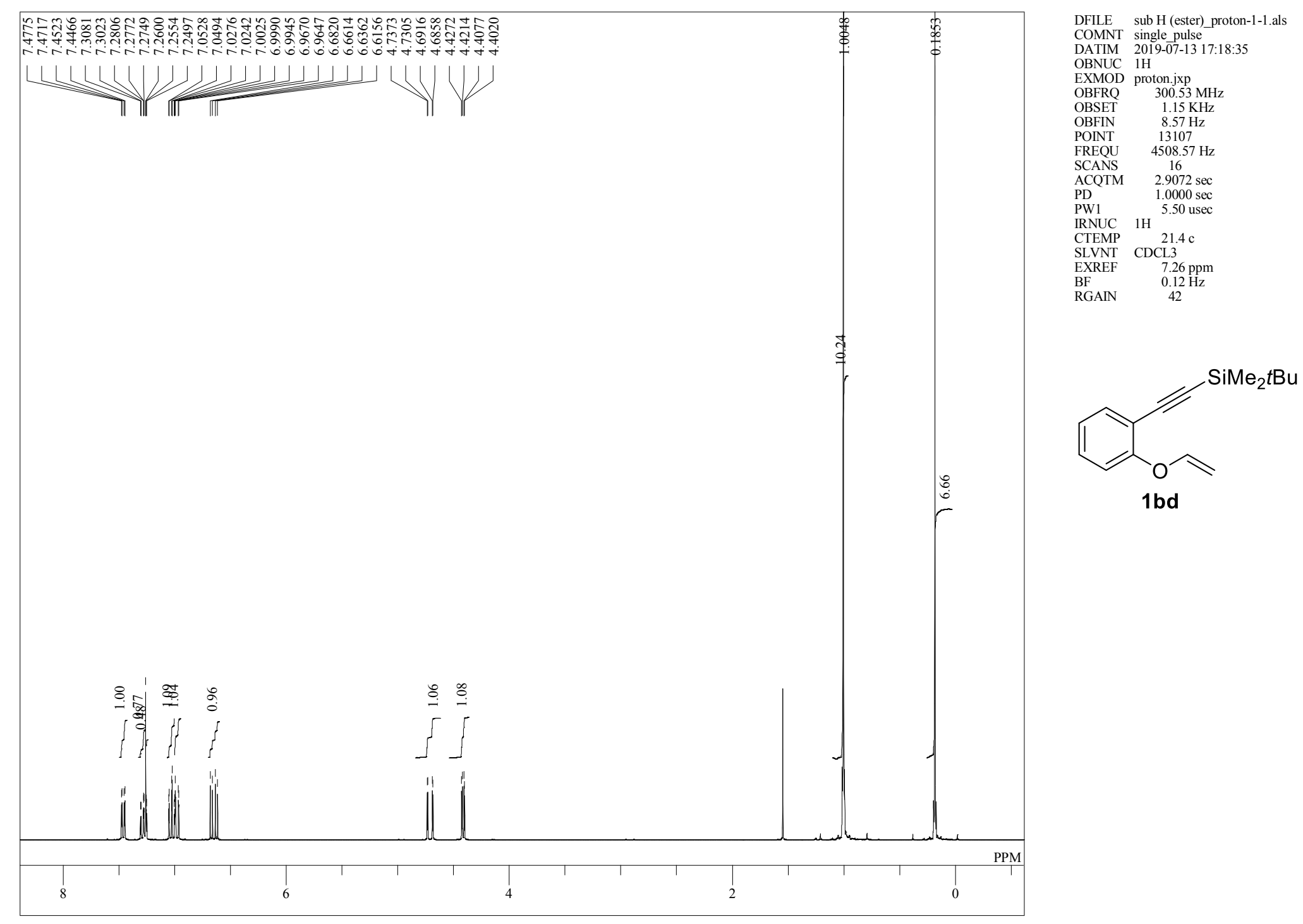

$\mathrm{S} 2-40$ 
single pulse decoupled gated NOE

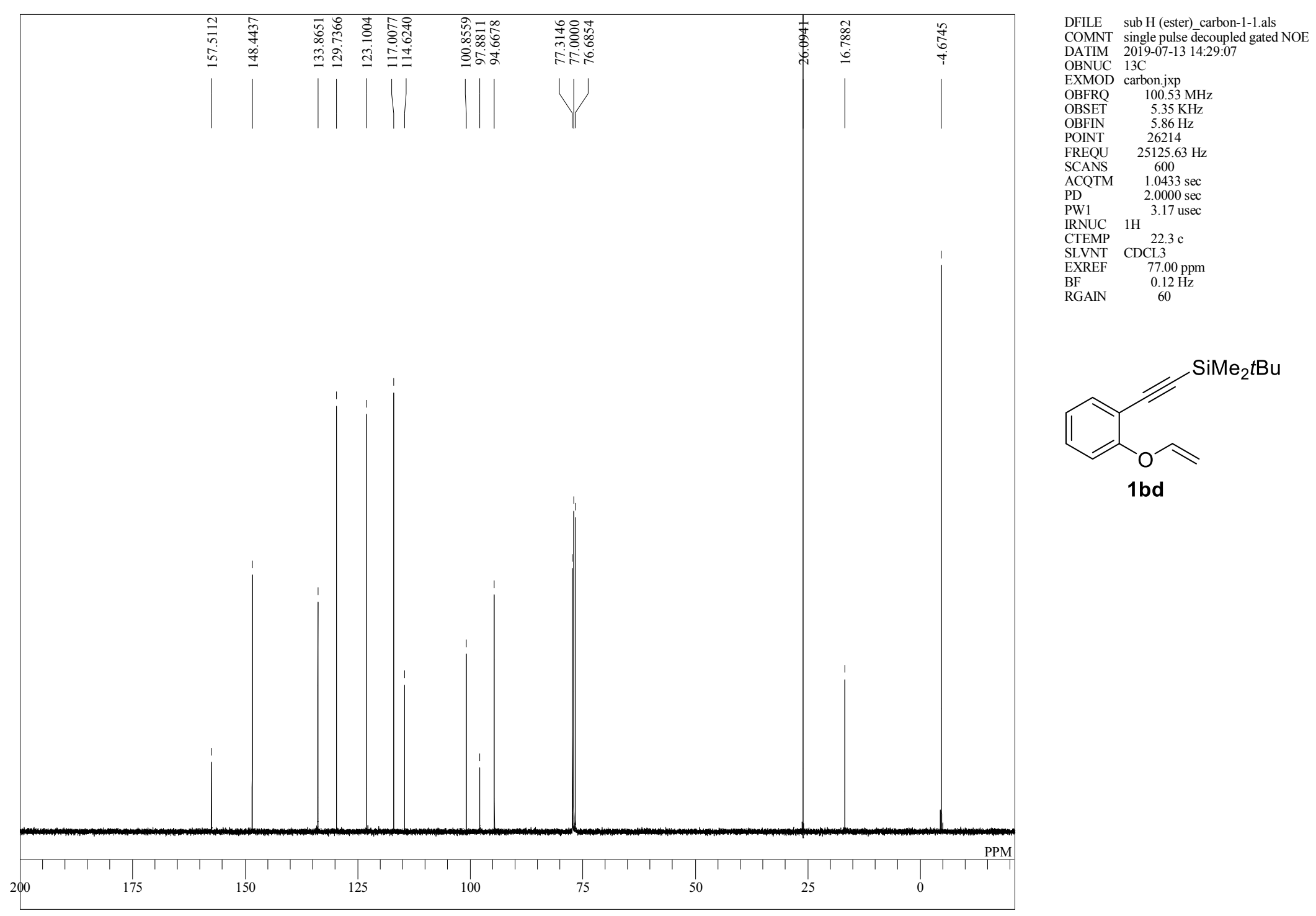


single _pulse

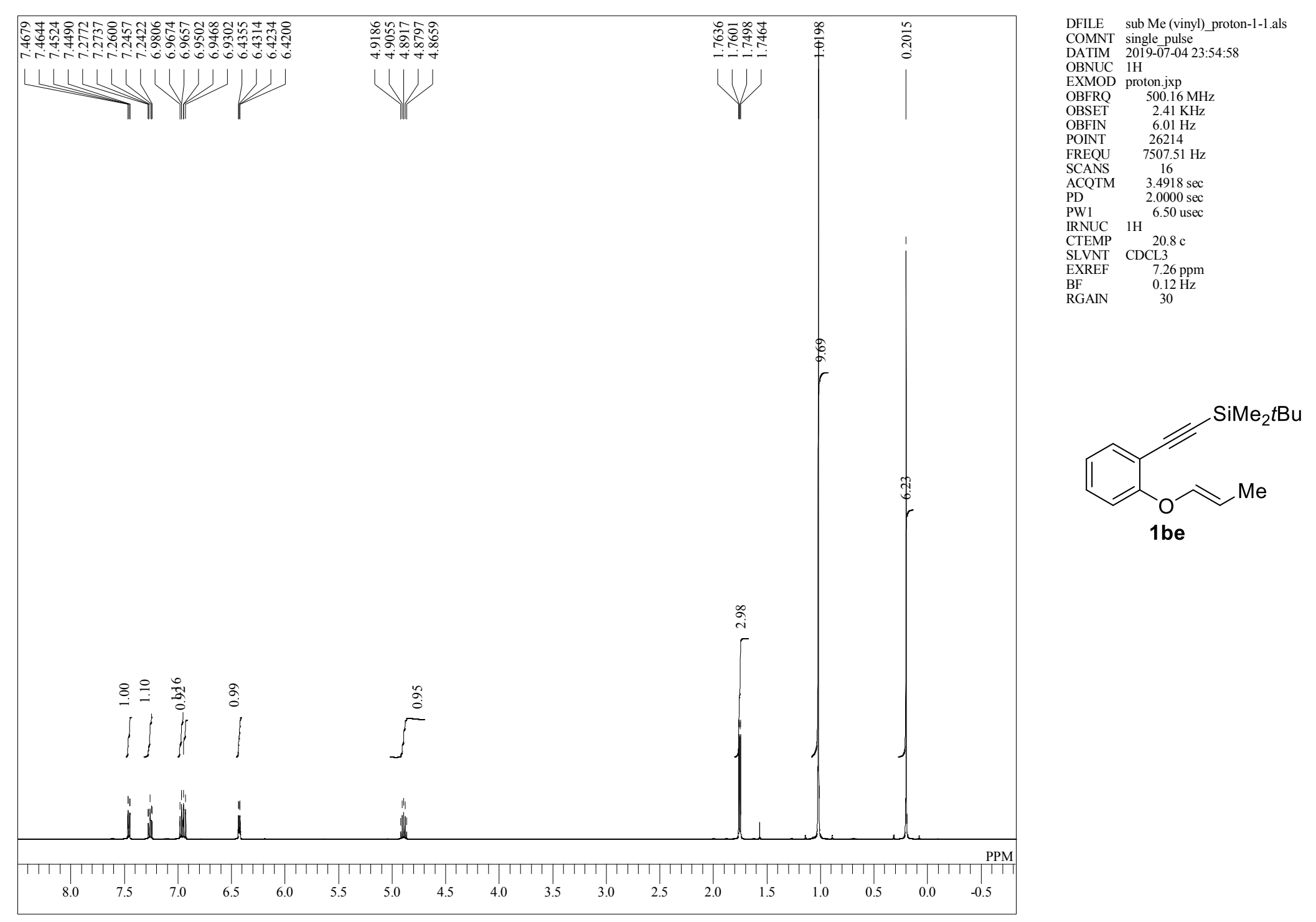


single pulse decoupled gated NOE

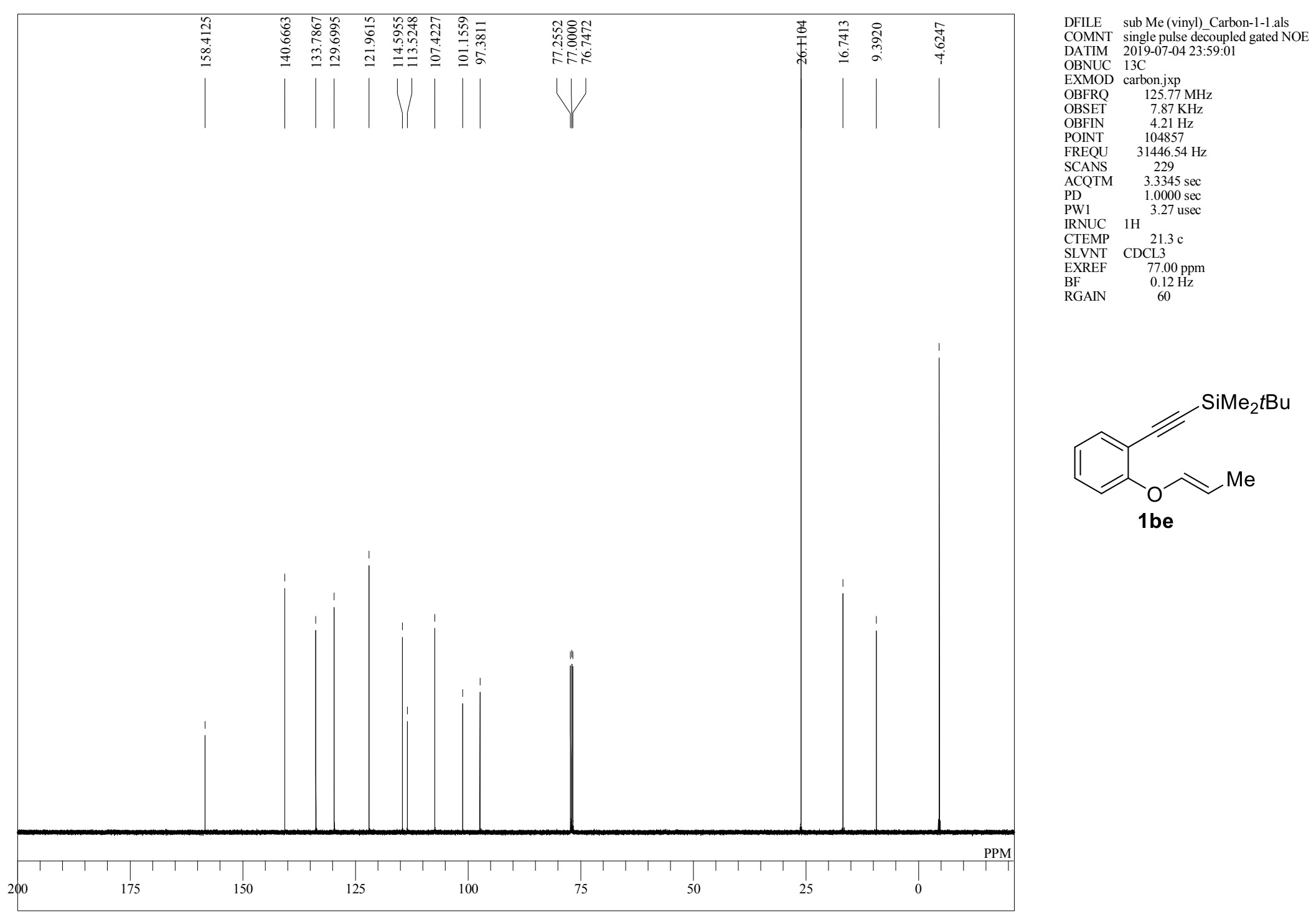


single puls e

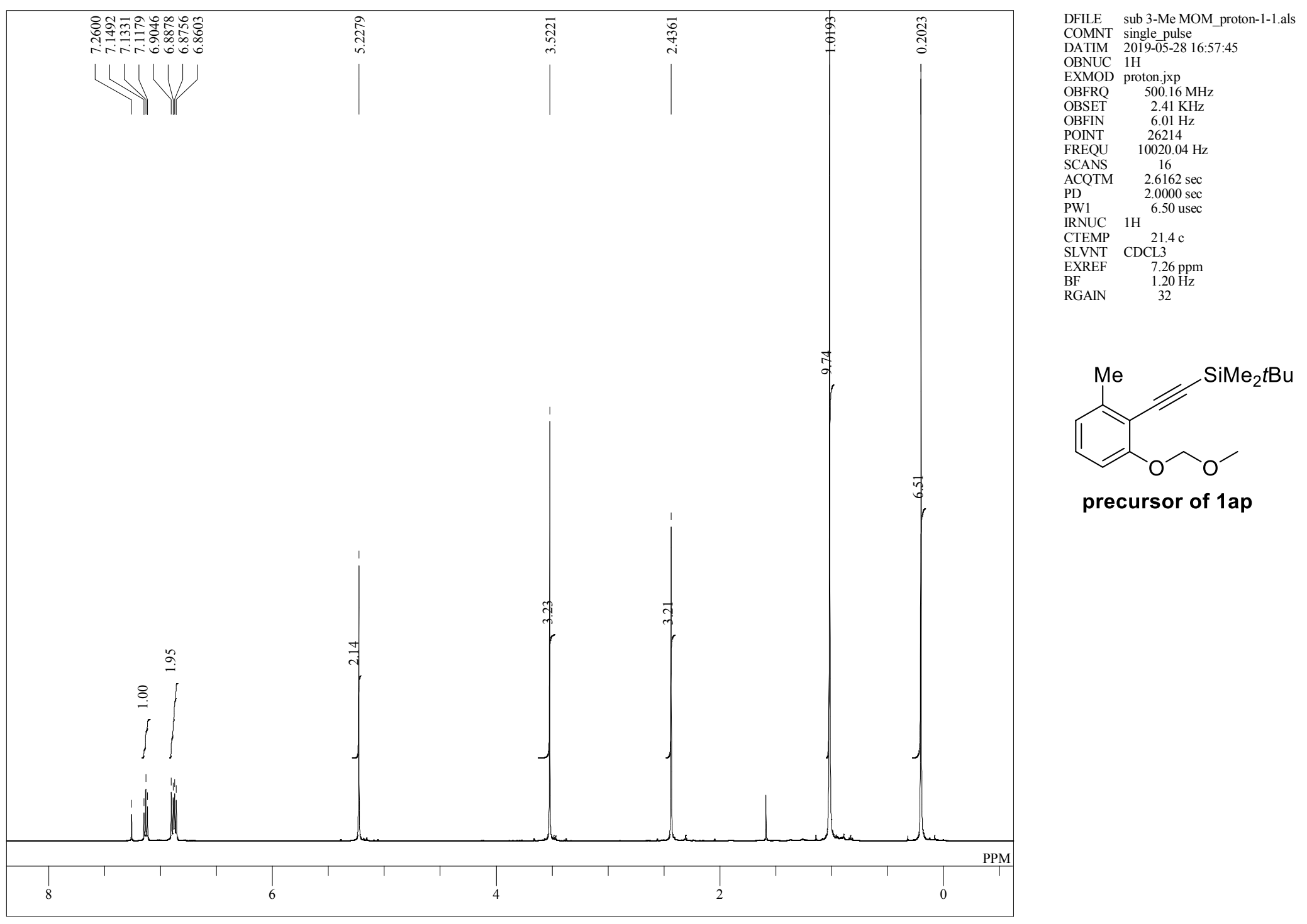

$\mathrm{S} 2-44$ 
single pulse decoupled gated NOE

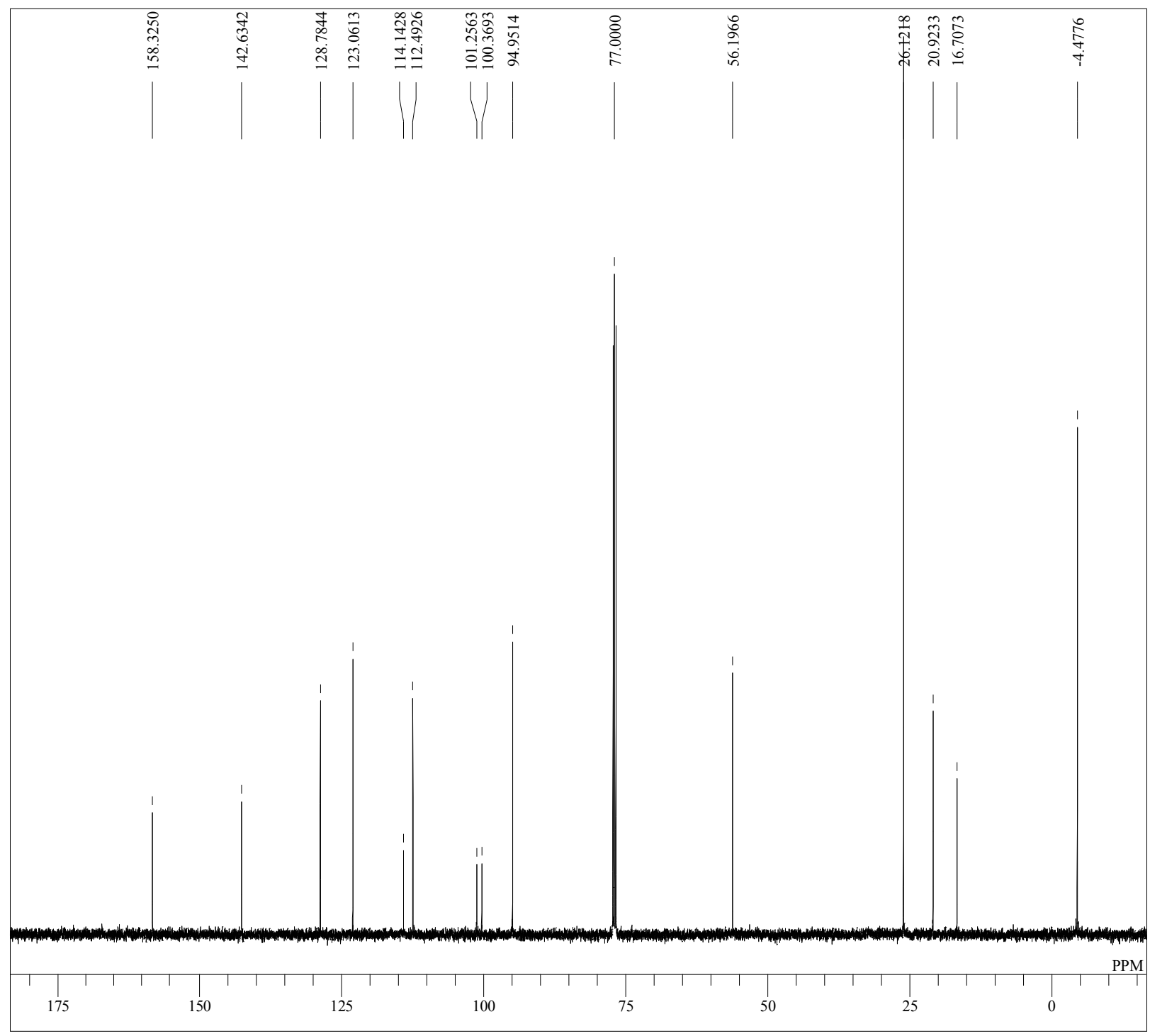

DFILE sub 3-Me MOM_Carbon-1-1.als

DATIM single pulse decoupled gated $\mathrm{NOE}$

OBNUC 13C

$\begin{array}{ll}\text { EXMOD } & \text { carbon.jxp } \\ \text { OBFRQ } & 125.77 \mathrm{MHz}\end{array}$

$\begin{array}{ll}\text { OBFRQ } & 125.77 \mathrm{MHz} \\ \text { OBSET } & 7.87 \mathrm{KHz}\end{array}$

$\begin{array}{ll}\text { OBFIN } & 4.21 \mathrm{KH} \\ \text { POINT } & 26214\end{array}$

FREQU $\quad 31446.54 \mathrm{H} z$

SCANS 121

$\begin{array}{ll}\text { ACQTM } & 0.8336 \mathrm{sec} \\ \mathrm{PD} & 20000 \mathrm{sec}\end{array}$

$\begin{array}{ll}\text { PW1 } & 2.0000 \mathrm{sec} \\ \text { PWN } & 3.27 \mathrm{usec}\end{array}$

IRNUC $1 \mathrm{H}$

$\begin{array}{ll}\text { CTEMP } & 21.6 \mathrm{c} \\ \text { SLVNT } & \text { CDCL3 }\end{array}$

EXREF $\quad 77.00 \mathrm{ppm}$

$\begin{array}{ll}\text { BF } & 1.20 \mathrm{~Hz} \\ \text { RGAIN } & 58\end{array}$

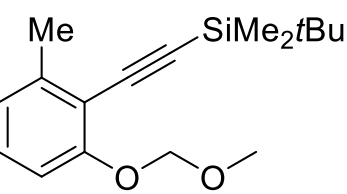

precursor of 1ap 
single pulse

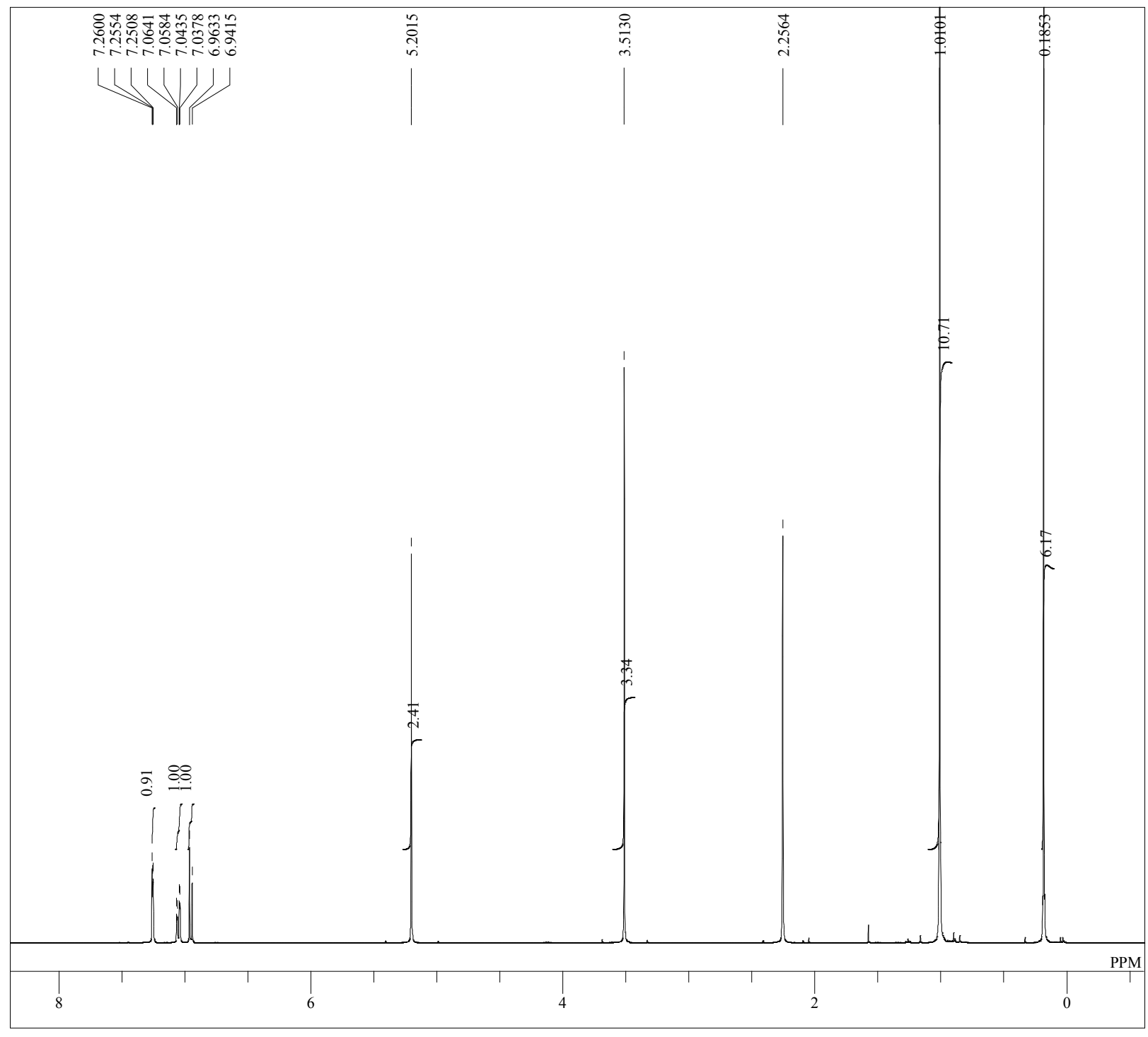

DFILE sub 4-Me MOM_proton-1-1.als

$\begin{array}{ll}\text { COMNT } & \text { single-pulse } \\ \text { DATIM } & \text { 2019-05-29 15:17:37 }\end{array}$

OBNUC $1 \mathrm{H}$

$\begin{array}{ll}\text { EXMOD proton.jxp } \\ \text { OBFRQ } & 399.78 \mathrm{MHz}\end{array}$

OBSET $\quad 4.19 \mathrm{KHz}$

POINT 13107

FREQU $\quad 6002.40 \mathrm{~Hz}$

SCANS 16

PD $\quad 1.0000 \mathrm{sec}$

$\begin{array}{ll}\text { PW1 } & 1.0000 \mathrm{sec} \\ \text { PWN } & 7.25 \mathrm{usec}\end{array}$

IRNUC $1 \mathrm{H}$

$\begin{array}{lr}\text { CTEMP } & 21.5 c^{2} \\ \text { SLVNT } & \text { CDCL3 }\end{array}$

$\begin{array}{ll}\text { EXREF } & 7.26 \mathrm{ppm} \\ \mathrm{BF} & 0.12 \mathrm{~Hz}\end{array}$

RGAIN $\quad 34$

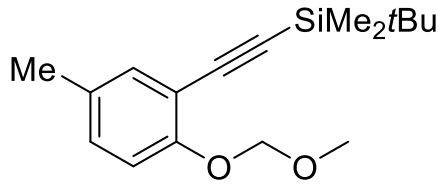

precursor of $1 \mathrm{aq}$ 
single pulse decoupled gated NOE

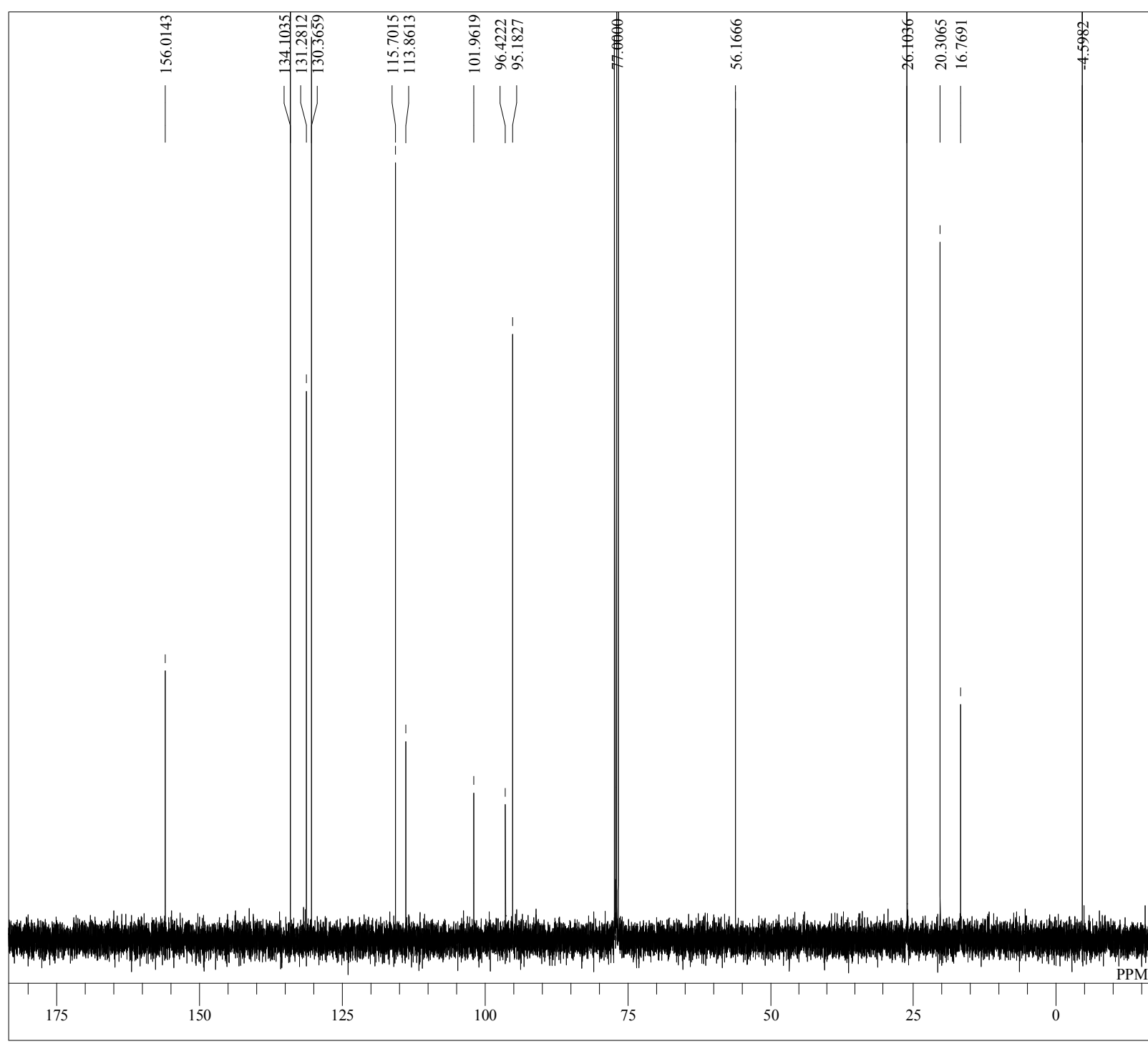

DFILE sub 4-Me MOM_carbon-1-1.als

COMNT single pulse decoupled gated NOE

OBNUC $13 \mathrm{C}$

$\begin{array}{ll}\text { OBFRQ } & \text { carbon.jxp } \\ \text { OBRR } & 100.53 \mathrm{MH} z\end{array}$

OBSET $5.35 \mathrm{KHz}$

$\begin{array}{ll}\text { OBFIN } & 5.86 \mathrm{~Hz} \\ \text { POINT } & 26214\end{array}$

$\begin{array}{lc}\text { FREQU } & 25125.63 \mathrm{~Hz} \\ \text { SCANS } & 219\end{array}$

ACQTM $\quad 1.0433 \mathrm{sec}$

$\begin{array}{ll}\text { PD } & 1.0000 \mathrm{sec} \\ \text { PW1 } & 3.17 \text { usec }\end{array}$

IRNUC $1 \mathrm{H}$

CTEMP $21.7 \mathrm{c}$

EXREF $\quad 77.00 \mathrm{ppm}$

$\begin{array}{lc}\text { BF } & 77.00 \mathrm{ppm} \\ \text { RGAIN } & 60\end{array}$

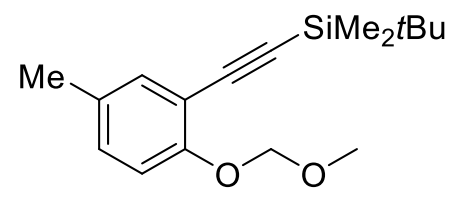

precursor of $1 \mathrm{aq}$ 
single pulse

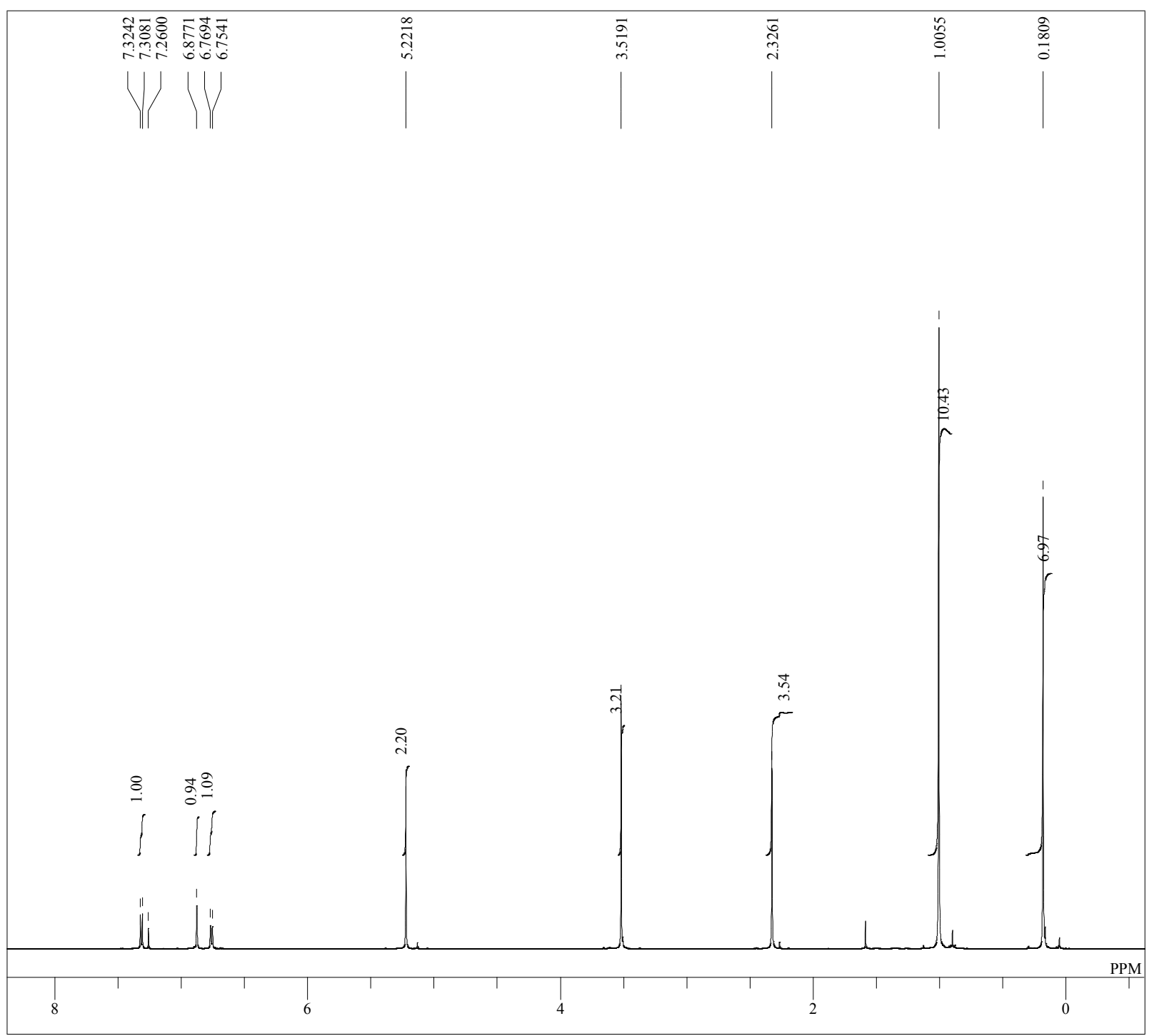

DFILE sub 5-Me MOM proton-1-1.als

DATIM 2019-05-28 17:41:3

OBMODQ
OBroton.jxp
$500.16 \mathrm{MHz}$

OBSET $\quad 2.41 \mathrm{KHz}$

POINT $\quad 6.01 \mathrm{~Hz}$

FREQU $\quad 10020.04 \mathrm{H} z$

$\begin{array}{lc}\text { SCANS } & 16 \\ \text { ACQTM } & 2.6162 \mathrm{sec}\end{array}$

$\begin{array}{ll}\text { ACQTM } & 2.6162 \mathrm{sec} \\ \text { PD } & 2.0000 \mathrm{sec}\end{array}$

PWN 1 1 6.50 usec

CTEMP $21.3 \mathrm{c}$

EXREF $7.26 \mathrm{ppm}$

$\begin{array}{ll}\text { BF } & 1.20 \mathrm{ppm} \\ \text { RGAIN } & 30\end{array}$

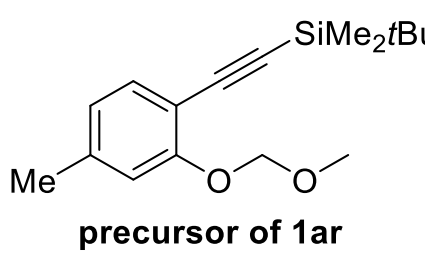

precursor of $1 \mathrm{ar}$ 
single pulse decoupled gated NOE

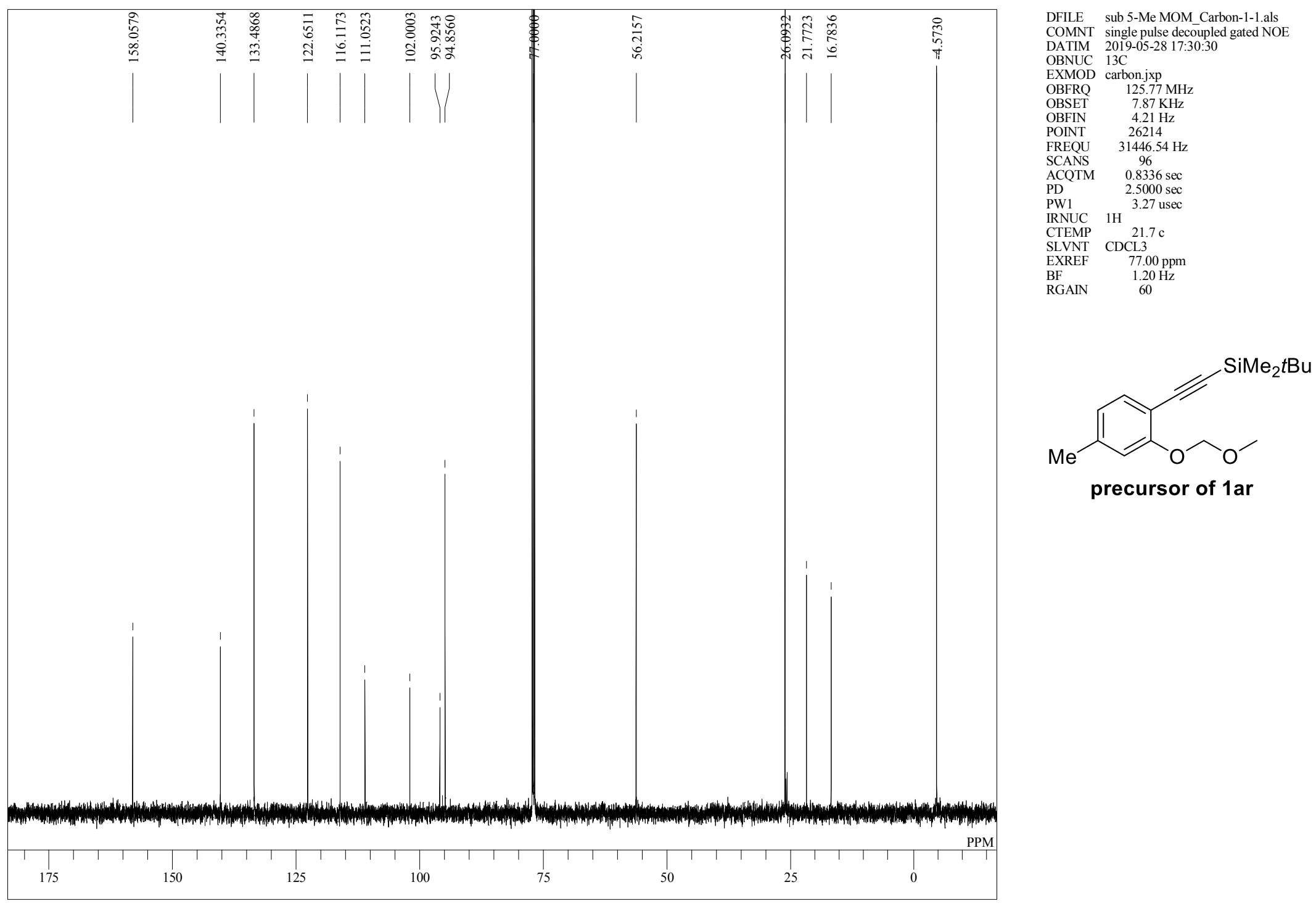

S2 - 49 
single pulse

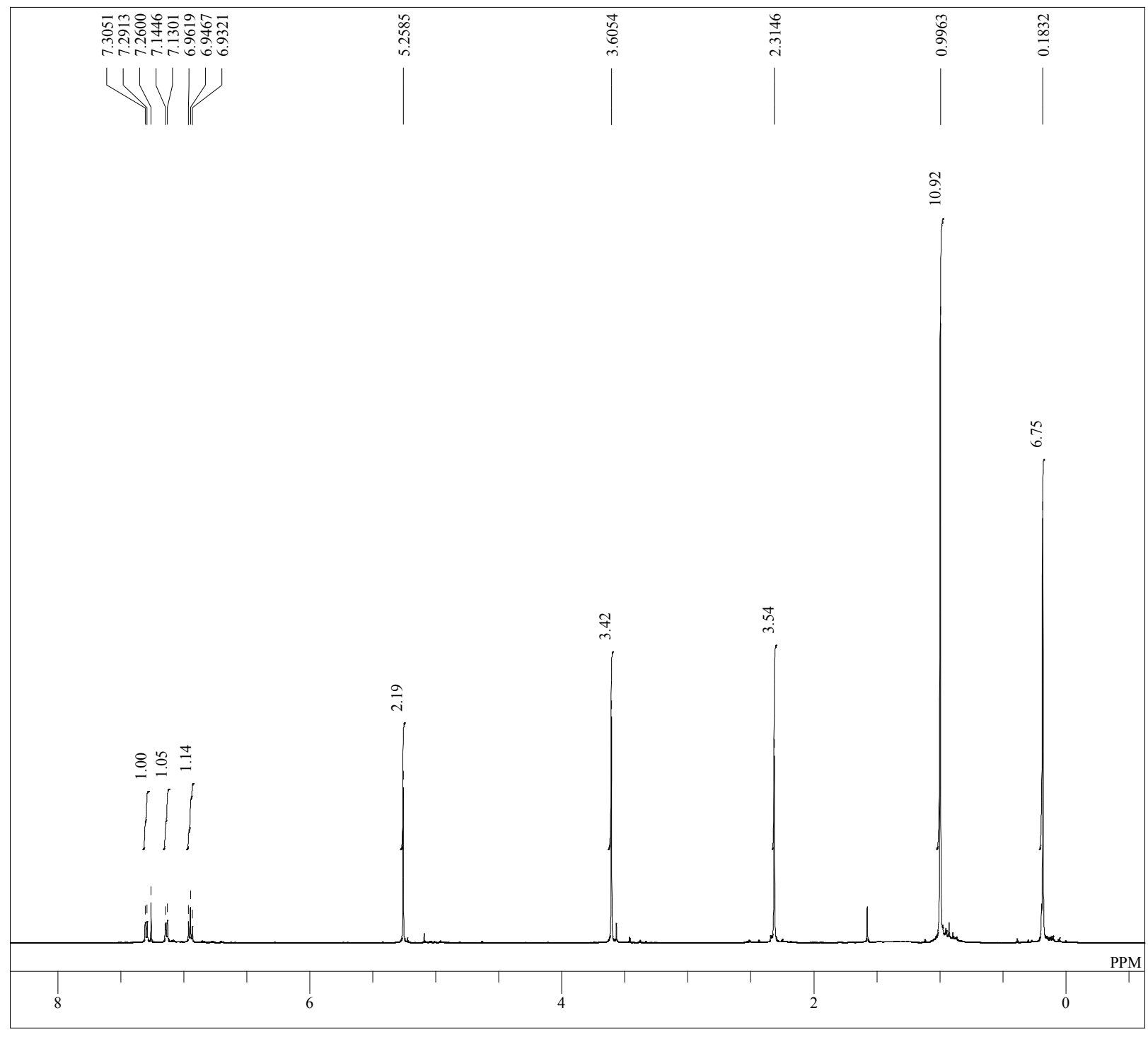

DFILE sub 6-Me MOM_proton-1-1.als

DATM 2019-05-28 17:58:09

OBNUC $1 \mathrm{H}$

$\begin{array}{ll}\text { EXMOD } & \text { proton.jxp } \\ \text { OBFRQ } & 500.16 \mathrm{MHz}\end{array}$

OBSET $\quad 2.41 \mathrm{KH} z$

POINT 26214

$\begin{array}{ll}\text { FREQU } & 10020.04 \mathrm{~Hz}\end{array}$

$\begin{array}{ll}\text { SCANS } & 16 \\ \text { ACOTM } & 2.6162 \mathrm{sec}\end{array}$

$\begin{array}{ll}\mathrm{PD} & 2.6162 \mathrm{sec} \\ & 20000 \mathrm{sec}\end{array}$

PW1 6.50 usec

$\begin{array}{lll}\text { IRNUC } & 1 \mathrm{H} & 6.50 \\ \text { CTEMP } & & 21.5 \mathrm{c}\end{array}$

$\begin{array}{ll}\text { CTEMP } & 21.5 \mathrm{c} \\ \text { SLVNT CDCL3 } & \end{array}$

$\begin{array}{lc}\text { EXREF } & 7.26 \mathrm{ppm} \\ \mathrm{BF} & 1.20 \mathrm{~Hz}\end{array}$

RGAIN
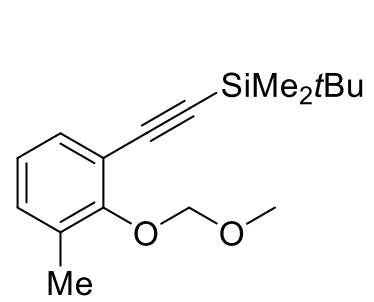

precursor of 1 as 
single pulse decoupled gated NOE

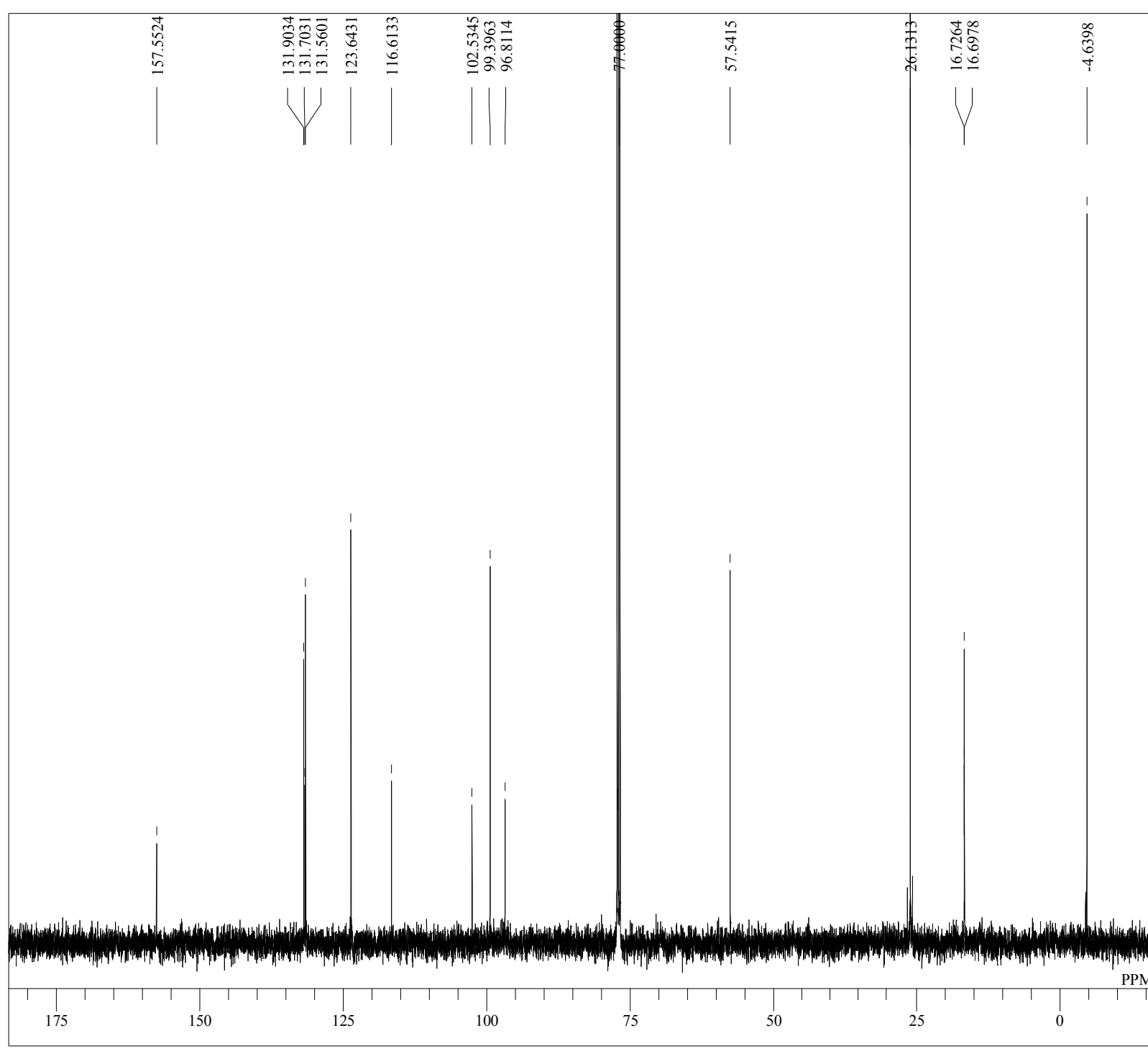

DFILE sub 6-Me MOM_Carbon-1-1.als

DATIM single pulse decoupled gated NOE

OBNUC $13 \mathrm{C}$

OBFRQ $\quad 125.77 \mathrm{MH} z$

$\begin{array}{ll}\text { OBSET } & 7.87 \mathrm{KHz} \\ \text { OBFIN } & 421 \mathrm{~Hz}\end{array}$

POINT 26214

$\begin{array}{lc}\text { FREQU } & 31446.54 \mathrm{~Hz} \\ \text { SCANS } & 89\end{array}$

ACQTM $\quad 0.0000 \mathrm{sec}$

$\begin{array}{ll}\text { PD } & 2.0000 \mathrm{sec} \\ \text { PW1 } & 3.27 \text { usec }\end{array}$

IRNUC $1 \mathrm{H} 21.7 \mathrm{c}$

CTEMP $21.7 \mathrm{c}$

EXREF $\quad 77.00 \mathrm{ppm}$

$\begin{array}{lc}\text { BF } & 1.20 \mathrm{~Hz} \\ \text { RGAIN } & 60\end{array}$

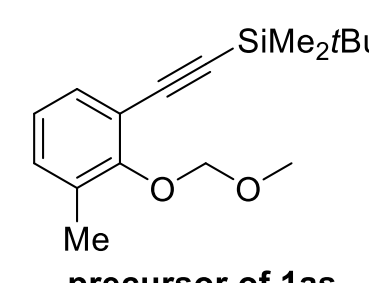

precursor of 1as 
single_pulse

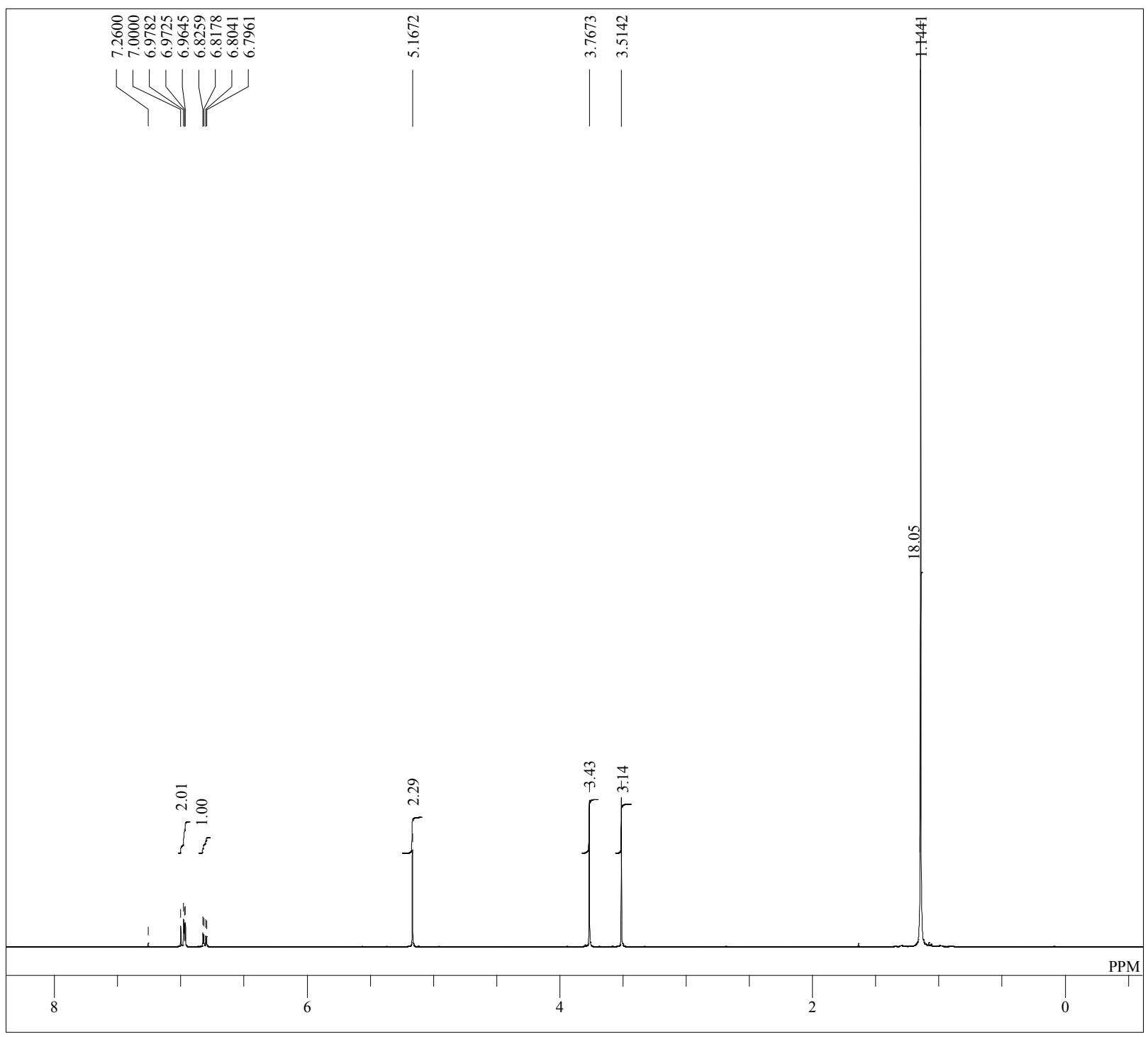

DFILE sub 4-OMe MOM_proton-1-1.als

DATIM single pulse

OBNUC $1 \mathrm{H}$

$\begin{array}{ll}\text { EXMOD } & \text { proton.jxp } \\ \text { OBFRQ } & 399.78 \mathrm{MHz}\end{array}$

OBSET $\quad 4.19 \mathrm{KH} z$

POINT $\quad 13107$

$\begin{array}{ll}\text { FREQU } & 6002.40 \mathrm{~Hz} \\ \text { SCANS } & 16\end{array}$

$\begin{array}{ll}\text { SCANS } & 16 \\ \text { ACOTM } & 2.1837 \mathrm{sec}\end{array}$

$\begin{array}{ll}\text { ACQTM } & 2.1837 \mathrm{sec} \\ \mathrm{PD} & 1.0000 \mathrm{sec}\end{array}$

\begin{tabular}{ll} 
PW1 & $1.0000 \mathrm{sec}$ \\
\hline & $7.25 \mathrm{usec}$
\end{tabular}

IRNUC $1 \mathrm{H} 219 \mathrm{c}$

$\begin{array}{lr}\text { CTEMP } & 21.9 \\ \text { SLVNT } & \end{array}$

$\begin{array}{ll}\text { EXREF } & 7.26 \mathrm{ppm} \\ \mathrm{BF} & 0.12 \mathrm{~Hz}\end{array}$

RGAIN 24

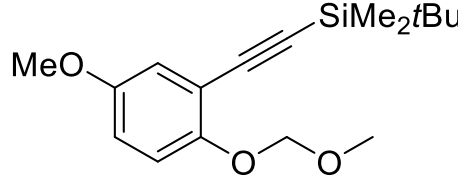

precursor of 1at 
single pulse decoupled gated NOE

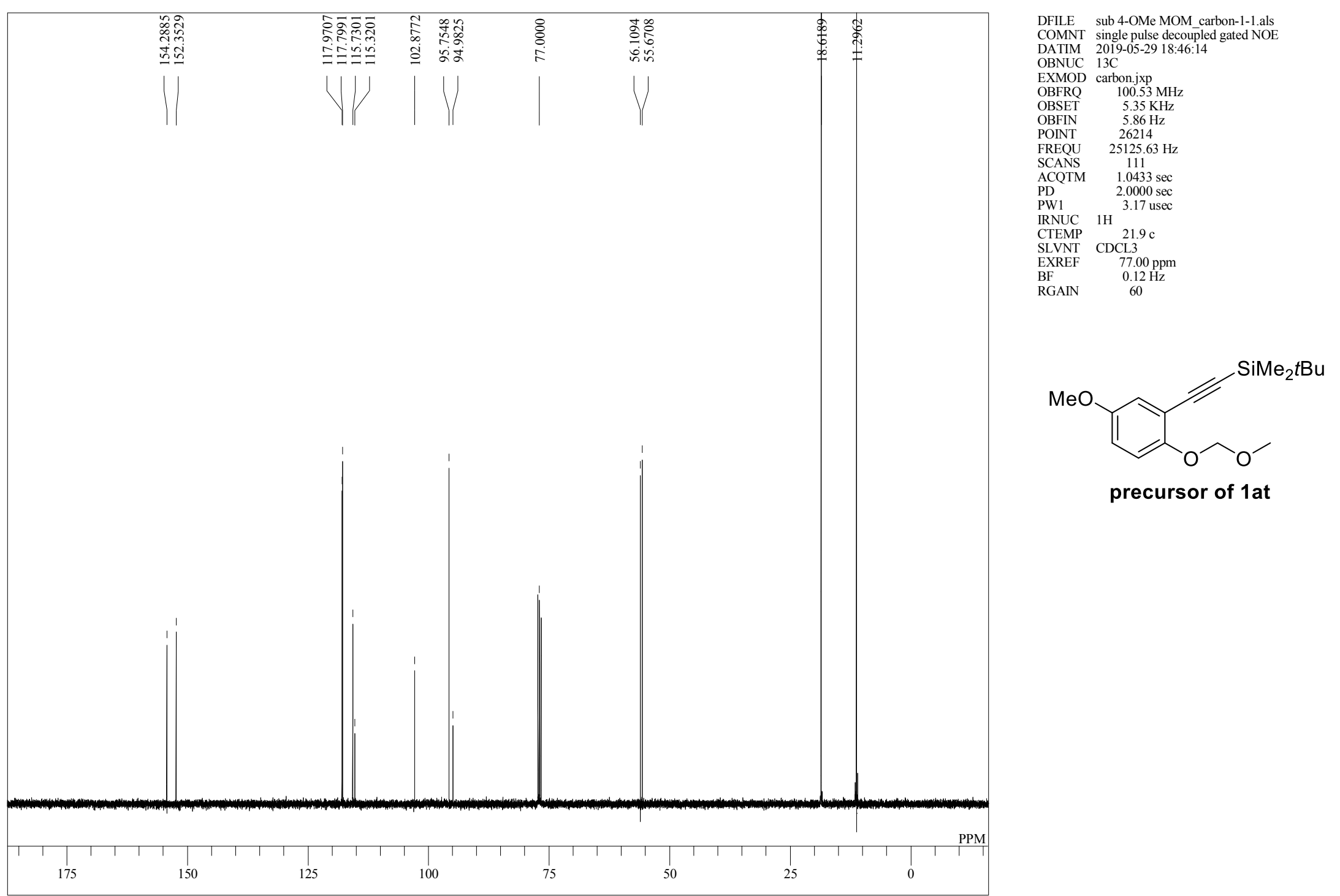


single _pulse

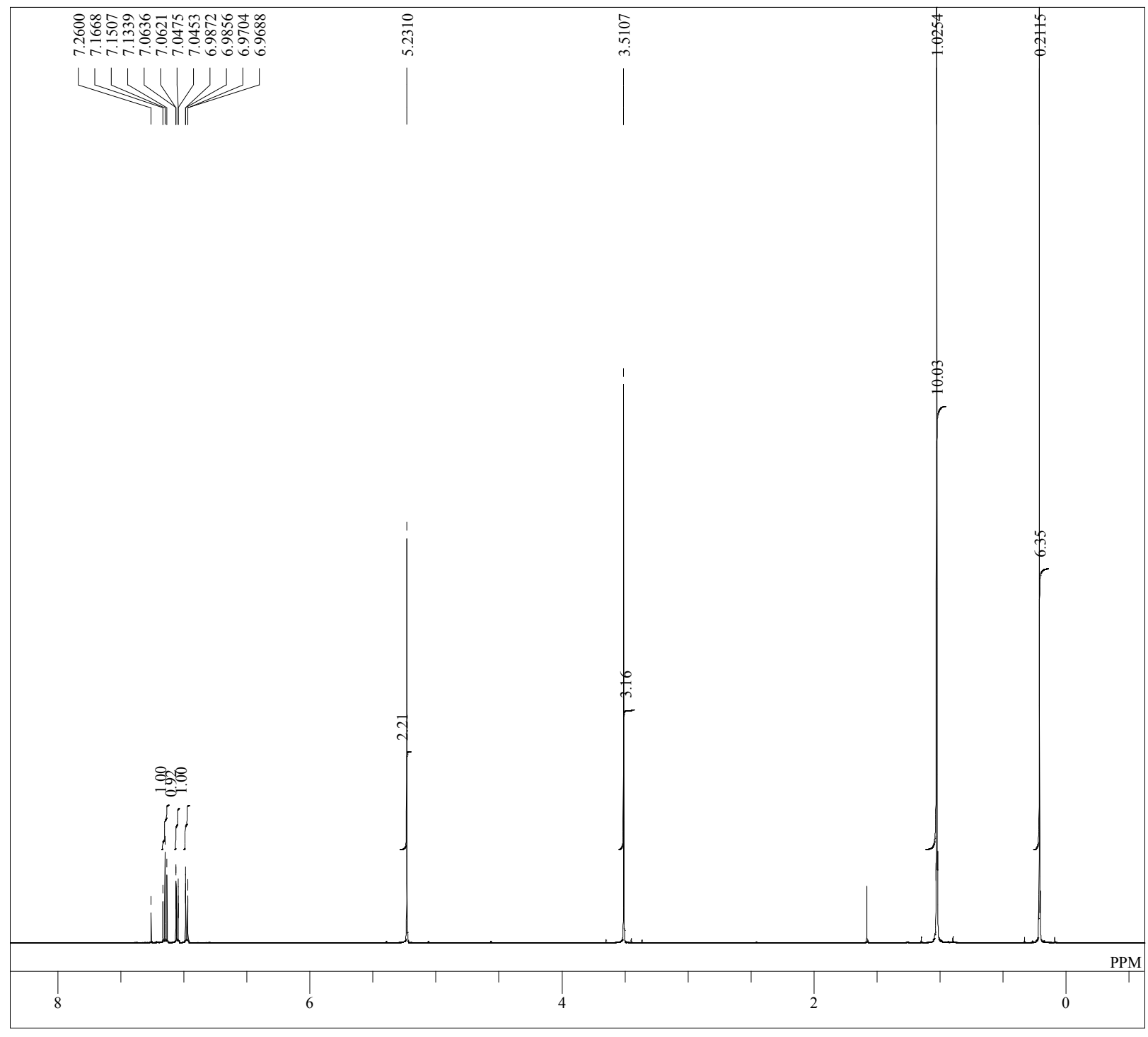

DFILE sub 3-Cl MOM_proton-1-1.als

DATIM 2019-05 -24 $11 \cdot 24,4$

OBNUC $1 \mathrm{H}$

EXMOD p

OBFRQ $\quad 500.16 \mathrm{MHz}$

OBSET $\quad 2.41 \mathrm{KHz}$

POINT 26214

$\begin{array}{ll}\text { FREQU } & 10020.04 \mathrm{~Hz}\end{array}$

$\begin{array}{ll}\text { SCANS } & 16 \\ \text { ACOTM } & 2.6162 \mathrm{sec}\end{array}$

PD $\quad 2.6162 \mathrm{sec}$

PW1 6.50 usec

IRNUC $1 \mathrm{H}$

$\begin{array}{lr}\text { CTEMP } & 20.2 \\ \text { SLVNT } & \text { CDCL3 }\end{array}$

$\begin{array}{ll}\text { EXREF } & 7.26 \mathrm{ppm} \\ \mathrm{BF} & 0.12 \mathrm{~Hz}\end{array}$

RGAIN 34

$\mathrm{Cl} \mathrm{SiMe}_{2} t \mathrm{Bu}$

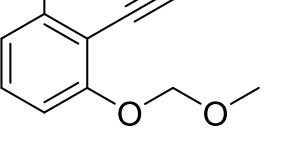

precursor of $1 \mathrm{au}$ 
single pulse decoupled gated NOE

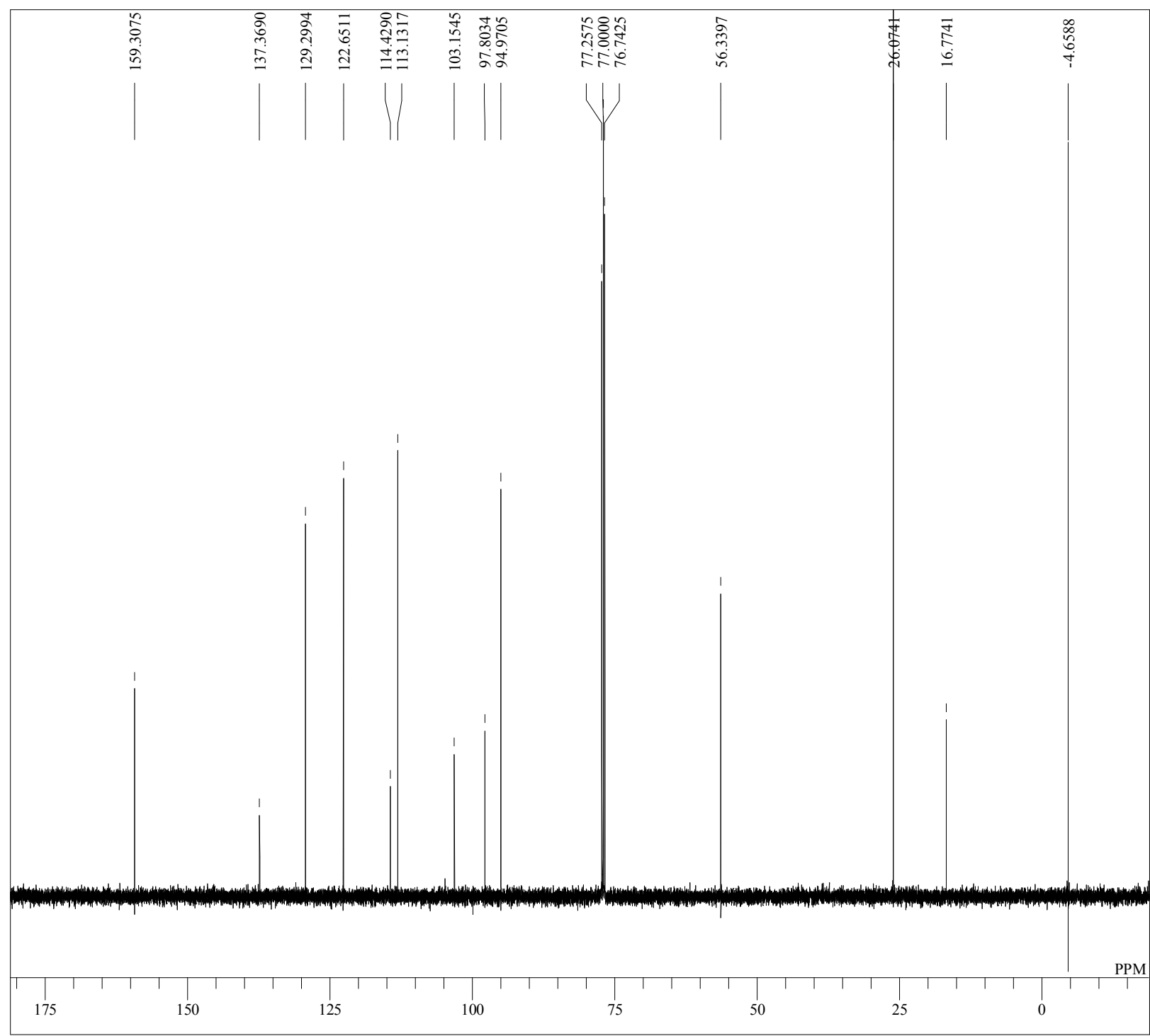

DFILE sub 3-Cl MOM_Carbon-1-1.als

作

OBNUC $13 \mathrm{C}$

OBFRQ $\quad 125.77 \mathrm{MHz}$

OBSET $\quad 7.87 \mathrm{KH}$

POINT 26214

FREQU $\quad 31446.54 \mathrm{~Hz}$

ACQTM $0.8336 \mathrm{sec}$

$\mathrm{PD} \quad 20000 \mathrm{sec}$

$\begin{array}{lr}\text { PW1 } & 3.27 \text { usec }\end{array}$

$\begin{array}{lll}\text { IRNUC } & 1 \mathrm{H} & \\ \text { CTEMP } & & 20.4 \mathrm{c}\end{array}$

$\begin{array}{ll}\text { CTEMP } & 20.4 \mathrm{c} \\ \text { SLVNT } & \text { CDCL3 }\end{array}$

$\begin{array}{ll}\text { EXREF } & 77.00 \mathrm{ppm} \\ \text { BF } & 0.12 \mathrm{~Hz}\end{array}$

RGAIN

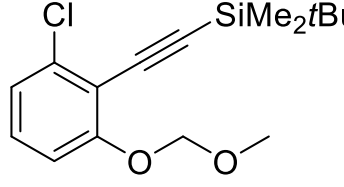

precursor of 1au 
single pulse

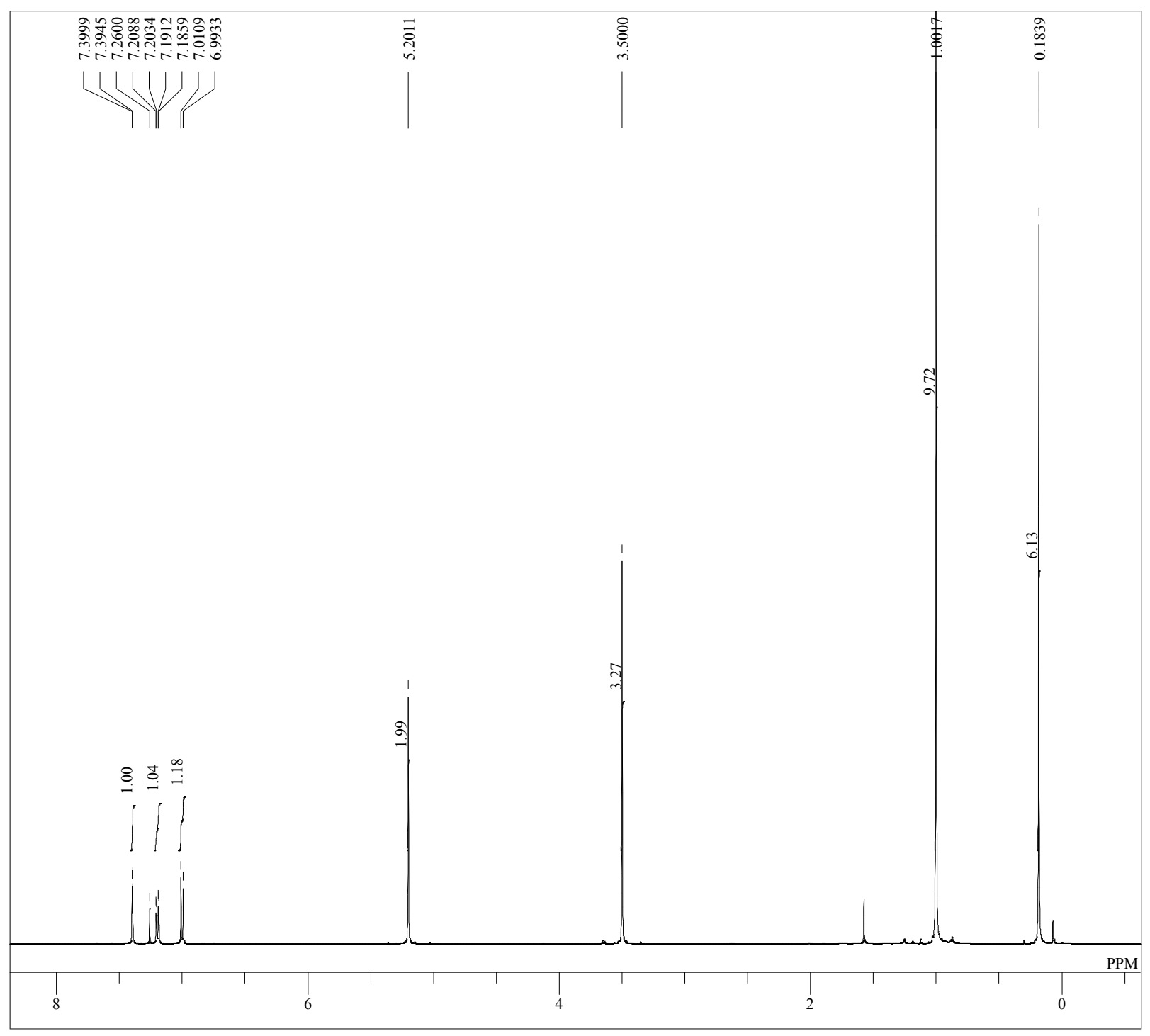

DFILE sub 4-Cl MOM_proton-1-1.als

$\begin{array}{ll}\text { COMNT } & \text { single pulse } \\ \text { DATIM } & 2019-05-28 \text { 12:21:17 }\end{array}$

EXMOD proton.jxp

$\begin{array}{ll}\text { OBFRQ } & 500.16 \mathrm{MHz} \\ \text { OBSET } & 2.41 \mathrm{KHz}\end{array}$

$6.01 \mathrm{~Hz}$

FREQU $\quad 10020.04 \mathrm{~Hz}$

SCANS 16

$\begin{array}{ll}\mathrm{PD} & 2.6162 \mathrm{sec} \\ \mathrm{P} & 20000 \mathrm{sec}\end{array}$

$\begin{array}{ll}\text { PD } & 2.0000 \mathrm{sec} \\ \text { PW1 } & 6.50 \mathrm{usec}\end{array}$

$\begin{array}{lll}\text { IRNUC } & 1 \mathrm{H} & \\ \text { CTEMP } & 21.0 \mathrm{c}\end{array}$

SLVNT CDCL3 $21.0 \mathrm{c}$

$\begin{array}{ll}\text { EXREF } & 7.26 \mathrm{ppm} \\ \mathrm{BF} & 1.20 \mathrm{~Hz}\end{array}$

RGAIN

36

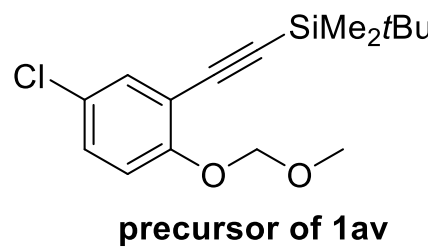

S2 - 56 
single pulse decoupled gated NOE

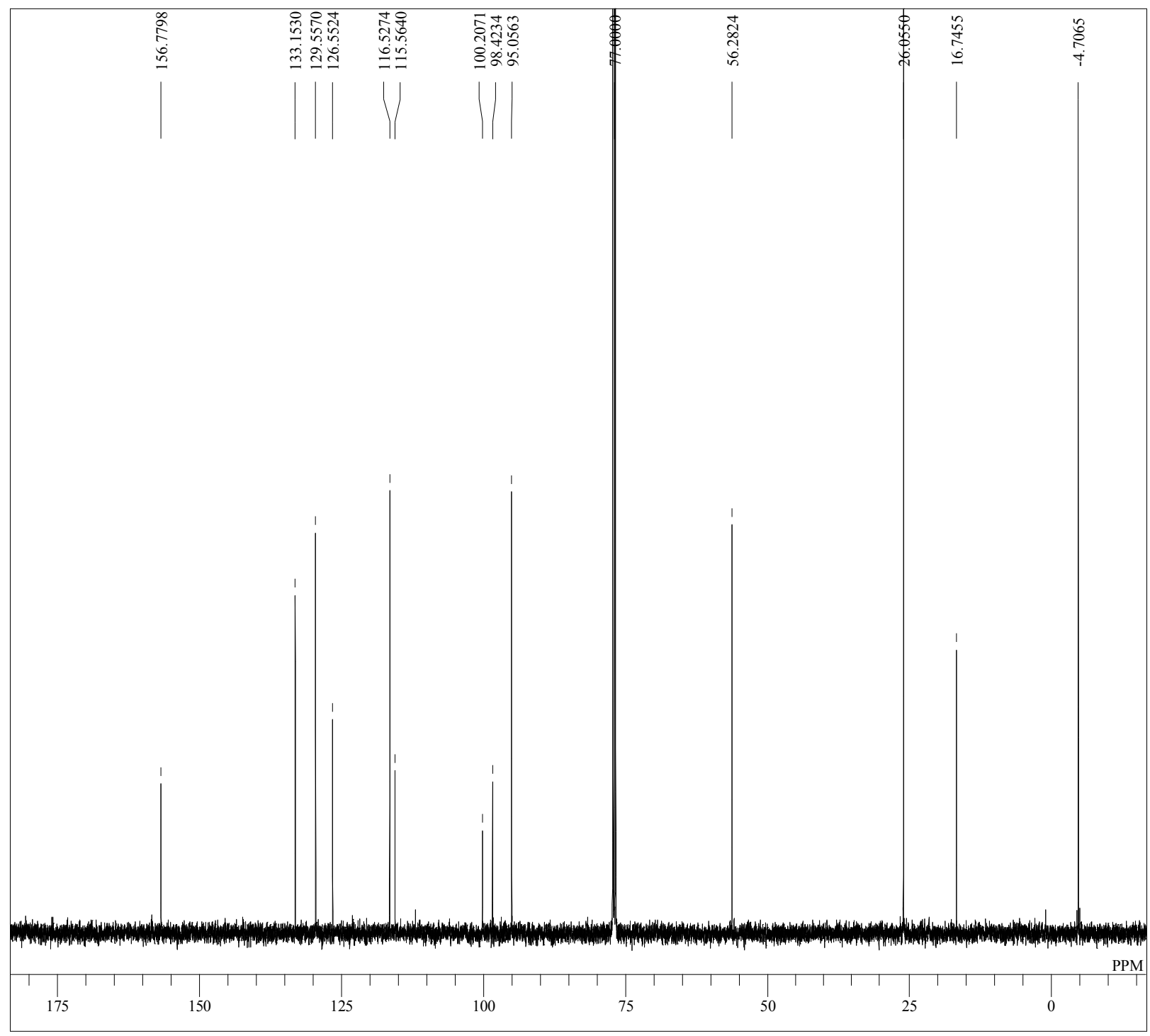

DFILE sub 4-Cl MOM_Carbon-1-1.als

COMNT single pulse decoupled gated NOE

OBNUC $13 \mathrm{C}$

EXMOD carbon.jxp
OBFRO $125.77 \mathrm{MH}$

$\begin{array}{ll}\text { OBFRQ } & 125.77 \mathrm{MHz} \\ \text { OBSET } & 7.87 \mathrm{KHz}\end{array}$

$\begin{array}{ll}\text { OBSET } & 4.21 \mathrm{Kz} \\ \text { OBIN } & 26214\end{array}$

FREQU $\quad 31446.54 \mathrm{~Hz}$

$\begin{array}{ll}\text { SCANS } & 152 \\ \text { ACQTM } & 0.8336 \mathrm{sec}\end{array}$

$\begin{array}{ll}\text { ACQTM } & 0.8336 \mathrm{sec} \\ \mathrm{PD} & 20000 \mathrm{sec}\end{array}$

\begin{tabular}{lll} 
PW1 & $2.0000 \mathrm{sec}$ \\
\hline & $3.27 \mathrm{usec}$
\end{tabular}

$\begin{array}{lll}\text { IRNUC } & 1 \mathrm{H} & 21.2 \mathrm{c} \\ \text { CTEMP } & & 21.2 \mathrm{c}\end{array}$

SLVNT CDCL3

$\begin{array}{ll}\text { EXREF } & 77.00 \mathrm{ppm} \\ \mathrm{BF} & 1.20 \mathrm{~Hz}\end{array}$

RGAIN

$.00 \mathrm{ppm}$
$.20 \mathrm{~Hz}$

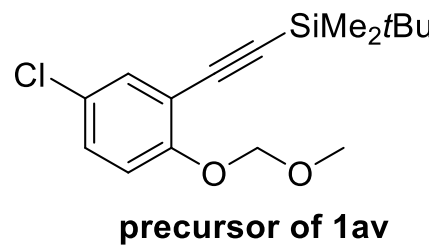


single pulse

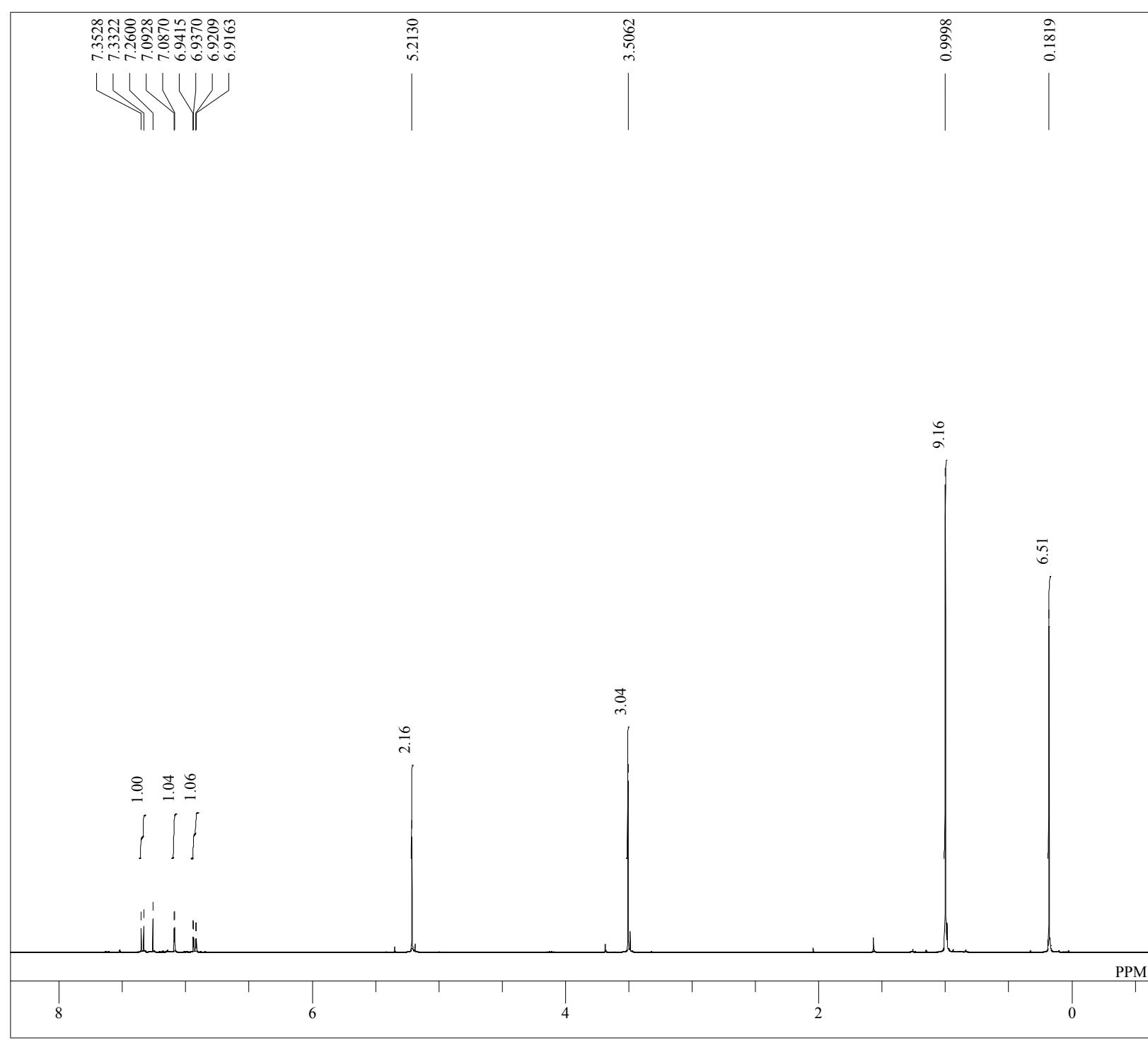

DFILE sub 5-Cl MOM_proton-1-1.als

DATIM 2019-05-24 17:04:07

OBNUC $1 \mathrm{H}$

$\begin{array}{ll}\text { EXMOD } & \text { proton.jxp } \\ \text { OBFRQ } & 399.78 \mathrm{MHz}\end{array}$

$\begin{array}{ll}\text { OBFRQ } & 399.78 \mathrm{MHz} \\ \text { OBSET } & 4.19 \mathrm{KHz}\end{array}$

POINT 13107

FREQU $\quad 6002.40 \mathrm{~Hz}$

$\begin{array}{ll}\text { SCANS } & 16 \\ \text { ACQTM } & 21837\end{array}$

$\begin{array}{ll}\text { ACQTM } & 2.1837 \mathrm{sec} \\ \mathrm{PD} & 1.0000 \mathrm{sec}\end{array}$

$\begin{array}{ll}\text { PW1 } & 7.25 \mathrm{usec}\end{array}$

$\begin{array}{ll}\text { IRNUC } & 1 \mathrm{H} \\ \text { CTEMP } & \\ 21.6 \mathrm{c}\end{array}$

SLVNT CDCL3 3

$\begin{array}{ll}\text { EXREF } & 7.26 \mathrm{ppn} \\ \text { BF } & 0.12 \mathrm{~Hz}\end{array}$

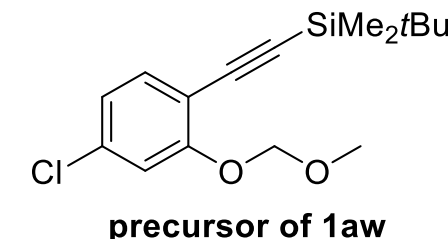


single pulse decoupled gated NOE

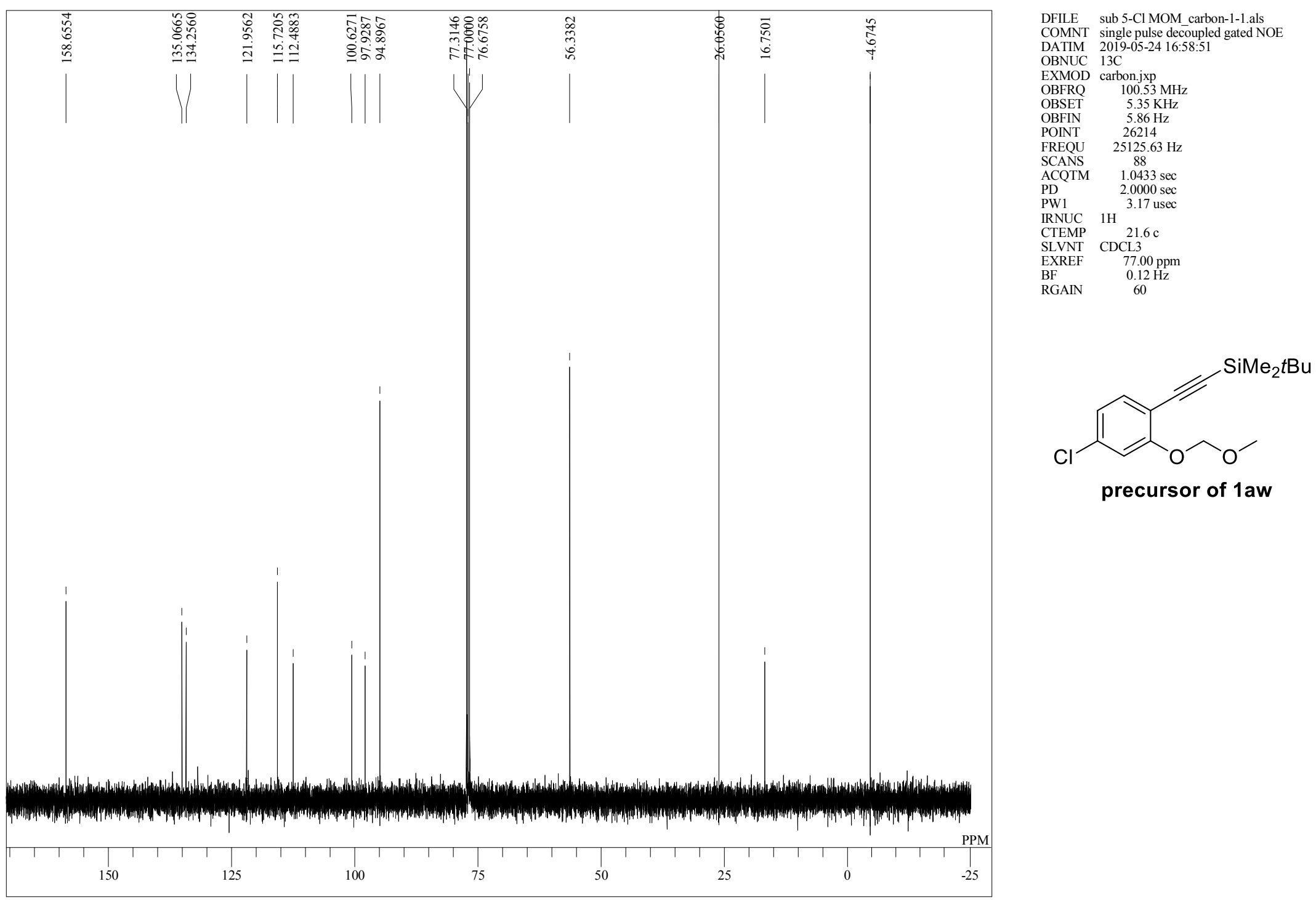


single pulse

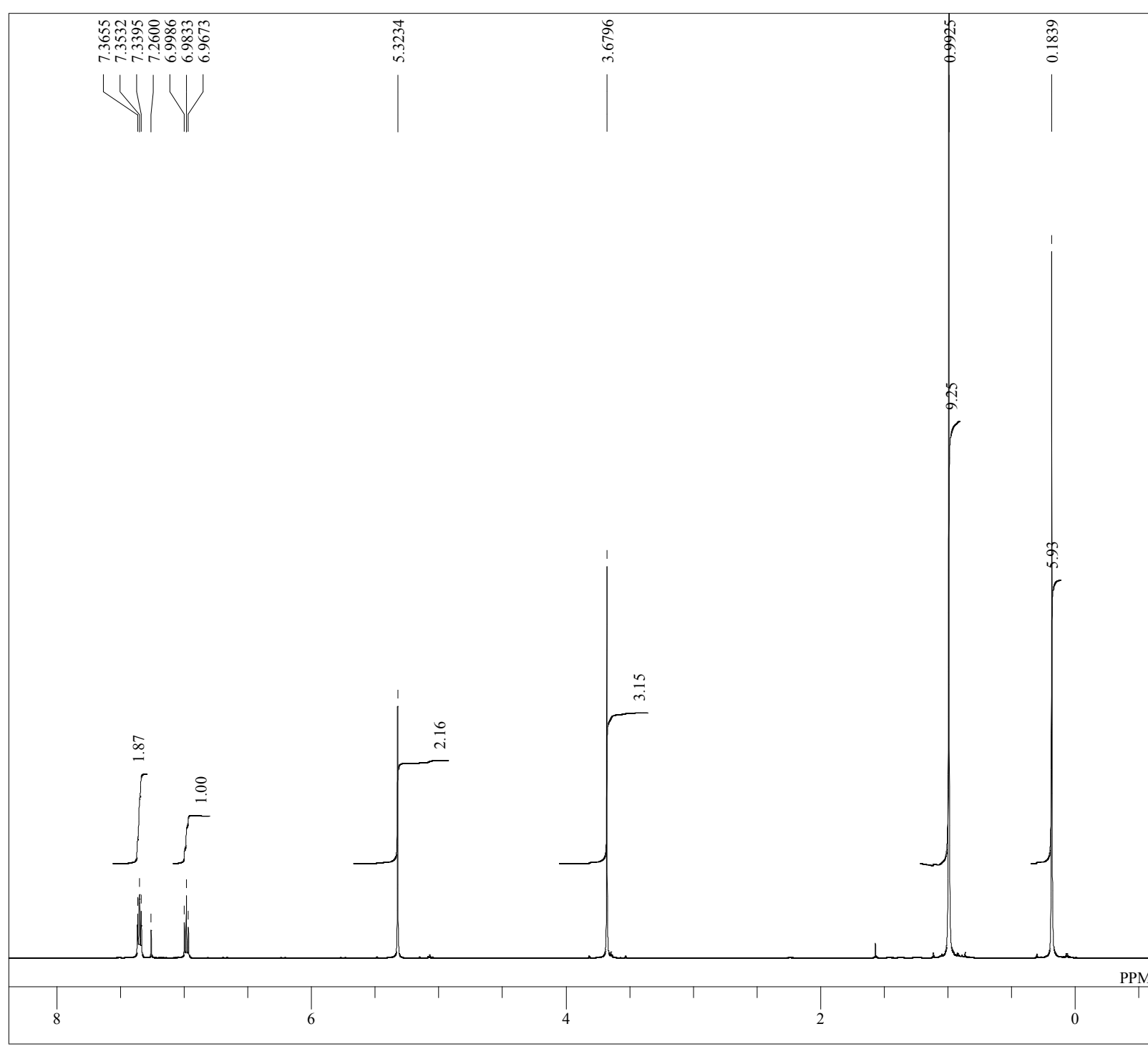

DFILE sub 6-Cl MOM_proton-1-1.als

COMNT single pulse

$\begin{array}{ll}\text { EXMOD proton.jxp } \\ \text { OBFRQ } & 500.16 \mathrm{MHz}\end{array}$

OBSET $\quad 2.41 \mathrm{KHz}$

$\begin{array}{ll}\text { OBFIN } & 6.01 \mathrm{~Hz} \\ \text { POINT } & 26214\end{array}$

FREQU $\quad 10020.04 \mathrm{~Hz}$

SCANS $\quad 16$

$\begin{array}{ll}\text { ACQTM } & 2.6162 \mathrm{sec} \\ \mathrm{PD} & 2.0000 \mathrm{sec} \\ \mathrm{PW} 1 & 6.50 \mathrm{usec}\end{array}$

PW1 $\quad 6.50$ usec

$\begin{array}{lll}\text { IRNUC } & 1 \mathrm{H} & \\ \text { CTEMP } & & 20.0 \mathrm{c}\end{array}$

SLVNT CDCL3

$\begin{array}{ll}\text { EXREF } & 7.26 \mathrm{ppm} \\ \text { BF } & 1.20 \mathrm{~Hz} \\ \text { RGAIN } & 34\end{array}$

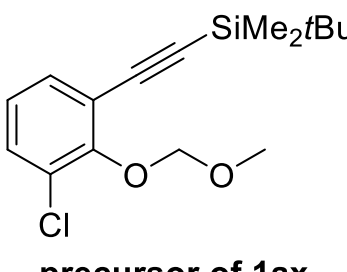

precursor of $1 \mathrm{ax}$ 
single pulse decoupled gated NOE

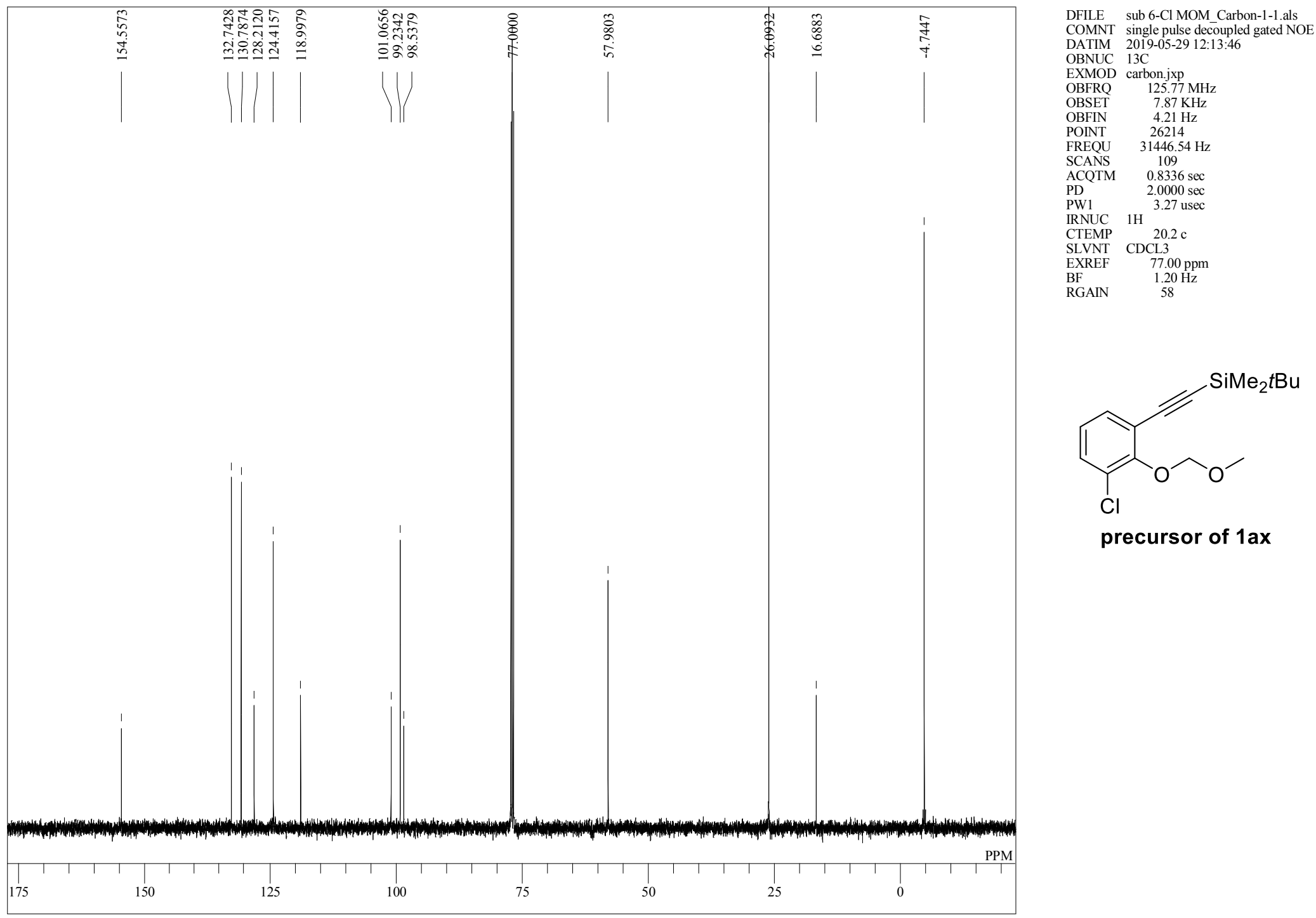


single pulse

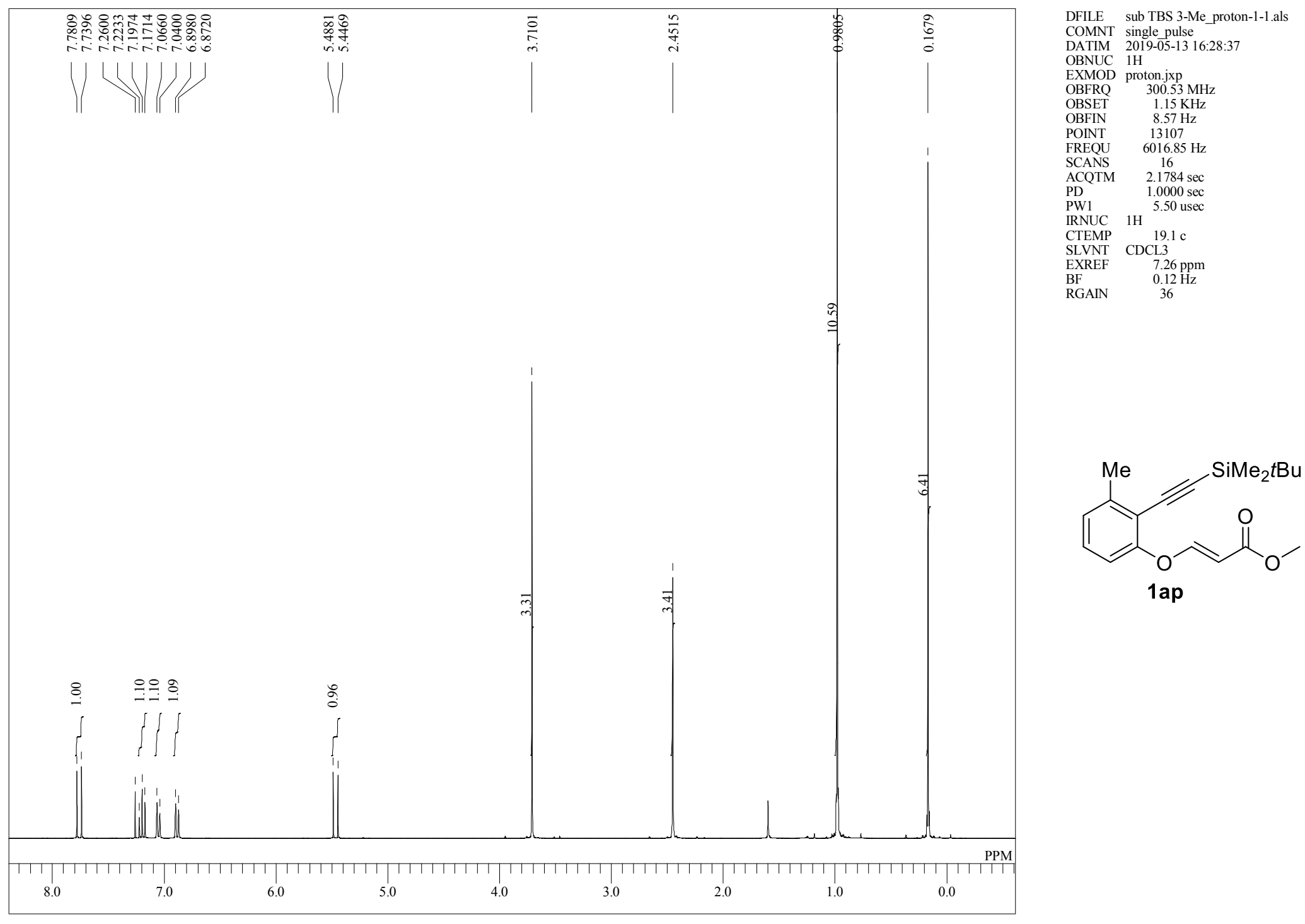

S2 - 62 
single pulse decoupled gated NOE

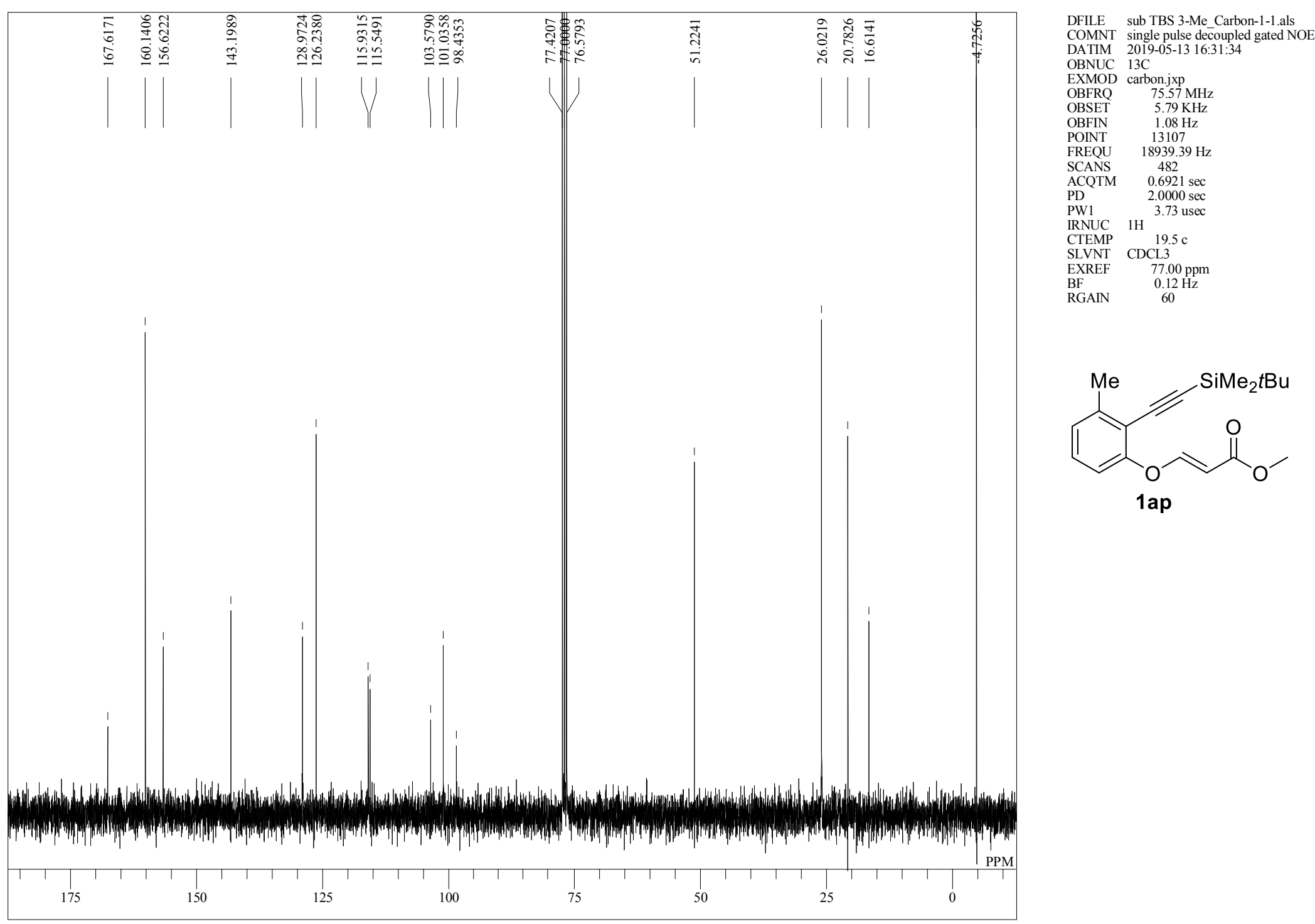

S2 - 63 
single pulse

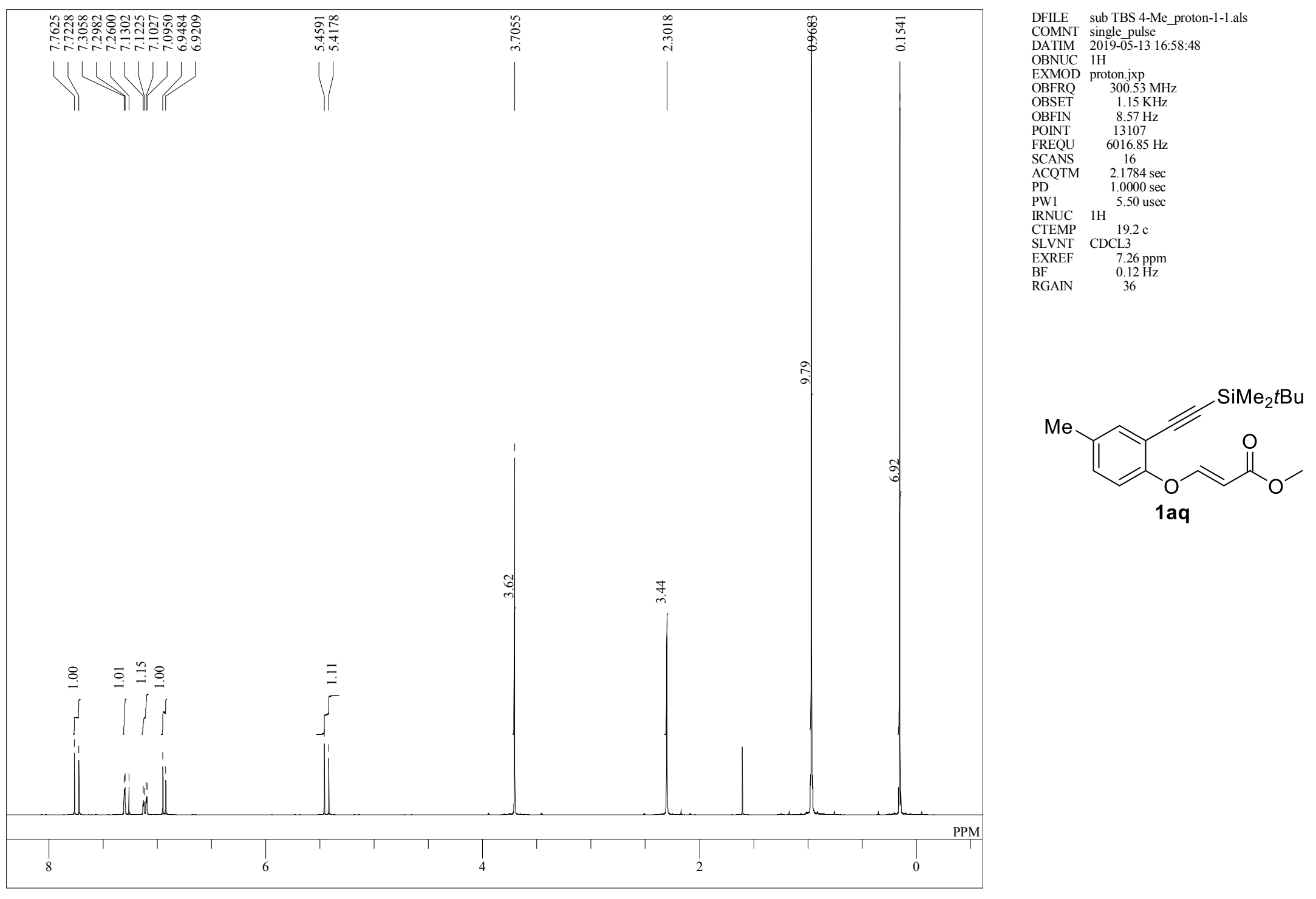


single pulse decoupled gated NOE

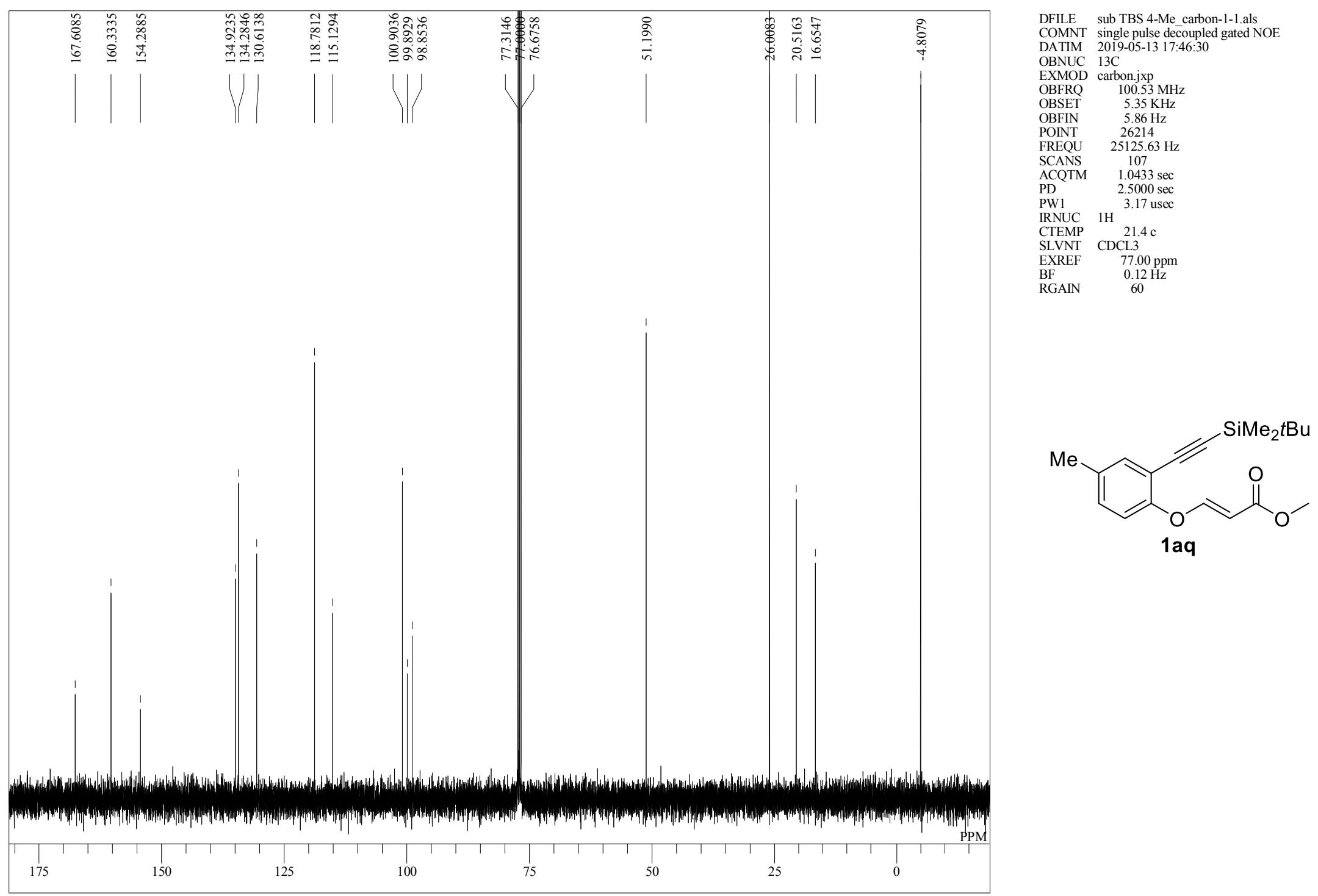


single pulse

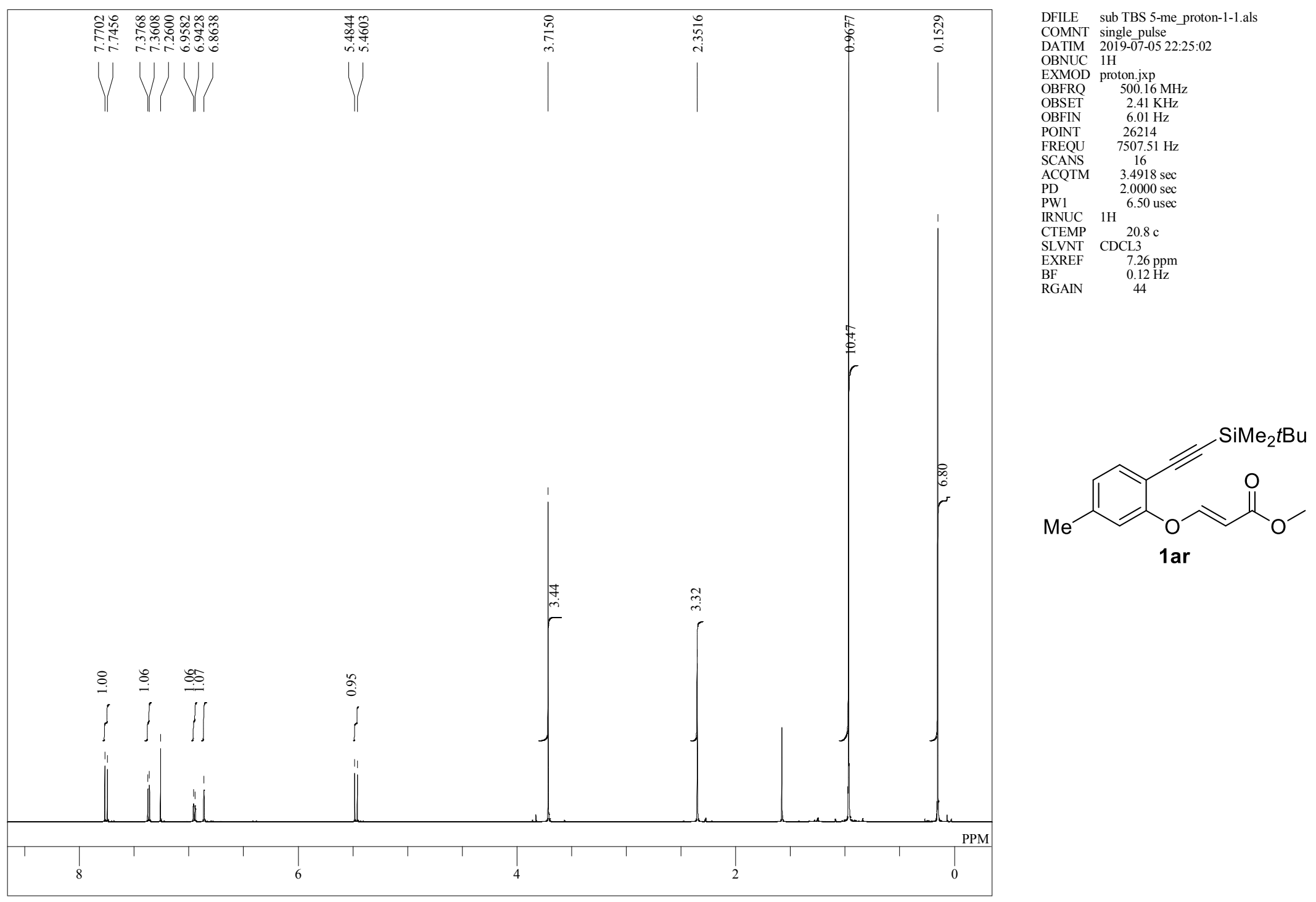


single pulse decoupled gated NOE

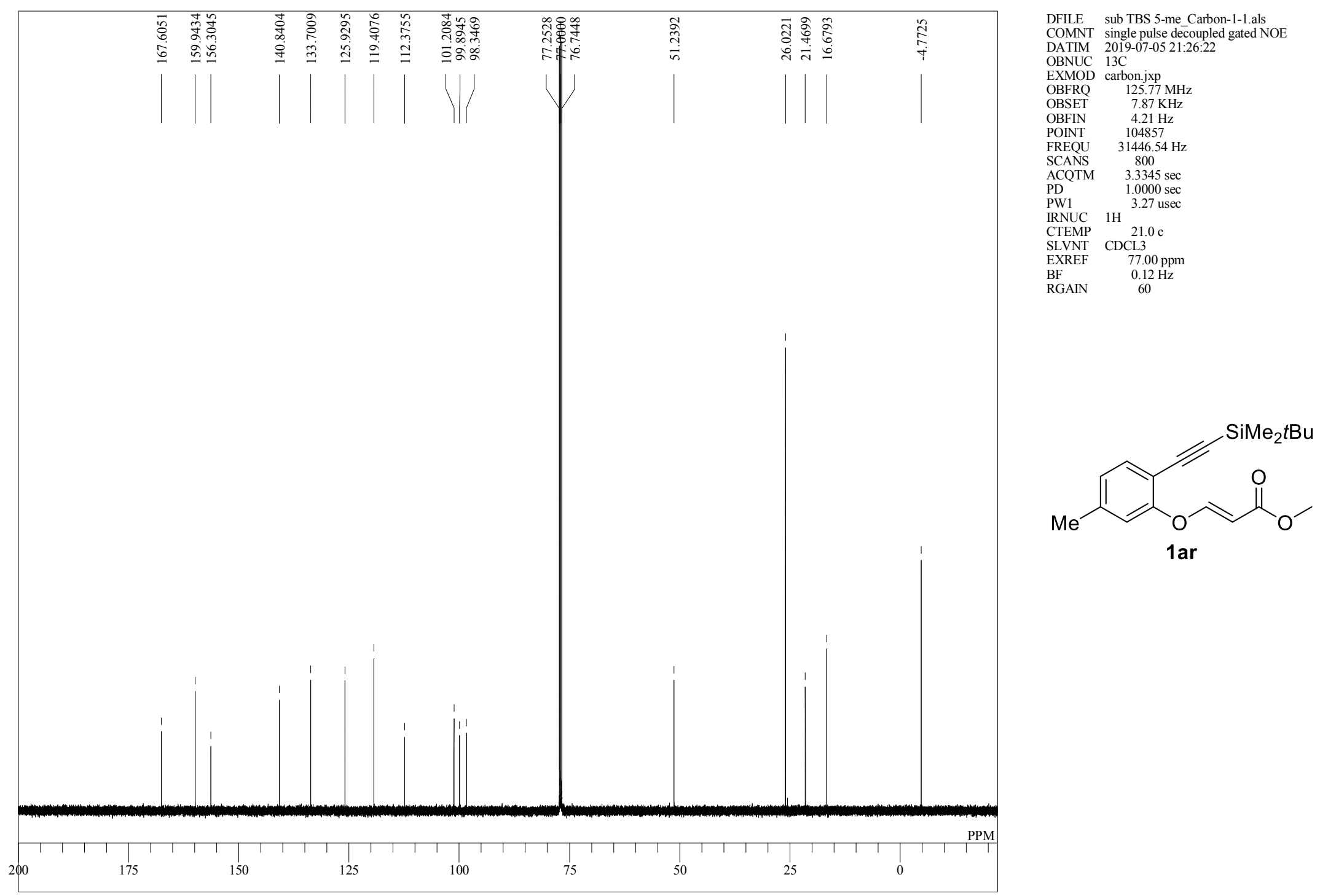


single pulse

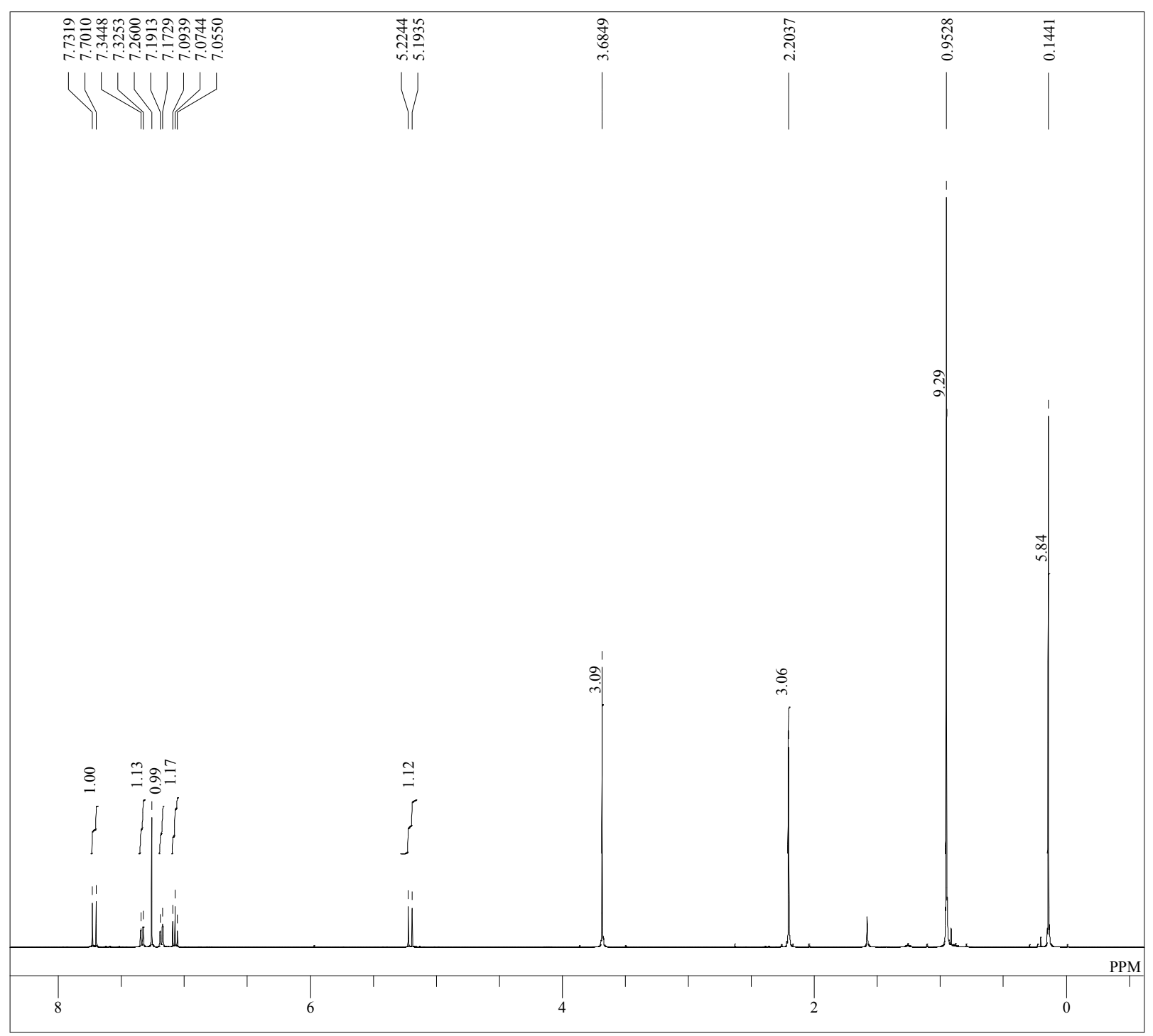

DFILE sub TBS 6-Me_proton-1-1.als

$\begin{array}{ll}\text { DATIM } & \text { single-pulse } \\ \text { 2019-05-13 18:20:19 }\end{array}$

OBFRQ $399.78 \mathrm{MH}$

OBSET $\quad 4.19 \mathrm{KHz}$

POINT $\quad 13107$

FREQU $\quad 6002.40 \mathrm{~Hz}$

SCANS $\quad 16$

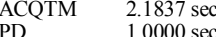

$\begin{array}{ll}\text { PD } & 1.0000 \mathrm{sec} \\ \text { PW1 } & 7.25 \text { usec }\end{array}$

IRNUC 1H

$\begin{array}{ll}\text { CTEMP } & 21.1 \mathrm{c} \\ \text { SLVNT CDCL3 } & \end{array}$

EXREF $7.26 \mathrm{ppm}$

$\begin{array}{ll}\text { BF } & 0.12 \mathrm{~Hz} \\ \text { RGAIN } & 42\end{array}$

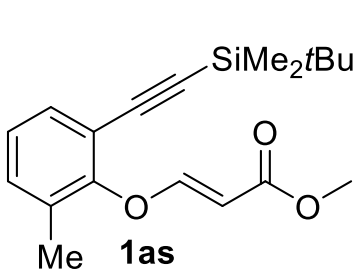


single pulse decoupled gated NOE

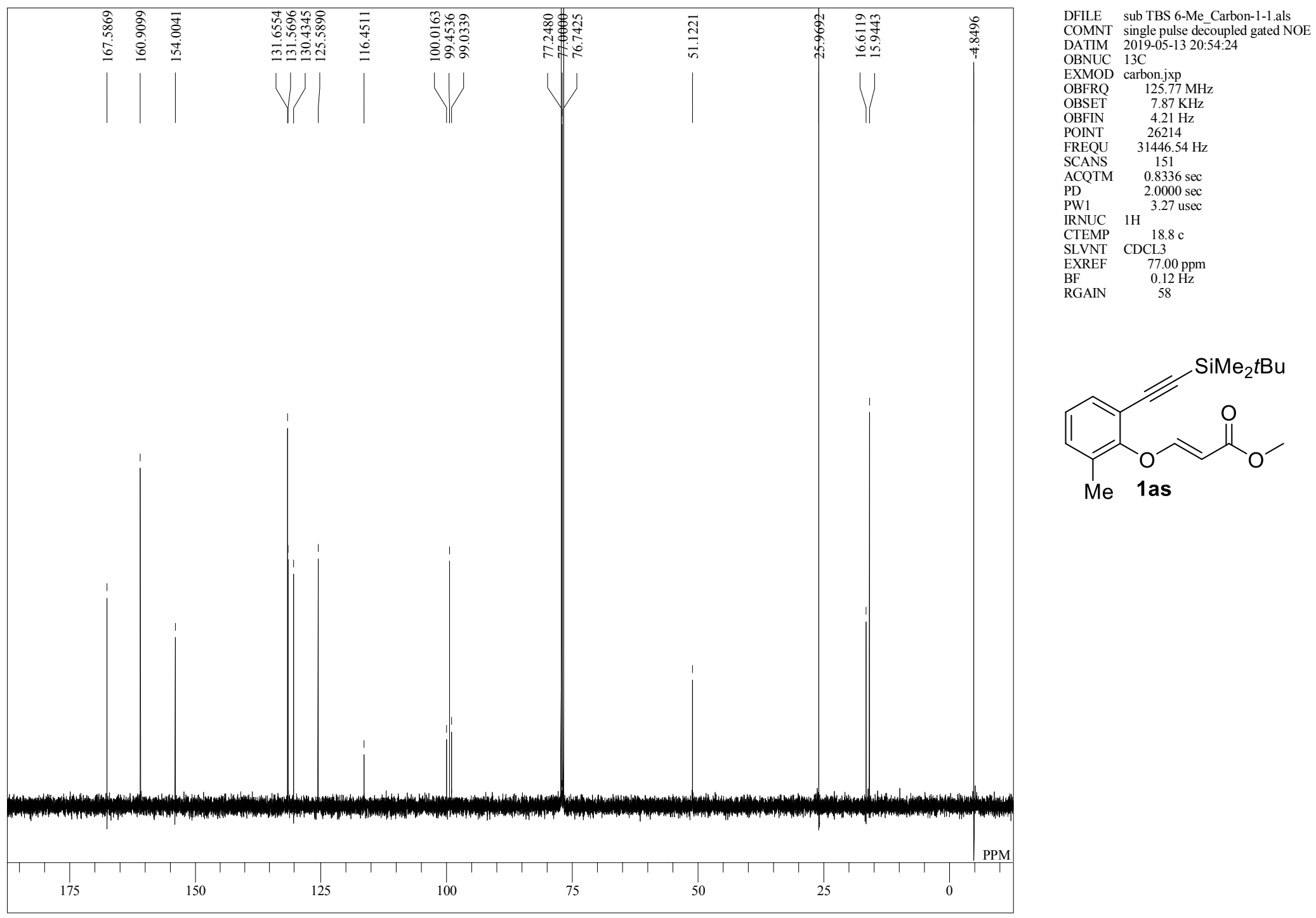


single pulse

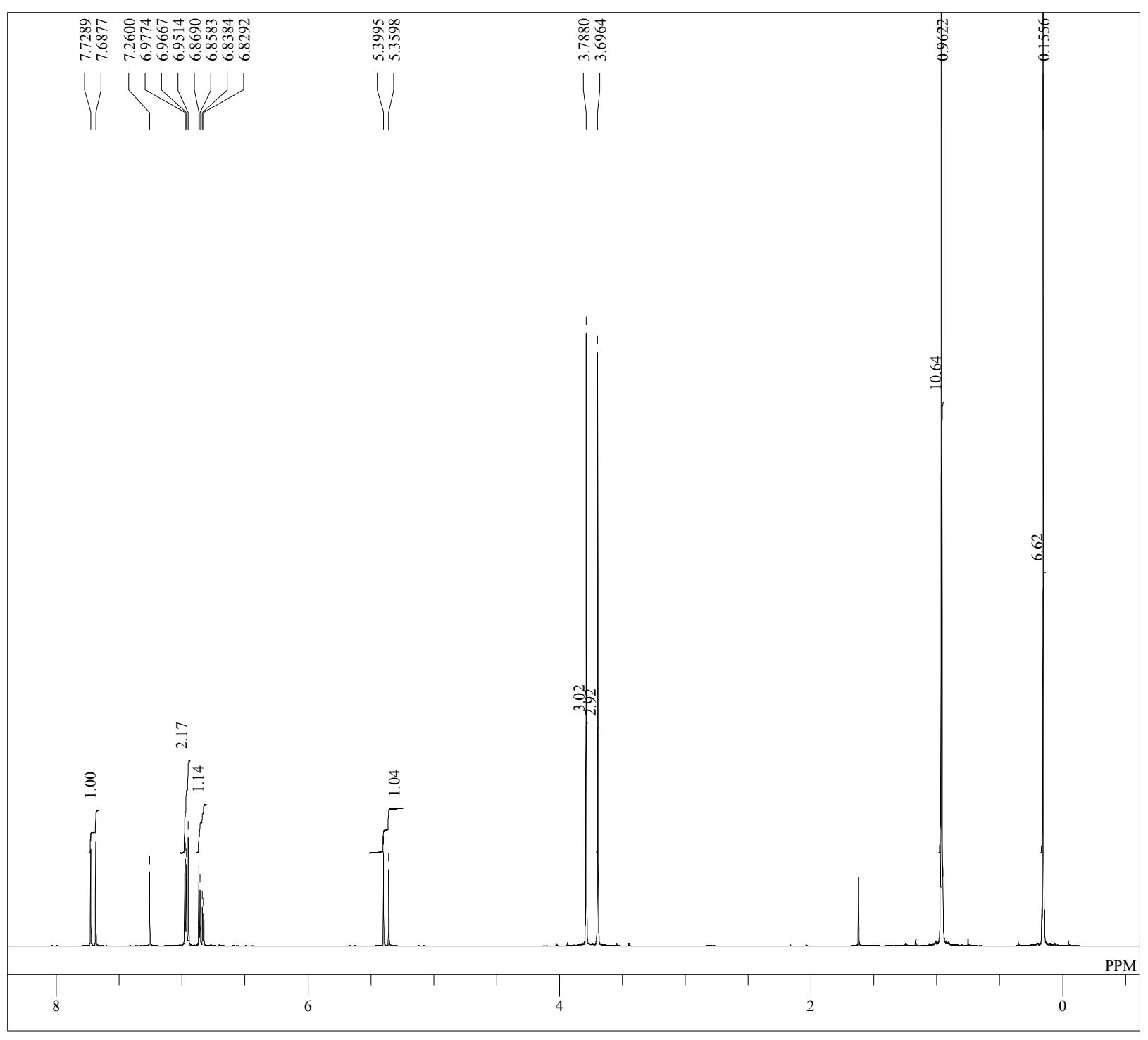

DFILE sub TBS 4-OMe proton-1-1.als

$\begin{array}{ll}\text { DATIM } & 2019-05-13 \text { 22:06:50 } \\ \text { OBNUC } & 1 \mathrm{H}\end{array}$

$\begin{array}{ll}\text { EXMOD } & \text { proton.jxp } \\ \text { OBFRQ } & 300.53 \mathrm{MHz}\end{array}$

$\begin{array}{ll}\text { OBSET } & 1.15 \mathrm{KHz} \\ \text { OBFIN } & 8.57 \mathrm{~Hz}\end{array}$

\begin{tabular}{ll} 
POINT 13107 \\
\hline SCANS
\end{tabular}

FREQU $\quad 6016.85 \mathrm{H} z$

$\begin{array}{ll}\text { SCANS } & 16 \\ \text { ACOTM } & 2.1784 \text { sec }\end{array}$

$\begin{array}{ll}\text { ACQTM } & 2.1784 \mathrm{sec} \\ \mathrm{PD} & 1.0000 \mathrm{sec}\end{array}$

PW1 $\quad 5.50$ usec

$\begin{array}{lll}\text { IRNUC } & 1 \mathrm{H} & \\ \text { CTEMP } & & 19.1 \mathrm{c}\end{array}$

$\begin{array}{ll}\text { CTEMP } & 19.1 \mathrm{c} \\ \text { SLVNT } & \text { CDCL3 }\end{array}$ $\begin{array}{ll}\text { EXREF } & 7.26 \mathrm{ppm} \\ \text { BF } & 0.12 \mathrm{~Hz} \\ \text { RGAIN } & 34\end{array}$

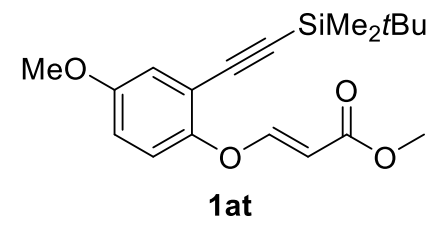

$\mathrm{S} 2-70$ 
single pulse decoupled gated NOE

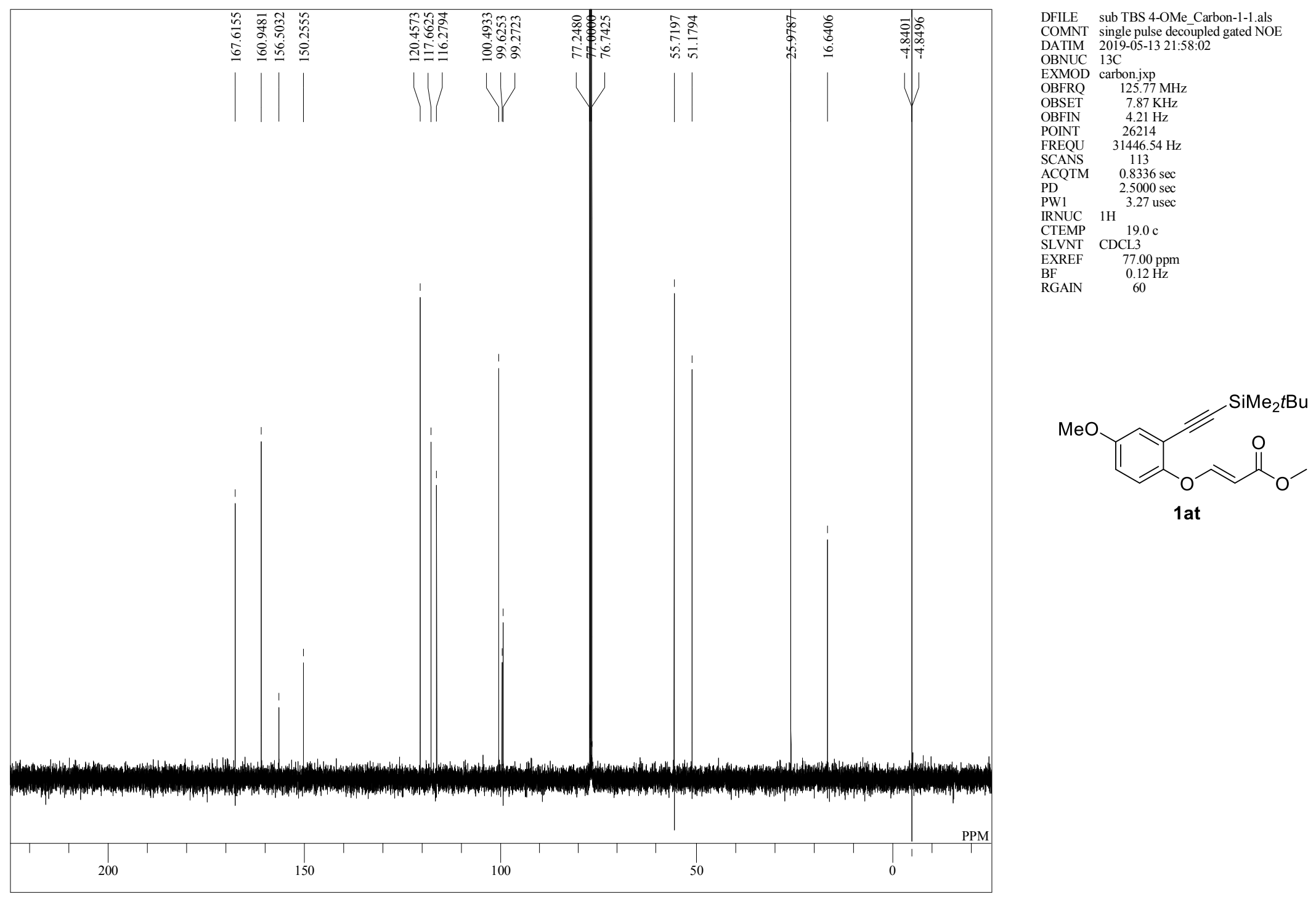


single pulse

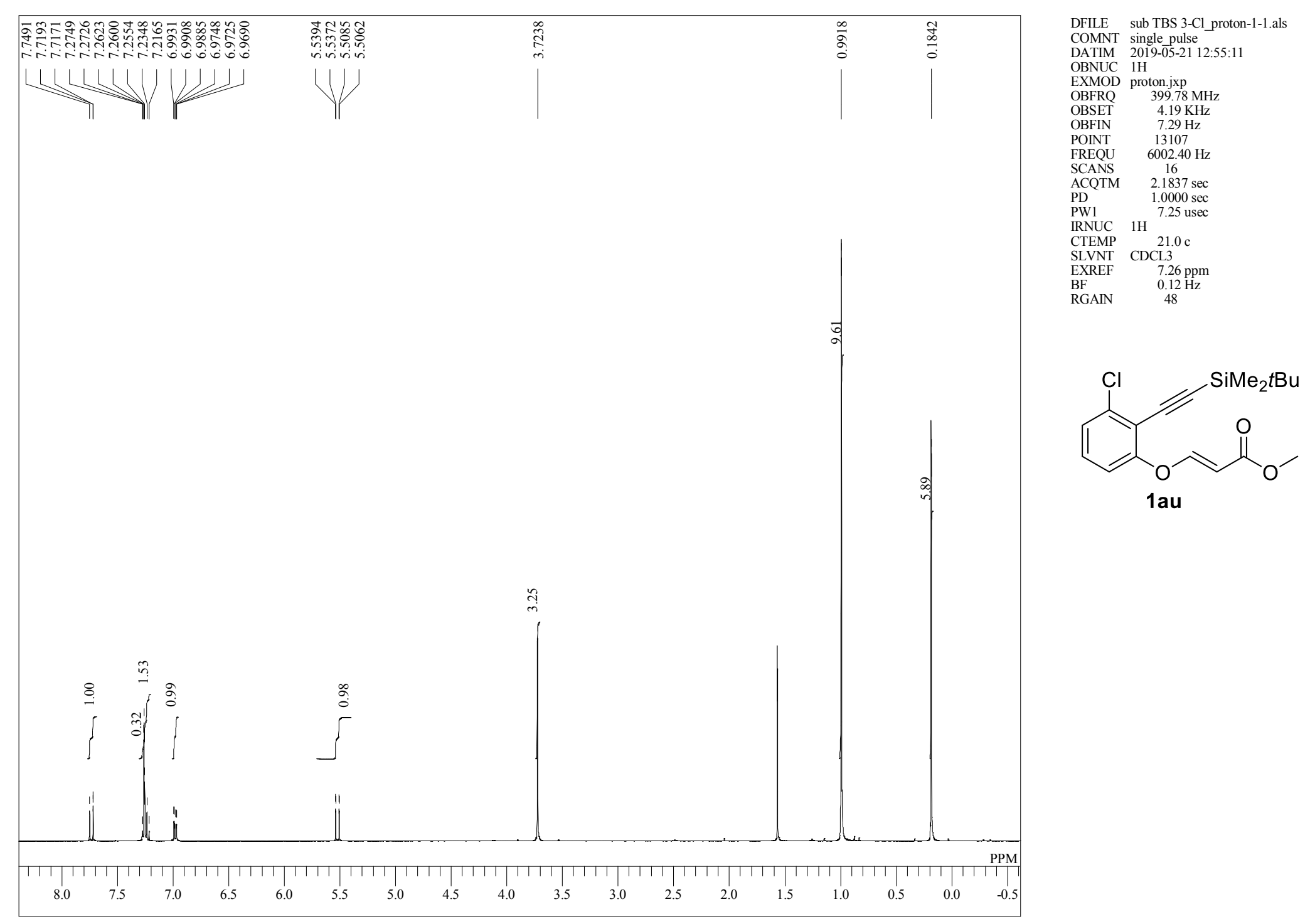


single pulse decoupled gated NOE

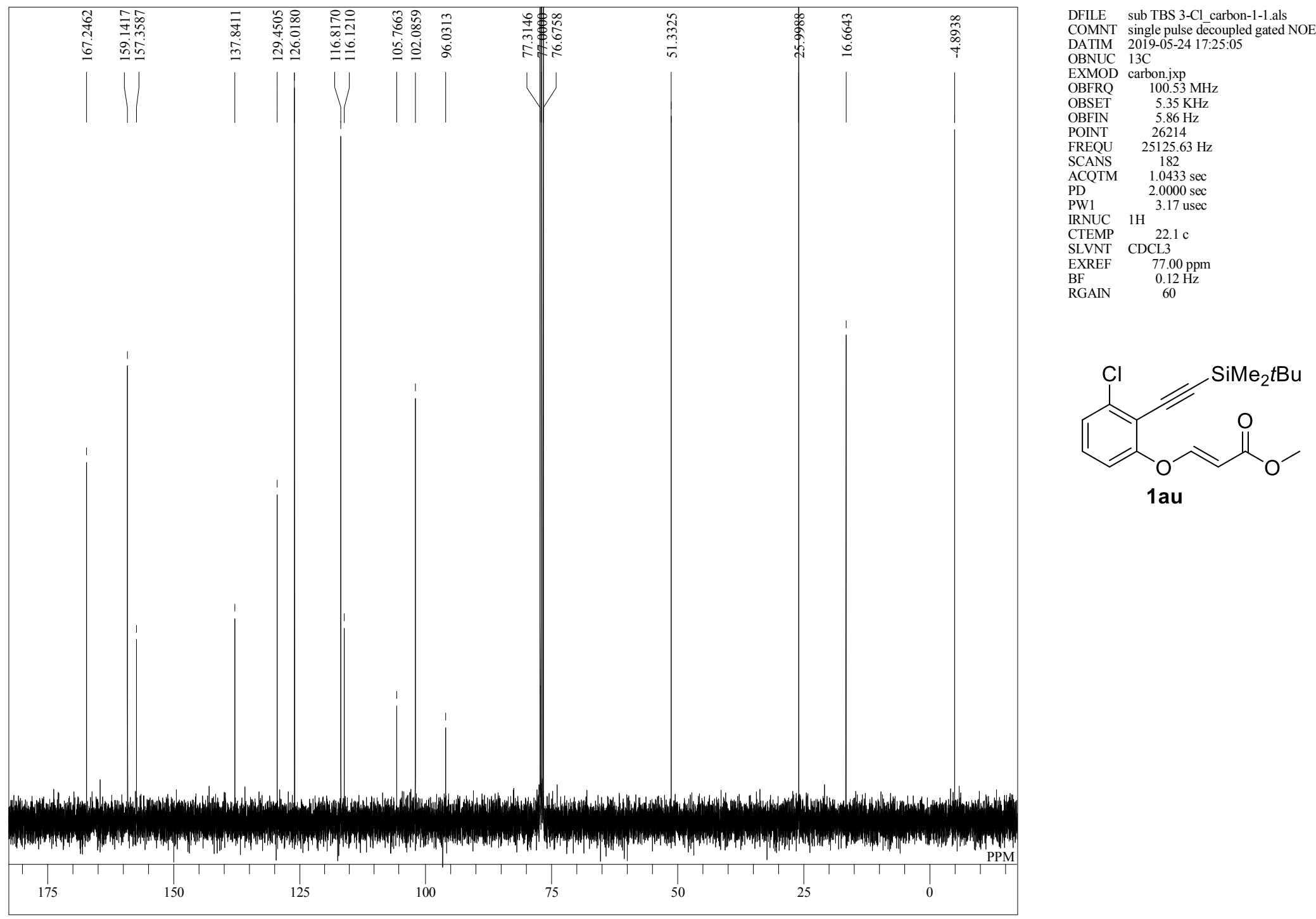


single pulse

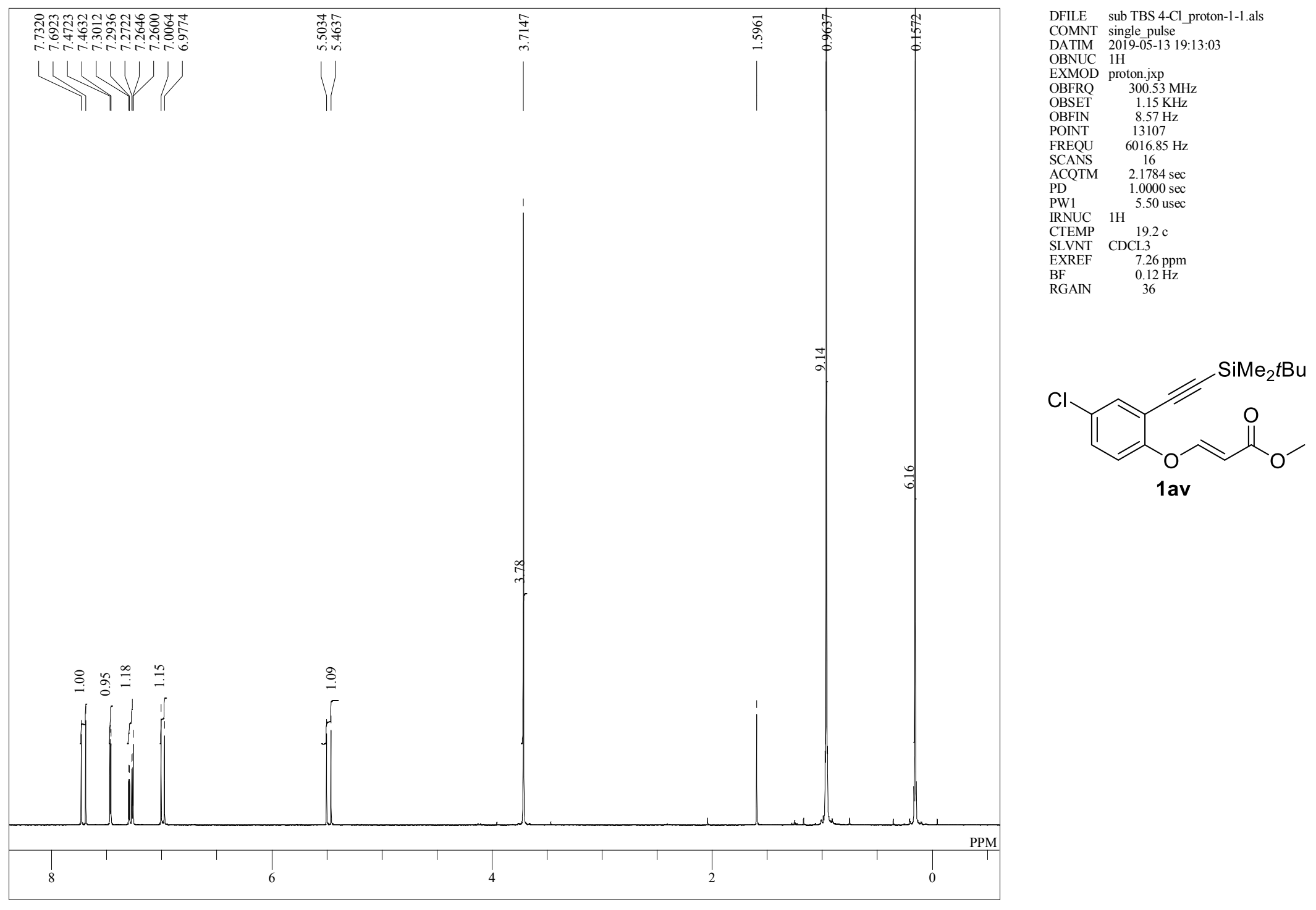

S2 - 74 
single pulse decoupled gated NOE

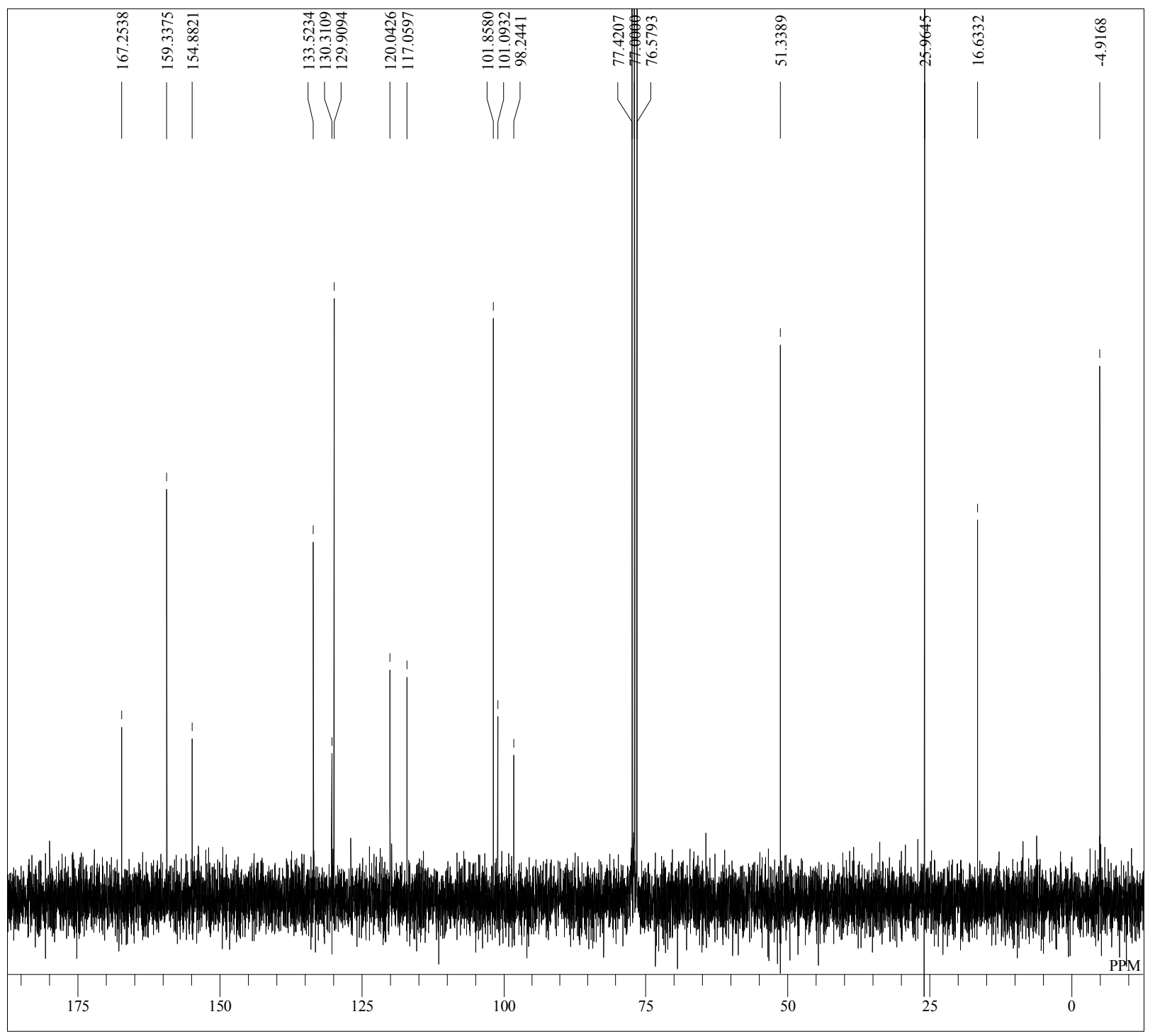

$\begin{array}{ll}\text { DFILE } & \text { sub TBS 4-Cl Carbon-1-1.als } \\ \text { COMNT } & \text { single pulse decoupled gated NOE }\end{array}$

COMNT single pulse decoupled gated $\mathrm{N}$

$\begin{array}{lll}\text { EXMOD } & \text { carbon.jxp } \\ \text { OBFRQ } & 75.57 \mathrm{MHz}\end{array}$

$\begin{array}{ll}\text { OBSET } & 5.79 \mathrm{KH} z \\ \text { OBFIN } & 1.08 \mathrm{~Hz}\end{array}$

\begin{tabular}{ll} 
POINT & $1.08 \mathrm{~Hz}$ \\
\hline & 13107
\end{tabular}

FREQU $\quad 18939.39 \mathrm{~Hz}$

$\begin{array}{ll}\text { SCANS } & 232 \\ \text { ACQTM } & 0.6921 \mathrm{sec}\end{array}$

$\begin{array}{ll}\mathrm{PD} & 0.6921 \mathrm{sec} \\ & 20000 \mathrm{sec}\end{array}$

PW1 $\quad 3.73$ usec

$\begin{array}{lll}\text { IRNUC } & 1 \mathrm{H} & \\ \text { CTEMP } & 19.5 \mathrm{c}\end{array}$

EXREF $77.00 \mathrm{ppm}$

$\begin{array}{lc}\text { BF } & 0.12 \mathrm{~Hz} \\ \text { RGAIN } & 60\end{array}$

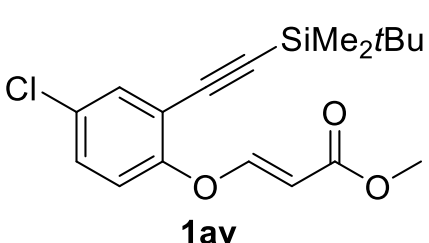


single pulse

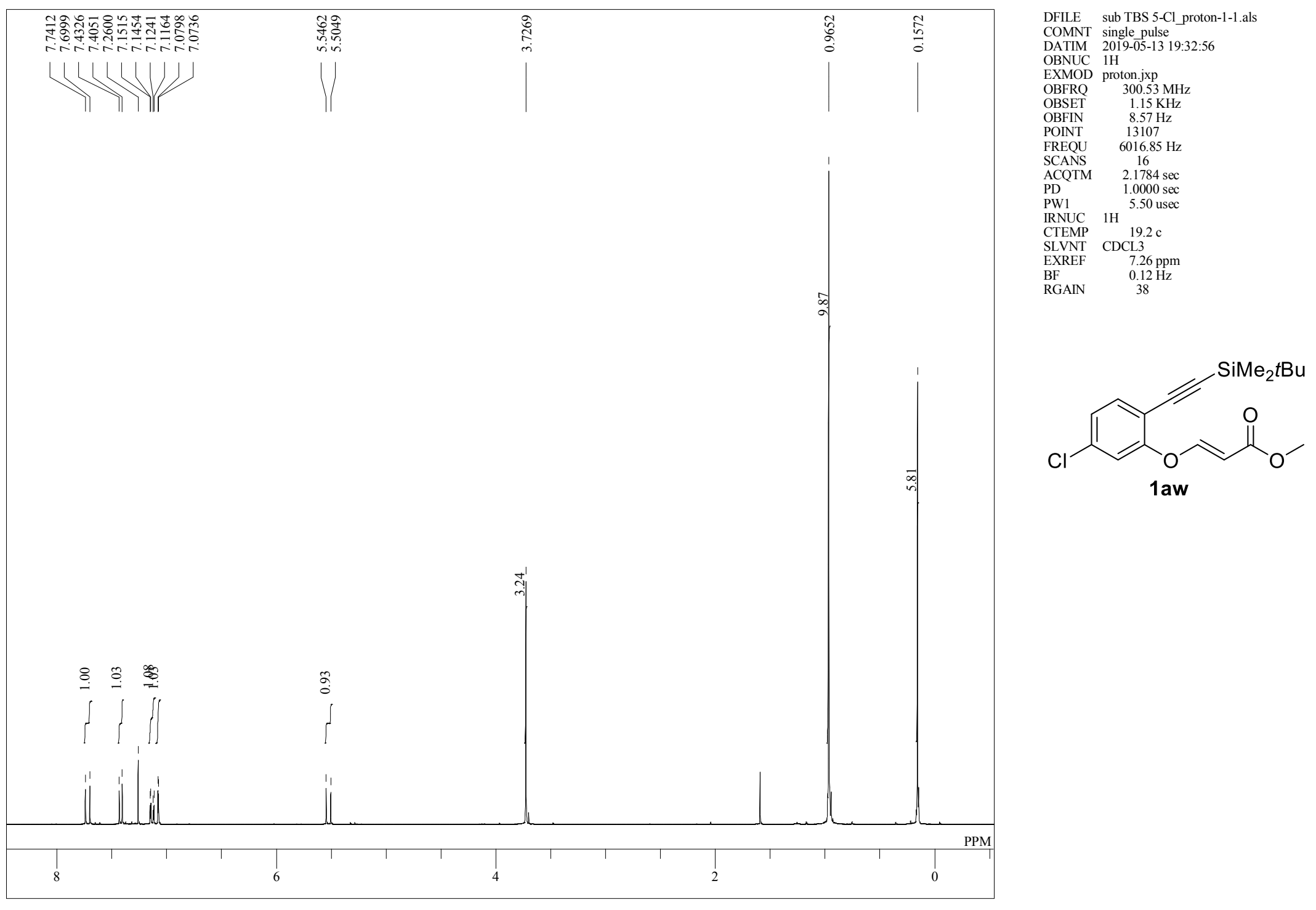

$\mathrm{S} 2-76$ 
single pulse decoupled gated NOE

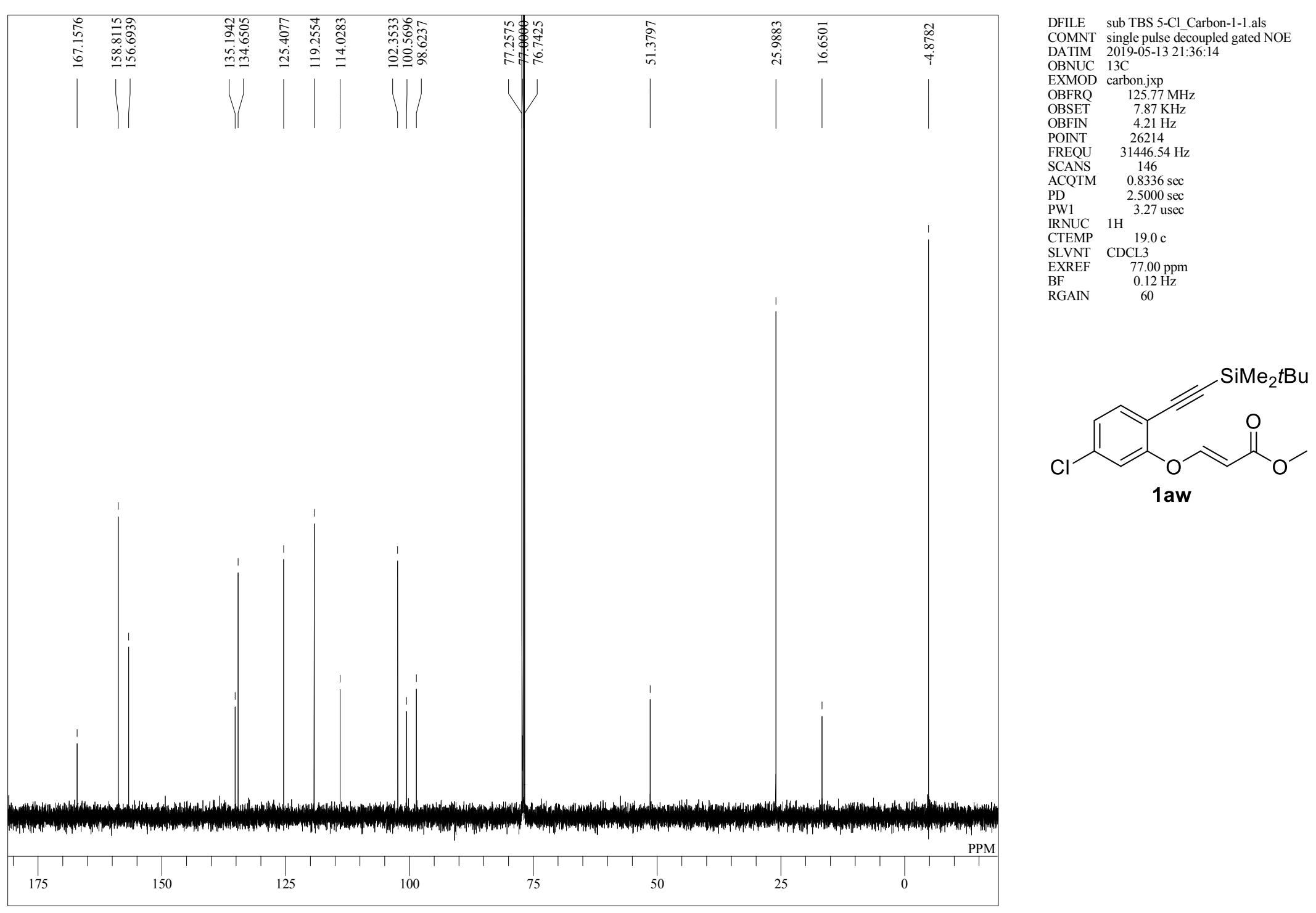

S2 - 77 
single_pulse

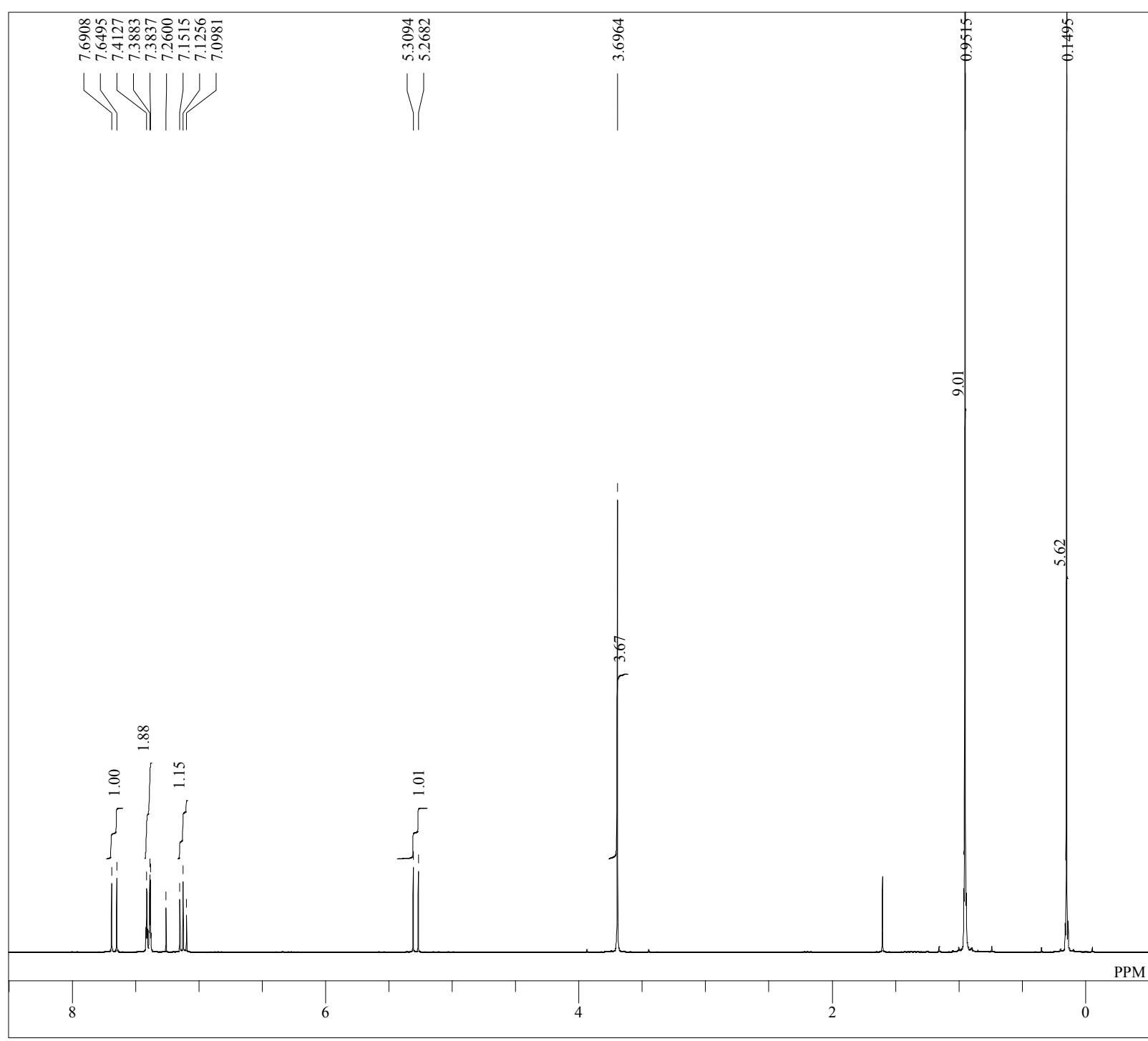

DFILE sub TBS 6-Cl_proton-1-1.als

$\begin{array}{ll}\text { COMNT } & \text { single pulse } \\ \text { DATIM } & 2019-05-13 \text { 19:52:17 }\end{array}$

OBNUC 11

OBFRQ $\quad 300.53 \mathrm{MH} z$

OBSET $\quad 1.15 \mathrm{KHz}$

$\begin{array}{ll}\text { OBFIN } & 8.57 \mathrm{~Hz} \\ \text { POINT } & 13107\end{array}$

FREQU $\quad 6016.85 \mathrm{~Hz}$

$\begin{array}{ll}\text { SCANS } & 16 \\ & \end{array}$

$\begin{array}{ll}\text { ACQTM } & 2.1784 \mathrm{sec} \\ \mathrm{PD} & 10000 \mathrm{sec}\end{array}$

PW1 $\quad 5.50$ usec

IRNUC 1H 5.50 usec

CTEMP $19.2 \mathrm{c}$

$\begin{array}{ll}\text { SLVREF } & 7.26 \mathrm{ppm} \\ \text { EXRE } & 0.12 \mathrm{~Hz}\end{array}$

$\begin{array}{lc}\text { EXREF } & 7.26 \mathrm{pp} \\ \text { BF } & 0.12 \mathrm{~Hz} \\ \text { RGAIN } & 36\end{array}$

(n)

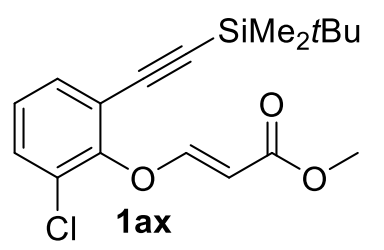

S2 - 78 
single pulse decoupled gated NOE

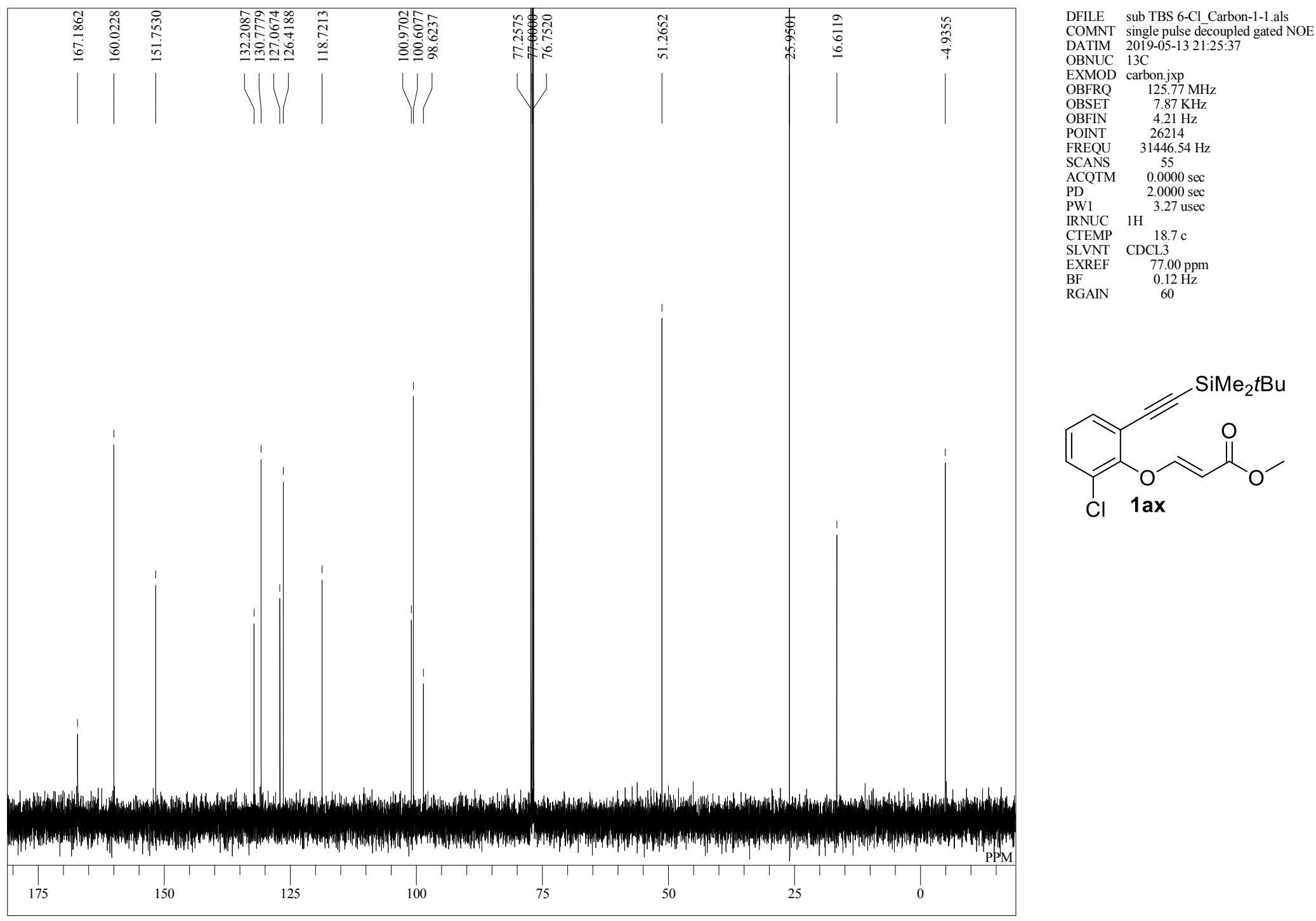


single pulse

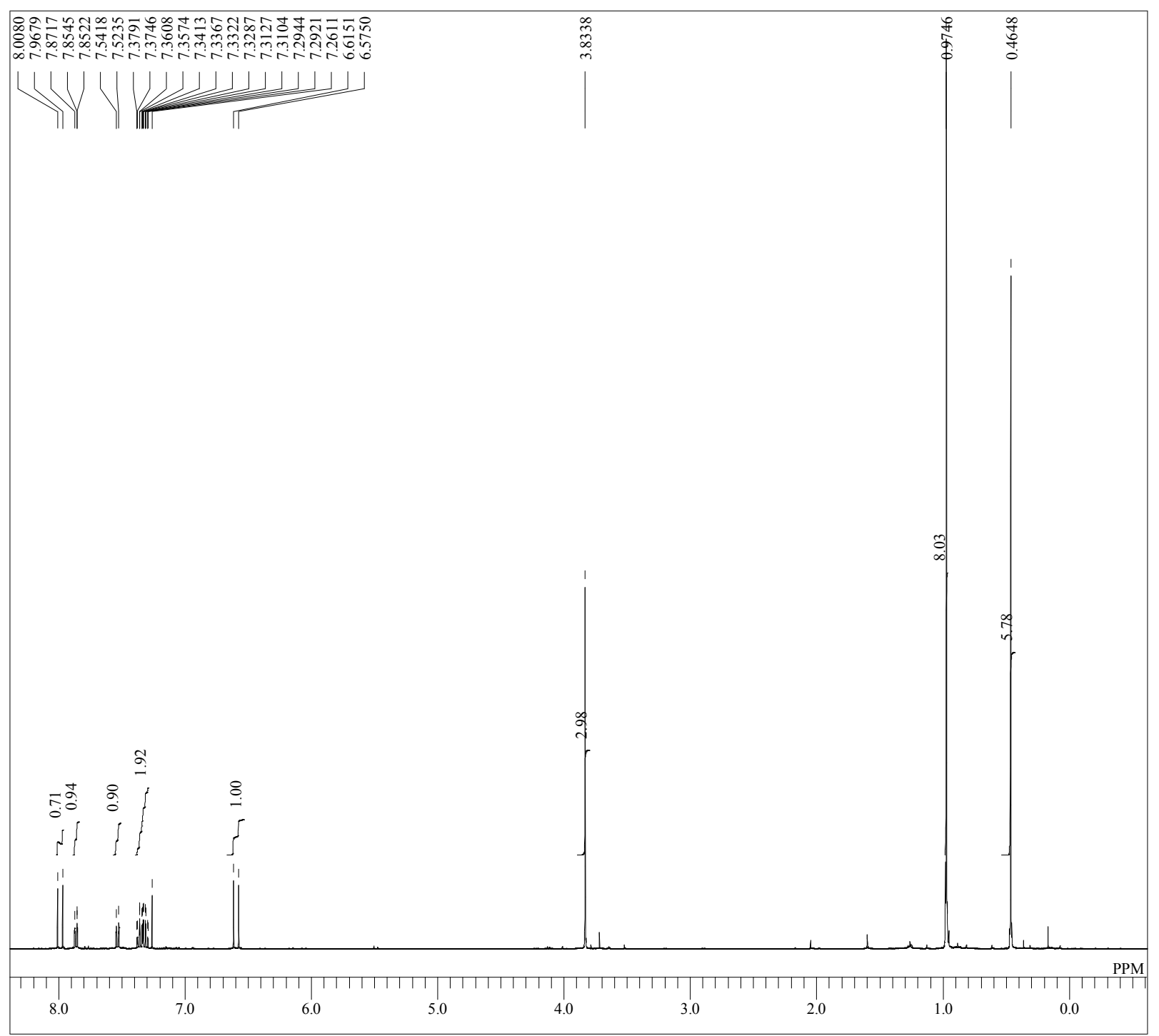

S2 - 80
DFILE tbsprod proton-1-1.als

$\begin{array}{ll}\text { DATIM } & \text { single-pulse } \\ \text { 2018-07-24 14:50:10 }\end{array}$

OBFRQ proton.jxp $399.78 \mathrm{MHz}$

OBSET $\quad 4.19 \mathrm{KHz}$

$\begin{array}{ll}\text { OBFIN } & 7.29 \mathrm{~Hz} \\ \text { POINT } & 13107\end{array}$

FREQU $\quad 6002.40 \mathrm{~Hz}$

$\begin{array}{ll}\text { SCANS } & 16 \\ \text { ACQTM } & 2.1837 \mathrm{sec}\end{array}$

$\begin{array}{ll}\text { ACQTM } & 2.1837 \mathrm{sec} \\ \mathrm{PD} & 1.0000 \mathrm{sec}\end{array}$

$\begin{array}{ll}\text { PD } & 1.0000 \mathrm{sec} \\ \text { PW1 } & 7.25 \mathrm{usec}\end{array}$

IRNUC $1 \mathrm{H} .25$ usc

CTEMP $19.7 \mathrm{c}$

$\begin{array}{ll}\text { EXREF } & 7.26 \mathrm{ppm} \\ \mathrm{BF} & 0.12 \mathrm{H} Z\end{array}$

RGAIN $\quad 0.12 \mathrm{~Hz}$

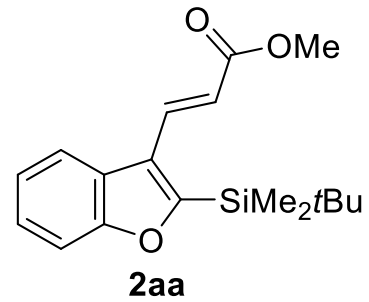


single pulse decoupled gated NOE

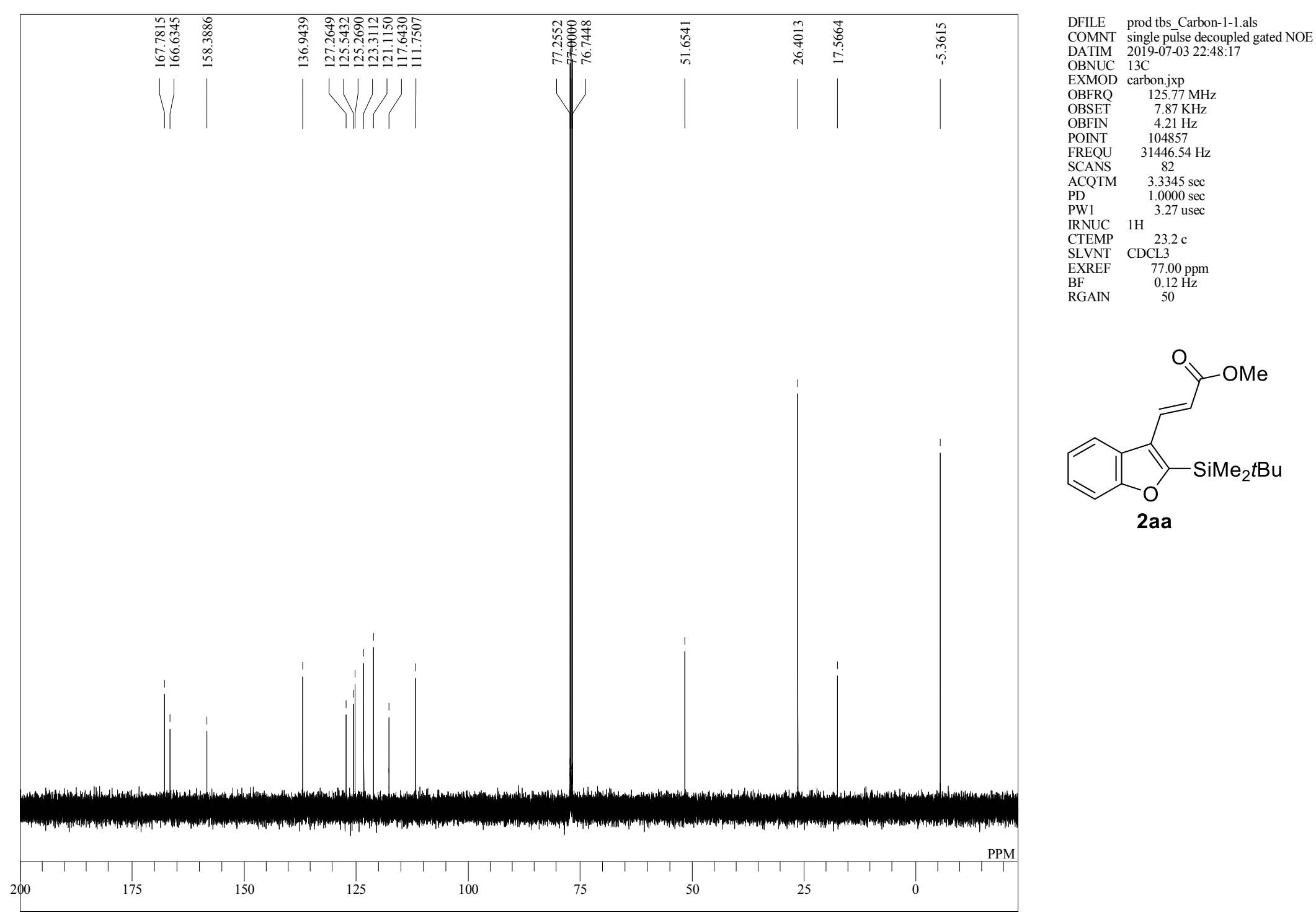


single pulse

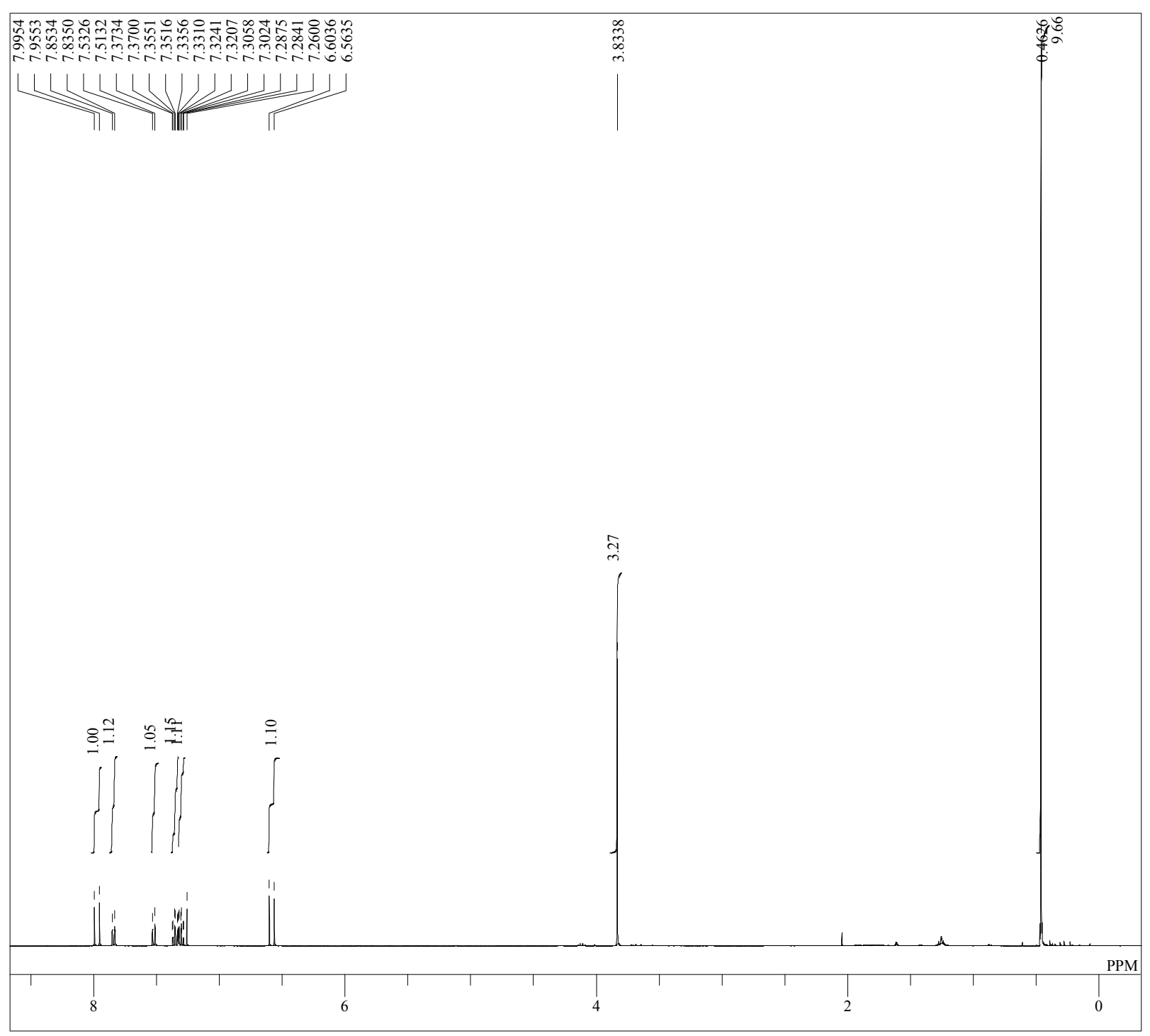

DFILE prod TMS proton-1-1.als

COMNT single pulse $23 \cdot 08 \cdot 46$

OBNUC $1 \mathrm{H}$

EXMRD proton.jxp

OBSET $\quad 4.19 \mathrm{KHz}$

POINT $\quad 13107$

FREQU $\quad 6002.40 \mathrm{~Hz}$

SCANS 16

$2.1837 \mathrm{sec}$
$\mathrm{PD}$

$\begin{array}{ll}\text { PD } & 5.0000 \mathrm{sec} \\ \text { PW1 } & 5.64 \text { usec }\end{array}$

IRNUC 1H

CTEMP $19.6 \mathrm{c}$

EXREF $7.26 \mathrm{ppm}$

$\begin{array}{ll}\text { BF } & 0.12 \mathrm{~Hz} \\ \text { RGAIN } & 38\end{array}$

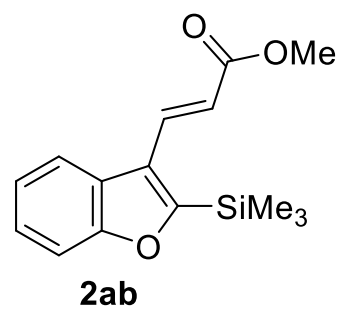

$2 \mathrm{ab}$ 
single pulse decoupled gated NOE

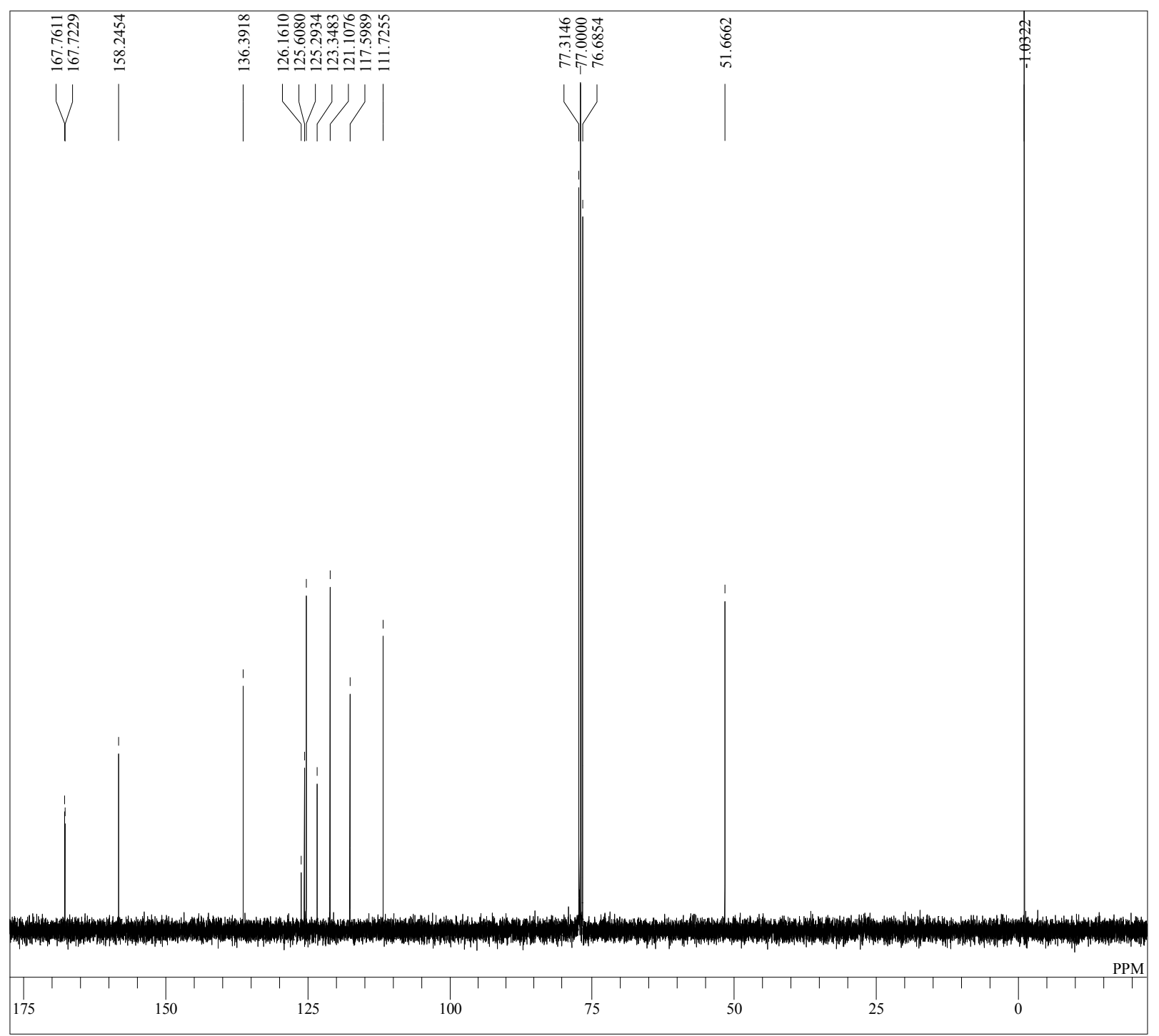

DFILE prod TMS carbon-1-1.als

COMTIT single pulse decoupled gated NOE

OBNUC 13

EXMOD carbon.jxp

$\begin{array}{ll}100.53 \mathrm{MHz} \\ \text { OBSET } & 5.35 \mathrm{KHz}\end{array}$

$\begin{array}{ll}\text { OBFIN } & 5.86 \mathrm{H} z \\ \text { PONT } & 26214\end{array}$

FREQU $\quad 25125.63 \mathrm{~Hz}$

$\begin{array}{ll}\text { SCANS } & 227 \\ \text { ACQTM } & 1.0433 \mathrm{sec}\end{array}$

$\begin{array}{ll}\text { ACQTM } & 1.0433 \mathrm{sec} \\ \mathrm{PD} & 2.0000 \mathrm{sec}\end{array}$

$\begin{array}{ll}\text { PW1 } & 2.0000 \mathrm{sec} \\ \text { PW1 } & 3.05 \mathrm{usec}\end{array}$

$\begin{array}{lll}\text { IRNUC } & 1 \mathrm{H} & \\ \text { CTEMP } & 20.0 \mathrm{c}\end{array}$

SLVNT CDCL3 3

EXREF $\quad 77.00 \mathrm{ppm}$

$\begin{array}{lc}\text { BF } & 0.12 \mathrm{~Hz} \\ \text { RGAIN } & 60\end{array}$

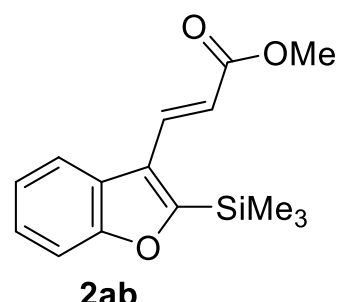

$2 a b$ 
single pulse

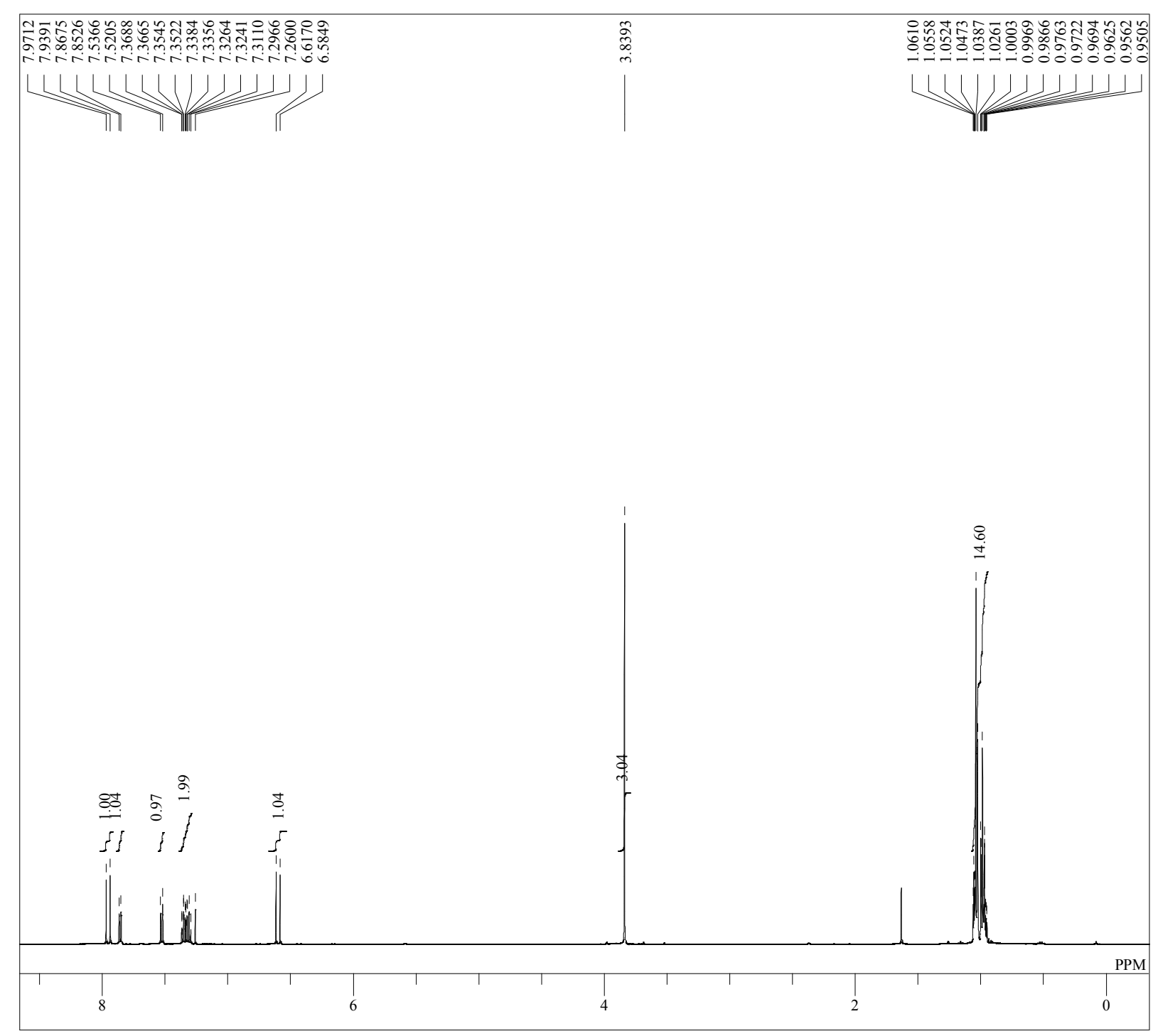

DFILE tes prod_proton-1-1.als

COMNT single

OBNUC $1 \mathrm{H}$

OBNUC $1 \mathrm{H}$

OBFRQ $500.16 \mathrm{MHz}$

OBSET $\quad 2.41 \mathrm{KHz}$

$\begin{array}{ll}\text { OBFIN } & 6.01 \mathrm{~Hz} \\ \text { POINT } & 26214\end{array}$

FREQU $\quad 7507.51 \mathrm{H} z$

SCANS 16

$3.4918 \mathrm{sec}$
PCQTM

$\begin{array}{ll}2.0000 \mathrm{sec} \\ \text { PW1 } & 6.50 \mathrm{usec}\end{array}$

IRNUC 1H

CTEMP 23.0

$\begin{array}{ll}\text { EXREF } & \text { CDCL3 } \\ & \text { C.26 ppm }\end{array}$

BF

$7.26 \mathrm{ppm}$
$0.12 \mathrm{~Hz}$
36

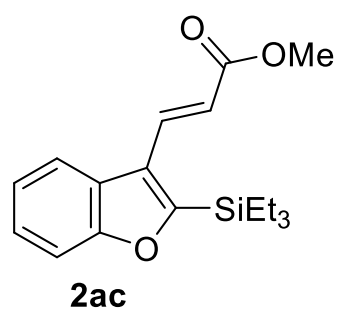


single pulse decoupled gated NOE

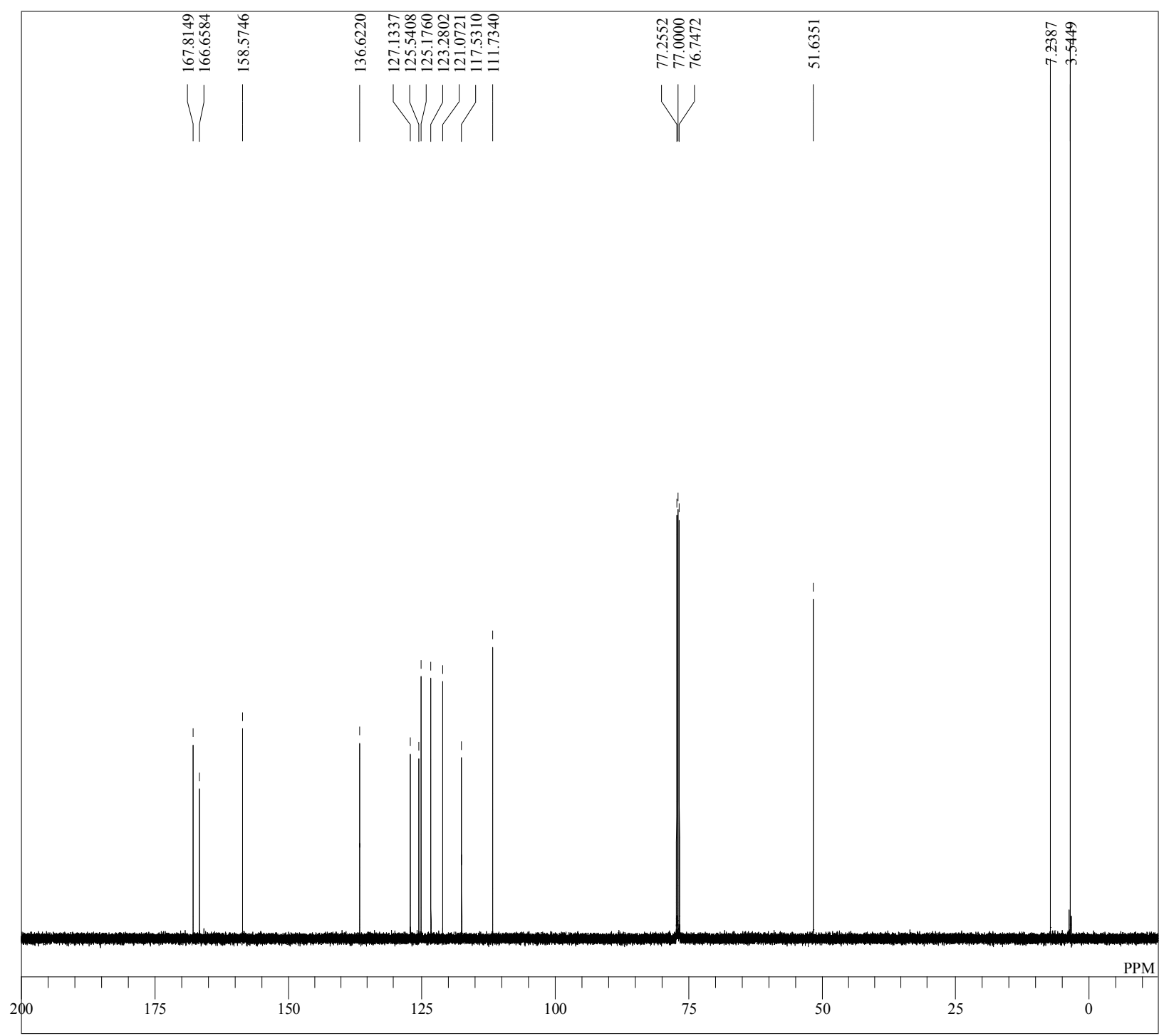

DFILE tes prod_Carbon-1-1.als

COMNT single pulse decoupled gated NOE

EXMOD

OBFRQ $\quad 125.77 \mathrm{MHz}$

OBSET $\quad 7.87 \mathrm{KHz}$

POINT 104857

FREQU $\quad 31446.54 \mathrm{~Hz}$

SCANS $\quad 200$

$\begin{array}{ll}\text { ACQTM } & 3.3345 \mathrm{sec} \\ \mathrm{PD} & 1.0000 \mathrm{sec}\end{array}$

$\begin{array}{ll}\text { PD } & 1.0000 \mathrm{sec} \\ \text { PW1 } & 3.27 \text { usec }\end{array}$

$\begin{array}{lll}\text { IRNUC } & 1 \mathrm{H} & 3.27 \\ \text { CTEMP } & & 23.3 \mathrm{c}\end{array}$

$\begin{array}{ll}\text { SLVNT } & \text { CDCL3 } \\ \text { EXREF } & 77.00 \mathrm{ppm}\end{array}$

$\begin{array}{lc}\text { EXREF } & 77.00 \mathrm{ppm} \\ \text { BF } & 0.12 \mathrm{~Hz} \\ \text { RGAIN } & 56\end{array}$

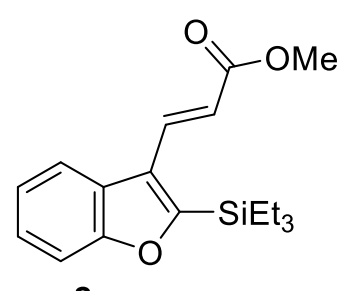

2ac 
single pulse

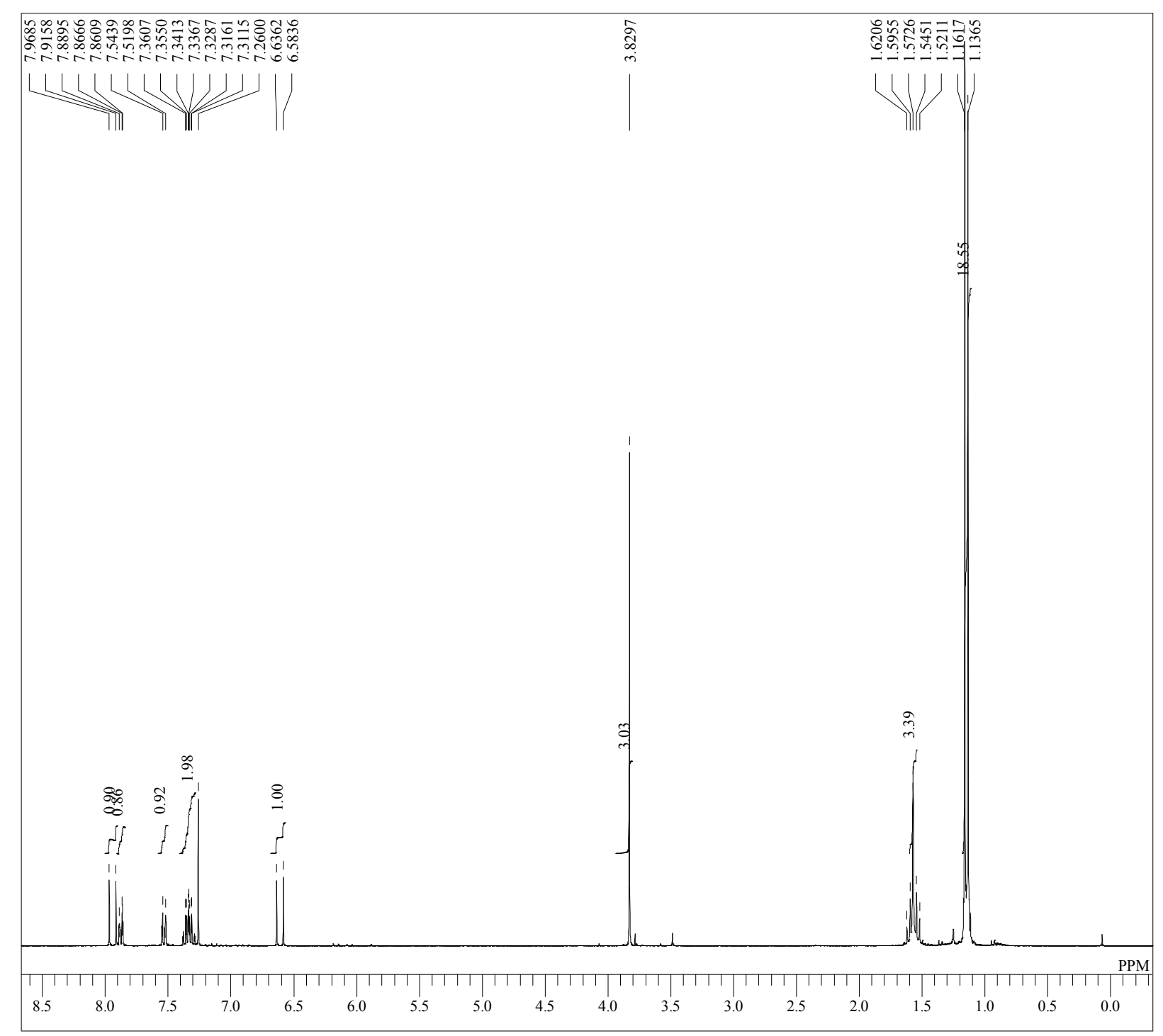

DFILE prod tips proton-1-1.als

COMNT single pulse 1 -1-1.58

OBNUC $1 \mathrm{H}$

EXMRO proton.jxp

OBSET $\quad 1.15 \mathrm{KHz}$

$\begin{array}{ll}\text { POINT } & 13107 \\ \text { FREQU } & 4508.57 \mathrm{~Hz}\end{array}$

$\begin{array}{lc}\text { FREQU } & 4508.57 \mathrm{~Hz} \\ \text { SCANS } & 16\end{array}$

$\begin{array}{ll}\text { ACQTM } & 2.9072 \mathrm{sec} \\ \mathrm{PD} & 1.0000 \mathrm{sec}\end{array}$

$\begin{array}{ll}\text { PD } & 1.0000 \mathrm{sec} \\ \text { PW1 } & 5.50 \mathrm{usec}\end{array}$

IRNUC $1 \mathrm{H}$

CTEMP $21.4 \mathrm{c}$

EXREF $7.26 \mathrm{ppm}$

$\begin{array}{ll}\text { BF } & 7.26 \mathrm{ppm} \\ \text { RGAIN } & 44\end{array}$

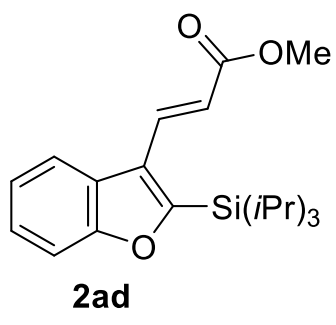

S2 - 86 
single pulse decoupled gated NOE

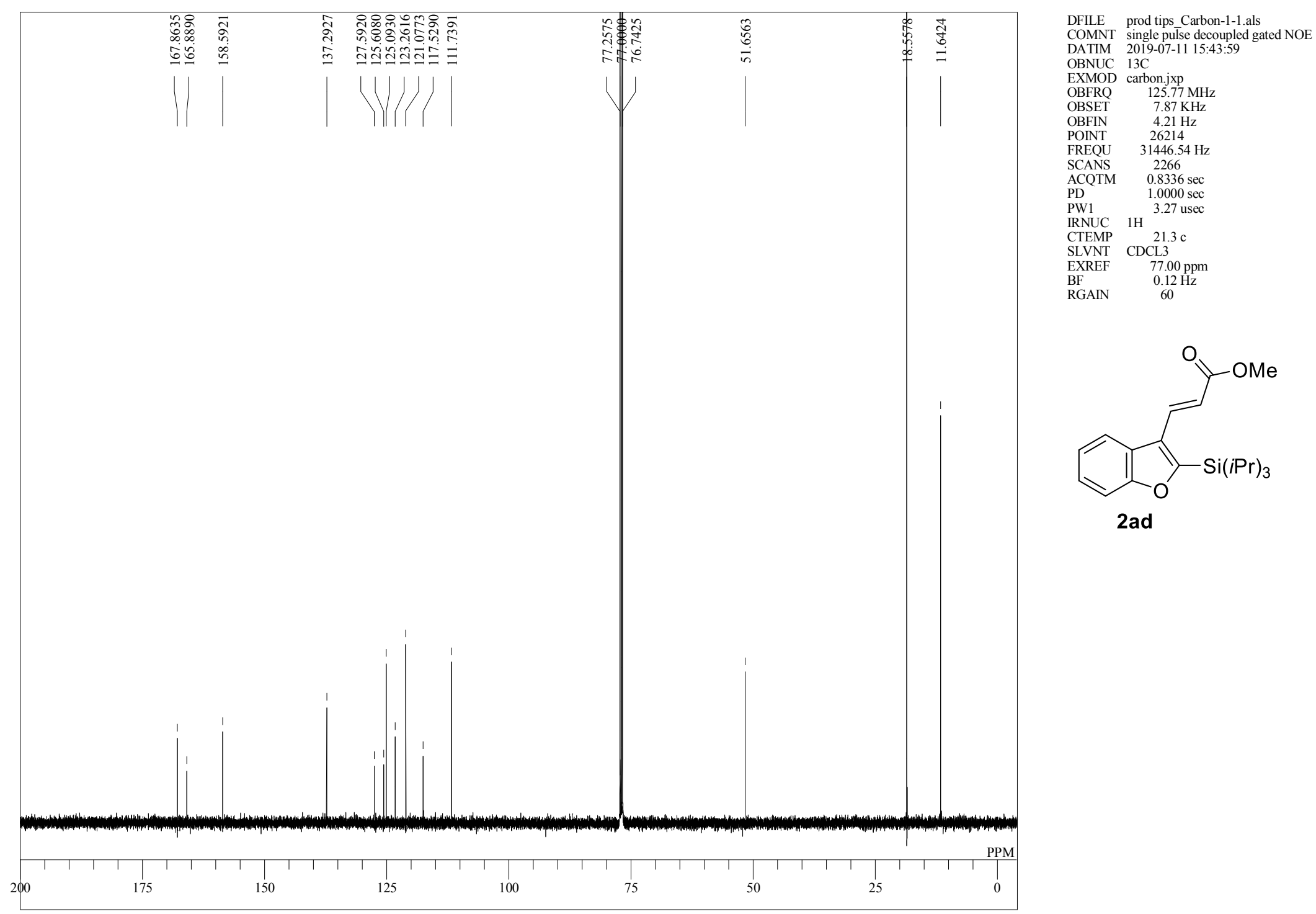


single _pulse

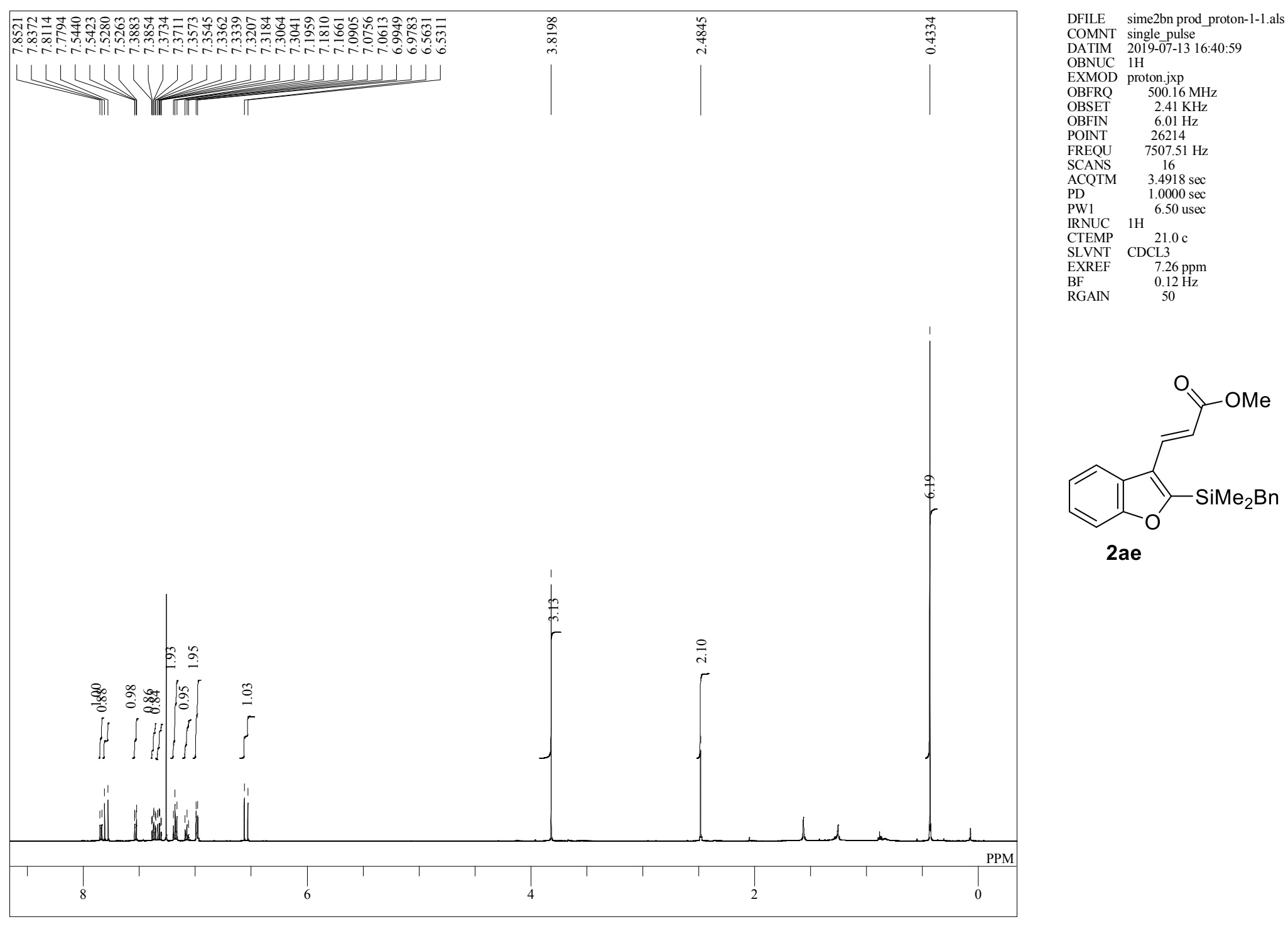


single pulse decoupled gated NOE

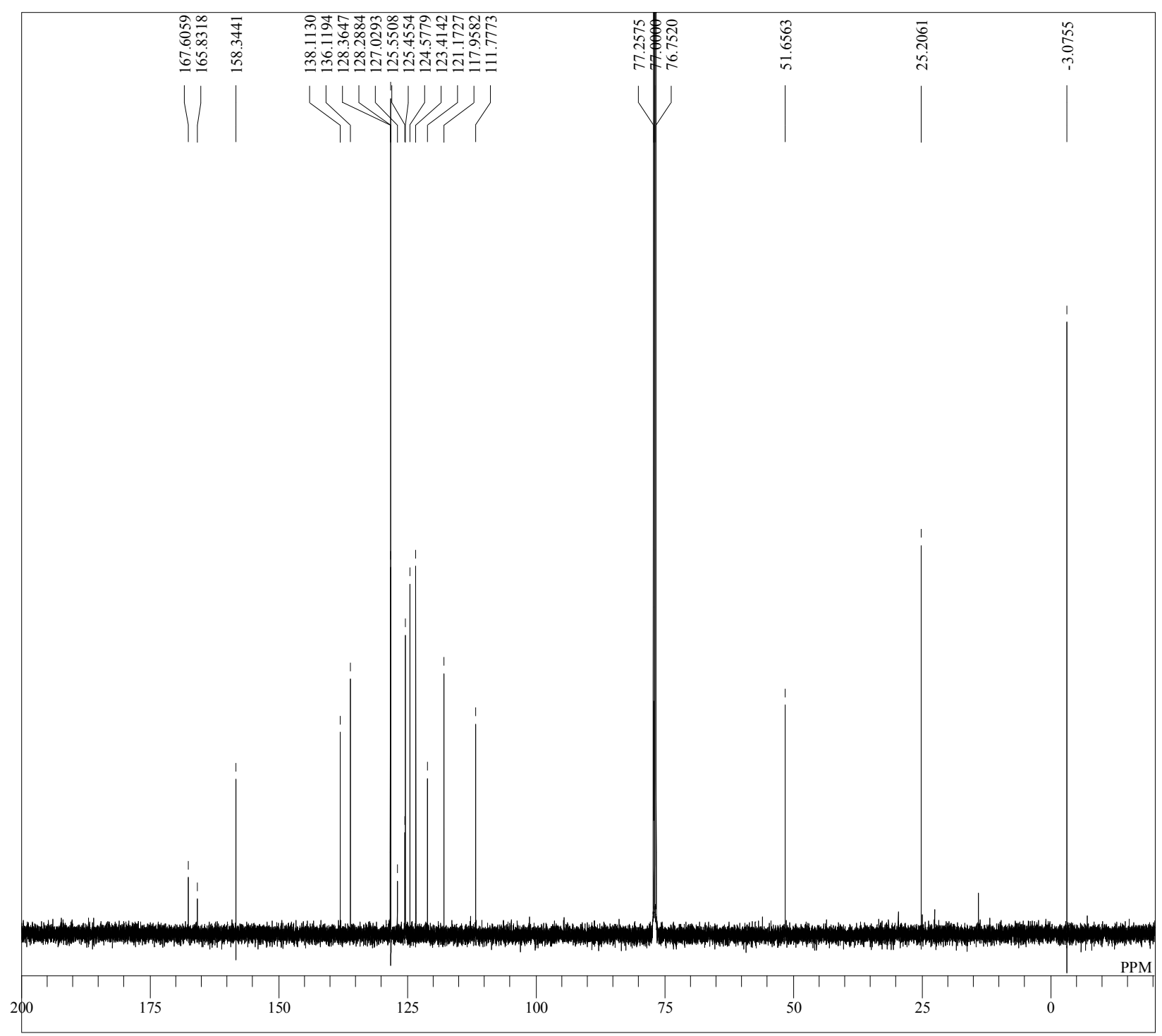

DFILE sime2bn prod_Carbon-1-1.als

OBNUC 13C

OBFRQ $\quad 125.77 \mathrm{MHz}$

OBSET $\quad 7.87 \mathrm{KH} z$

POINT 26214

FREQU $31446.54 \mathrm{~Hz}$

$\begin{array}{ll}\text { SCANS } & 2678 \\ \text { ACQTM } & 0.8336 \mathrm{sec}\end{array}$

$\mathrm{PD} \quad 1.0000 \mathrm{sec}$

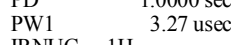

IRNEM $1 \mathrm{H} 21.0$

CTEMP 21.0

EXREF $\quad 77.00 \mathrm{ppm}$

$\begin{array}{lc}\text { BF } & 0.12 \mathrm{~Hz} \\ \text { RGAIN } & 60\end{array}$

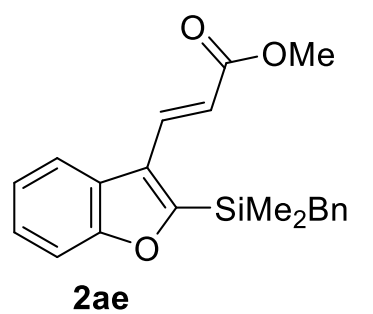

2ae 
single pulse

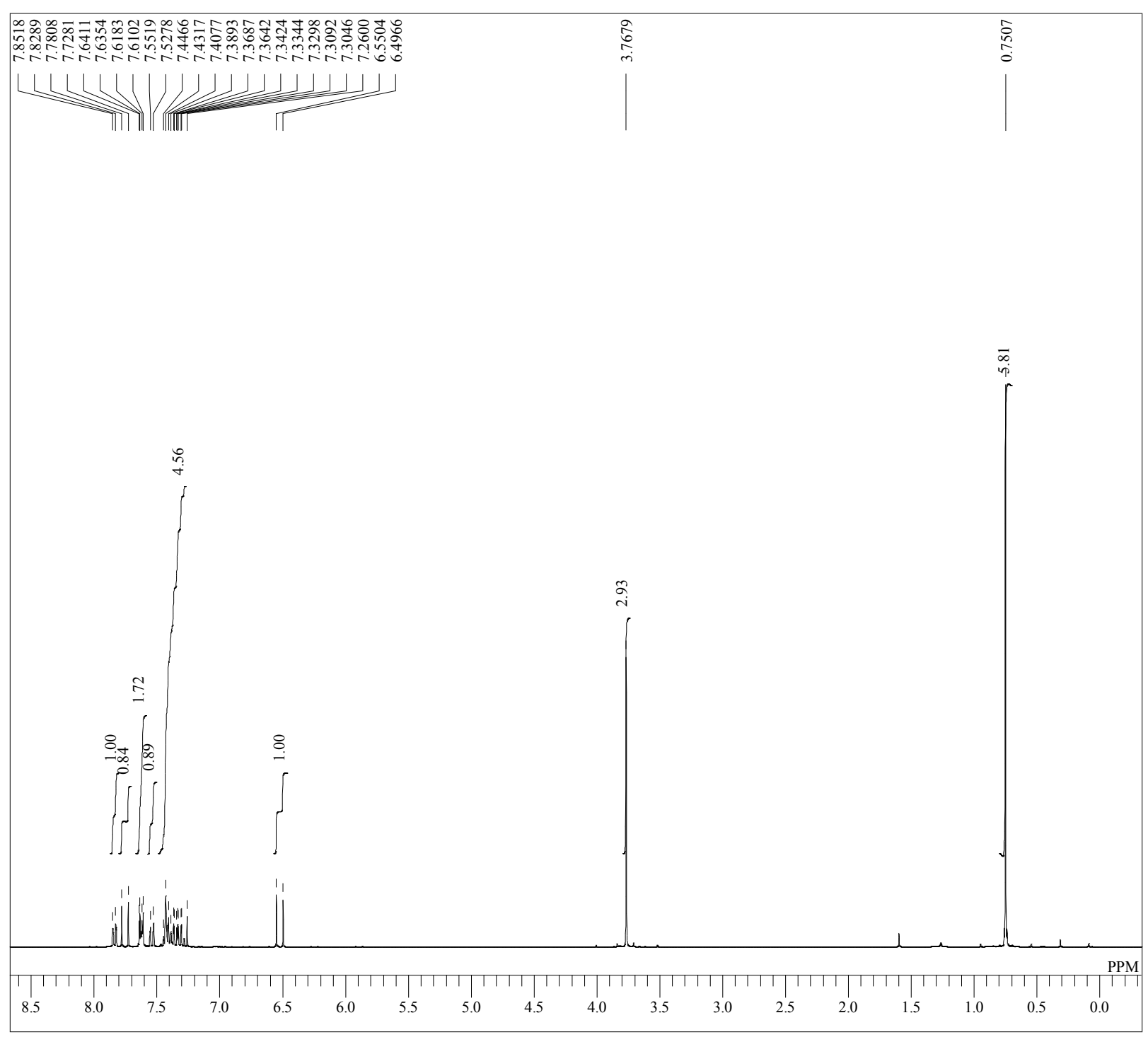

DFILE prod sime2ph_proton-1-1.als

COMNT single pulse

OBNUC $1 \mathrm{H}$

$\begin{array}{ll}\text { EXMOD proton.jxp } \\ \text { OBFRQ } & 300.53 \mathrm{MHz}\end{array}$

$\begin{array}{ll}\text { OBSET } & 1.15 \mathrm{KHz} \\ \text { OBFIN } & 857 \mathrm{~Hz}\end{array}$

$\begin{array}{ll}\text { OBFIN } & 8.57 \mathrm{~Hz} \\ \text { POINT } & 13107\end{array}$

FREQU $\quad 4508.57 \mathrm{~Hz}$

SCANS 16

$2.9072 \mathrm{sec}$
$\mathrm{PD}$

$\begin{array}{ll}\text { PW1 } & 1.0000 \mathrm{sec} \\ \text { PWe } & \text { usec }\end{array}$

IRNUC $1 \mathrm{H} 21.4 \mathrm{c}$

$\begin{array}{ll}\text { CTEMP } & 21.4 \mathrm{C} \\ \text { SLVNT } & \text { CDCL3 }\end{array}$

$\begin{array}{ll}\text { EXREF } & 7.26 \mathrm{ppm} \\ \mathrm{BF} & 0.12 \mathrm{~Hz}\end{array}$

$\begin{array}{ll}\text { BF } & 0.12 \mathrm{~Hz} \\ \text { RGAIN } & 38\end{array}$

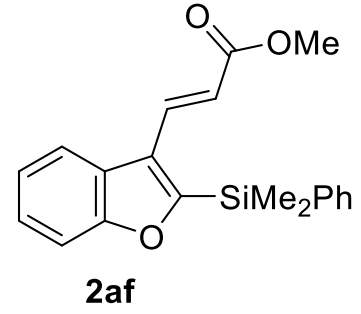

S2 - 90 
single pulse decoupled gated NOE

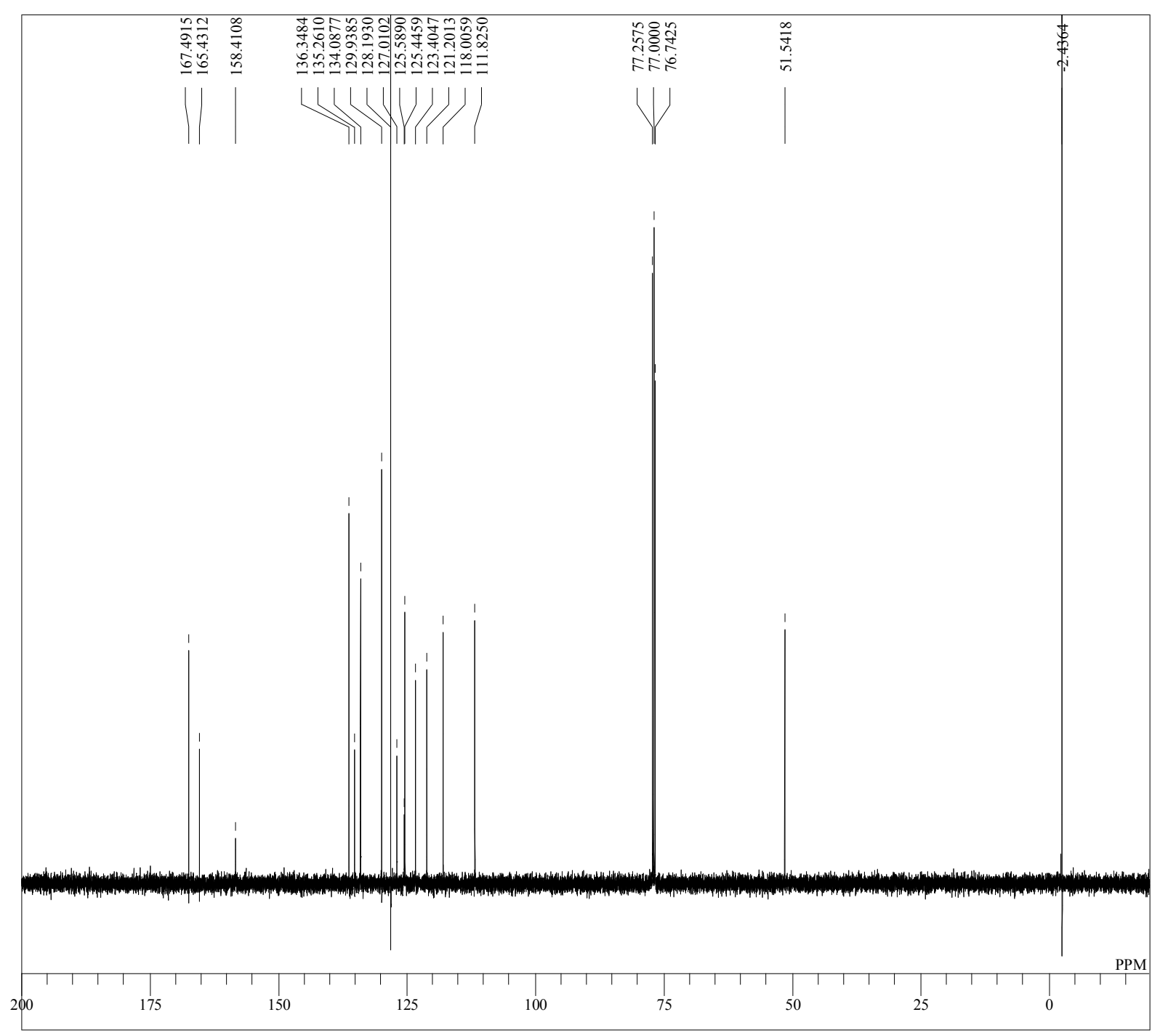

DFILE prod sime2ph Carbon-1-1.als

DATIM 2019-07-11 15:30:03

OBNUC $13 \mathrm{C}$

OBFRO $\quad 125.77 \mathrm{MHz}$

$\begin{array}{ll}\text { OBFRQ } & 125.77 \mathrm{MHz} \\ \text { OBSET } & 7.87 \mathrm{KHz}\end{array}$

$\begin{array}{ll}\text { OBFIN } & 4.21 \mathrm{~Hz} \\ \text { POINT } & 26214\end{array}$

FREQU $\quad 31446.54 \mathrm{~Hz}$

ACQTM $0.8336 \mathrm{sec}$

$\begin{array}{ll}\mathrm{PD} & 1.000 \mathrm{sec} \\ \mathrm{PW} & 3.27 \mathrm{sec}\end{array}$

IRNUC 1H

$\begin{array}{ll}\text { CTEMP } & 20.9 \mathrm{c} \\ \text { SLVNT } & \text { CDCL3 }\end{array}$

$\begin{array}{ll}\text { SLVNT } & \text { CDCL3 } \\ \text { EXREF } & 77.00 \mathrm{ppm}\end{array}$

$\begin{array}{lc}\text { EXREF } & 77.00 \mathrm{ppm} \\ \text { BF } & 0.12 \mathrm{~Hz} \\ \text { RGAIN } & 60\end{array}$

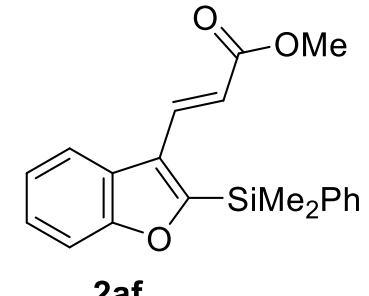

2af 
single _pulse

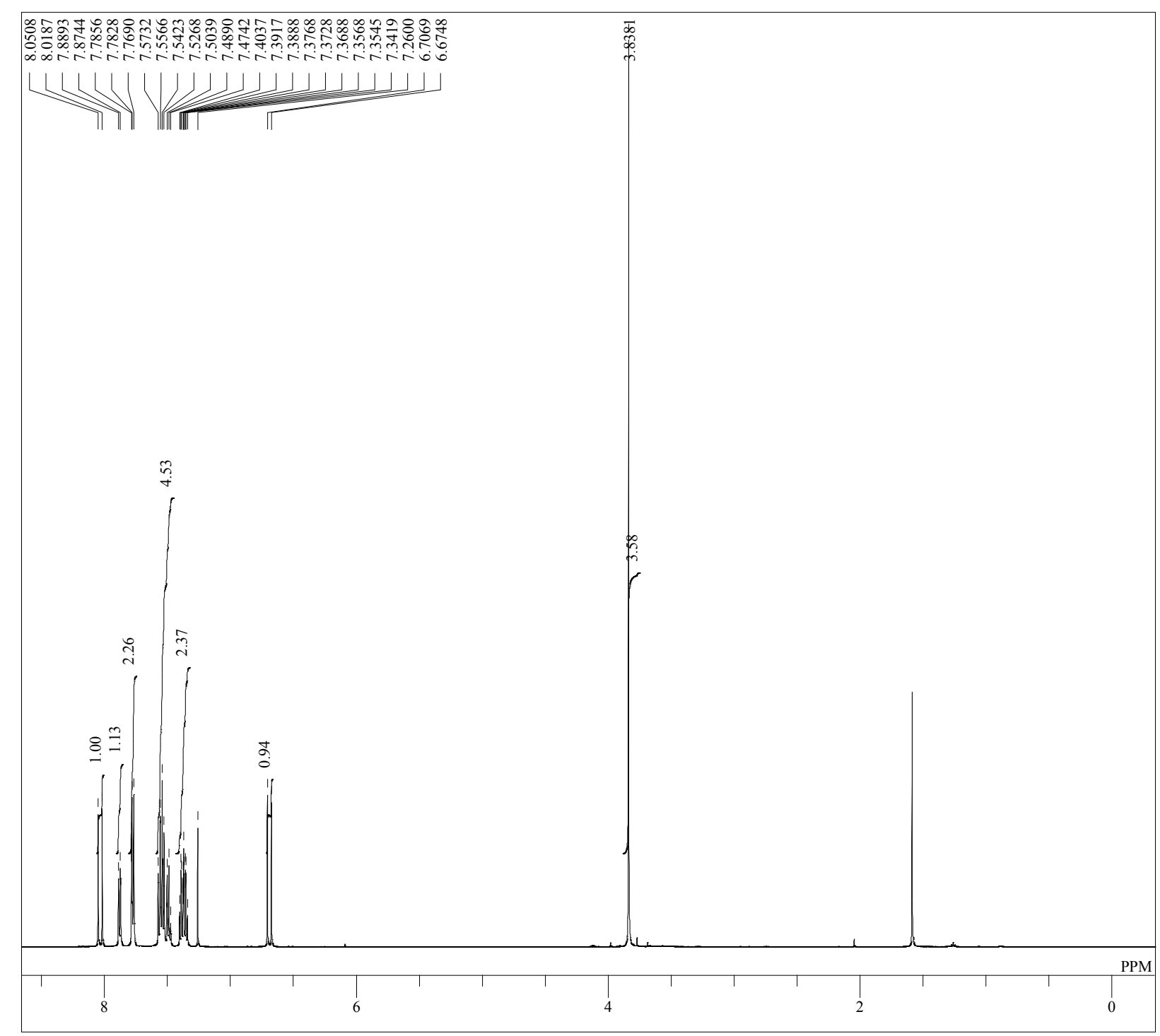

DFILE ph prod_proton-1-1.als

COMNT single pulse

OBNUC $1 \mathrm{H}$

$\begin{array}{ll}\text { EXMOD } & \text { proton.jxp } \\ \text { OBFRQ } & 500.16 \mathrm{MHz}\end{array}$

OBSET $\quad 2.41 \mathrm{KHz}$

POINT 26214

FREQU $\quad 7507.51 \mathrm{~Hz}$

SCANS 16

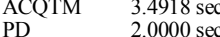

PW1 $\quad 6.50$ usec

$\begin{array}{lll}\text { IRNUC } & 1 \mathrm{H} & 6.50 \\ \text { CTEMP } & 22.8 \mathrm{c}\end{array}$

$\begin{array}{ll}\text { CTEMP } & 22.8 \mathrm{c} \\ \text { SLVNT } & \mathrm{CDCL} 3\end{array}$

$\begin{array}{ll}\text { EXREF } & 7.26 \mathrm{ppm} \\ \mathrm{BF} & 0.12 \mathrm{~Hz}\end{array}$

RGAIN 46

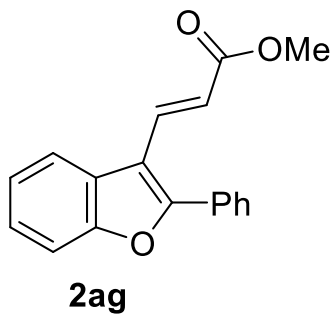

S2 - 92 
single pulse decoupled gated NOE

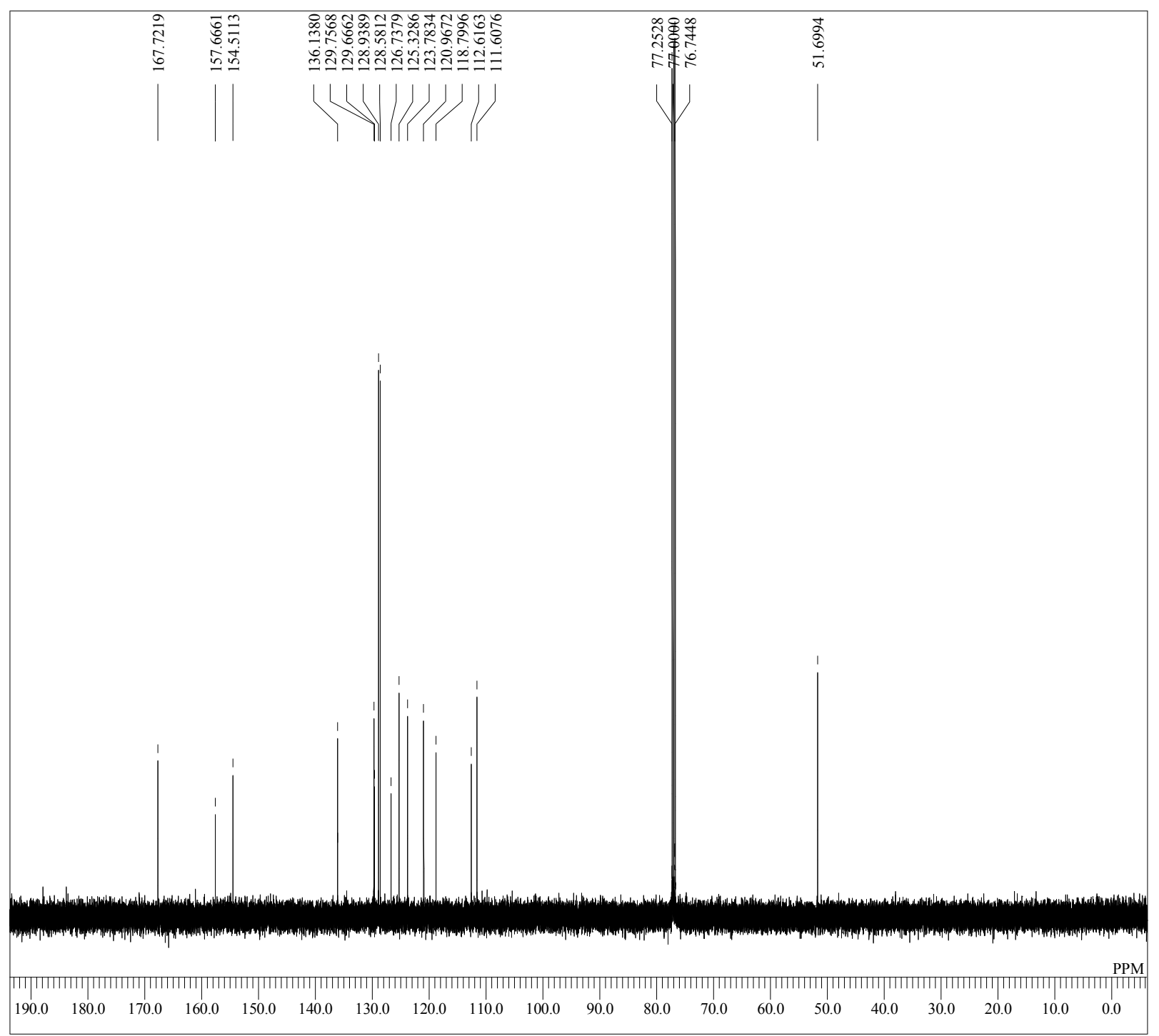

DFILE ph prod Carbon-1-1.als

DOMNT single pulse decoupled gated NOE

OBNUC $13 \mathrm{C}$

EXMOD carbon.jxp

$\begin{array}{ll}104857 \\ \text { POINT } & 104654 \mathrm{~Hz}\end{array}$

SCANS $\quad 96$

ACQTM $\quad 3.3345 \mathrm{sec}$

$\begin{array}{ll}\text { PD } & 1.0000 \mathrm{sec} \\ \text { PW1 } & 3.27 \mathrm{usec}\end{array}$

IRNUC $1 \mathrm{H}$

CTEMP $22.9 \mathrm{c}$

EXREF $77.00 \mathrm{pm}$

$\begin{array}{lc}\text { BF } & 0.12 \mathrm{~Hz} \\ \text { RGAIN } & 58\end{array}$

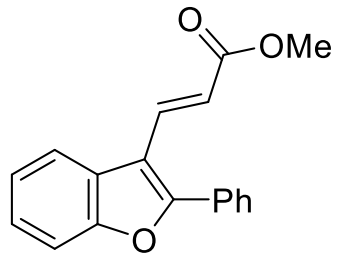

2ag 
single pulse

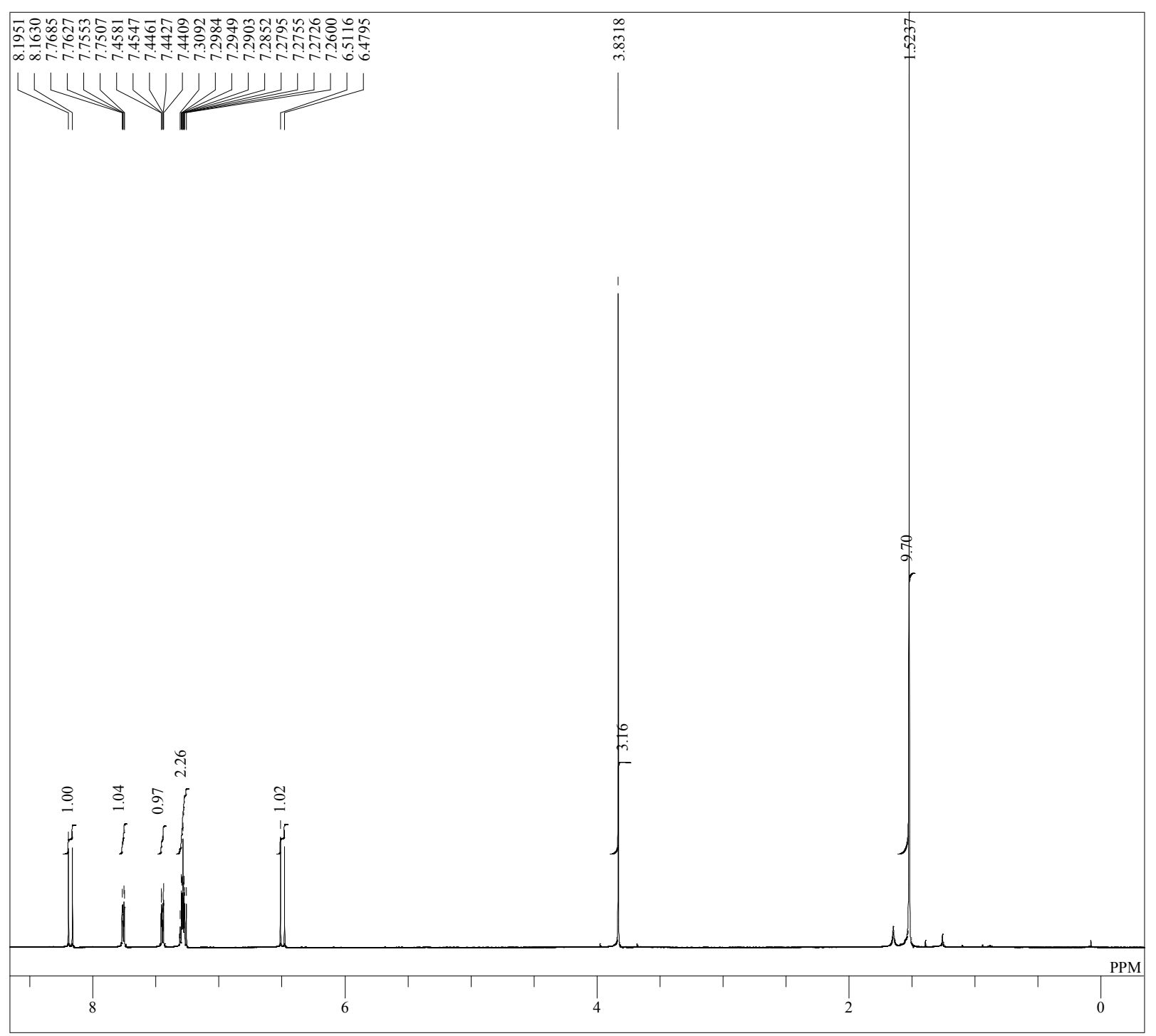

S2 - 94
DFILE tbu prod proton-2-1.als

COMNT single pulse

OBNUC

D proton.jxp

OBFRQ $500.16 \mathrm{MHz}$

OBFIN $\quad 6.01 \mathrm{~Hz}$

$\begin{array}{lll} & 26214 \\ & \text { FREOU } & 7507.51 \mathrm{~Hz}\end{array}$

SCANS $\quad 16$

ACQTM $\quad 3.4918 \mathrm{sec}$

$\begin{array}{ll}\text { PD } & 2.0000 \mathrm{sec} \\ \text { PW1 } & 6.50 \mathrm{usec}\end{array}$

IRNUC 1H

CTEMP $23.3 \mathrm{c}$

EXREF $7.26 \mathrm{ppm}$

$\begin{array}{ll}\text { BX } & 7.26 \mathrm{ppm} \\ \text { RGAIN } & 38\end{array}$

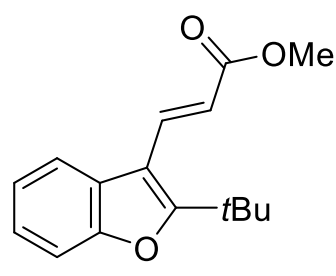

2ah 
single pulse decoupled gated NOE

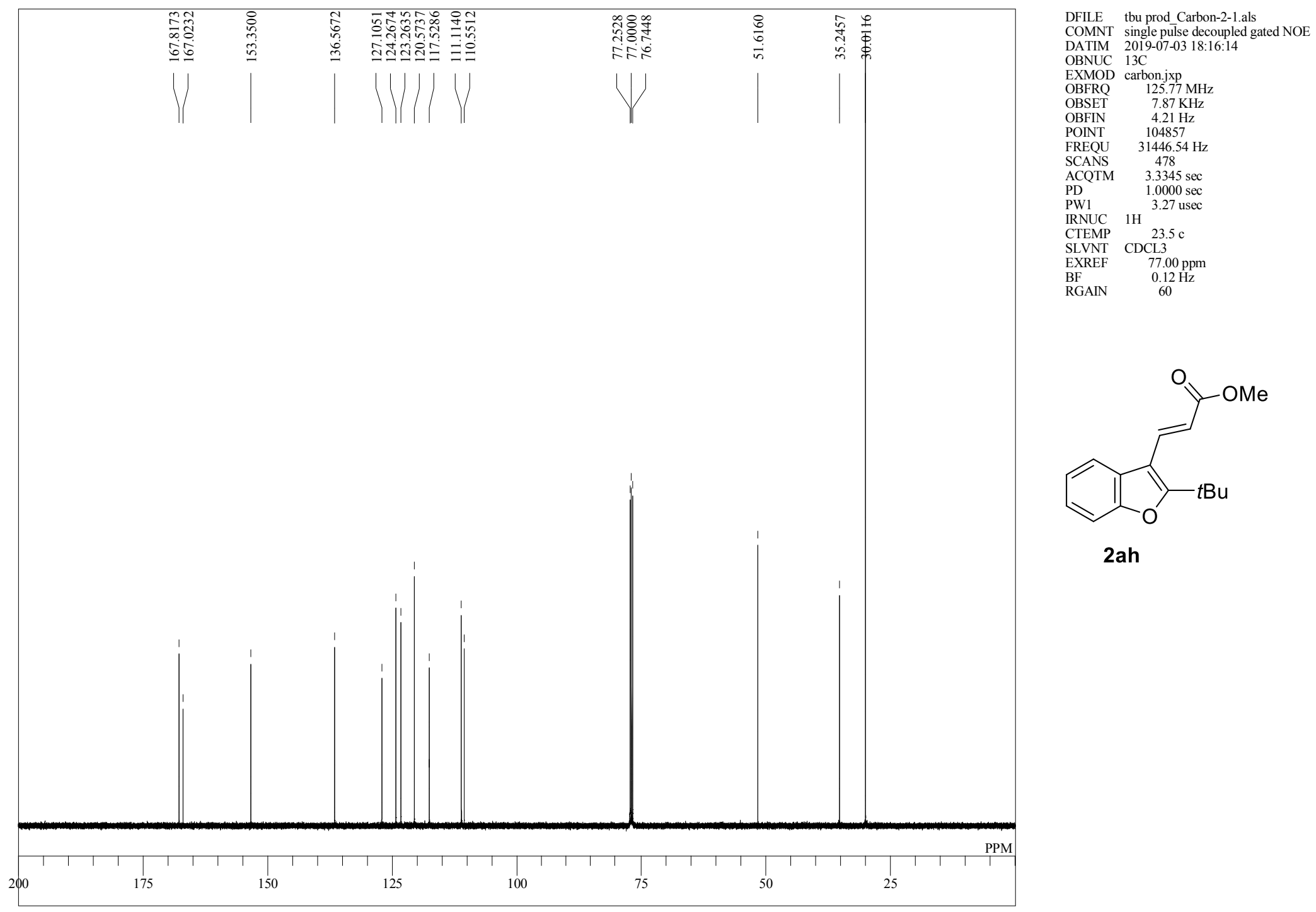


single pulse

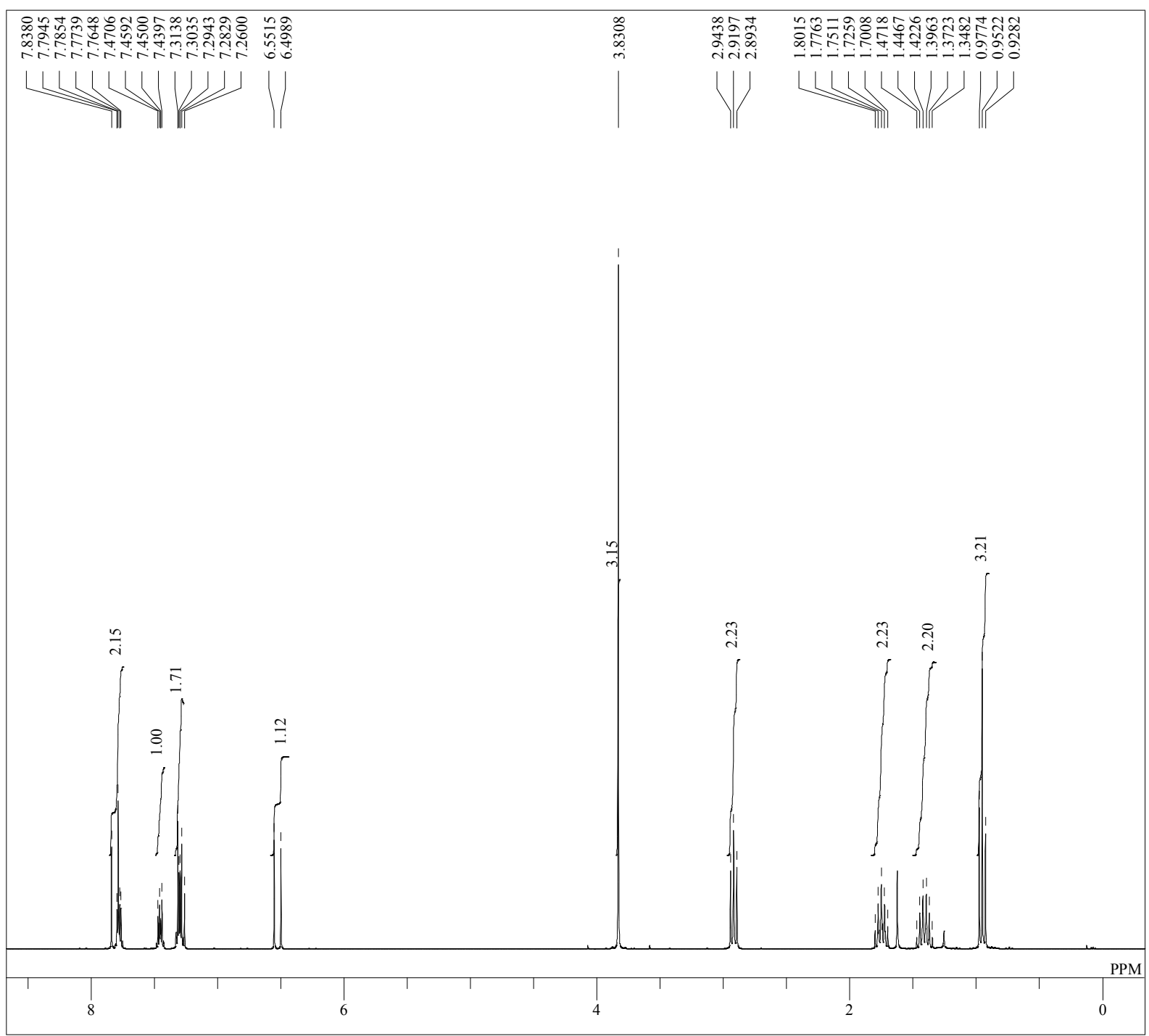

DFILE nbu prod_proton-1-1.als

COMNT single pulse

DXNUC $1 \mathrm{H}$

EXMOD proton.jxp
OBFRQ $300.53 \mathrm{MHz}$

OBSET $\quad 1.15 \mathrm{KHz}$

OBFIN $\quad 8.57 \mathrm{~Hz}$

$\begin{array}{ll} & 13107 \\ \text { FREOU } & 450857 \mathrm{~Hz}\end{array}$

$\begin{array}{lc}\text { SCANS } & 16 \\ \text { ACQTM } & 2.9072 \mathrm{sec}\end{array}$

$\begin{array}{ll}\text { PD } & 2.0000 \mathrm{sec} \\ \text { PW1 } & 5.50 \mathrm{usec}\end{array}$

IRNUC $1 \mathrm{H}$

CTEMP $23.1 \mathrm{c}$

$\begin{array}{lc}\text { SLVNT } & \text { CDCL3 } \\ \text { EXREF } & 7.26 \mathrm{ppm} \\ \mathrm{BF} & 0.12 \mathrm{~Hz}\end{array}$

RGAIN $\quad 36$

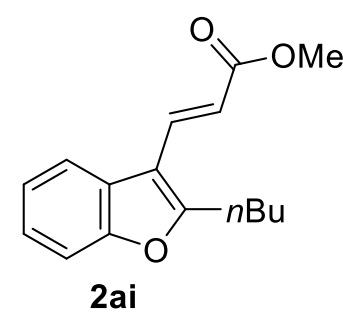

S2 - 96 
single pulse decoupled gated NOE

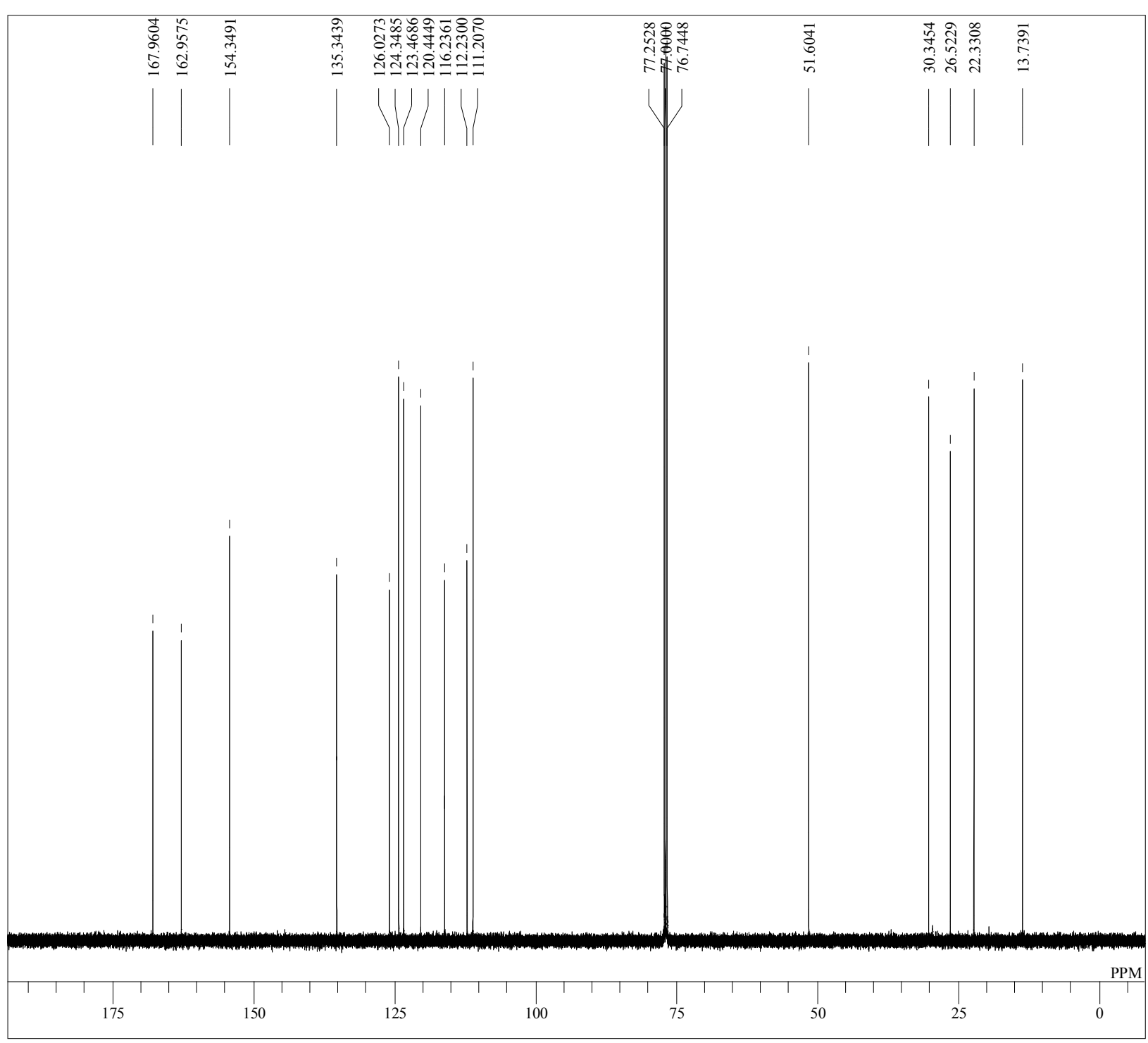

DFILE nbu prod_Carbon-1-1.als

gated $\mathrm{NOE}$

OBNUC $13 \mathrm{C}$

$\begin{array}{lll}\text { EXMOD } & \text { carbon.jxp } \\ \text { OBFRQ } & 125.77 \mathrm{MHz}\end{array}$

OBSET $\quad 7.87 \mathrm{KHz}$

POINT 104857

FREQU $\quad 31446.54 \mathrm{~Hz}$

$\begin{array}{lll}6 & 692 \\ \text { SCANS } & 69345\end{array}$

$\begin{array}{ll}\text { PD } & 3.3345 \mathrm{sec} \\ 1.0000 \mathrm{sec}\end{array}$

PW1 3.27 use

$\begin{array}{lll}\text { IRNUC } & 1 \mathrm{H} & \\ \text { CTEMP } & & 23.4 \mathrm{c}\end{array}$

SLVNT CDCL3

EXREF $\quad 77.00 \mathrm{ppn}$

RGAIN

$0.12 \mathrm{~Hz}$

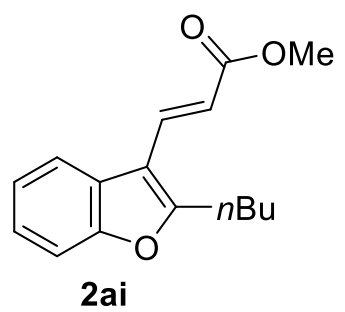

2ai 
single pulse

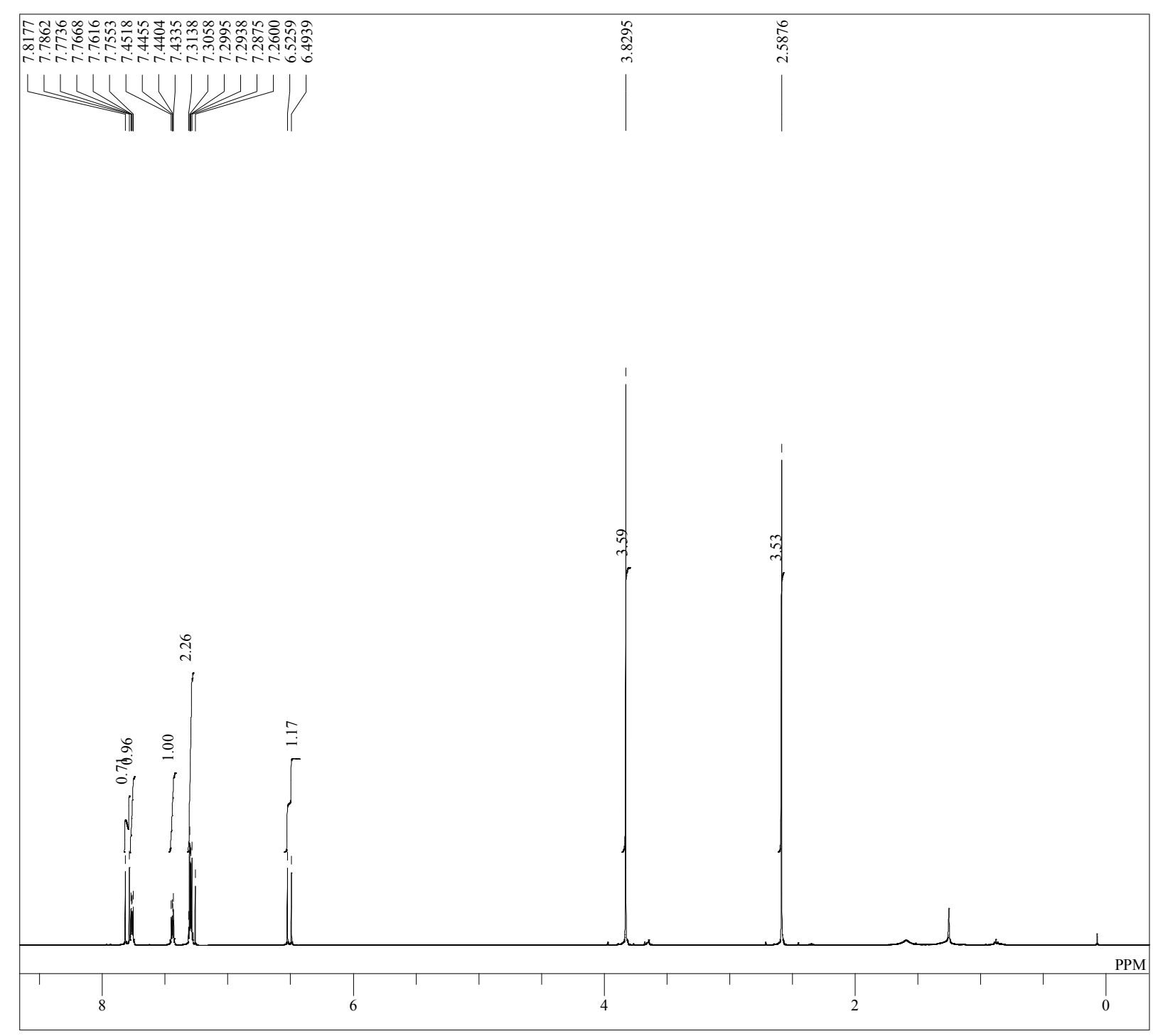

DFILE me prod_proton-1-1.als

COMNT single pulse

OBNUC $1 \mathrm{H}$

EXMOD pro

OBFRQ $\quad 500.16 \mathrm{MHz}$

OBSET $2.41 \mathrm{KHz}$

$\begin{array}{ll}\text { OBFIN } & 6.01 \mathrm{~Hz} \\ \text { POINT } & 26214\end{array}$

FREQU $\quad 7507.51 \mathrm{~Hz}$

SCANS $\quad 16$

$\begin{array}{ll}\text { ACQTM } & 3.4918 \mathrm{sec}\end{array}$

$\begin{array}{ll} & 2.0000 \mathrm{sec} \\ \text { PW1 } & 6.50 \mathrm{usec}\end{array}$

IRNUC $1 \mathrm{H}$

CTEMP $23.2 \mathrm{c}$

EXREF $7.26 \mathrm{ppm}$

$\begin{array}{lc}\text { BF } & 0.12 \mathrm{~Hz} \\ \text { RGAIN } & 48\end{array}$

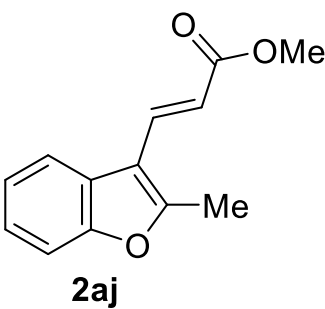

S2- 98 
single pulse decoupled gated NOE

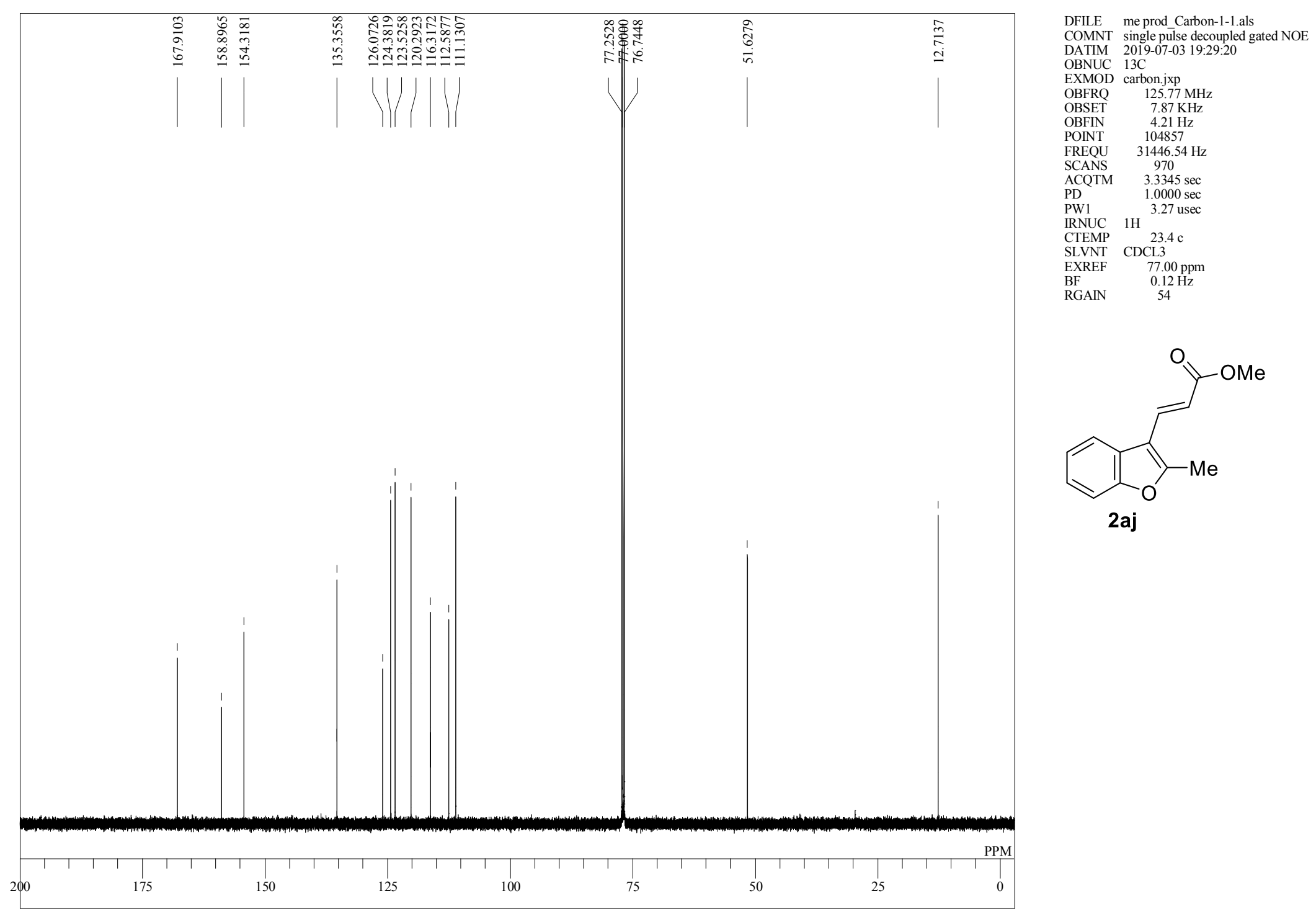

S2 - 99 
single pulse

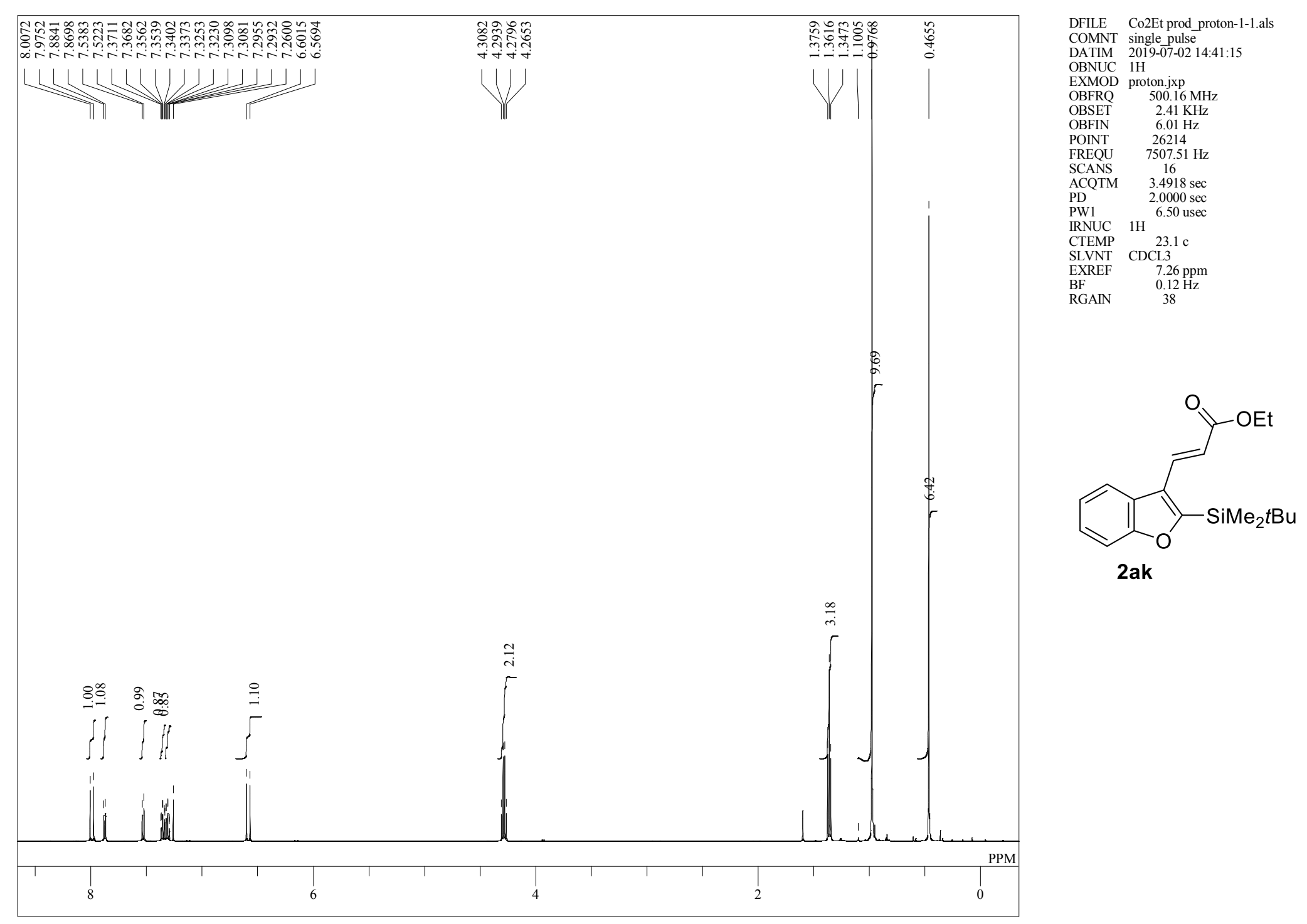

S2 - 100 
single pulse decoupled gated NOE

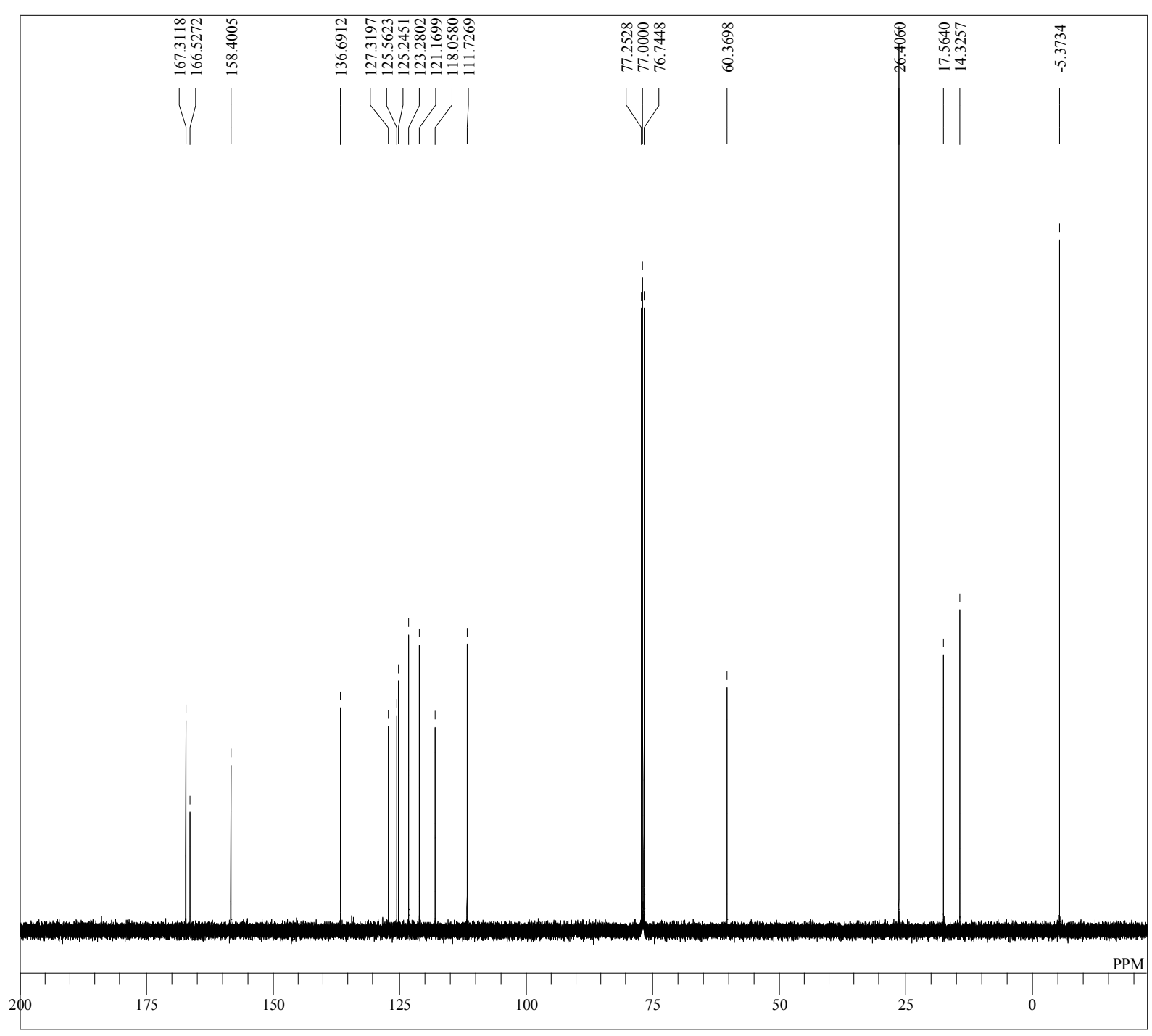

DFILE Co2Et prod_Carbon-1-1.als

DATIM $2019-07-02 \quad 14 \cdot 22: 18$

OBNUC 13C

OBFRQ $125.77 \mathrm{MHz}$

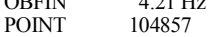

FREQU $\quad 31446.54 \mathrm{~Hz}$

$\begin{array}{ll}\text { SCANS } & 250 \\ \text { ACQTM } & 3.3345 \mathrm{sec}\end{array}$

$\begin{array}{lll}\mathrm{PD} & 1.0000 \mathrm{sec}\end{array}$

IRNUC $1 \mathrm{H}$

$\begin{array}{ll}\text { CTEMP } & 23.4 \mathrm{c} \\ \text { SLVNT } & \text { CDCL3 }\end{array}$

$\begin{array}{ll}\text { SLVNT } & \text { CDCL3 } \\ \text { EXREF } & 77.00 \mathrm{ppm}\end{array}$

$\begin{array}{lc}\text { EXREF } & 77.00 \mathrm{ppm} \\ \text { BF } & 0.12 \mathrm{~Hz} \\ \text { RGAIN } & 60\end{array}$

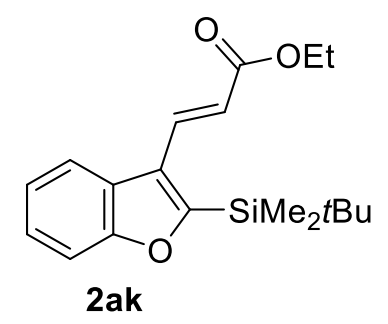

S2 - 101 
single _pulse

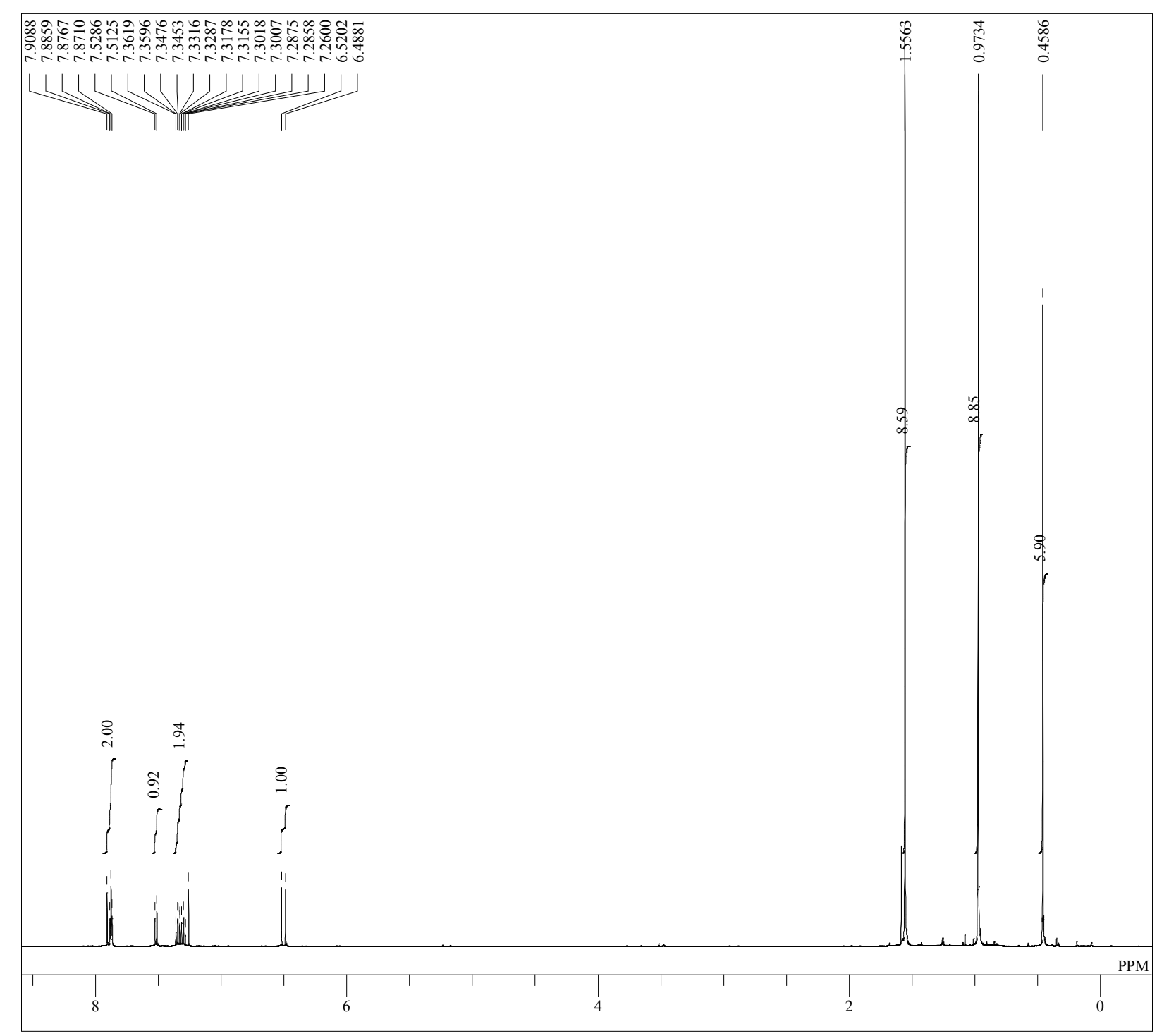

DFILE co2tbu prod_proton-1-1.als

DATIM 2019-07-04 17:24:54

OBNUC $1 \mathrm{H}$

EXMOD

OBFRQ $\quad 500.16 \mathrm{MHz}$

OBSET $\quad 2.41 \mathrm{KHz}$

POINT 26214

FREQU $\quad 7507.51 \mathrm{~Hz}$

$\begin{array}{ll}\text { SCANS } & 16 \\ \text { ACOTM } & 3.4918 \mathrm{sec}\end{array}$

$\begin{array}{ll}\mathrm{PD} & 3.4918 \mathrm{sec} \\ & 20000 \mathrm{sec}\end{array}$

\begin{tabular}{ll} 
PW1 & $2.5000 \mathrm{sec}$ \\
\hline RNUC & $6.9 \mathrm{c}$
\end{tabular}

IRNUC $1 \mathrm{H} 21.9 \mathrm{c}$

$\begin{array}{ll}\text { CTEMP } & 21.9 \\ \text { SLVNT } & \text { CDCL } 3\end{array}$

$\begin{array}{ll}\text { EXREF } & 7.26 \mathrm{ppm} \\ \mathrm{BF} & 0.12 \mathrm{~Hz}\end{array}$

RGAIN $\quad 42$

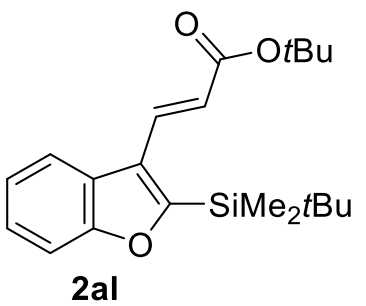

S2 - 102 
single pulse decoupled gated NOE

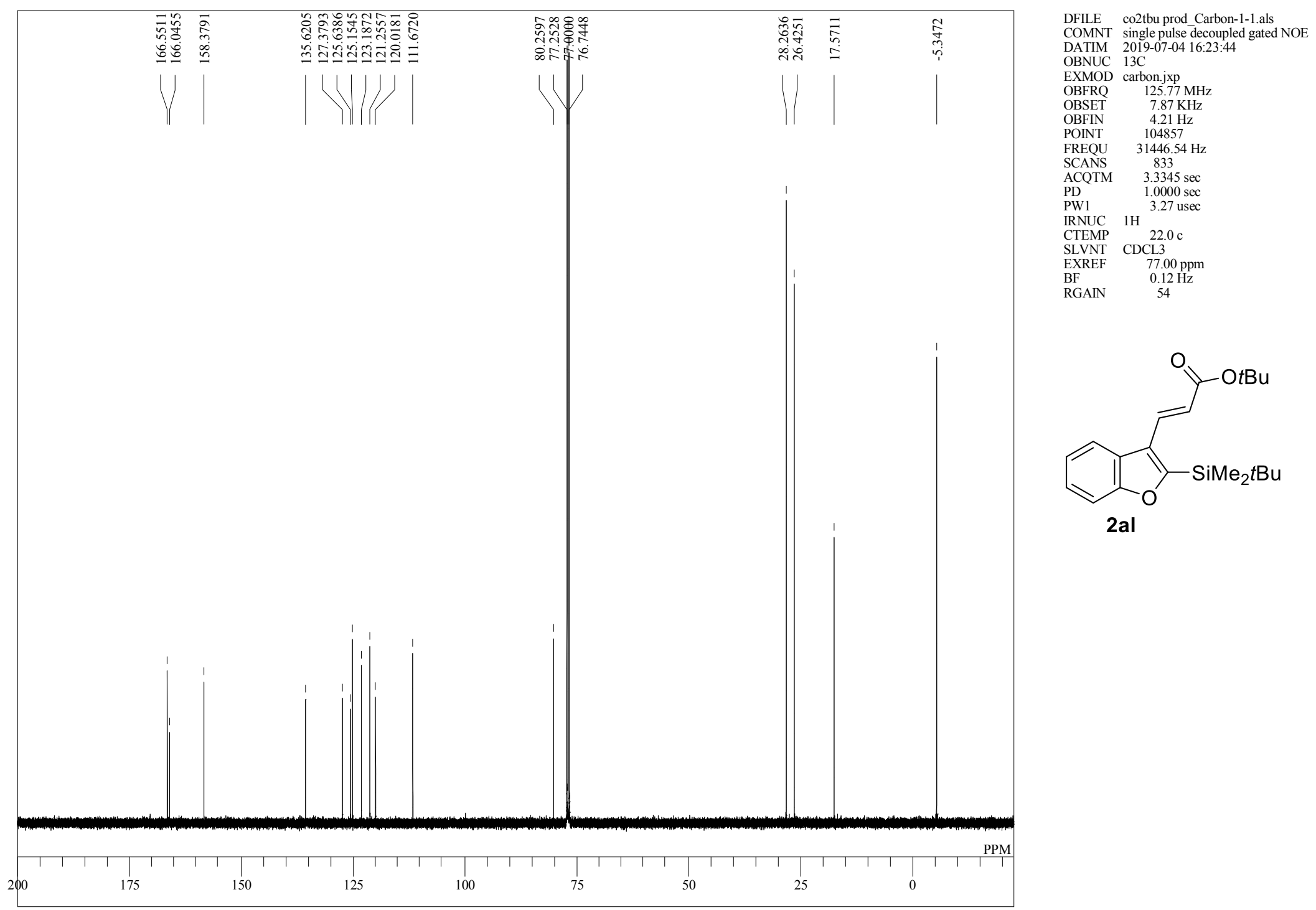

S2- 103 
single pulse

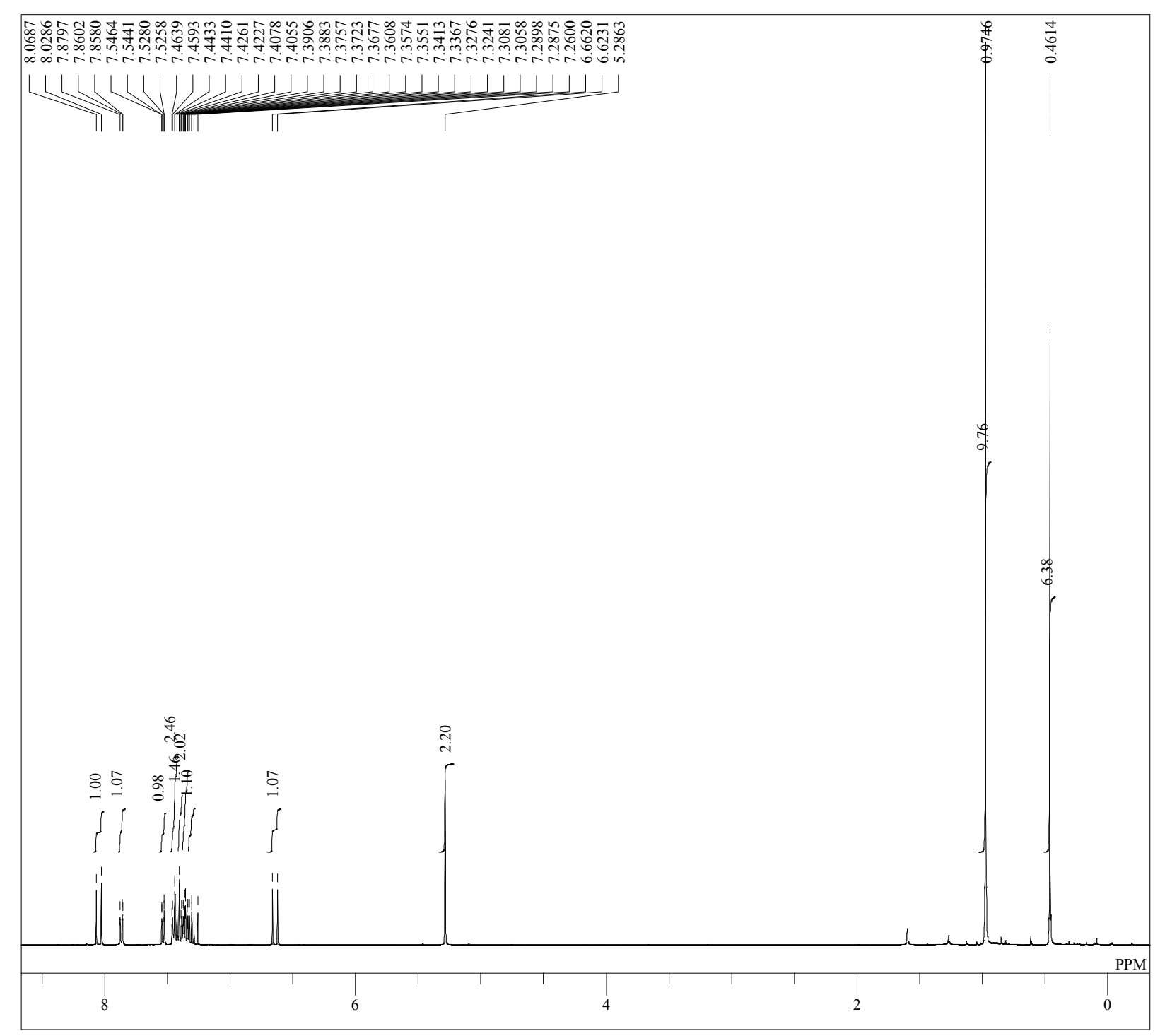

DFILE co2bn prod_proton-1-1.als

$\begin{array}{ll}\text { COMNT } & \text { single pulse } \\ \text { DATIM } & 2019-06-28 \text { 23:13:54 }\end{array}$

OBNUC $1 \mathrm{H}$

EXMOD proton.jxp
OBFRQ $399.78 \mathrm{MH}$

OBSET $\quad 4.19 \mathrm{KHz}$

$\begin{array}{ll}\text { OBFIN } & 7.29 \mathrm{~Hz} \\ \text { POINT } & 13107\end{array}$

FREQU $\quad 6002.40 \mathrm{~Hz}$

SCANS $\quad 16$

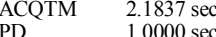

$\begin{array}{ll}\text { PD } & 1.0000 \mathrm{sec} \\ \text { PW1 } & 7.25 \mathrm{usec}\end{array}$

IRNUC $1 \mathrm{H}$

CTEMP $22.6 \mathrm{c}$

EXREF $7.26 \mathrm{ppm}$

$\begin{array}{ll}\text { BF } & 0.12 \mathrm{~Hz} \\ \text { RGAIN } & 36\end{array}$

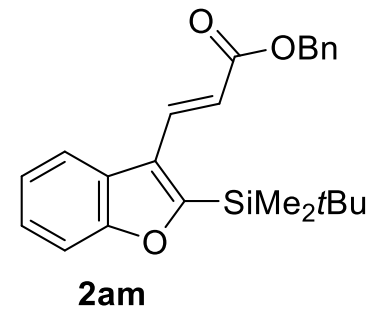

2am 
single pulse decoupled gated NOE

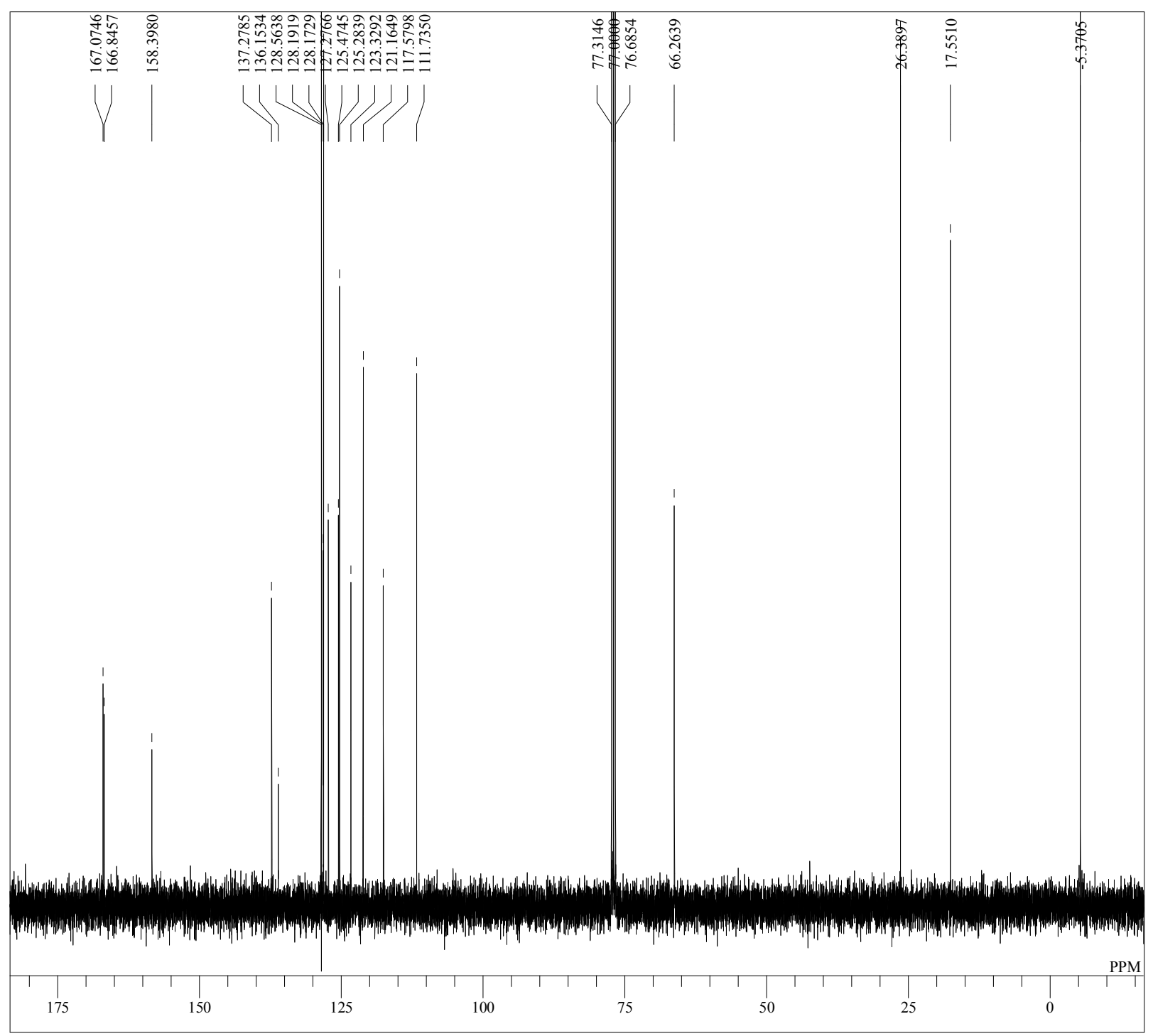

DFILE co2bn prod_carbon-1-1.als

COMNT single pulse decoupled

BNUC 13C

$\begin{array}{lc}\text { EXMOD } & \text { carbon.jxp } \\ \text { OBFRQ } & 100.53 \mathrm{MHz} \\ \text { OBSET } & 5.35 \mathrm{KHz}\end{array}$

OBFIN $\quad 5.86 \mathrm{~Hz}$

FREQU $25125.63 \mathrm{~Hz}$

SCANS 141

$\begin{array}{ll}\text { ACQTM } & 1.0433 \mathrm{sec} \\ \mathrm{PD} & 2.0000 \mathrm{sec}\end{array}$

$\begin{array}{ll}\mathrm{PD} & 2.0000 \mathrm{sec} \\ \mathrm{PW} 1 & 3.17 \mathrm{usec}\end{array}$

IRNUC $1 \mathrm{H} 22 \mathrm{c}$

$\begin{array}{ll}\text { CTEMP } & 22.9 \mathrm{c} \\ \text { SLVNT } & \text { CDCL3 }\end{array}$

EXREF $\quad 77.00 \mathrm{ppm}$

$\begin{array}{lc}\text { BF } & 0.12 \mathrm{~Hz} \\ \text { RGAIN } & 60\end{array}$

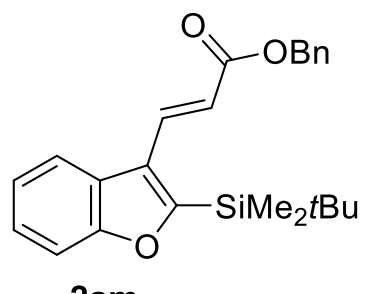

2am 
single pulse

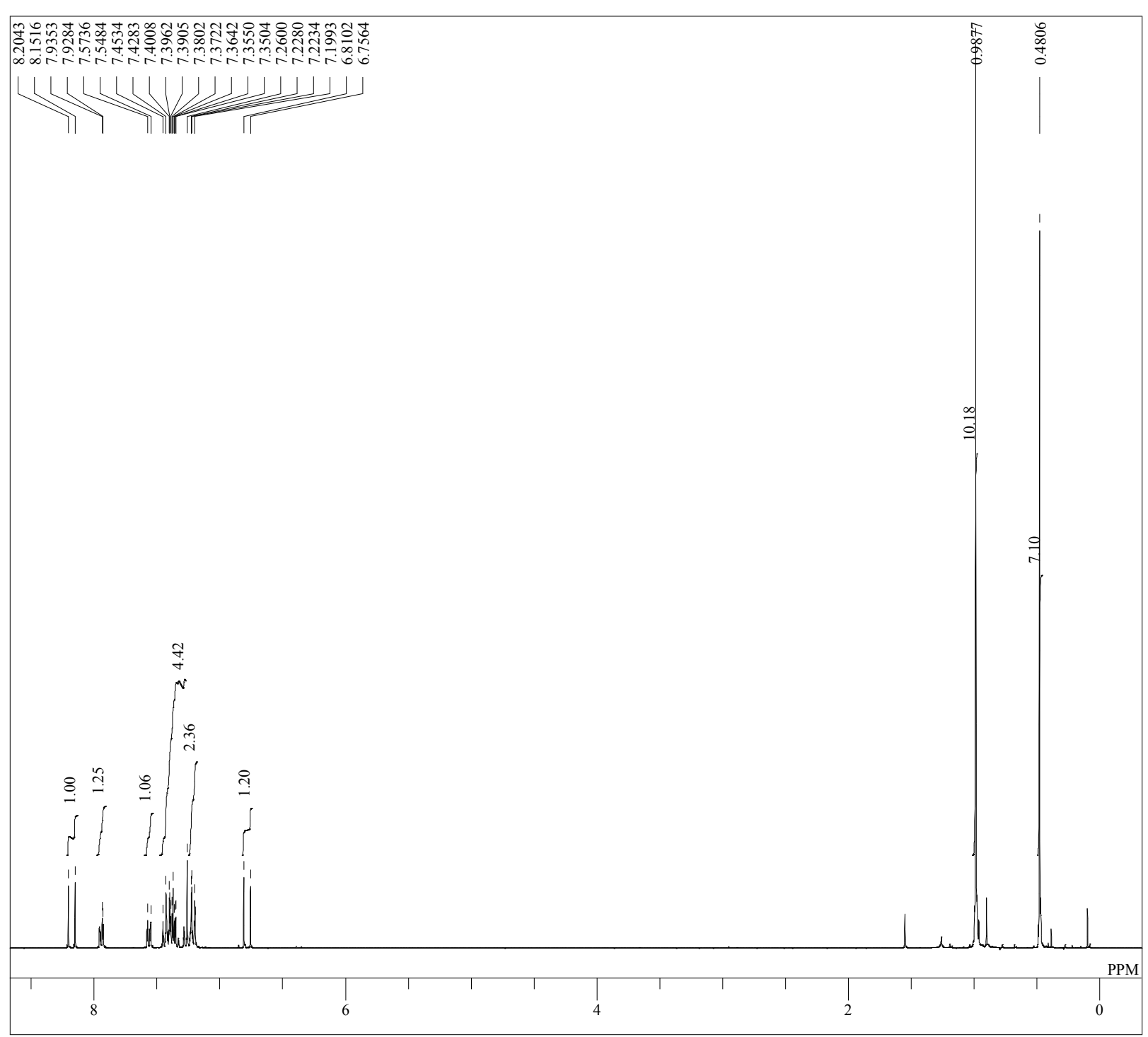

DFILE co2ph prod_proton-1-1.als

COMNT single pulse 103.33

$\begin{array}{ll}\text { EXMOD } & \text { proton.jxp } \\ \text { OBFRQ } & 300.53 \mathrm{MHz}\end{array}$

OBSET $\quad 1.15 \mathrm{KHz}$

$\begin{array}{ll}\text { OBFIN } & 8.57 \mathrm{~Hz} \\ \text { POINT } & 13107\end{array}$

FREQU $\quad 4508.57 \mathrm{~Hz}$

$\begin{array}{lll}\text { SCANS } & 8 \\ \text { ACQTM } & 29072\end{array}$

$2.9072 \mathrm{sec}$
$\mathrm{PD}$

\begin{tabular}{ll} 
PW1 & $2.0000 \mathrm{sec}$ \\
\hline & $5.50 \mathrm{usec}$
\end{tabular}

IRNUC 1H

CTEMP $23.3{ }^{\circ}$

$\begin{array}{ll}\text { EXREF } & 7.26 \mathrm{ppm} \\ \mathrm{BF} & 0.12 \mathrm{~Hz}\end{array}$

$\begin{array}{ll}\text { BF } & 0.12 \mathrm{~Hz} \\ \text { RGAIN } & 38\end{array}$

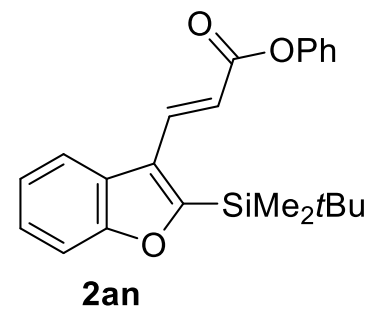

S2- 106 
single pulse decoupled gated NOE

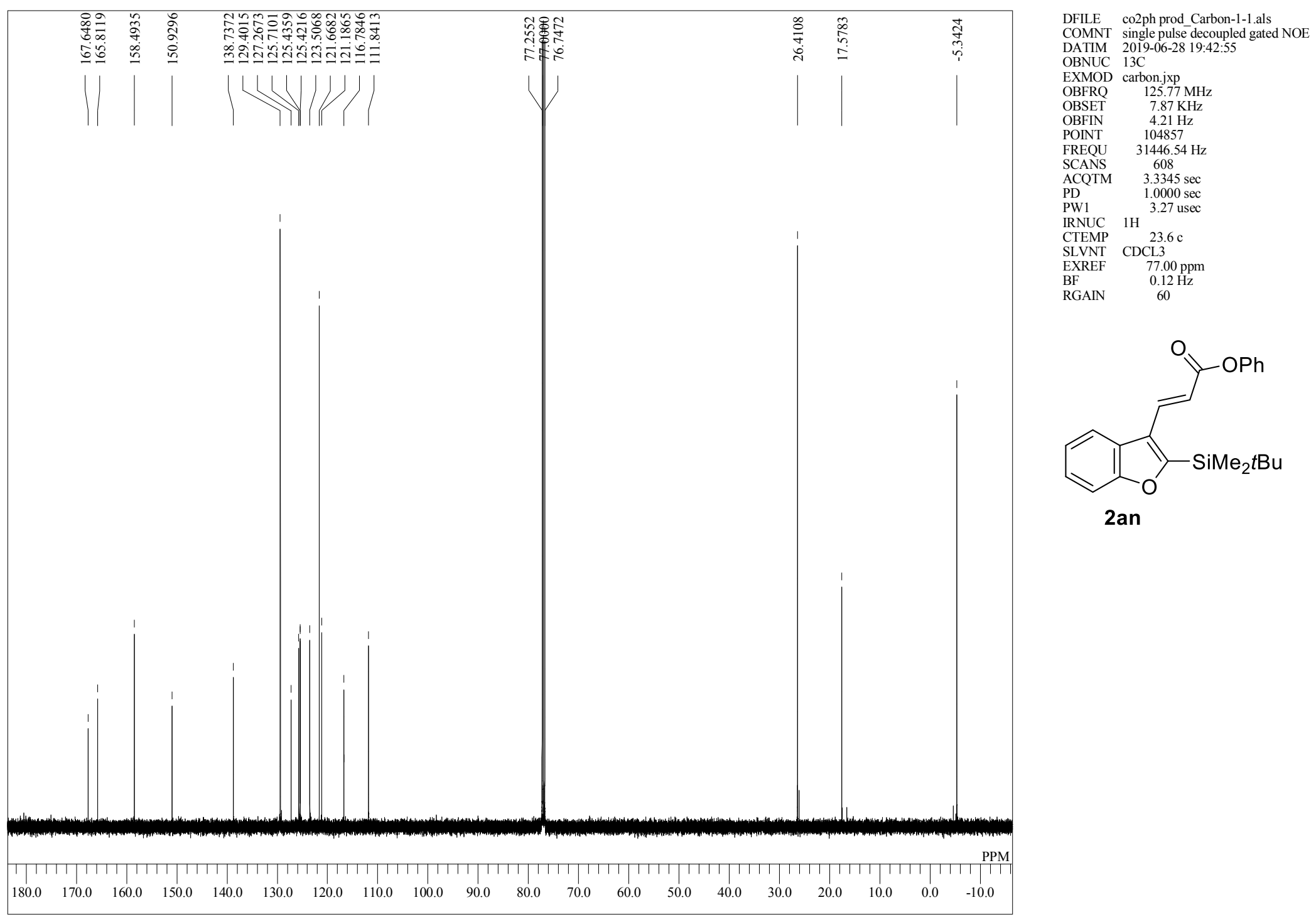

S2- 107 
single pulse

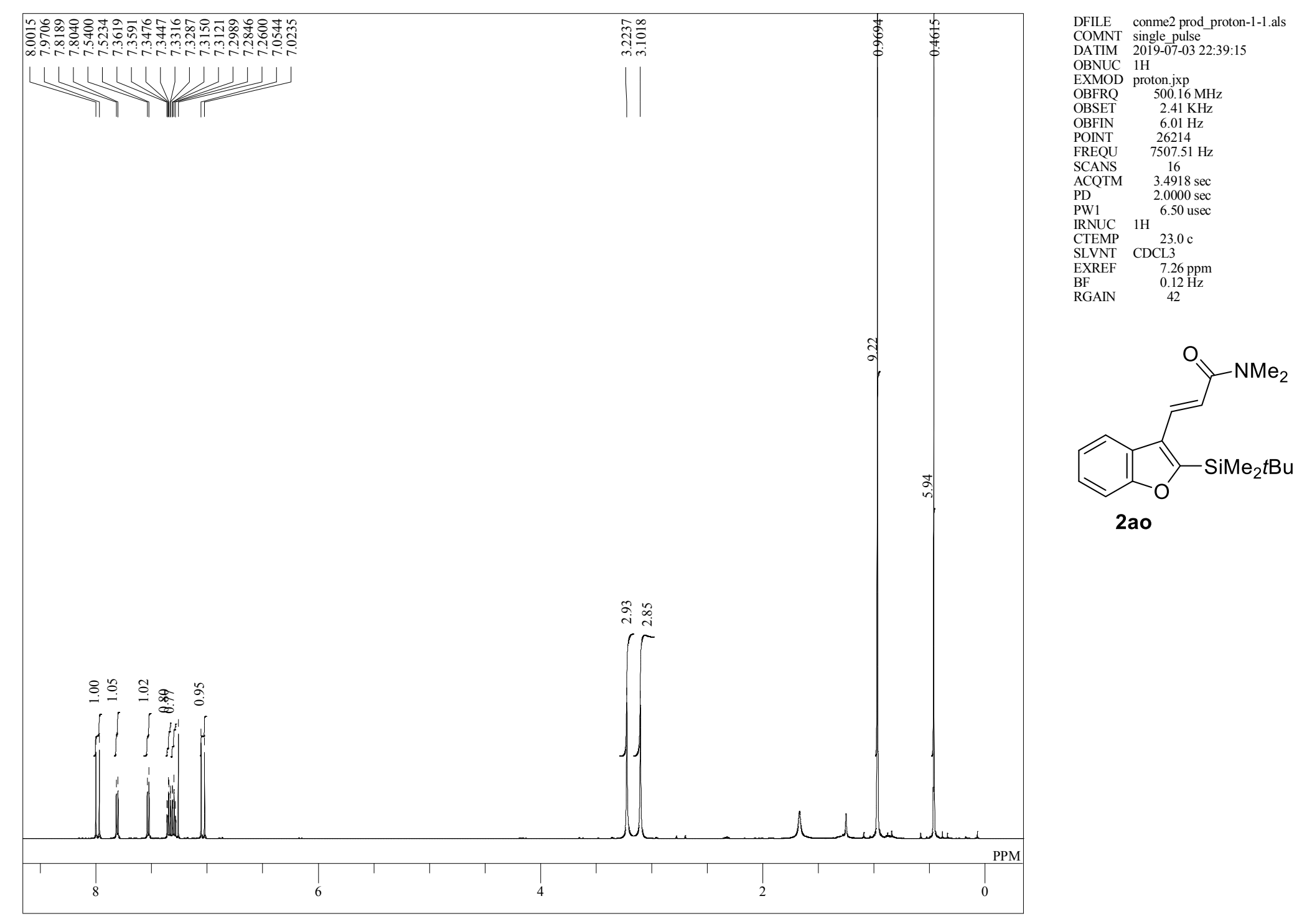

S2 - 108 
single pulse decoupled gated NOE

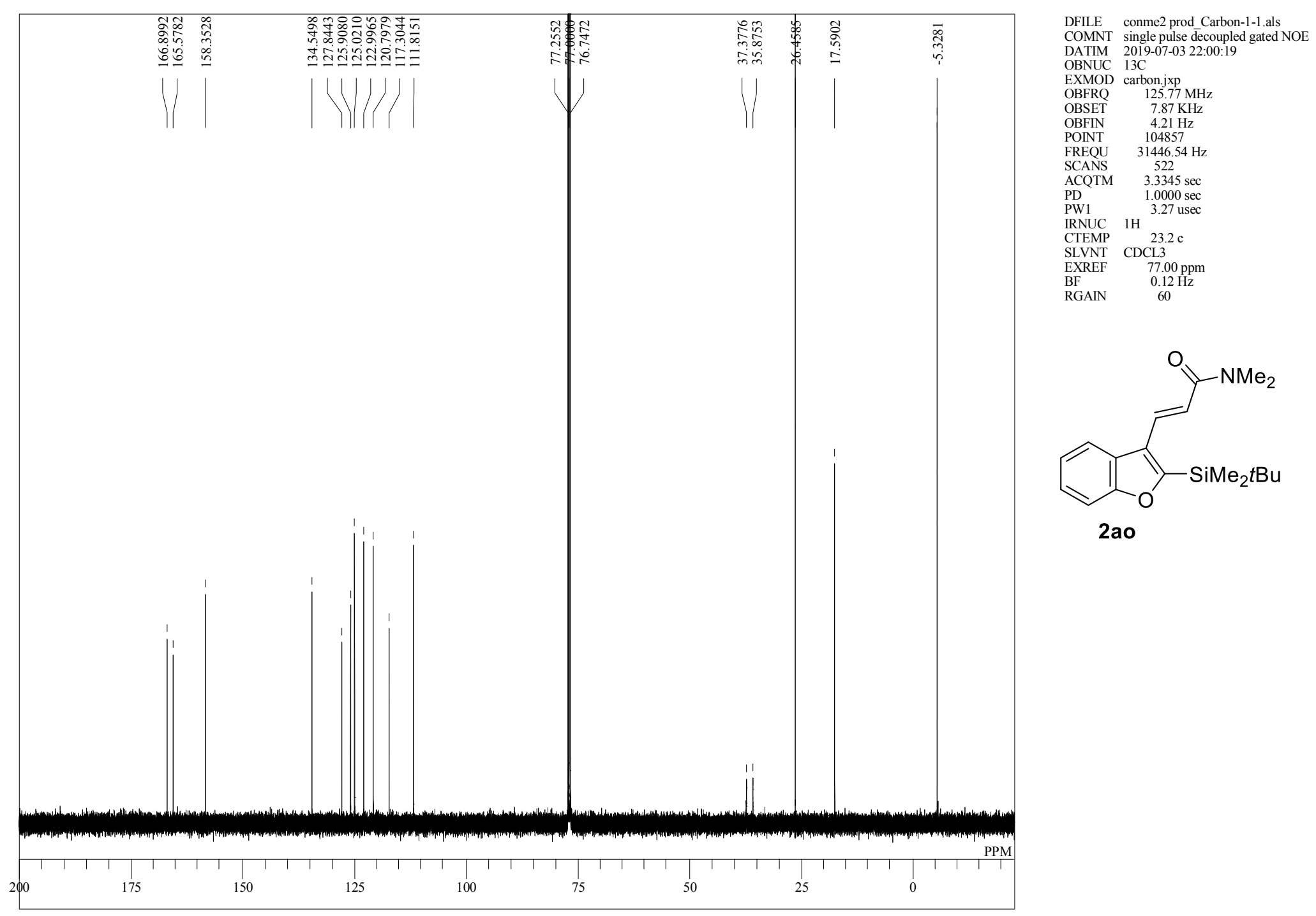

$\mathrm{S} 2-109$ 
single pulse

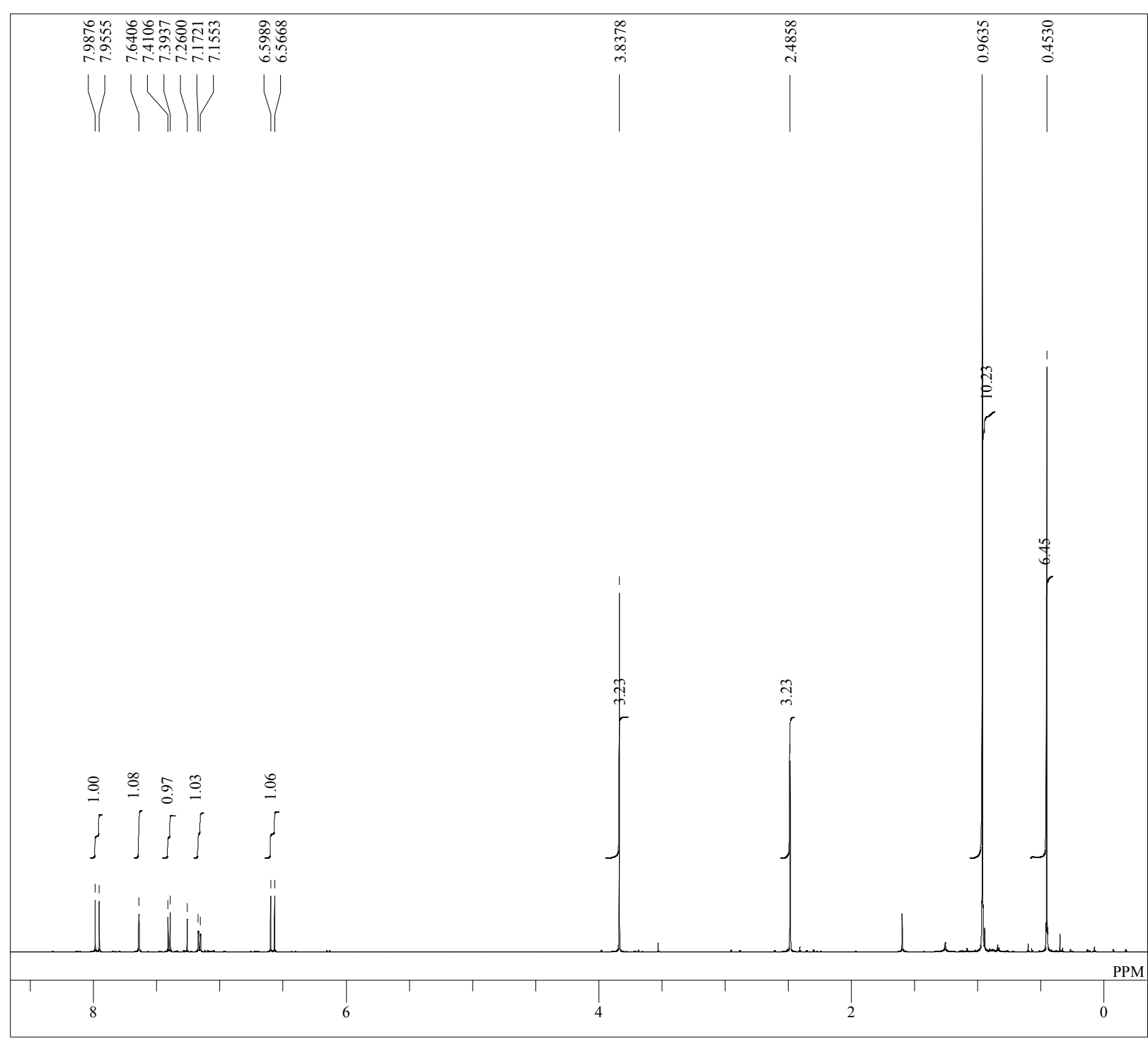

DFILE 4-me prod_proton-1-1.als

$\begin{array}{ll}\text { COMNT } & \text { single pulse } \\ \text { DATIM } & \text { 2019-06-27 21:44:19 }\end{array}$

OBNUC $1 \mathrm{H}$

$\begin{array}{ll}\text { EXMOD } & \text { proton.jxp } \\ \text { OBFRQ } & 500.16 \mathrm{MHz}\end{array}$

$\begin{array}{ll}\text { OBSET } & 2.41 \mathrm{KHz} \\ \text { OBFIN } & 6.01 \mathrm{~Hz}\end{array}$

POINT 26214

FREQU $\quad 10020.04 \mathrm{~Hz}$

16
SCANS

$\begin{array}{ll}\mathrm{PD} & 2.6162 \mathrm{sec} \\ & \end{array}$

PW1 6.50 usec

IRNUC 1H

$\begin{array}{lr}\text { CTEMP } & 22.8 \mathrm{c} \\ \text { SLVNT } & \text { CDCL3 }\end{array}$

$\begin{array}{lc}\text { EXREF } & 7.26 \mathrm{ppm} \\ \text { BF } & 0.12 \mathrm{~Hz}\end{array}$

RGA

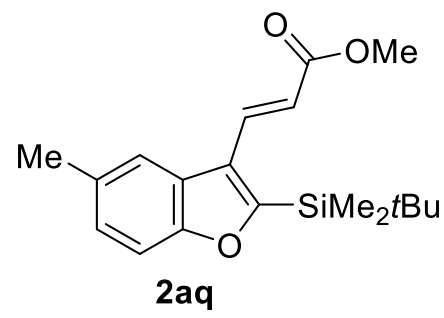

S2 - 110 
single pulse decoupled gated NOE

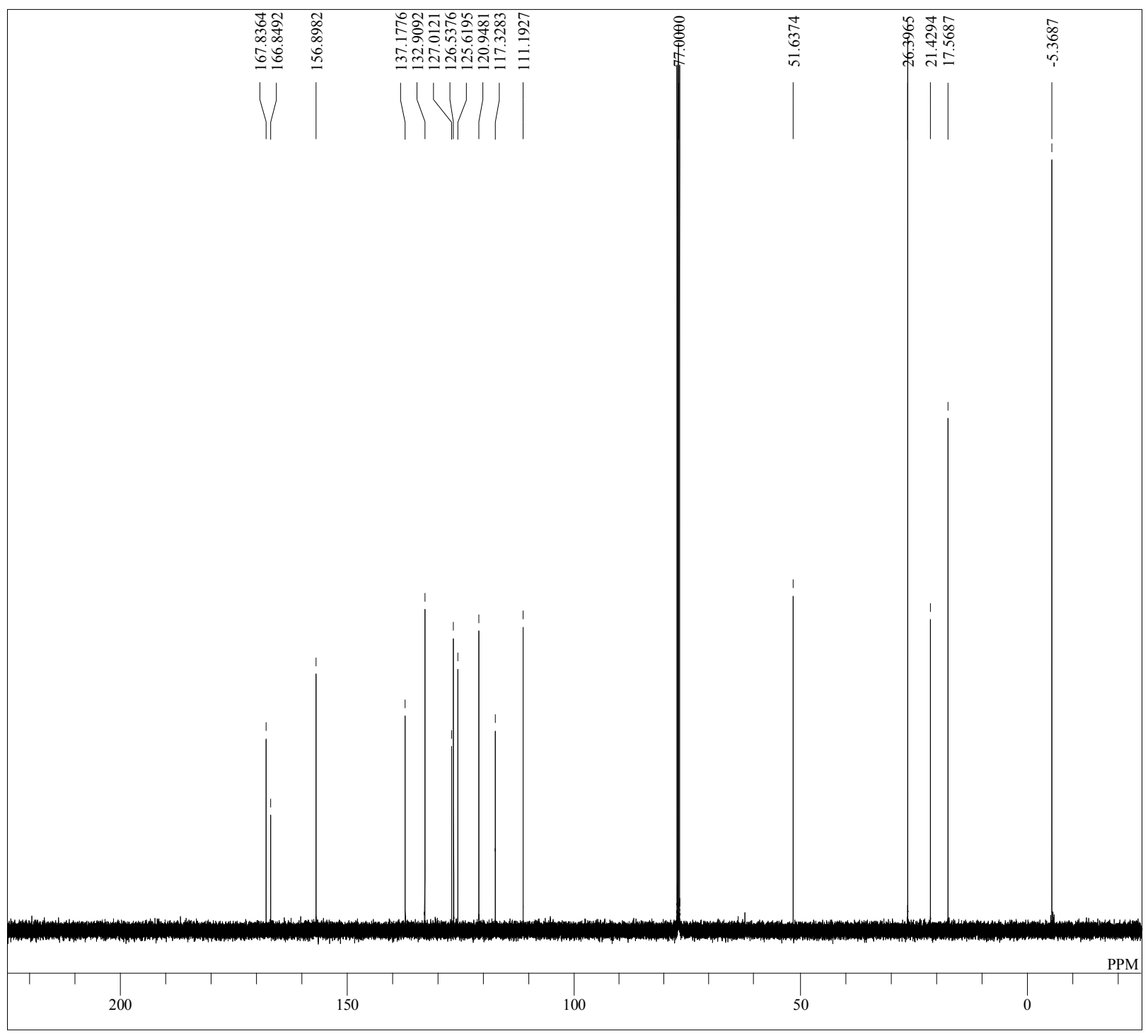

$\begin{array}{ll}\text { DFILE } & \text { 4-me prod Carbon-1-1.als } \\ \text { COMNT } & \text { single pulse decoupled gated NOE }\end{array}$

COMNT single pulse decoupled gated NOE

EXMOD carbon.jxp
OBFRO 12577 M

OBFRQ $\quad 125.77 \mathrm{MHz}$

$\begin{array}{ll}\text { OBSET } & 7.87 \mathrm{KH} z \\ \text { OBFIN } & 4.21 \mathrm{~Hz}\end{array}$

POINT 104857

FREQU $\quad 31446.54 \mathrm{~Hz}$

$\begin{array}{ll}\text { SCANS } & 331 \\ \text { ACQTM } & 3.3345 \mathrm{sec}\end{array}$

$\mathrm{PD} \quad 1.0000 \mathrm{sec}$

PW1 $\quad 3.27$ usec

IRNUC $1 \mathrm{H} 23.0 \mathrm{c}$

$\begin{array}{ll}\text { CTEMP } & 23.0 \mathrm{c} \\ \text { SLVNT } & \text { CDCL3 }\end{array}$

$\begin{array}{ll}\text { EXREF } & 77.00 \mathrm{ppm} \\ \mathrm{BF} & 0.12 \mathrm{~Hz}\end{array}$

RGA

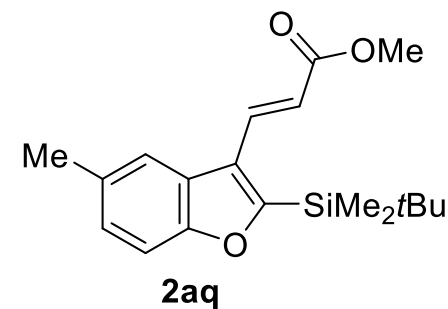

$\mathrm{S} 2-111$ 
single pulse

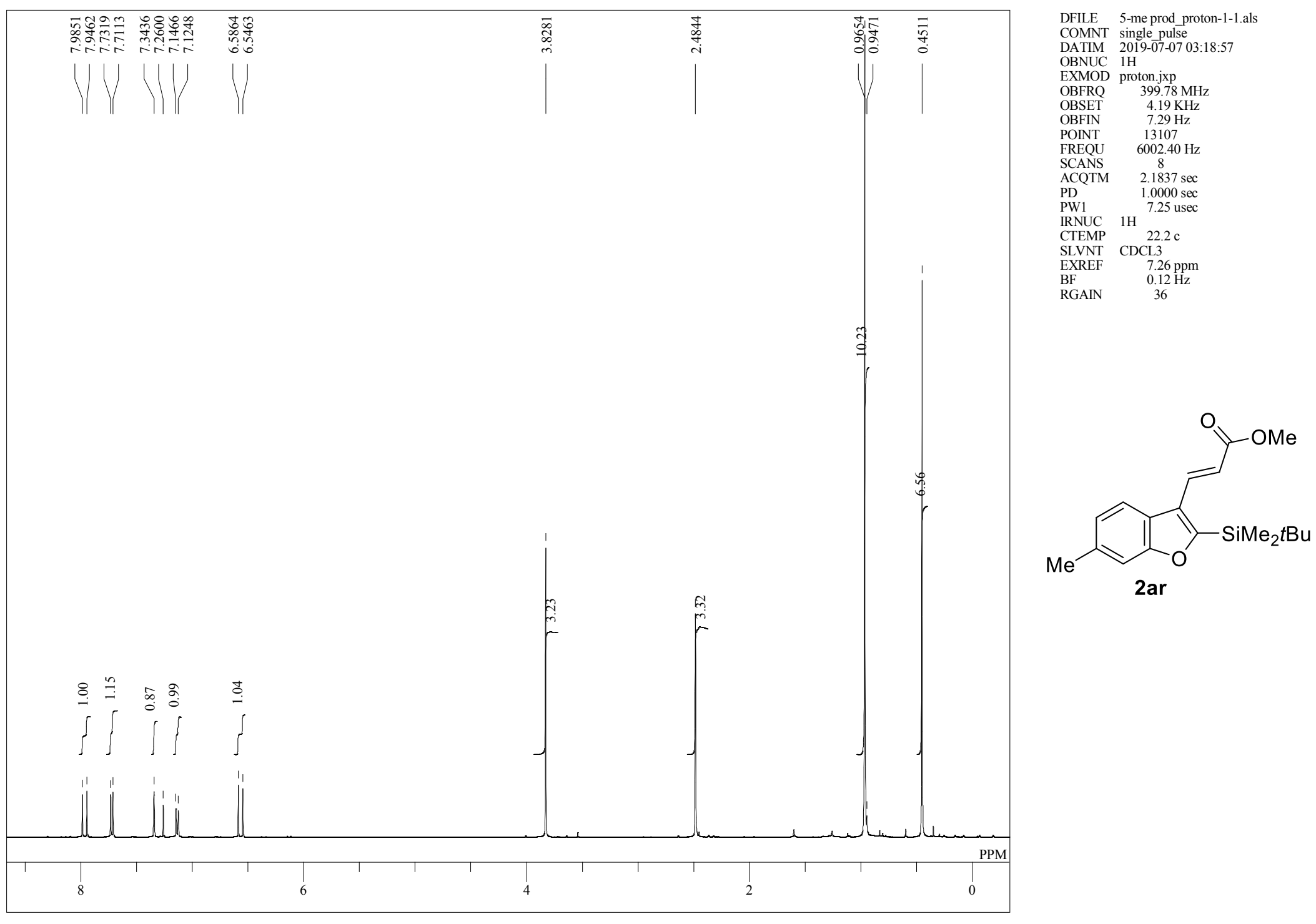

S2 - 112 
single pulse decoupled gated NOE

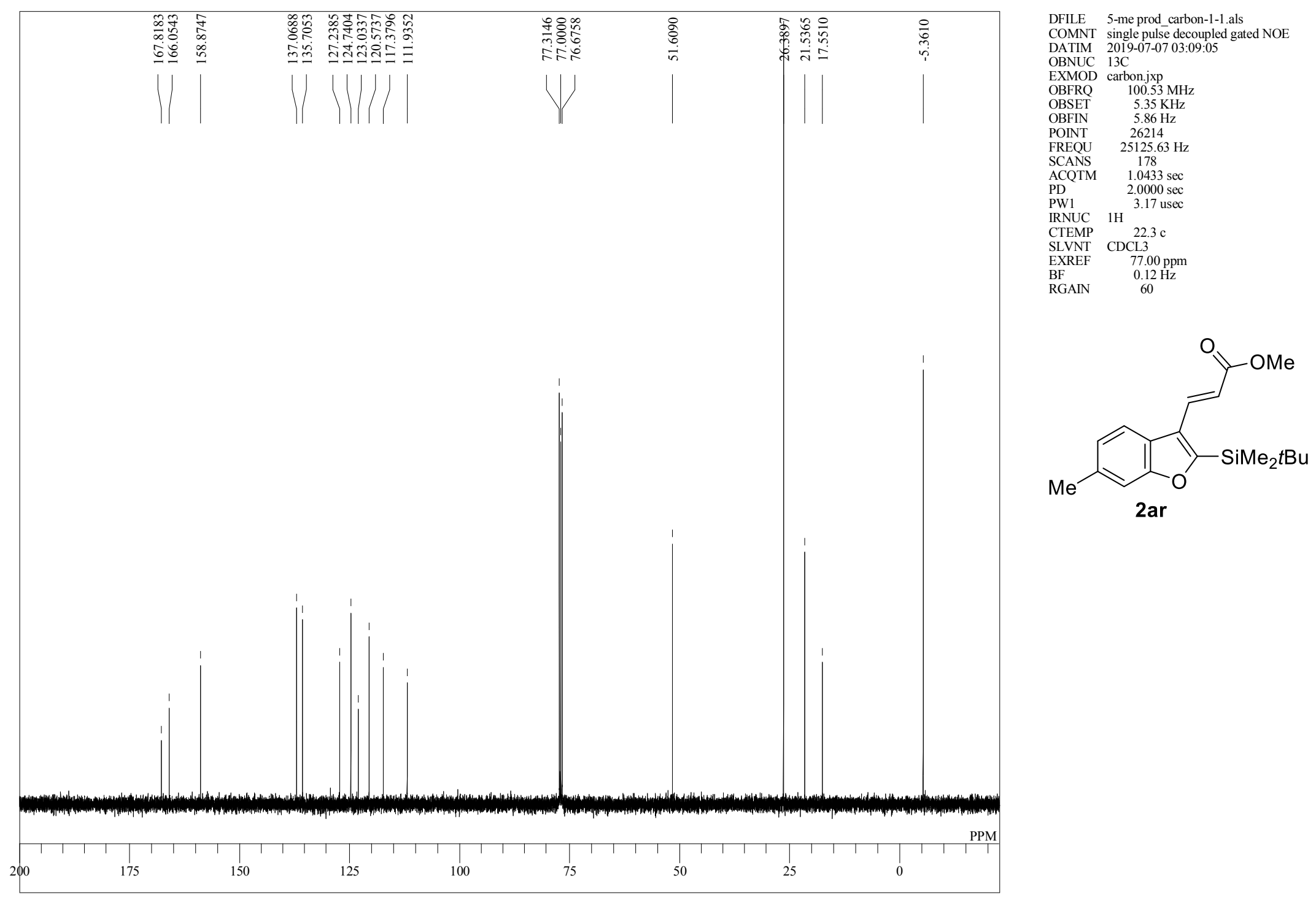

S2- 113 
single pulse

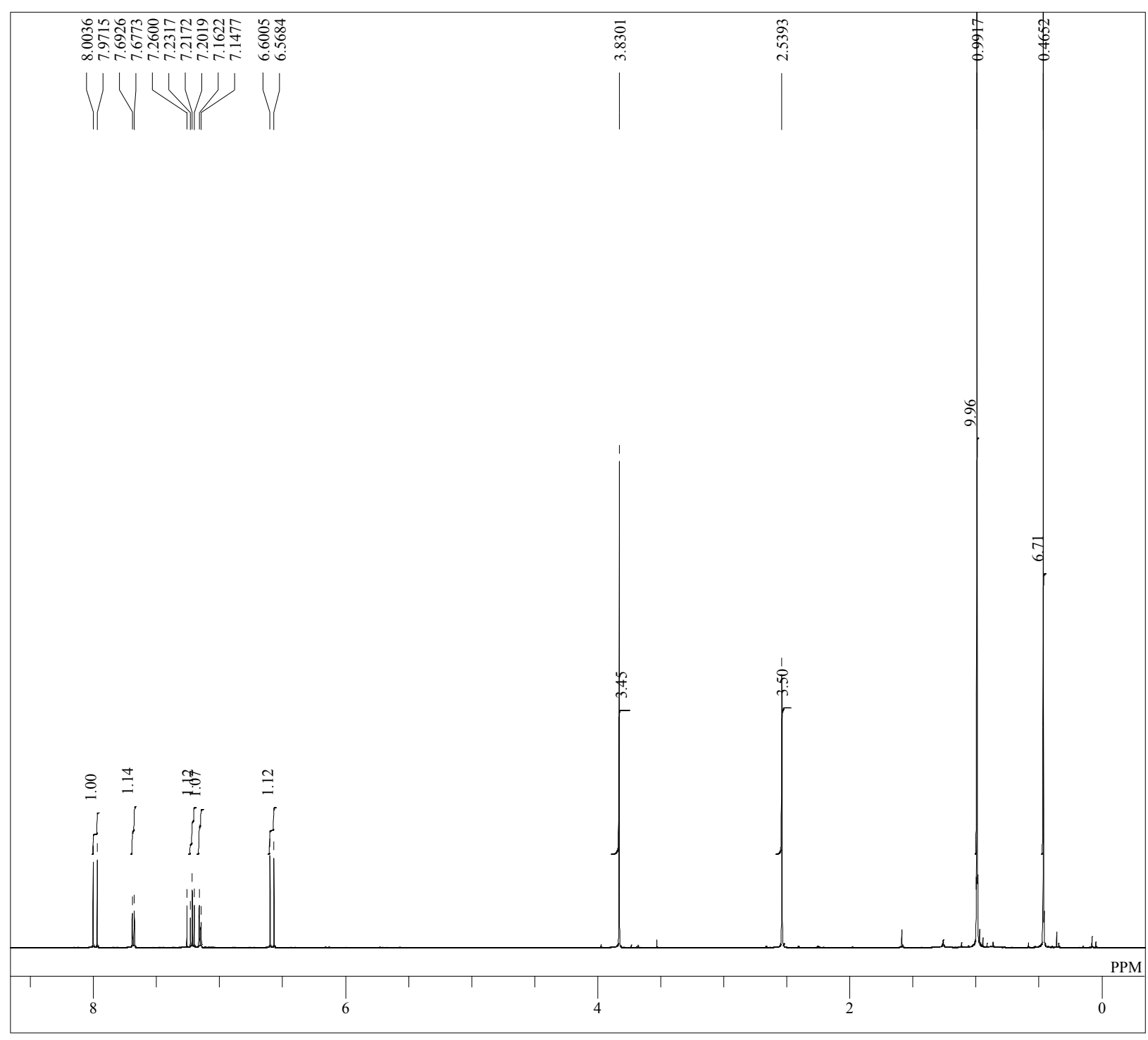

DFILE 6- Me prod_proton-1-1.als

COMNT single pulse

OBNUC $1 \mathrm{H}$

$\begin{array}{ll}\text { EXMOD } & \text { proton.jxp } \\ \text { OBFRQ } & 500.16 \mathrm{MHz}\end{array}$

$\begin{array}{ll}\text { OBSET } & 2.41 \mathrm{KHz} \\ \text { OBFIN } & 601 \mathrm{~Hz}\end{array}$

POINT 26214

FREQU $\quad 10020.04 \mathrm{~Hz}$

SCANS 16

$\begin{array}{ll}\text { ACQTM } & 2.6162 \mathrm{sec} \\ \mathrm{PD} & 2.0000 \mathrm{sec}\end{array}$

PW1 $\quad 6.50$ usec

IRNUC $1 \mathrm{H}$

$\begin{array}{ll}\text { CTEMP } & 22.7 \\ \text { SLVVNT } & \text { CDCL3 }\end{array}$

$\begin{array}{ll}\text { EXREF } & 7.26 \mathrm{ppm} \\ \mathrm{BF} & 0.12 \mathrm{~Hz}\end{array}$

RGAIN

$7.26 \mathrm{ppm}$
$0.12 \mathrm{~Hz}$
36

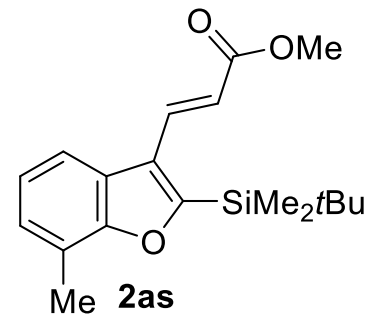

S2 - 114 
single pulse decoupled gated NOE

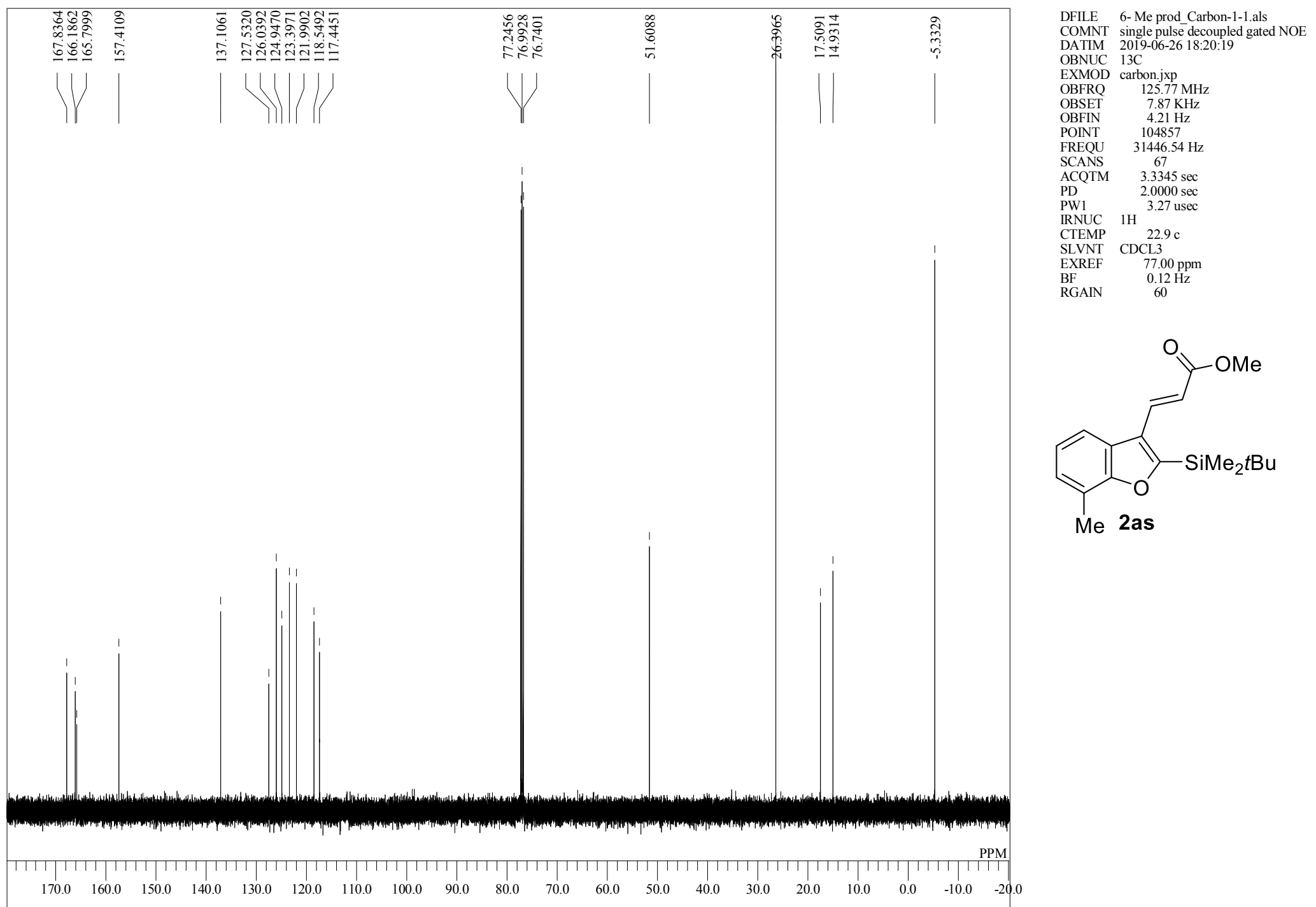


single pulse

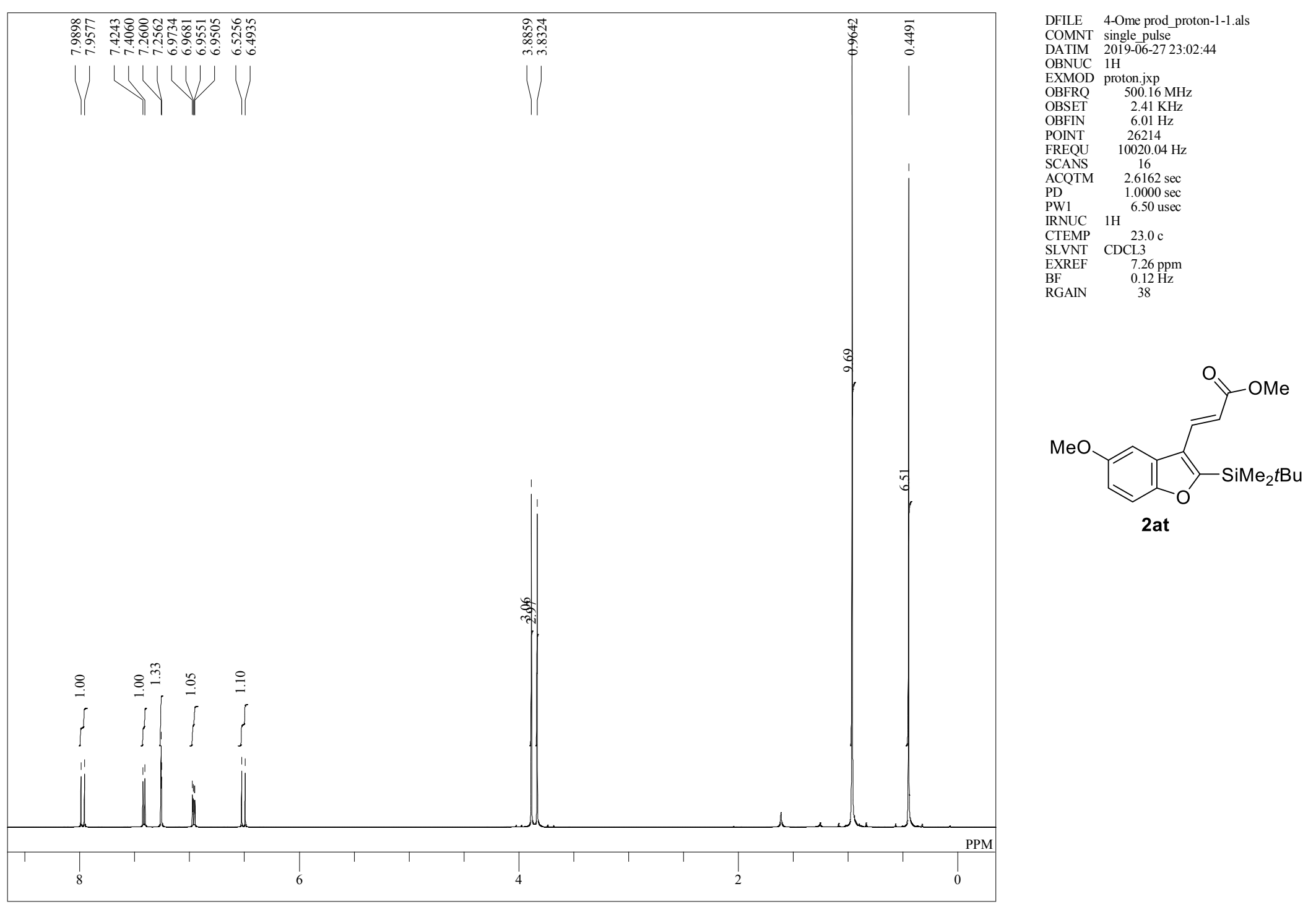

S2- 116 
single pulse decoupled gated NOE

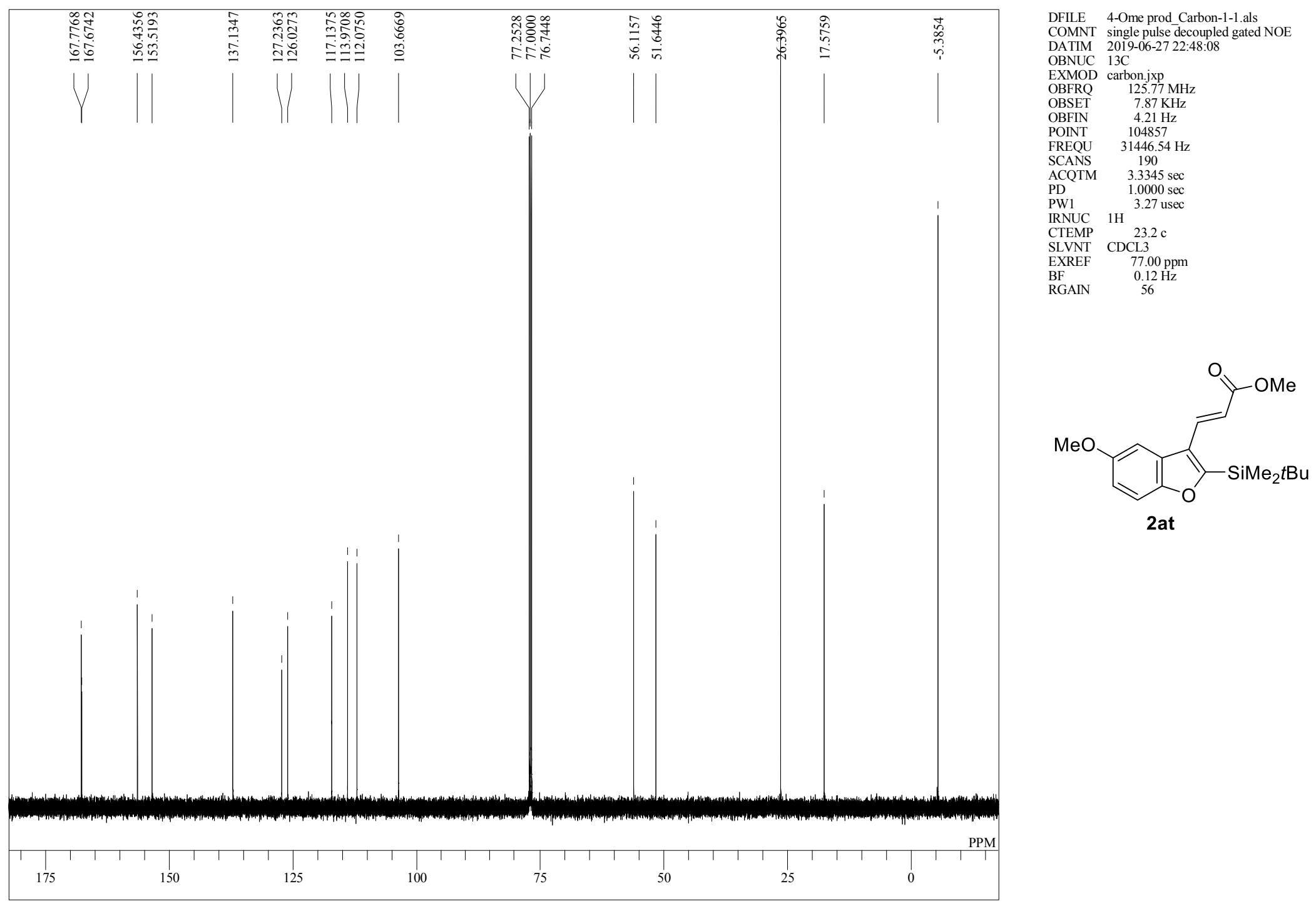

S2- 117 
single pulse

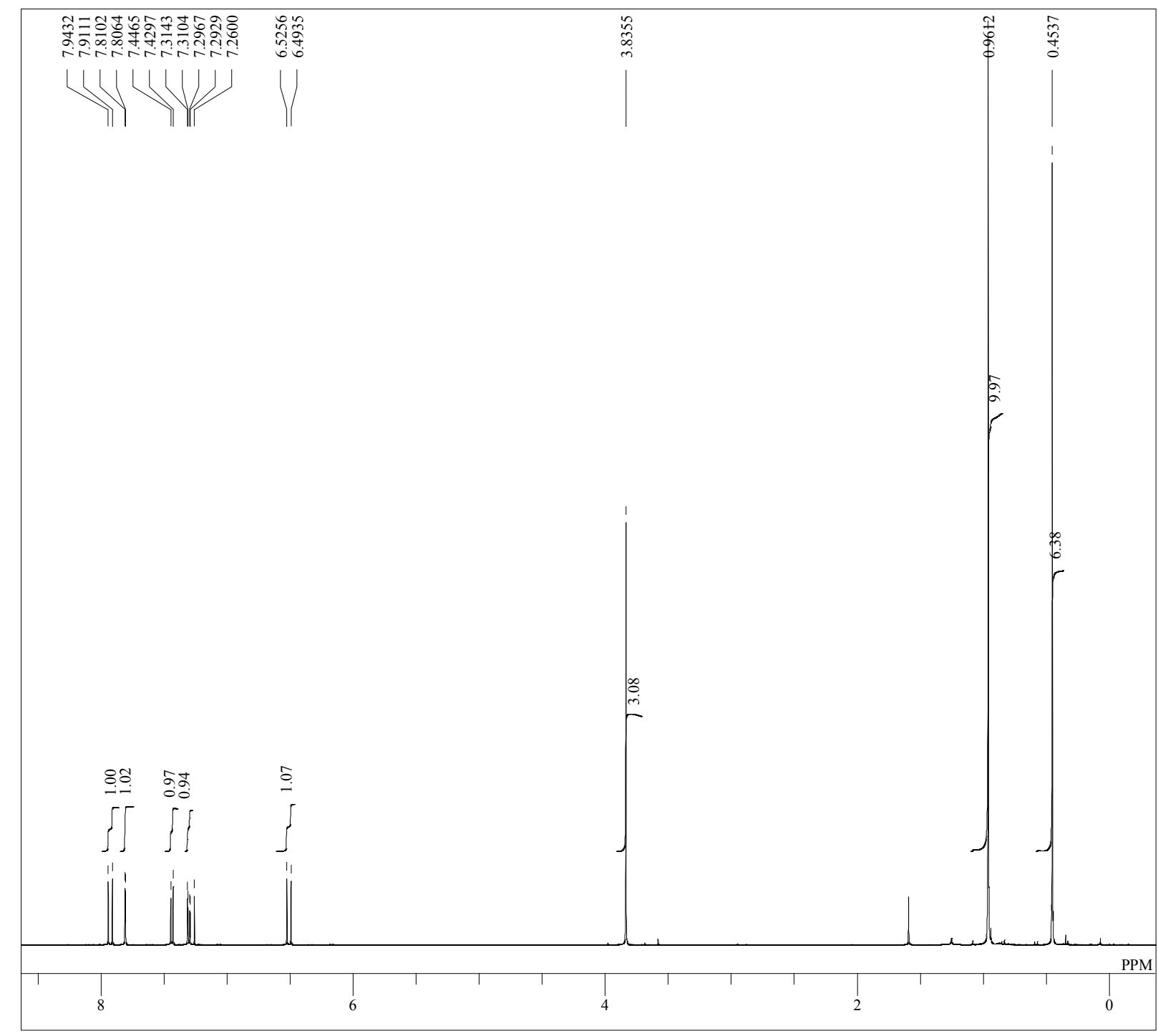

DFILE 4-cl prod proton-1-1.als

DATIM 2019-06-27 12:28:04

EXMOD proton

OBFRQ $50.16 \mathrm{MHz}$

OBSET $\quad 2.41 \mathrm{KHz}$

OBFIN $\quad 2.01 \mathrm{~Hz}$

FREQU $10020.04 \mathrm{~Hz}$

$\begin{array}{lll}\text { SCANS } & 16 \\ & & 16 \\ \text { ACQTM } & 26162 \mathrm{sec}\end{array}$

$\begin{array}{ll}\text { ACQTM } & 2.6162 \mathrm{sec} \\ \mathrm{PD} & 2.0000 \mathrm{sec}\end{array}$

PW1 $\quad 6.50 \mathrm{usec}$

IRNUC 1H

CTEMP 22.9

$\begin{array}{lc}\text { SLVNT } & \text { CDCL3 } 3 \\ \text { EXREF } & 7.26 \mathrm{ppm}\end{array}$

$\begin{array}{ll}\text { BF } & 0.12 \mathrm{~Hz} \\ \text { RGAIN } & 38\end{array}$

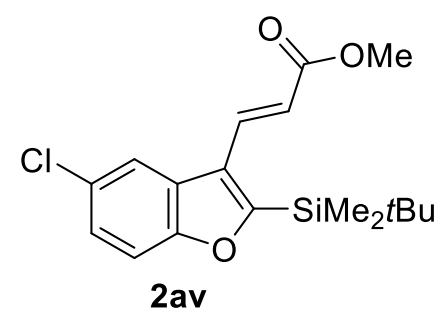

$\mathrm{S} 2-118$ 
single pulse decoupled gated NOE

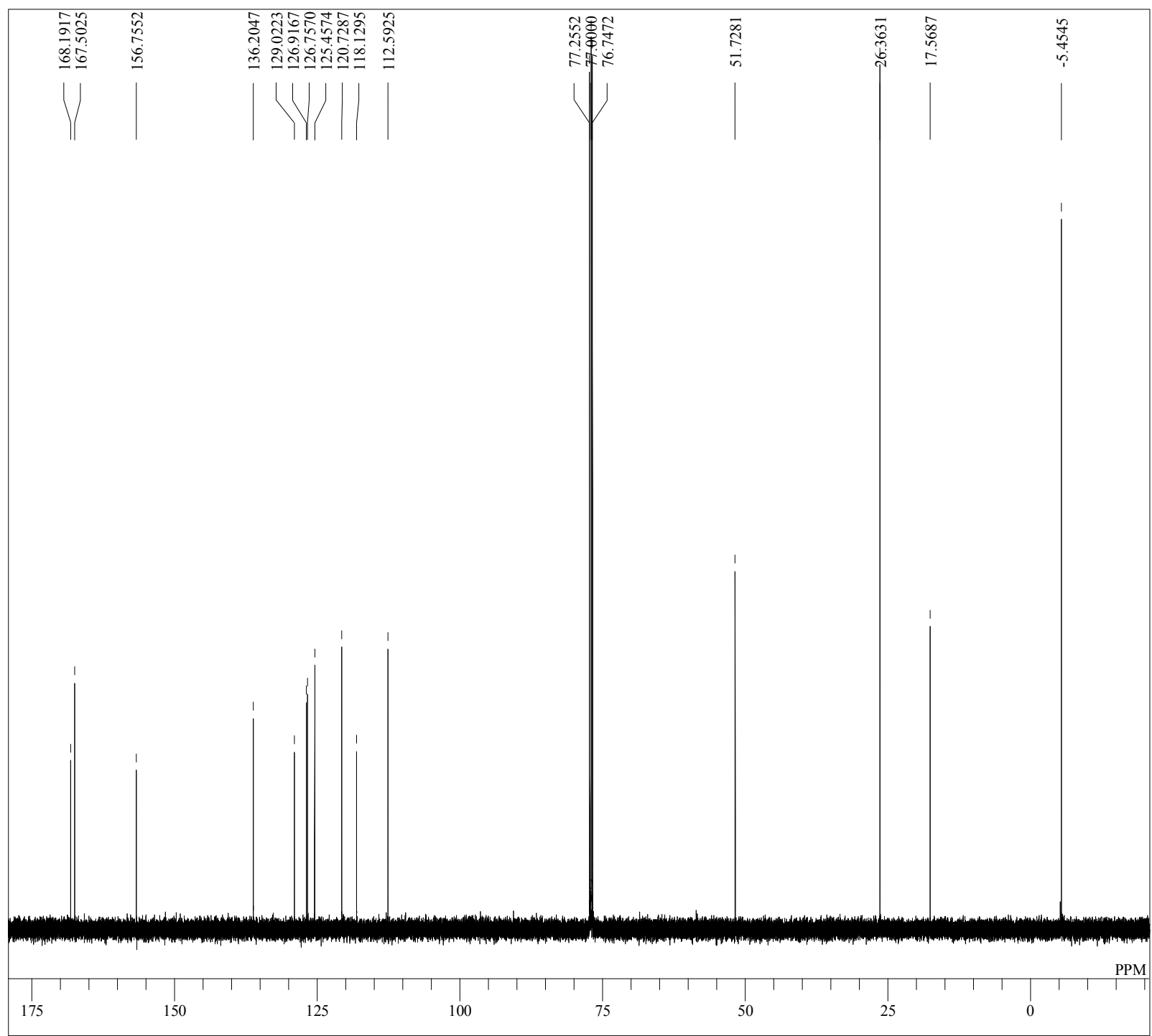

DFILE 4-cl prod Carbon-1-1.als

COMNT single pulse decoupled

B BNUC $13 \mathrm{C}$

EMMOD carbon.jxp

$\begin{array}{ll} & \\ \text { OBFRQ } & 125.77 \mathrm{MHz} \\ \text { OBSET } & 4.87 \mathrm{KHz}\end{array}$

$\begin{array}{ll}\text { OBFIN } & 4.21 \mathrm{~Hz} \\ \text { POINT } & 104857\end{array}$

FREQU $\quad 31446.54 \mathrm{~Hz}$

SCANS 174

$\begin{array}{ll}\text { ACQTM } & 3.3345 \mathrm{sec} \\ \mathrm{PD} & 2.0000 \mathrm{sec}\end{array}$

$\begin{array}{ll}\text { PD } & 2.0000 \mathrm{sec} \\ \text { PW1 } & 3.27 \text { usec }\end{array}$

$\begin{array}{lll}\text { IRNUC } & 1 \mathrm{H} & \\ \text { CTEMP } & & 23.2\end{array}$

SLVNT CDCL3

$\begin{array}{ll}\mathrm{BF} & 0.12 \mathrm{~Hz}\end{array}$

$77.00 \mathrm{ppm}$
$0.12 \mathrm{~Hz}$
60

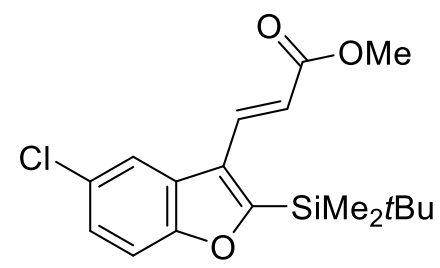

2av 
single $\_$pulse

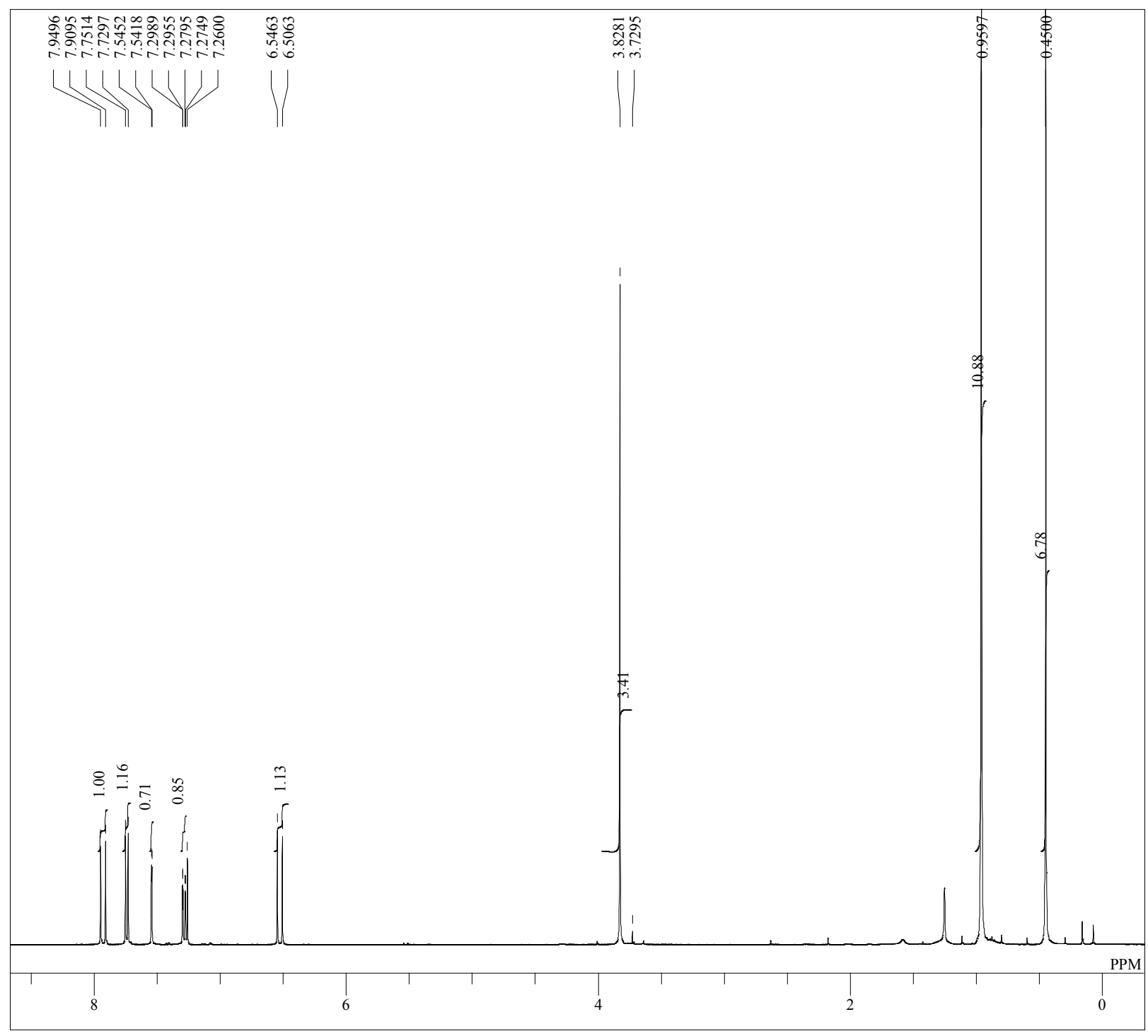

DFILE 5-cl prod proton-1-1.als

$\begin{array}{ll}\text { COMNT } & \text { single pulse } \\ \text { DATIM } & 2019-07-0620: 20: 41\end{array}$

OBNUC $1 \mathrm{H}$

EXMOD

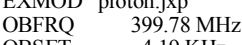

$\begin{array}{ll}\text { OBSET } & 4.19 \mathrm{KHz} \\ \text { OBFIN } & 7.29 \mathrm{~Hz}\end{array}$

POINT $\quad 13107$

FREQU $\quad 6002.40 \mathrm{~Hz}$

$\begin{array}{ll}\text { SCANS } & 8 \\ \text { ACOTM } & 2.1837 \mathrm{sec}\end{array}$

$\begin{array}{lll}\mathrm{PD} & 2.1837 \mathrm{sec} \\ & 1.0000 \mathrm{sec}\end{array}$

\begin{tabular}{ll} 
PW1 & $1.0000 \mathrm{sec}$ \\
\hline RWWC & $7.25 \mathrm{usec}$
\end{tabular}

IRNUC $1 \mathrm{H}$

$\begin{array}{ll}\text { CTEMP } & 22.0 \mathrm{c} \\ \text { SLVNT } & \text { CDCL3 }\end{array}$

$\begin{array}{ll}\text { EXREF } & 7.26 \mathrm{ppm} \\ \mathrm{BF} & 0.12 \mathrm{~Hz}\end{array}$

RGAIN 40

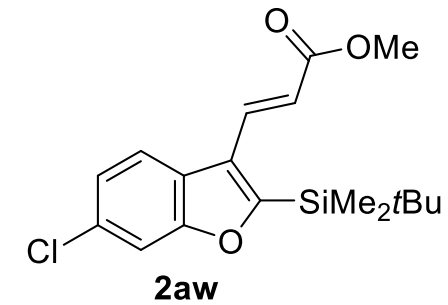

S2 - 120 
single pulse decoupled gated NOE

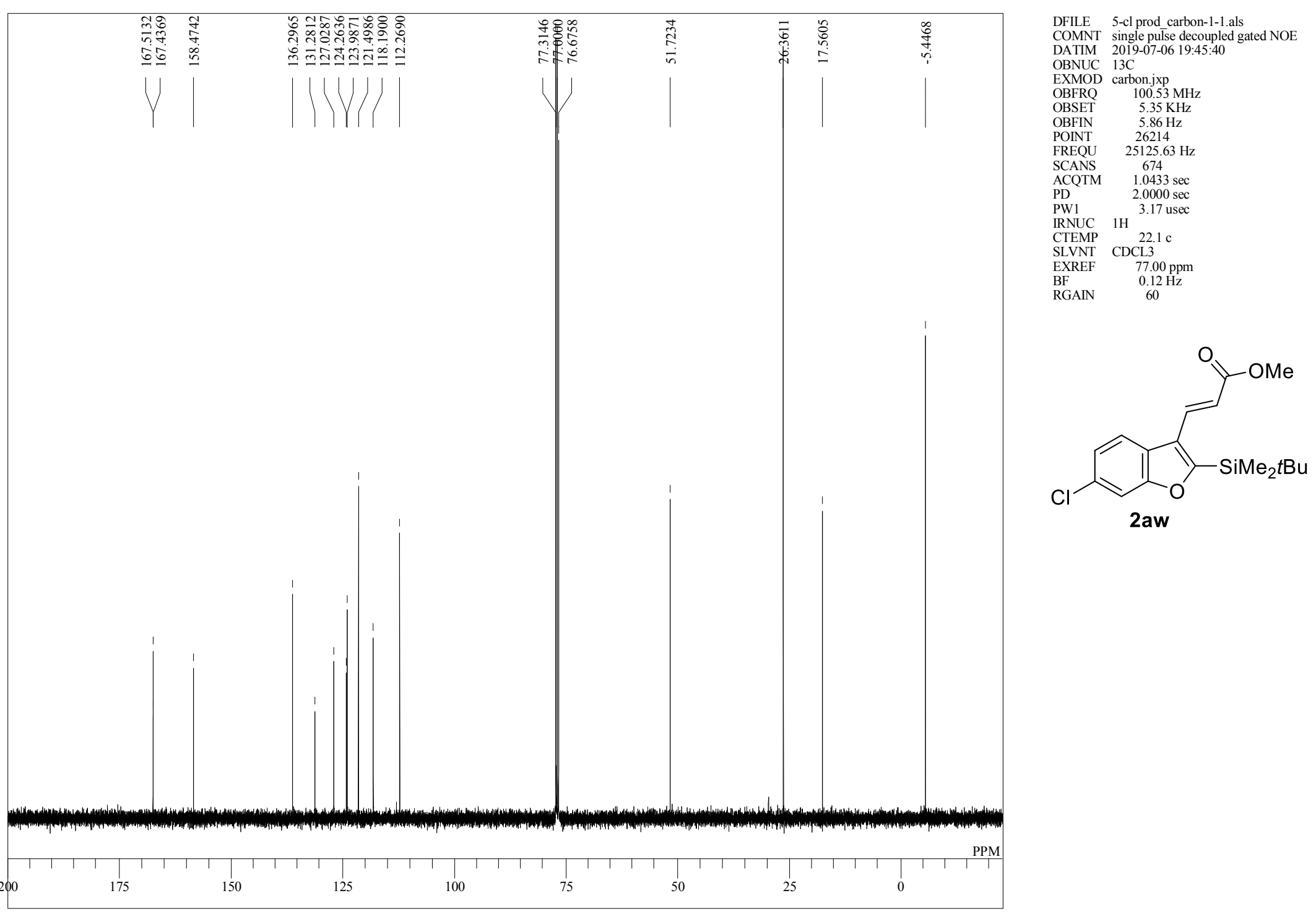

S2 - 121 
single pulse

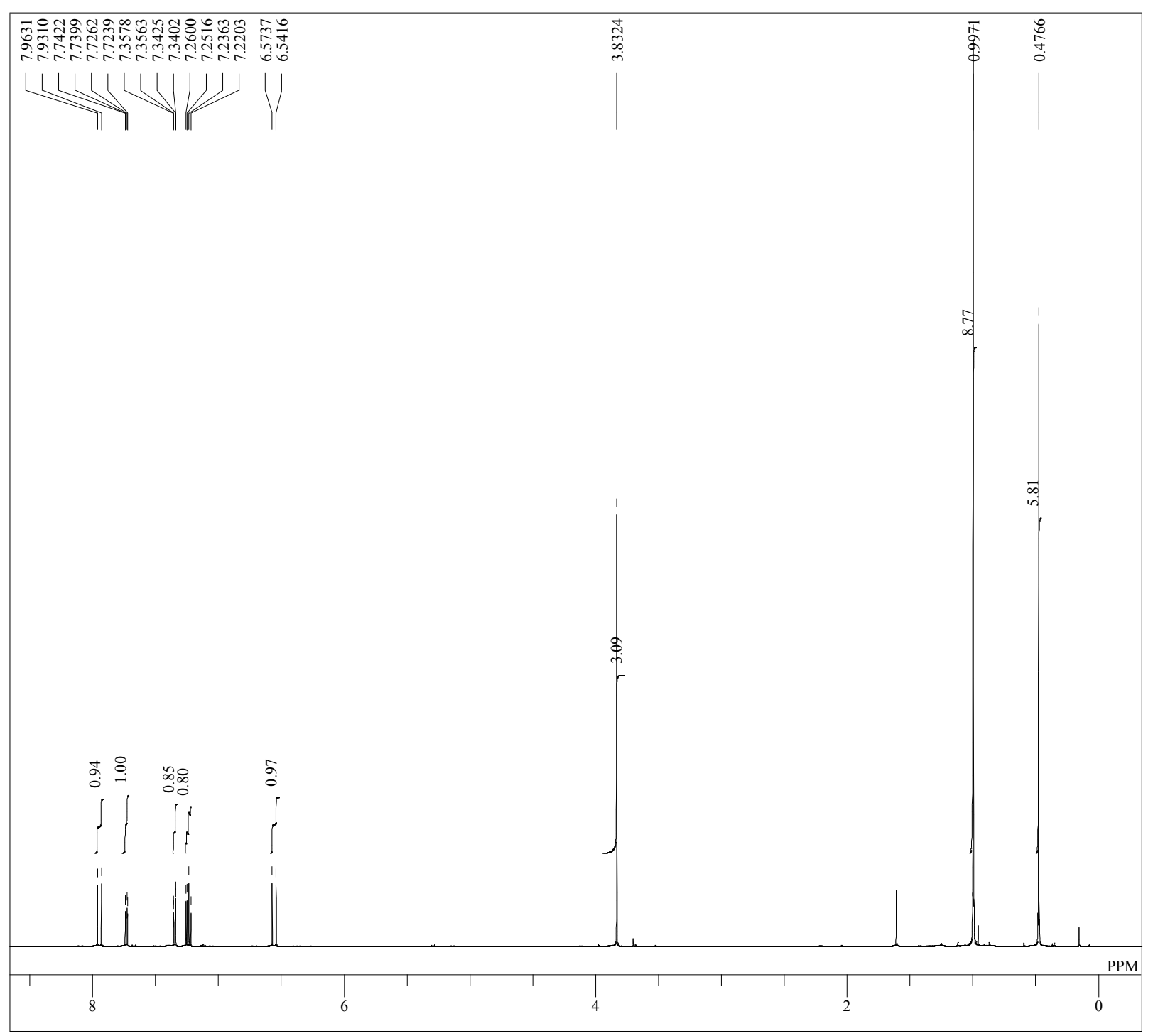

DFILE 6-cl prod_proton-1-1.als

COMNT single pulse

OBNUC

EXMOD proton.jxp
OBFRO $500.16 \mathrm{MH}$

OBSET $\quad 2.41 \mathrm{KHz}$

$\begin{array}{ll}\text { POINT } & 6.01 \mathrm{~Hz} \\ & 26214\end{array}$

FREQU $\quad 10020.04 \mathrm{H} z$

$\begin{array}{ll}\text { SCANS } & 16 \\ \text { ACQTM } & 2.6162 \mathrm{sec} \\ \text { PD } & 20000 \mathrm{sec}\end{array}$

PW1 $\quad 6.50 \mathrm{usec}$

IRNUC 1H

CTEMP $22.6 \mathrm{c}$

$\begin{array}{ll}\text { EXREF } & 7.26 \mathrm{ppm} \\ \mathrm{BF} & 0.12 \mathrm{H} Z\end{array}$

$\begin{array}{ll}\text { BF } & 0.12 \mathrm{~Hz} \\ \text { RGAIN } & 36\end{array}$

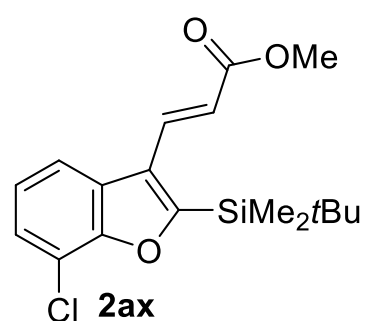

Cl 2ax 
single pulse decoupled gated NOE

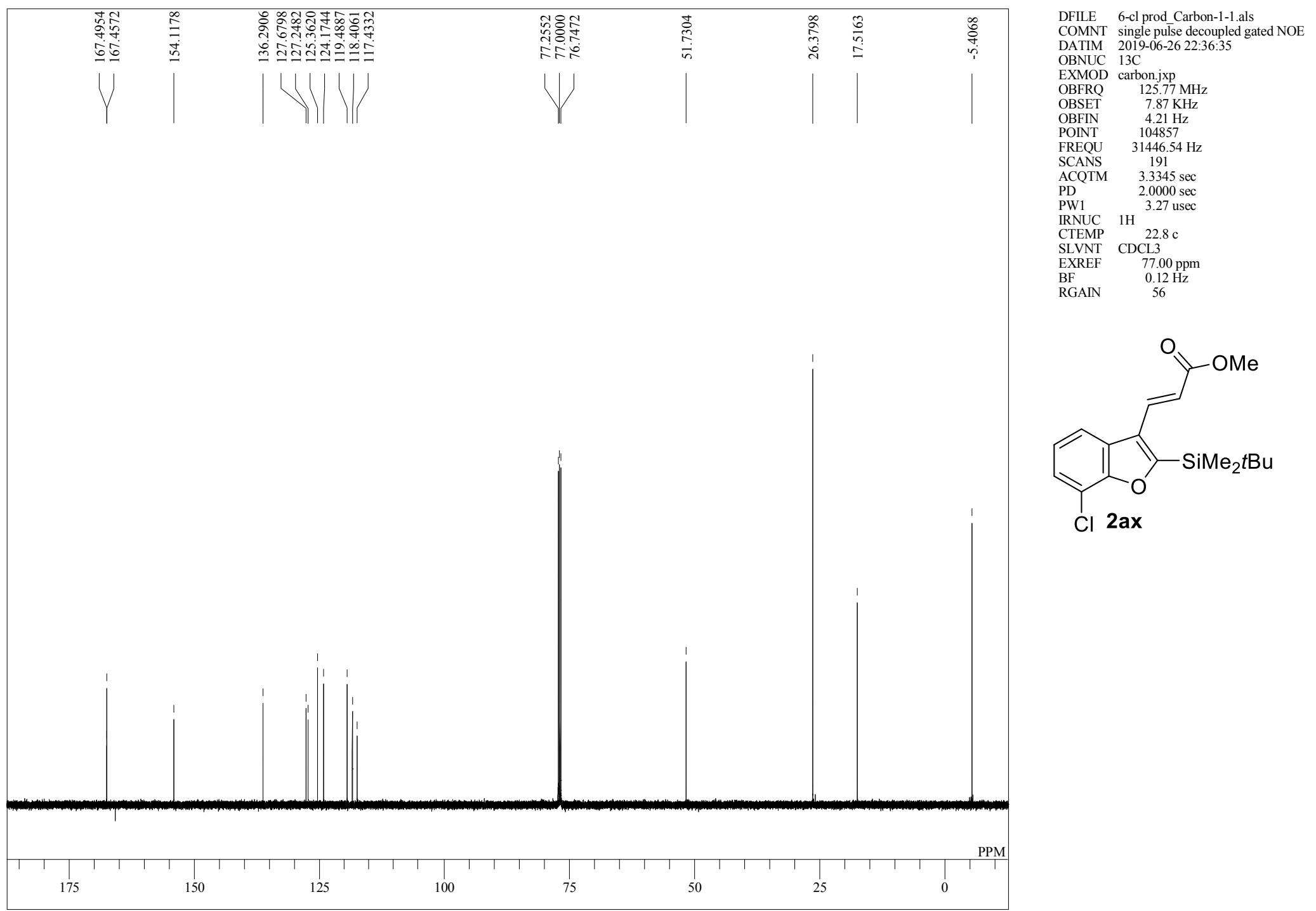

S2- 123 
single pulse

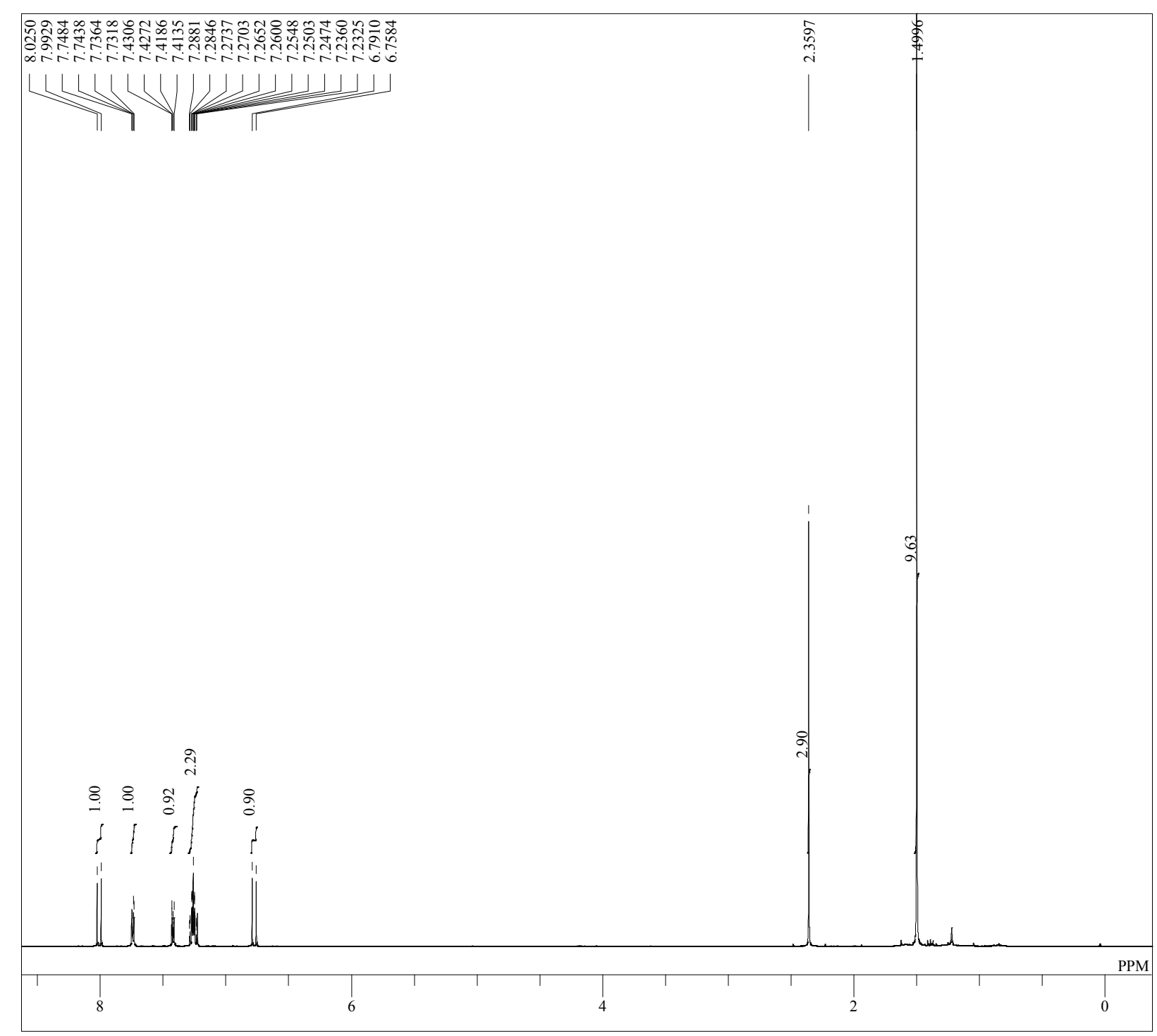

DFILE calculate product_proton-1-1.als

$\begin{array}{ll}\text { COMNT } & \text { single pulse } \\ \text { DATIM } & \text { 2019-08-23 04:12:19 }\end{array}$

OBNUC $1 \mathrm{H}$

$\begin{array}{ll}\text { EXMOD } & \text { proton.jxp } \\ \text { OBFRQ } & 500.16 \mathrm{MHz}\end{array}$

OBSET $\quad 2.41 \mathrm{KHz}$

POINT 26214

FREQU $\quad 7507.51 \mathrm{~Hz}$

\begin{tabular}{lll} 
SCANS & 16 \\
\hline ACQTM & 3.4918
\end{tabular}

$\mathrm{PD} \quad-\quad 3.4918 \mathrm{sec}$

PW1 6.50 usec

IRNUC $1 \mathrm{H} \quad 23.4 \mathrm{c}$

$\begin{array}{ll}\text { CTEMP } & 23.4 \\ \text { SLVNT } & \text { CDCL } 3\end{array}$

$\begin{array}{ll}\text { EXREF } & 7.26 \mathrm{ppm} \\ \mathrm{BF} & 0.12 \mathrm{~Hz}\end{array}$

RGAIN $\quad 40 \mathrm{~Hz}$

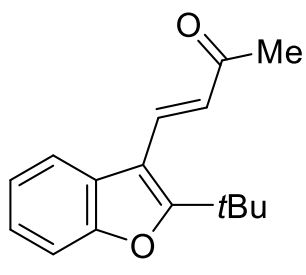

2ay

$\mathrm{S} 2-124$ 
single pulse decoupled gated NOE

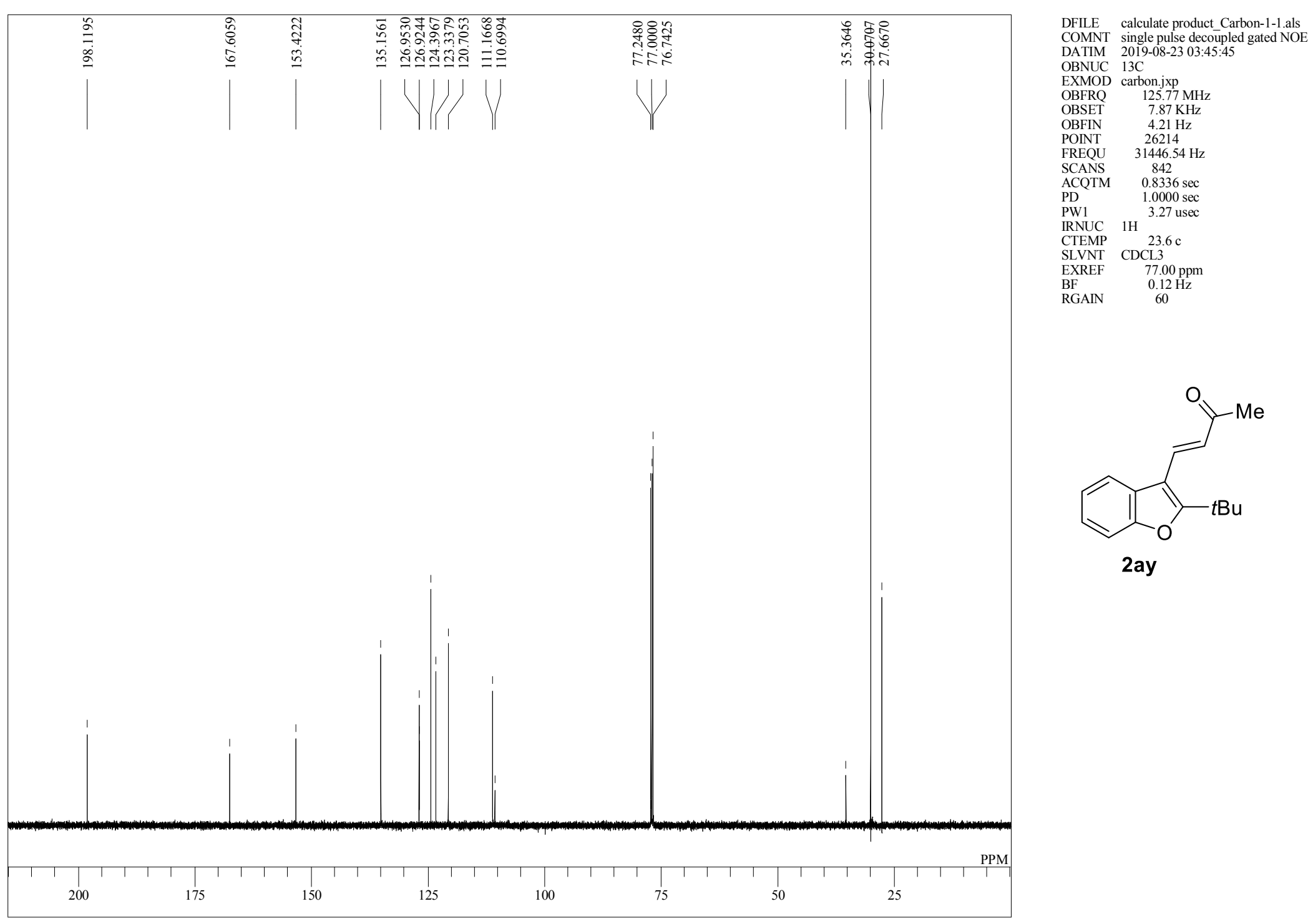

S2- 125 
single pulse

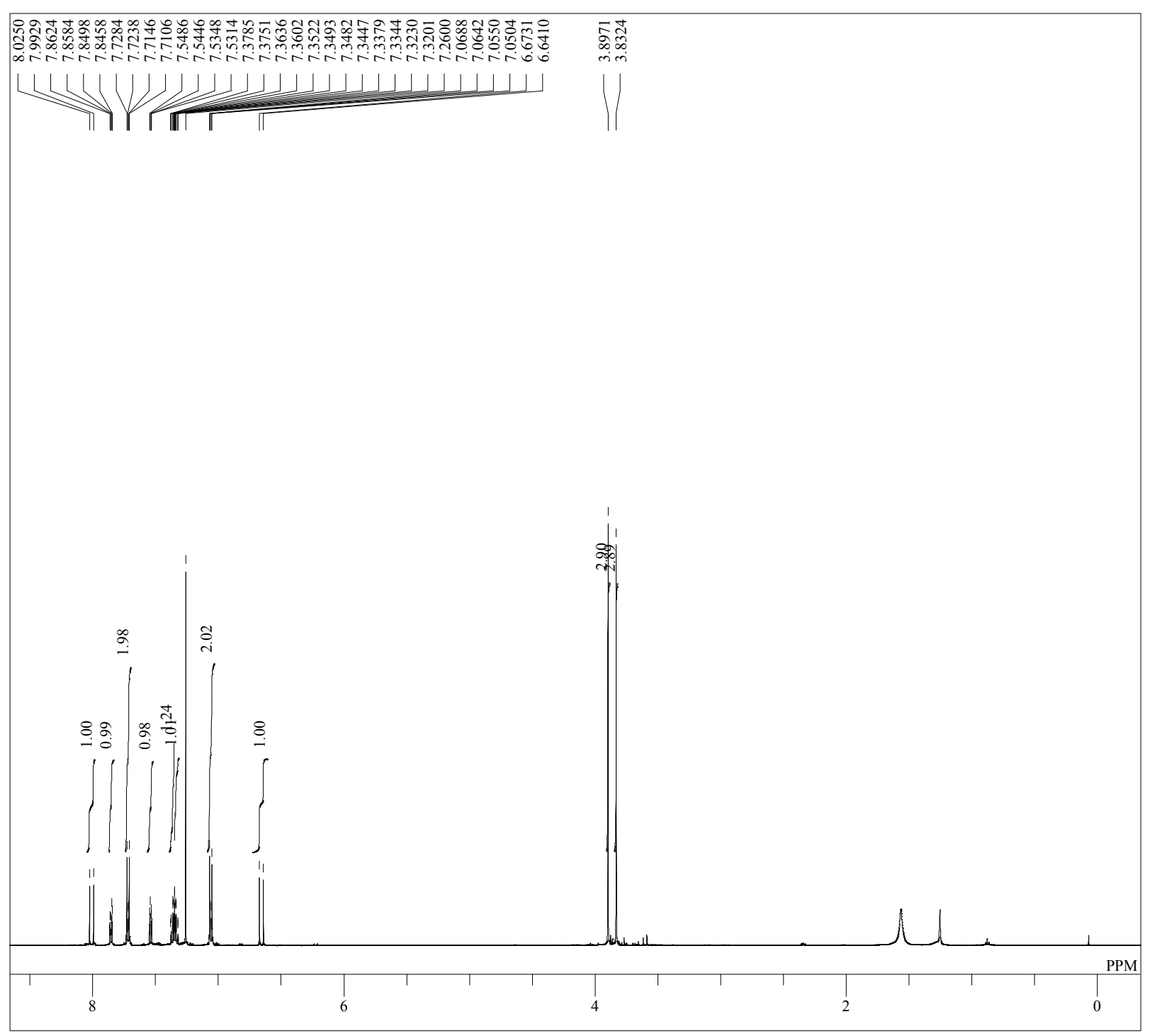

DFILE prod hiyama_proton-1-1.als

$\begin{array}{ll}\text { COMNT single pulse } & \\ \text { DATIM } & 2019-08-01 ~ 01: 30: 46\end{array}$

OBNUC $1 \mathrm{H}$

EXMOD proton.jxp

OBSET $\quad 2.41 \mathrm{KHz}$

$\begin{array}{ll}\text { OBFIN } & 6.01 \mathrm{~Hz} \\ \text { POINT } & 26214\end{array}$

FREQU $\quad 7507.51 \mathrm{~Hz}$

SCANS 16

$\begin{array}{ll}\text { ACQTM } & 3.4918 \mathrm{sec} \\ \mathrm{PD} & 6.000 \mathrm{scc}\end{array}$

$\begin{array}{ll}\text { PW1 } & 2.0000 \mathrm{sec} \\ \text { PW1 } & 6.50 \mathrm{usec}\end{array}$

$\begin{array}{ll}\text { IRNUC } & 1 \mathrm{H} \\ \text { CTEMP } & 24.2 \mathrm{c}\end{array}$

SLVNT CDCL3 $24.2 \mathrm{c}$

EXREF $7.26 \mathrm{ppm}$

$\begin{array}{ll}\text { BF } & 0.12 \mathrm{~Hz} \\ \text { RGAIN } & 54\end{array}$

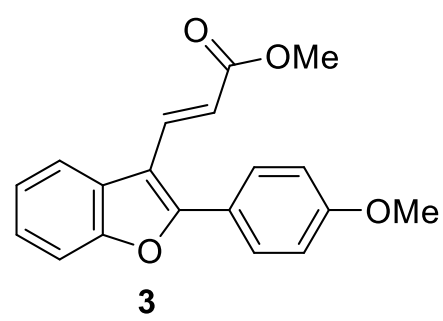

$\mathrm{S} 2-126$ 
single pulse decoupled gated NOE

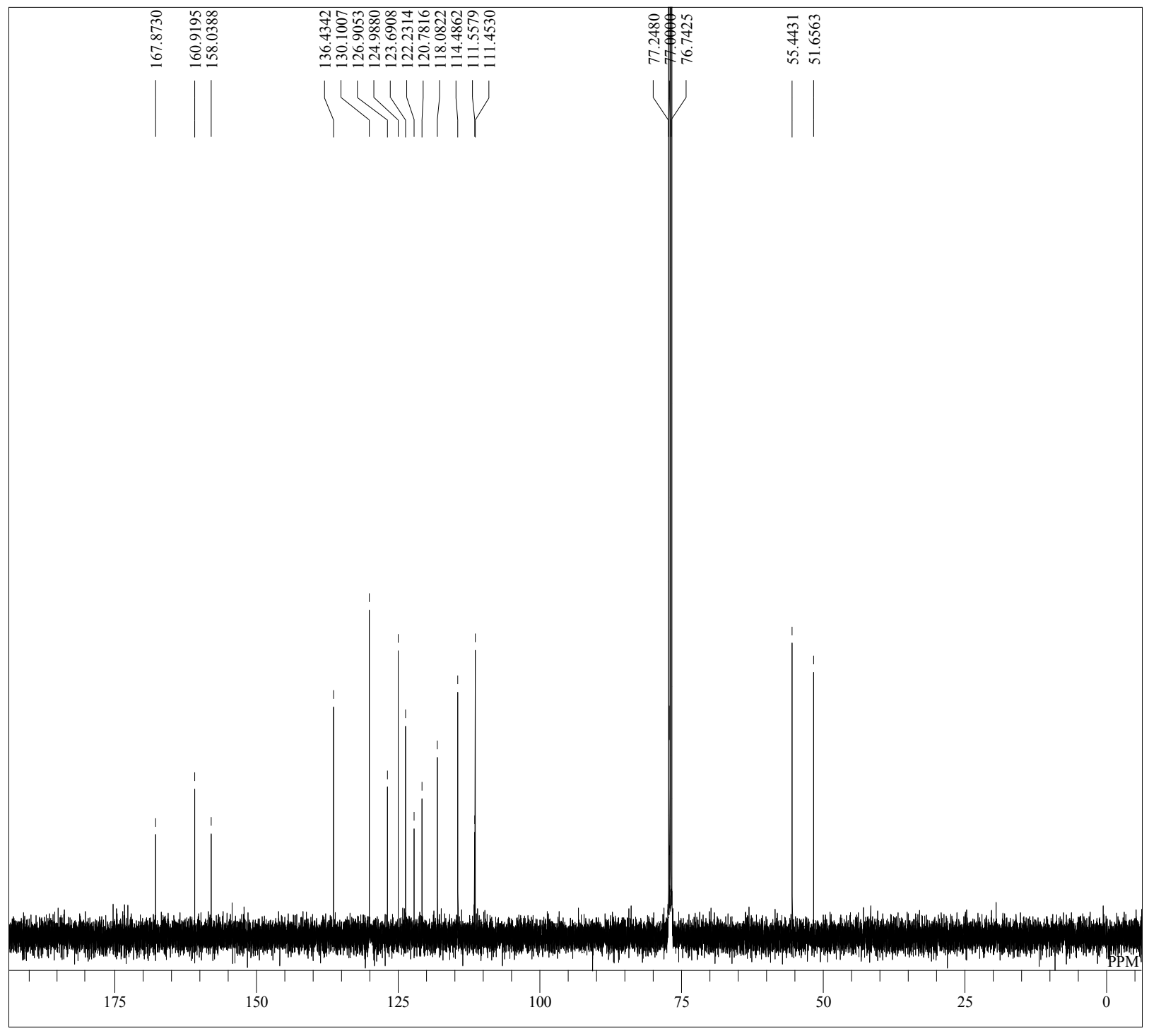

DFILE prod hiyama Carbon-1-1.als

COMNT single pulse decoupled gated NOE

OBNUC 13C

EXMOD carb

OBFRQ $\quad 125.77 \mathrm{MHz}$

$\begin{array}{ll}\text { OBSET } & 7.87 \mathrm{KHz} \\ \text { OBFIN } & 4.21 \mathrm{~Hz}\end{array}$

$\begin{array}{ll}\text { POINT } & 26214\end{array}$

FREQU $\quad 31446.54 \mathrm{~Hz}$

SCANS $\quad 3039$

$\begin{array}{ll}\text { ACQTM } & 0.8336 \mathrm{sec} \\ \mathrm{PD} & 1.0000 \mathrm{sec}\end{array}$

PW1 $\quad 3.27$ usec

$\begin{array}{lll}\text { IRNUC } & 1 \mathrm{H} & \\ \text { CTEMP } & 24.5 \mathrm{c}\end{array}$

$\begin{array}{ll}\text { SLVNT } & \text { CDCL3 } \\ \text { EXREF } & 77.00 \mathrm{pp}\end{array}$

$\begin{array}{lc}\mathrm{BF} & 0.12 \mathrm{~Hz} \\ \mathrm{RGAIN} & 60\end{array}$

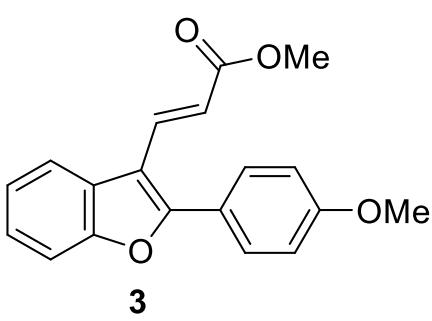

S2 - 127 
single pulse

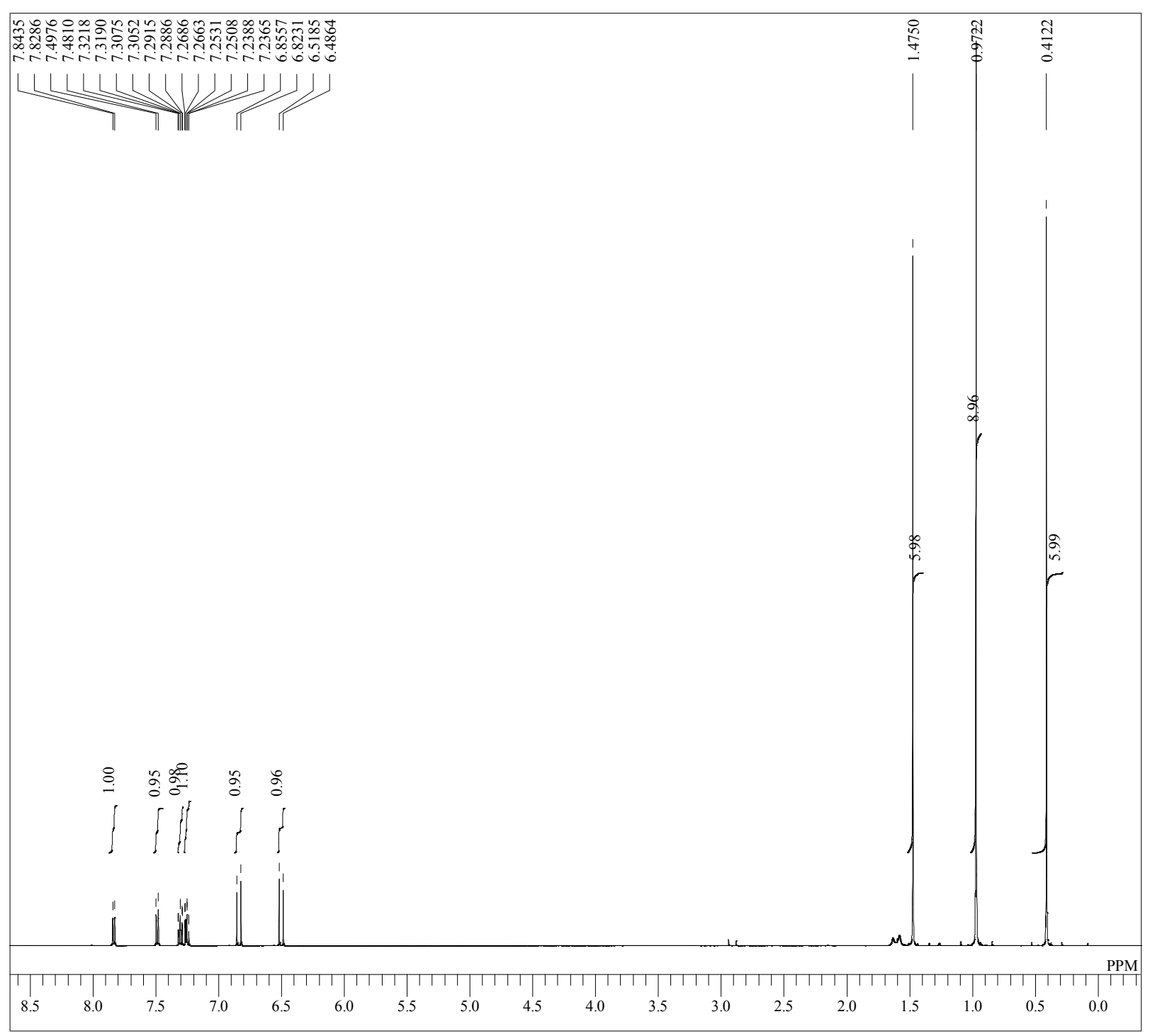

DFILE memgbr-reaction proton-1-1.als

$\begin{array}{ll}\text { COMNT } & \text { single pulse } \\ \text { DATIM } & 2019-08-21 \text { 14:21:19 }\end{array}$

OBNUC $1 \mathrm{H}$

EXMOD proton.jxp

$\begin{array}{ll}\text { OBFRQ } & 500.16 \mathrm{MHz} \\ \text { OBSET } & 2.41 \mathrm{KHz}\end{array}$

OBFIN $\quad 6.01 \mathrm{~Hz}$

$\begin{array}{ll}\text { POINT } & 26214 \\ \text { FREOU } & 7507.51 \mathrm{~Hz}\end{array}$

SCANS 16

PD $\quad 3.4918 \mathrm{sec}$

$\begin{array}{ll}\text { PD } & 2.0000 \mathrm{sec} \\ \text { PW1 } & 6.50 \mathrm{usec}\end{array}$

IRNUC $1 \mathrm{H}$

CTEMP $25.2 \mathrm{c}$

$\begin{array}{ll}\text { EXREF } & 7.26 \mathrm{ppm} \\ \mathrm{BF} & 0.12 \mathrm{~Hz}\end{array}$

RGAIN $\quad 32$

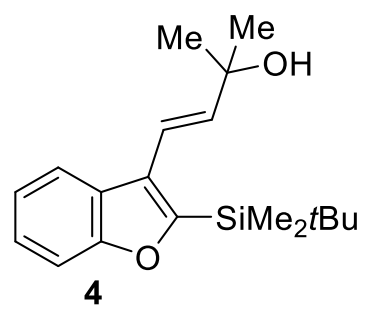

$\mathrm{S} 2-128$ 
single pulse decoupled gated NOE

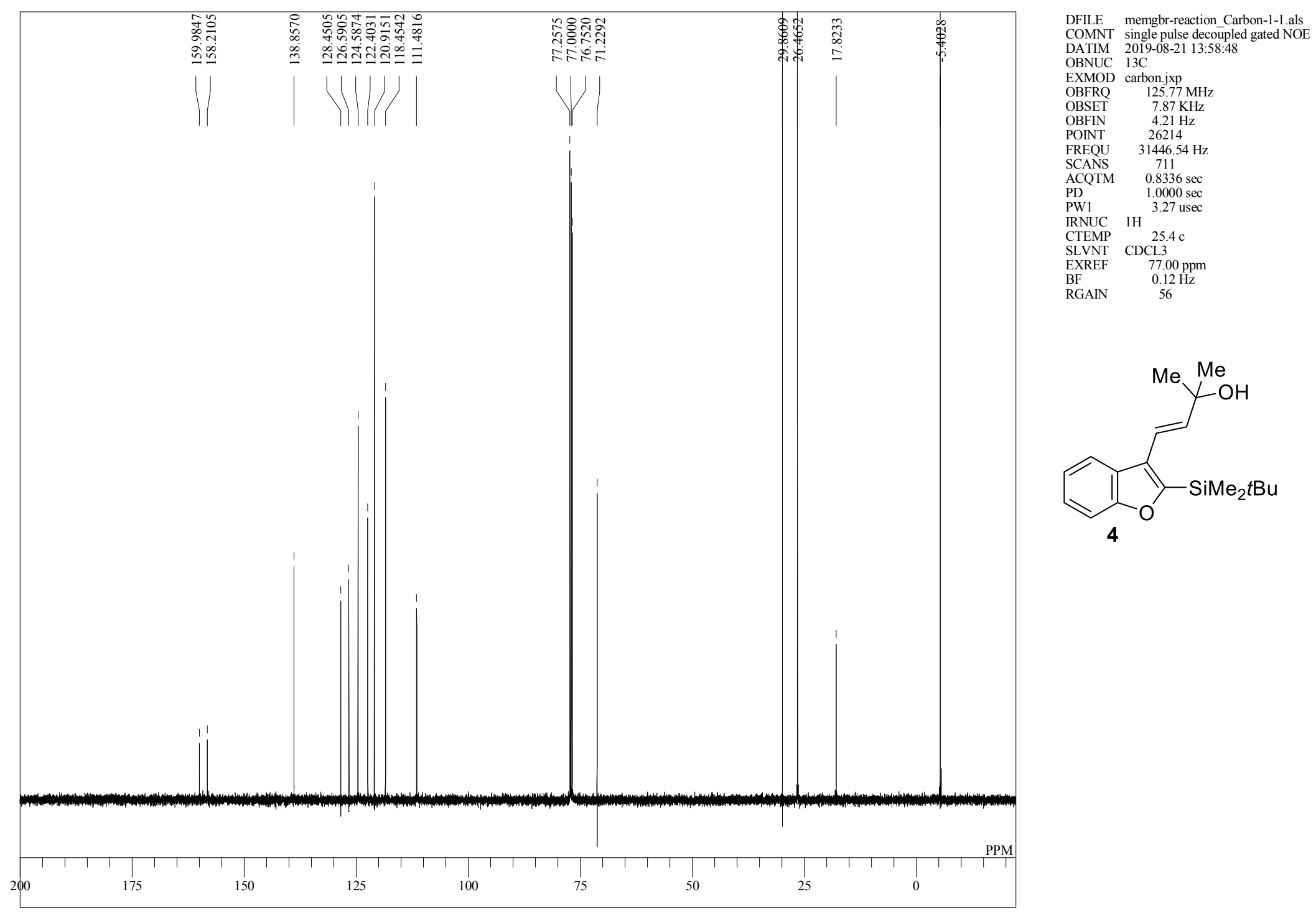

S2- 129 
single pulse

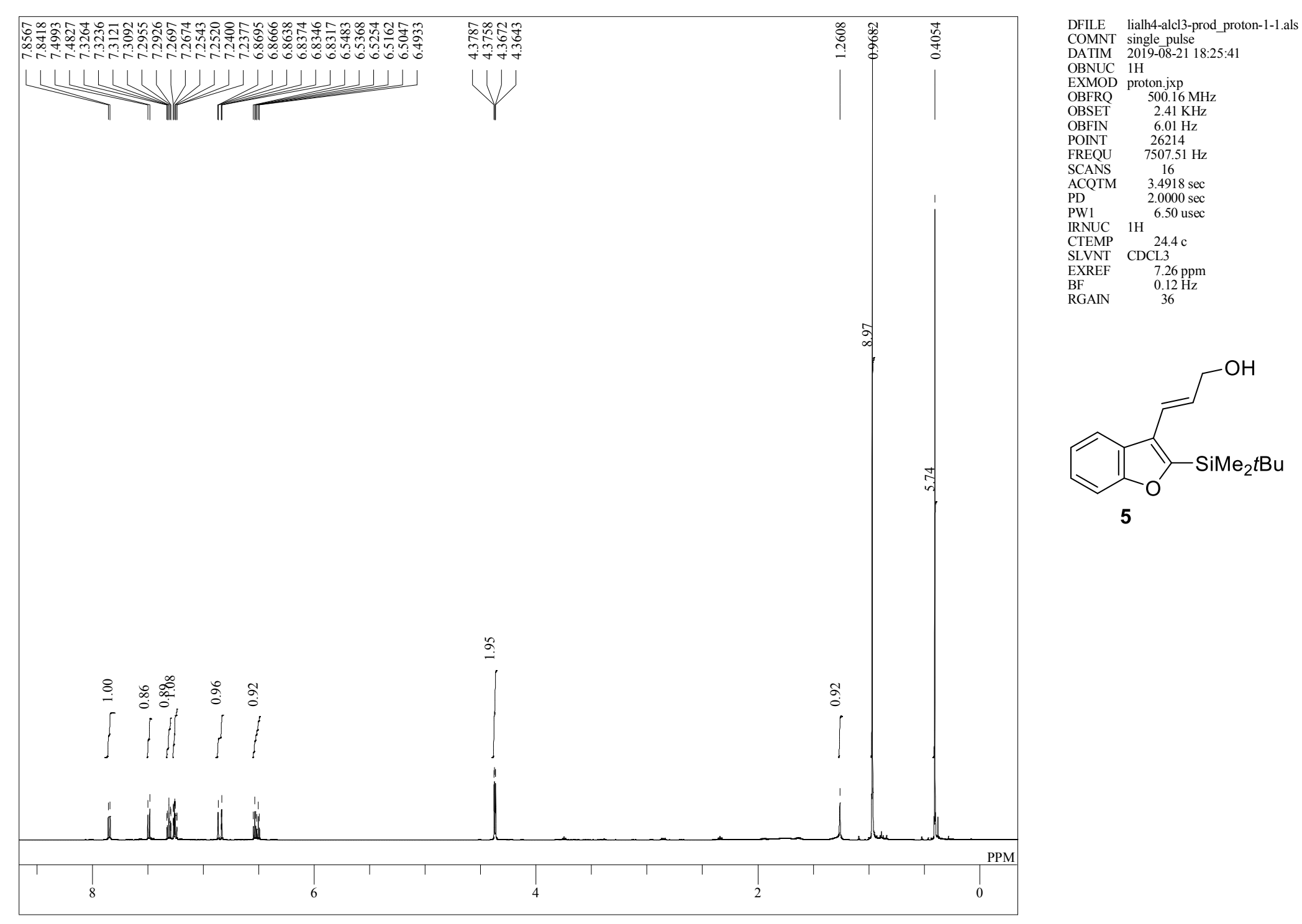

S2 - 130 
single pulse decoupled gated NOE

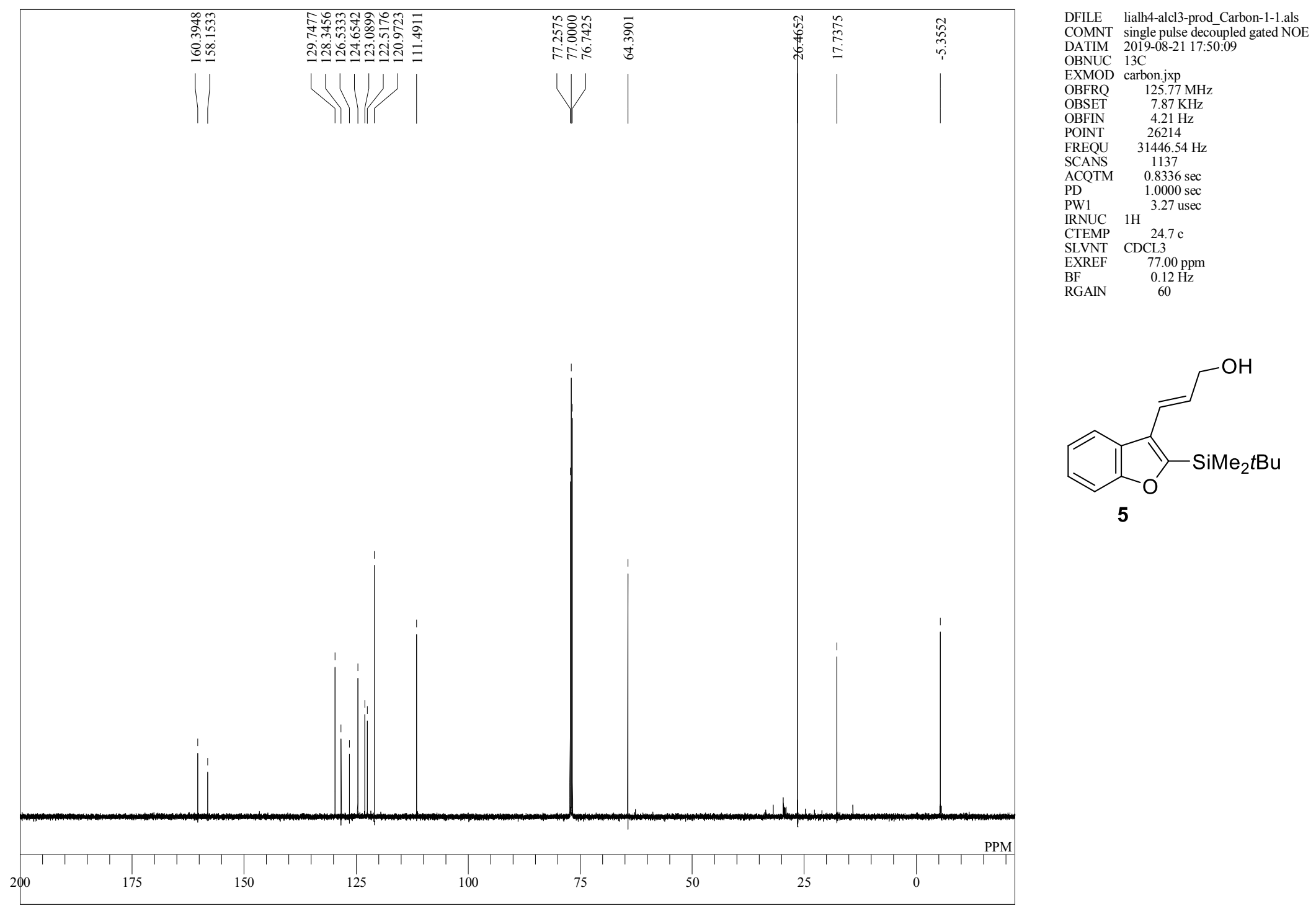

S2- 131 